\title{
Twofold $\pi$-Extension of Polyarenes via Double and Triple Radical Alkyne peri-Annulations: Radical Cascades Converging on the Same Aromatic Core
}

Edgar Gonzalez-Rodriguez, Miguel A. Abdo, Gabriel dos Passos Gomes, Suliman Ayad, Frankie D. White, Nikolay P. Tsvetkov, Kenneth Hanson and Igor V. Alabugin* 


\section{TABLE OF CONTENTS}

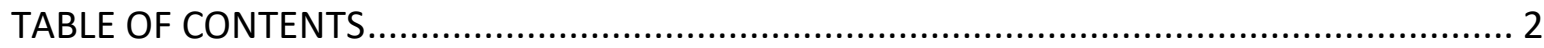

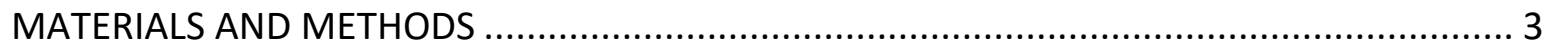

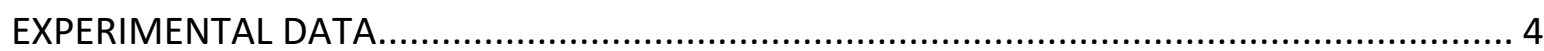

Synthesis of dicarbaldehyde starting materials for the preparation of radical precursors $1 \mathbf{a}-\mathbf{j} . . . . .4$

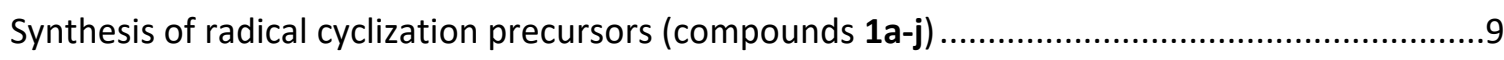

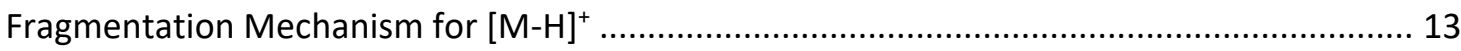

Double alkyne radical peri-annulations (compounds 2a-j) ..................................................... 13

Synthesis of (3,6-diphenylbenzo[e]pyrene-2,7-diyl)bis(tributylstannane) and (3,9diphenylperylene-2,8-diyl)bis(tributylstannane) (compounds $\mathbf{2 f}$ and $\mathbf{2 f} \mathbf{f}^{\prime}$ ) ............................. 17

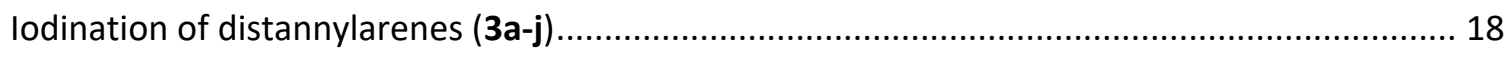

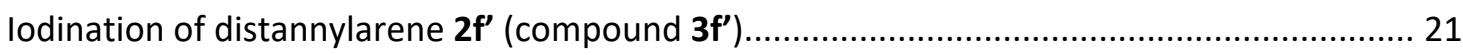

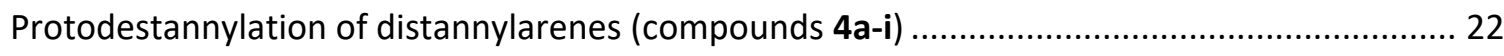

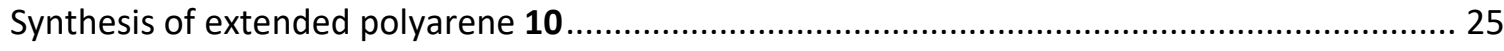

Optimization conditions for the Sn-promoted double peri-annulation ..................................... 27

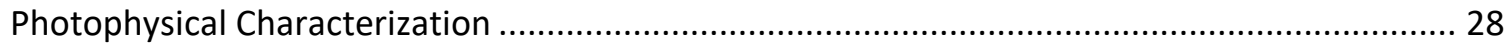

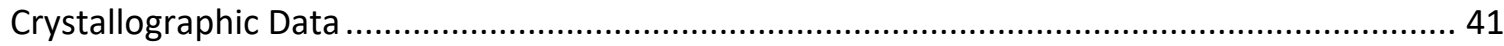

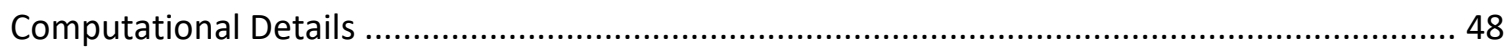

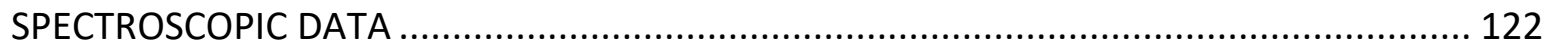

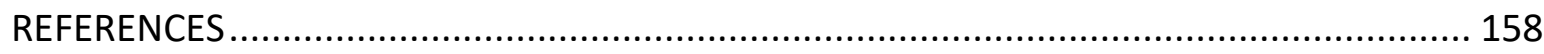




\section{MATERIALS AND METHODS}

Unless otherwise noted, all reactions were carried out under argon atmosphere in flame-dried glassware using standard syringe, cannula, and septa apparatus. Toluene, tetrahydrofuran, 1,4dioxane, acetonitrile, and N,N-dimethylformamide were dried with a dry solvent system before use. Unless otherwise stated, reagents were purchased from commercial sources and used without purification. Reactions were magnetically stirred and monitored by thin layer chromatography carried out on $0.25 \mathrm{~mm}$ silica gel plate (60f-254) using UV light as a visualizing agent, an ethanolic solution of $\mathrm{p}$-anisaldehyde and an aqueous solution of ceric ammonium molybdate, and heat as developing agents. Column chromatography was performed using Kieselgel 60 (70-230 mesh), Kieselgel 60 (230-400 mesh) or, for distannylarenes, Sorbtech 'Premium Rf' (200-400 mesh). Preparative thin-layer chromatography (PTLC) separations were carried out on 0.25 or $1.0 \mathrm{~mm}$ silica gel plates (60F-254). 1,4-dibromonaphthalene and 9,10-dibromoanthracene were purchased from commercial sources and used without further purification. 1,4-dibromoanthracene and 1,5dibromoanthracene were prepared following literature procedure. ${ }^{1}$

- AIBN: $5 \mathrm{~g}$ of commercial AIBN (Aldrich) were mixed with $200 \mathrm{~mL}$ of regular methanol, the mixture was stirred for $1 \mathrm{~h}$, filtered and cooled to $0^{\circ} \mathrm{C}$ for $12 \mathrm{~h}$. White crystals were collected, dried under vacuum and stored under argon at $0^{\circ} \mathrm{C}$. Purity of the AIBN was confirmed by ${ }^{1} \mathrm{H}$ $\mathrm{NMR}$ in $\mathrm{CDCl}_{3}$.

- ACHN: $5 \mathrm{~g}$ of commercial ACHN (Aldrich) were mixed with $200 \mathrm{~mL}$ of regular methanol, the mixture was stirred for $1 \mathrm{~h}$, filtered and cooled to $0^{\circ} \mathrm{C}$ for $12 \mathrm{~h}$. White crystals were collected, dried under vacuum and stored under argon at $0^{\circ} \mathrm{C}$. Purity of the $\mathrm{ACHN}$ was confirmed by ${ }^{1} \mathrm{H} \mathrm{NMR}$ in $\mathrm{CDCl}_{3}$.

- $\mathrm{Bu}_{3} \mathrm{SnH}$ : commercial (EMD Millipore) $\mathrm{Bu}_{3} \mathrm{SnH}$ was distilled under vacuum (b.p. $=61^{\circ} \mathrm{C}$ at 0.35 Torr) and stored under argon at $0^{\circ} \mathrm{C}$. Purity of the $\mathrm{Bu}_{3} \mathrm{SnH}$ was confirmed by ${ }^{1} \mathrm{H}$ NMR both in $\mathrm{CDCl}_{3}$ and $\mathrm{C}_{6} \mathrm{D}_{6}$.

\section{Spectroscopic data:}

NMR spectra were recorded on Bruker $600 \mathrm{MHz}$ and Varian $400 \mathrm{MHz}$ instruments and calibrated using residual undeuterated solvents as an internal reference. ${ }^{13} \mathrm{C}$ NMR spectra were run at 150/100 $\mathrm{MHz}$. chemical shift $(\delta)$ is reported in ppm, $\mathrm{J}$ is the coupling constant and is expressed in $\mathrm{Hz}$. The following abbreviations were used to explain multiplicities: $s=$ singlet, $d=$ doublet, $t=$ triplet, $q=$ quartet, $\mathrm{dd}=$ doublet of doublets, $\mathrm{m}=$ multiplet, quin = quintet, sext = sextet, sep = septet, $\mathrm{br}=$ broad. High-resolution mass spectra (HRMS) were determined on an Agilent 6230 time-of-flight LC/MS (LC/TOF) system or an lonsense Direct Analysis in Real Time (DART MS). MALDI mass spectrometry was performed on a Bruker Autoflex III Smartbeam Mass Spectrometer (MALDI-TOF MS) in positive mode using a matrix of dithranol. 


\section{EXPERIMENTAL DATA}

Synthesis of dicarbaldehyde starting materials for the preparation of radical precursors 1a-j.

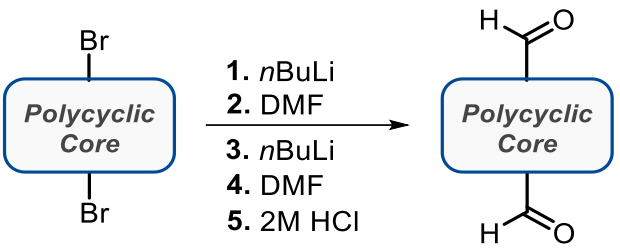

Naphthalene-1,4-dicarbaldehyde ( $\left.S_{i}\right)$ : A solution of 1,4-dibromonaphthalene $(5.0 \mathrm{~g}, 17.48 \mathrm{mmol})$ in tetrahydrofuran $(70 \mathrm{~mL})$ was cooled down to $-78^{\circ} \mathrm{C}$ in a dry ice/acetone bath. $8.0 \mathrm{~mL}$
of $n$-butyllithium $(19.23 \mathrm{mmol}, 1.1$ equiv. $)$ were added dropwise to the solution, and
the mixture was stirred at $-78^{\circ} \mathrm{C}$ for 1 hour. Next, $1.42 \mathrm{~mL}$ of $\mathrm{N}, \mathrm{N}$-dimethylformamide
$(18.36 \mathrm{mmol}, 1.05$ equiv.) was added to the reaction mixture dropwise, and the
mixture was brought to room temperature to stir for 30 minutes. After this time, the mixture was cooled down to $-78^{\circ} \mathrm{C}$ again and $18.2 \mathrm{~mL}$ of $n$-butyllithium ( $43.7 \mathrm{mmol}, 2.5$ equiv.) were added dropwise and left to stir for 2 hours at $-78^{\circ} \mathrm{C}$. Then, $4.1 \mathrm{~mL}$ of $\mathrm{N}, \mathrm{N}$-dimethylformamide $(52.45$ mmol, 3.0 equiv.) was added dropwise and the mixture was brought to room temperature to stir for 1 hour. Finally, the reaction was quenched by adding an excess of $2 \mathrm{M} \mathrm{HCl}$ and letting stir at room temperature for 30 minutes. The organic phase was washed with saturated ammonium chloride $(x 3)$ and brine (x3). The combined aqueous layer was extracted with $30 \mathrm{~mL}$ methylene chloride (x3), dried over $\mathrm{MgSO}_{4}$ and concentrated under vacuum. The spectroscopic data matched the reported data for this compound. The title compound was isolated by recrystallization from EtOAc/hexanes to yield $2.24 \mathrm{~g}$ of pale yellow solid (70\%). The compound can be stored for months in a $20 \mathrm{~mL}$ vial at $-20^{\circ} \mathrm{C}$ without any detectable loss in quality according to ${ }^{1} \mathrm{H}$ NMR spectroscopy.

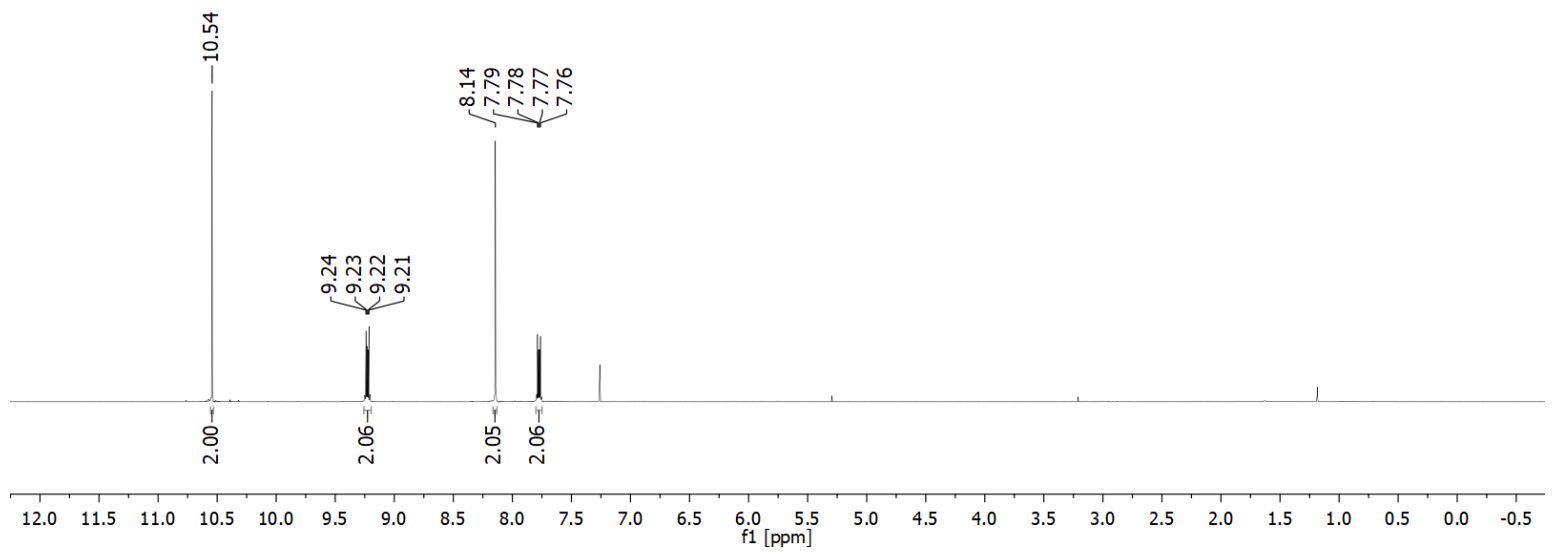

Figure S1. ${ }^{1} \mathrm{H} \mathrm{NMR}\left(400 \mathrm{MHz}, \mathrm{CDCl}_{3}\right)$ of compound $\mathbf{S}_{i}$. 
Anthracene-9,10-dicarbaldehyde ( $\left.S_{i i}\right)$ : A solution of 9,10-dibromoanthracene $(1.0 \mathrm{~g}, 2.98 \mathrm{mmol})$ in<smiles>O=Cc1c2ccccc2c(C=O)c2ccccc12</smiles>
tetrahydrofuran $(25 \mathrm{~mL})$ was cooled down to $-78^{\circ} \mathrm{C}$ in a dry ice/acetone bath. 1.36 $\mathrm{mL}$ of $n$-butyllithium ( $3.27 \mathrm{mmol}, 1.1$ equiv.) were added dropwise to the solution and the mixture was stirred at $-78^{\circ} \mathrm{C}$ for 1 hour. Next, $0.25 \mathrm{~mL}$ of $\mathrm{N}, \mathrm{N}-$ dimethylformamide ( $3.27 \mathrm{mmol}, 1.1$ equiv.) was added to the reaction mixture dropwise, and the mixture was brought to room temperature to stir for 30 minutes. After this time, the mixture was cooled to $-78^{\circ} \mathrm{C}$ again and $3.40 \mathrm{~mL}$ of $n$ butyllithium ( $8.48 \mathrm{mmol}, 2.85$ equiv.) were added dropwise and left to stir at $-78^{\circ} \mathrm{C}$ for 2 hours. Then, $1.15 \mathrm{~mL}$ of $\mathrm{N}, \mathrm{N}$-dimethylformamide ( $14.85 \mathrm{mmol}, 5.0$ equiv.) was added dropwise and the mixture was brought to room temperature to stir for 1 hour. Finally, the reaction mixture was poured into a 0.1 water. The precipitate was filtered and dried to afford $0.275 \mathrm{~g}$ of the title compound as a yellow solid (39\%). The spectroscopic data matched the reported data for this compound.

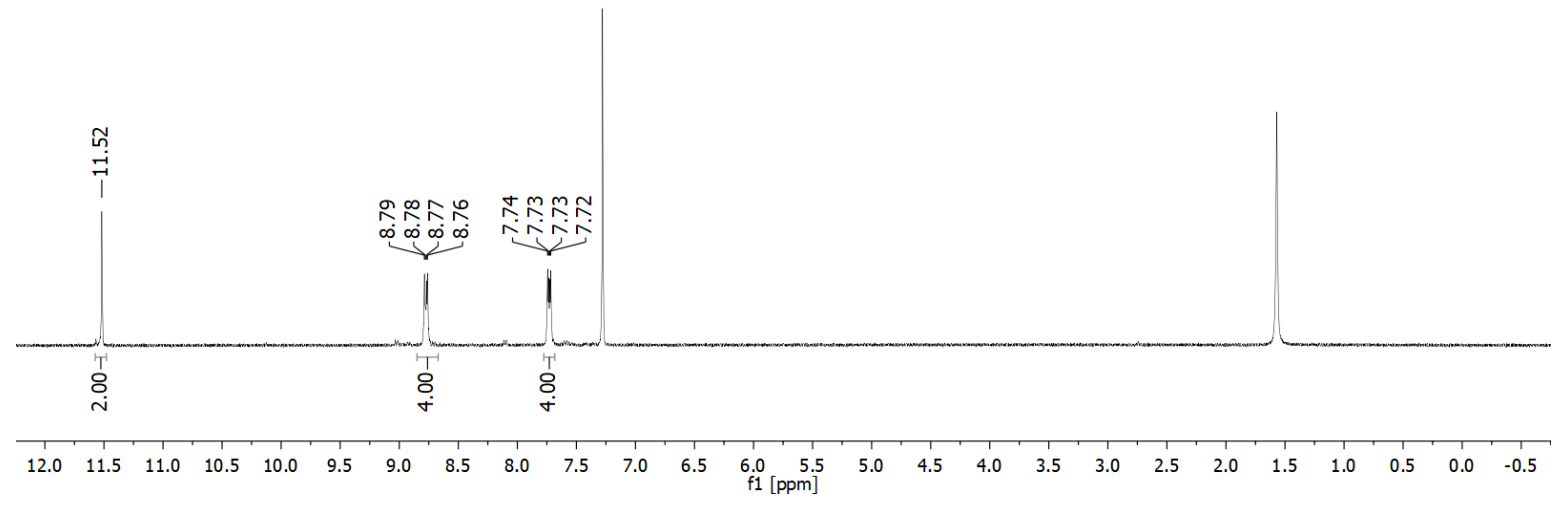

Figure S2. ${ }^{1} \mathrm{H} \mathrm{NMR}\left(400 \mathrm{MHz}, \mathrm{CDCl}_{3}\right)$ of compound $\boldsymbol{S}_{\text {ii. }}$.

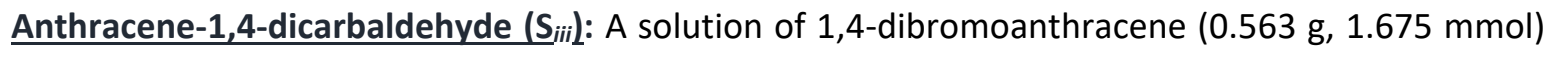<smiles>O=Cc1ccc(C=O)c2cc3ccccc3cc12</smiles>
in tetrahydrofuran $(8.4 \mathrm{~mL})$ was cooled down to $-78^{\circ} \mathrm{C}$ in a dry ice/acetone bath. $0.68 \mathrm{~mL}$ of $n$-butyllithium ( $1.675 \mathrm{mmol}, 1$ equiv.) were added dropwise to the solution, and the mixture was stirred at $-78^{\circ} \mathrm{C}$ for 1 hour. Next, $0.16 \mathrm{~mL}$ of $\mathrm{N}, \mathrm{N}$ dimethylformamide ( $2.09 \mathrm{mmol}, 1.25$ equiv.) was added to the reaction mixture dropwise and the mixture was brought to room temperature to stir for 30 minutes. After this time, the mixture was cooled to $-78^{\circ} \mathrm{C}$ again and $1.7 \mathrm{~mL}$ of $n$ butyllithium ( $4.185 \mathrm{mmol}, 2.5$ equiv.) were added dropwise and left to stir at $-78^{\circ} \mathrm{C}$ for 2 hours. Then, $0.46 \mathrm{~mL}$ of $\mathrm{N}, \mathrm{N}$-dimethylformamide $(5.99 \mathrm{mmol}, 3.6$ equiv.) was added dropwise and the mixture was brought to room temperature to stir for 1 hour. Finally, the reaction was quenched by adding an excess of $2 \mathrm{M} \mathrm{HCl}$ and letting stir at room temperature for 30 minutes. The organic phase was washed with saturated ammonium chloride $(x 3)$ and then brine $(x 3)$. The combined aqueous layer was then extracted with $30 \mathrm{~mL}$ methylene chloride (x3). The combined organic layer was dried over $\mathrm{MgSO}_{4}$ and concentrated under vacuum to afford $0.366 \mathrm{~g}$ of the title compound as a red-orange solid (93\%). The spectroscopic data matched the reported data for this compound. In some attempts, the product was observed to form a hydrate presented as an orange oil; this was converted back to the dialdehyde by concentrating the mixture from dry toluene $(x 4)$. 


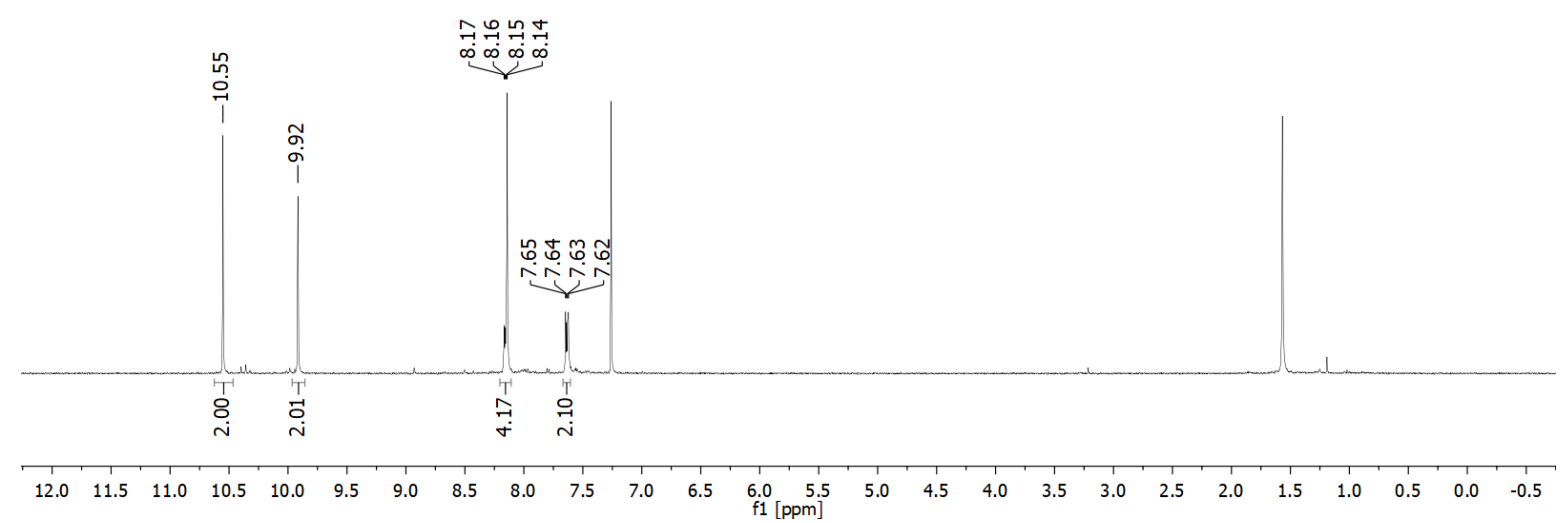

Figure S3. ${ }^{1} \mathrm{H}$ NMR $\left(400 \mathrm{MHz}, \mathrm{CDCl}_{3}\right)$ of compound $\boldsymbol{S}_{\text {iii. }}$.

Anthracene-1,5-dicarbaldehyde ( $\left.S_{i v}\right):$ Prepared by modifying a literature procedure. ${ }^{2} n$-butyl lithium<smiles>O=Cc1cccc2cc3ccccc3cc12</smiles>
(12.86 mL; $6.5 \mathrm{mmol}$ ) was added to a solution of 1,5-dibromoanthracene (2 g; $5.95 \mathrm{mmol})$ in dry ether $(80 \mathrm{~mL})$ after cooled to $-10^{\circ} \mathrm{C}$. The reaction mixture was stirred at $0^{\circ} \mathrm{C}$ for $30 \mathrm{~min}$ before it was warmed to ambient temperature. After $1 \mathrm{~h}$, the solution was cooled to $-78^{\circ} \mathrm{C}$ and $\mathrm{N}, \mathrm{N}$-dimethylformamide $(1.85 \mathrm{~mL}$; mmol) was added dropwise. The reaction was stirred for $1.5 \mathrm{~h}$, warmed to ambient temperature and diluted with water $(20 \mathrm{~mL})$. The resultant precipitate was filtered and washed with water. After washing, the residue was dissolved in methylene chloride and filtered leaving an impurity retained on the filter. Finally, the title compound was isolated by passing through a plug of silica with methylene chloride as eluent to give the title compound as a yellow crystalline solid ( $0.85 \mathrm{~g}, 61 \%$ yield).

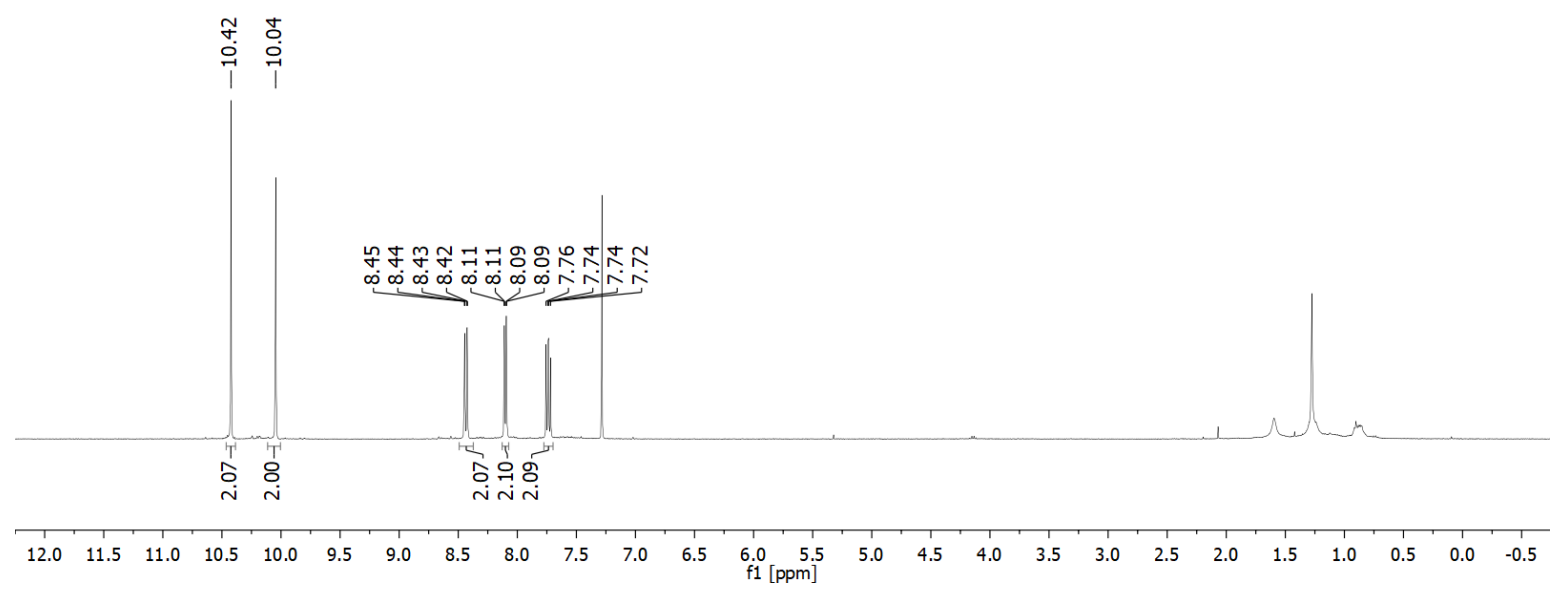

Figure S4. ${ }^{1} \mathrm{H}$ NMR (400 MHz, $\left.\mathrm{CDCl}_{3}\right)$ of compound $\boldsymbol{S}_{i v}$. 
4-bromo-1-naphthaldehyde ( $\mathrm{S}_{\mathrm{v}}$ ): A solution of $0.85 \mathrm{~g}$ of 1,4-dibromonaphthalene $(2.883 \mathrm{mmol})$ in the resulting bright yellow solution was left to stir at $-78 \mathrm{C}$ for $1.5 \mathrm{~h}$. After this time,
solution of $n$-butyllithium (2.883 mmol, 1.0 equiv.) in hexanes was added dropwise.
$0.267 \mathrm{~mL}(3.46 \mathrm{mmol} ; 1.2$ equiv.) of $\mathrm{N}, \mathrm{N}$-dimethylformamide were added slowly and
following addition the reaction was allowed to reach room temperature. The reaction was stirred at room temperature for an extra 45 minutes and at this point the reaction mixture was hydrolyzed with a solution of $2 \mathrm{M} \mathrm{HCl}$. After stirring for 30 minutes, the mixture was extracted with $30 \mathrm{~mL}$ diethyl ether $(x 3)$ and the combined organic layer was washed with brine. The solvent was removed under reduced pressure and purification by column chromatography using $2 \%$ ethyl acetate in hexanes as an eluent performed to isolate $0.583 \mathrm{~g}$ ( $86 \%$ yield) of title compound as a white solid.

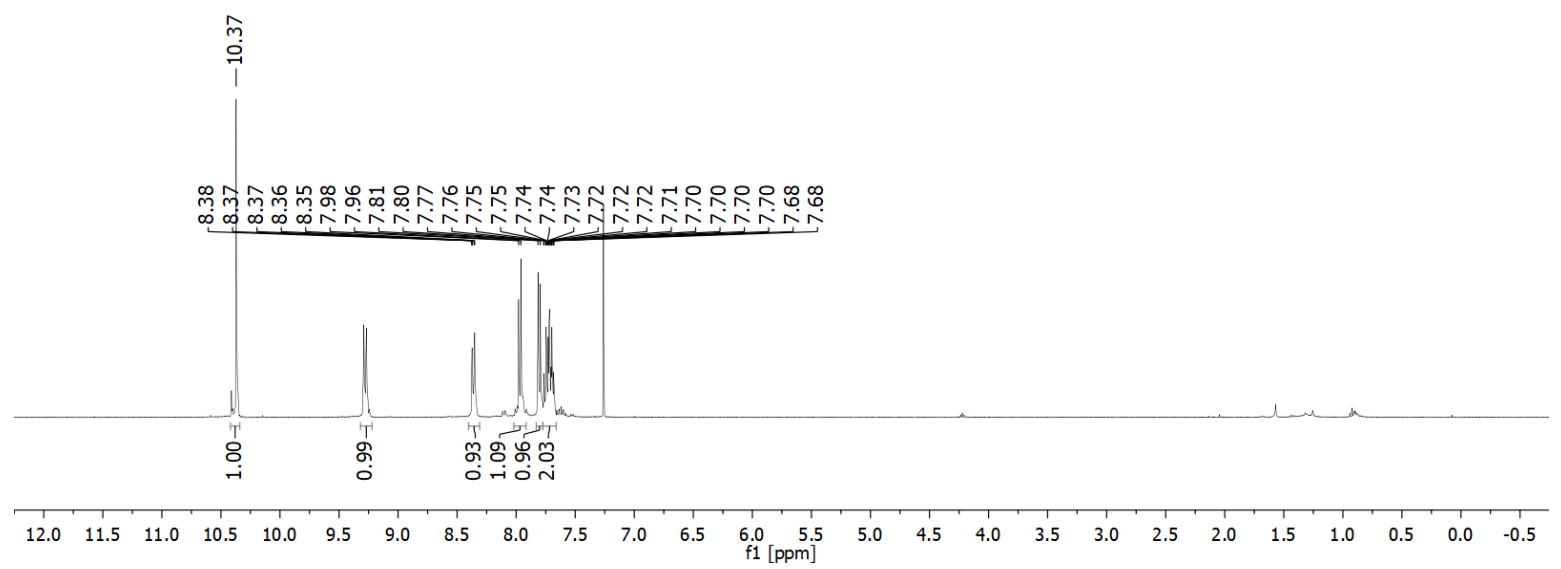

Figure S5. ${ }^{1} \mathrm{H}$ NMR $\left(400 \mathrm{MHz}, \mathrm{CDCl}_{3}\right)$ of compound $\mathbf{S}_{v}$.

4,4'-binaphthyl-1,1'-dicarbaldehyde (S Svil : $0.5 \mathrm{~g}$ of 4-bromo-1-naphthaldehyde (2.13 mmol), $0.26 \mathrm{~g}$<smiles>O=Cc1ccc(-c2ccc(C=O)c3ccccc23)c2ccccc12</smiles>
$B_{2} \operatorname{pin}_{2}$ (1.02 mmol; 0.48 equiv.), $0.078 \mathrm{~g} \mathrm{Pd}(\mathrm{dpppf}) \mathrm{Cl}_{2}$ ( $0.106 \mathrm{mmol} ; 0.05$ equiv.) and 0.8 g KOAc ( $8 \mathrm{mmol} ; 4$ equiv.) were added to a $50 \mathrm{~mL}$ round bottom flask equipped with a magnetic stir bar and a Vigreux condenser. The mixture of solids was dissolved in 11 $\mathrm{mL}$ of 1,4-dioxane and heated up to $90 \mathrm{C}$ for 20 hours. The reaction mixture was then filtered through a celite pad and the solvent removed under reduced pressure. The reaction crude was then dissolved in methylene chloride and washed with a saturated solution of $\mathrm{NH}_{4} \mathrm{Cl}(\mathrm{x} 3)$. The combined organic layer was collected, dried over $\mathrm{MgSO}_{4}$, and solvent removed. Given that the desired product is sparingly soluble in common organic solvents, and that its calculated purity in the crude was $\sim 93 \%$ (unreacted starting material and Bpin-AcOEt as impurities), this mixture was used in the next step without further purification. 


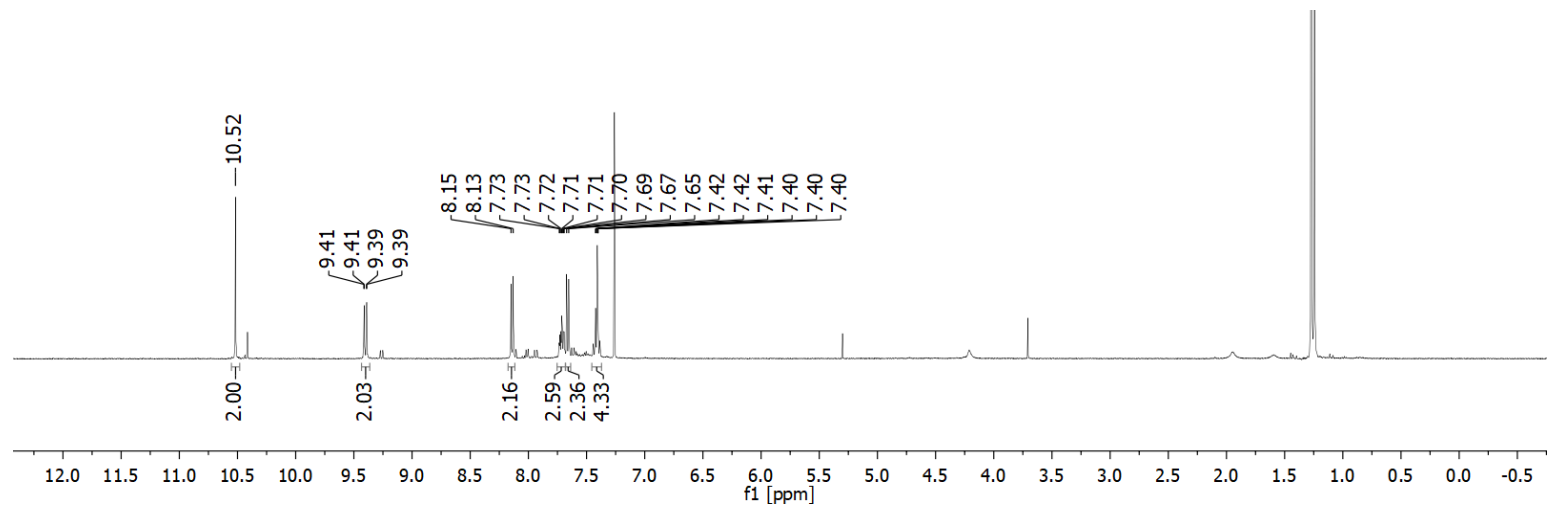

Figure S6. ${ }^{1} \mathrm{H}$ NMR $\left(400 \mathrm{MHz}, \mathrm{CDCl}_{3}\right)$ of crude compound $\boldsymbol{S}_{\mathrm{vi}}$. 
Synthesis of radical cyclization precursors (compounds $1 \mathrm{a}-\mathrm{j}$ )

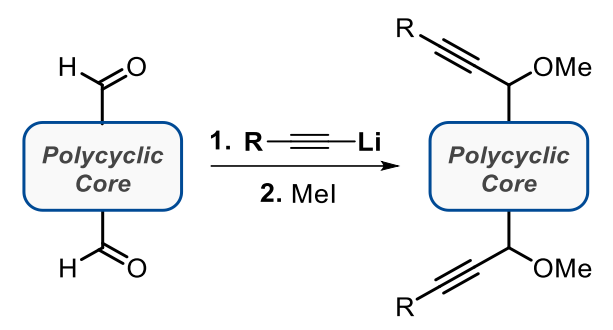

General procedure A:

A $0.43 \mathrm{M}$ solution of acetylene ( 2.2 equiv.) in THF was cooled to $-78^{\circ} \mathrm{C}$ in a dry ice/acetone bath. A volume corresponding to 2.2 equivalents of $n$-butyllithium solution in hexanes were added dropwise. The reaction mixture was kept in the bath for 1 hour. Next, a solution of the corresponding aryldicarbaldehyde starting material (1.0 equiv.) in THF was added dropwise to the freshly formed lithium acetylide mixture. When addition was finalized, the reaction mixture was brought to room temperature and stirred for $1.5 \mathrm{~h}$. After this time, methyl iodide (6.0 equiv.) was added slowly and the mixture was stirred overnight. The solvent was removed under reduced pressure and the crude reaction mixture redissolved in methylene chloride. The solution was washed with brine and the aqueous phase was extracted with methylene chloride. The combined organic phase was dried over $\mathrm{Na}_{2} \mathrm{SO}_{4}$ and concentrated under vacuum. Purification was performed by column chromatography in all cases using a mixture of EtOAc-Hexanes or $\mathrm{Et}_{2} \mathrm{O}$-Hexanes as the mobile phase. All the products are present as a diastereomeric mixtures causing, in some instances, widened or overcomplicated spectral features. For the same reason, some samples may show "doubled" ${ }^{13} \mathrm{C}$ NMR signals.

1,4-bis(1-methoxyhept-2-yn-1-yl)naphthalene (compound 1a). Following general procedure A, the

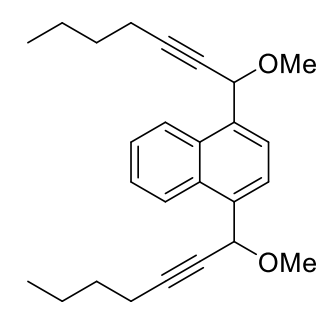
title compound was synthesized from $0.4 \mathrm{~g}(2.17 \mathrm{mmol})$ of naphthalene-1,4dicarboxaldehyde (compound $\boldsymbol{S}_{i}$; see previous section) and 1-hexyne. The product was isolated by column chromatography using $5 \% \mathrm{Et}_{2} \mathrm{O}$ in hexanes as eluent to give $0.325 \mathrm{~g}$ of desired compound as a light-yellow oil (88\%).

${ }^{1} \mathrm{H}$ NMR $\left(400 \mathrm{MHz}, \mathrm{CDCl}_{3}, \delta\right): 8.38-8.35(\mathrm{~m}, 2 \mathrm{H}), 7.82(\mathrm{~s}, 2 \mathrm{H}), 7.60-7.56(\mathrm{~m}$, $2 \mathrm{H}), 5.74(\mathrm{~s}, 2 \mathrm{H}), 3.49(\mathrm{~s}, 6 \mathrm{H}), 2.33(\mathrm{t}, J=7.0 \mathrm{~Hz}, 4 \mathrm{H}), 1.59-1.44(\mathrm{~m}, 8 \mathrm{H}), 0.95$

(t, $J=7.3 \mathrm{~Hz}, 6 \mathrm{H}$ ).

${ }^{13} \mathrm{C} \mathrm{NMR}\left(150 \mathrm{MHz}, \mathrm{CDCl}_{3}, 8\right): 135.2,131.5,125.9,125.1,125.0,124.89,89.3,71.7,55.6,30.7,22.0$, 18.6, 13.6.

HRMS (DART, positive, $\mathrm{m} / \mathrm{z}$ ): calcd. for $\mathrm{C}_{26} \mathrm{H}_{31} \mathrm{O}_{2}{ }^{+}[\mathrm{M}-\mathrm{H}]^{+}: 375.2324$; found: 375.2345 . 
1,4-bis(1-methoxy-3-phenylprop-2-yn-1-yl)naphthalene (compound $\mathbf{1 b}$ ). Following general

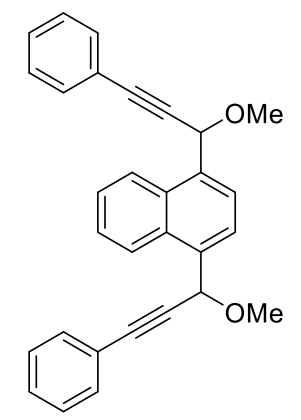
procedure $A$, the title compound was synthesized from $0.8 \mathrm{~g}(4.34 \mathrm{mmol})$ of naphthalene-1,4-dicarboxaldehyde (compound $\mathbf{S}_{i}$; see previous section) and phenylacetylene. The product was isolated by column chromatography using $8 \% \mathrm{Et}_{2} \mathrm{O}$ in hexanes as eluent to give $1.41 \mathrm{~g}$ of desired compound as a lightyellow oil (78\%).

${ }^{1} \mathrm{H}$ NMR $\left(400 \mathrm{MHz}, \mathrm{CDCl}_{3}, 8\right): 8.42-8.39(\mathrm{~m}, 2 \mathrm{H}), 7.88(\mathrm{~s}, 2 \mathrm{H}), 7.63-7.60(\mathrm{~m}, 2 \mathrm{H})$, 7.50-7.48 (m, 4H), 7.34-7.30 (m, 6H), $5.96(\mathrm{~s}, 2 \mathrm{H}), 3.56(\mathrm{~s}, 6 \mathrm{H})$.

${ }^{13} \mathrm{C} \mathrm{NMR}\left(150 \mathrm{MHz}, \mathrm{CDCl}_{3}, \delta\right): 135.2,132.1,131.8,128.9,128.6,126.6,125.6$, $125.1,122.9,88.8,86.8,72.4,72.3,56.3$.

HRMS (ESI, positive, $\mathrm{m} / \mathrm{z}$ ): calcd. for $\mathrm{C}_{30} \mathrm{H}_{24} \mathrm{O}_{2} \mathrm{Na}^{+}[\mathrm{M}+\mathrm{Na}]^{+}:$439.1674; found: 439.1681 .

1,4-bis(1-methoxy-3-(naphthalen-1-yl)prop-2-yn-1-yl)naphthalene (compound 1c). Following<smiles>COC(C#Cc1cccc2ccccc12)c1ccc(C(C#Cc2cccc3ccccc23)OC)c2ccccc12</smiles>
general procedure $A$, the title compound was synthesized from $0.2 \mathrm{~g}(1.085$ $\mathrm{mmol}$ ) of naphthalene-1,4-dicarboxaldehyde (compound $\boldsymbol{S}_{i}$; see previous section) and 1-ethynylnaphthalene. The product was isolated by column chromatography using $10 \% \mathrm{Et}_{2} \mathrm{O}$ in hexanes as eluent to give $0.431 \mathrm{~g}$ of desired compound as a white resin (77\%).

${ }^{1} \mathrm{H}$ NMR $\left(400 \mathrm{MHz} \mathrm{CDCl}_{3}, \delta\right): 8.58-8.54(\mathrm{~m}, 2 \mathrm{H}), 8.34-8.32(\mathrm{~m}, 2 \mathrm{H}), 8.02-8.00$ $(\mathrm{m}, 2 \mathrm{H}), 7.87-7.83(\mathrm{~m}, 4 \mathrm{H}), 7.76-7.73(\mathrm{~m}, 2 \mathrm{H}), 7.71-7.67(\mathrm{~m}, 2 \mathrm{H}), 7.56-7.49$ $(\mathrm{m}, 4 \mathrm{H}), 7.46-7.41(\mathrm{~m}, 2 \mathrm{H}), 6.14(\mathrm{~s}, 2 \mathrm{H}), 3.68(\mathrm{~s}, 6 \mathrm{H})$ (the presence of two atropisomers with overlapping signals complicates the spectral features).

$\left.{ }^{13} \mathrm{C} \mathrm{NMR} \mathrm{(150} \mathrm{MHz,} \mathrm{CDCl} 3,8\right): 135.0,133.4,133.1,131.6,130.8,129.0,128.3,126.9,126.5,126.4$, $126.1,125.5,125.4,125.1,125.0,120.2,31.4,86.73,72.4,56.1$. (an "extra" signal is found in the aromatic region due to the presence of two atropisomers).

HRMS (ESI, positive, $\mathrm{m} / \mathrm{z}$ ): calcd. for $\mathrm{C}_{38} \mathrm{H}_{27} \mathrm{O}_{2}{ }^{+}[\mathrm{M}-\mathrm{H}]^{+}$: 515.2011; found: 515.2000 .

1,4-bis(1-methoxy-3-(4-methoxyphenyl)prop-2-yn-1-yl)naphthalene (compound 1d). Following

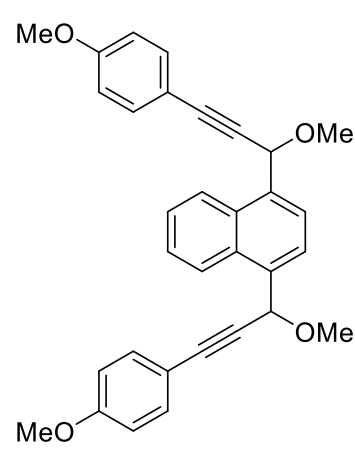
general procedure $A$, the title compound was synthesized from $0.4 \mathrm{~g}$ ( 2.17 $\mathrm{mmol}$ ) of naphthalene-1,4-dicarboxaldehyde (compound $\mathbf{S}_{i}$; see previous section) and 4-ethynylanisole. The product was isolated by column chromatography using 15\% EtOAc in hexanes as eluent to give $0.9 \mathrm{~g}$ of desired compound as a light-yellow oil (87\%).

${ }^{1} \mathrm{H}$ NMR (400 MHz, $\left.\mathrm{CDCl}_{3}, \delta\right):$ 8.42-8.39 (m, 2H), $7.87(\mathrm{~s}, 2 \mathrm{H}), 7.62-7.59$ $(\mathrm{m}, 2 \mathrm{H}), 7.43-7.41(\mathrm{~m}, 4 \mathrm{H}), 6.86-6.83(\mathrm{~m}, 4 \mathrm{H}), 5.93(\mathrm{~s}, 2 \mathrm{H}), 3.80(\mathrm{~s}, 6 \mathrm{H})$, $3.54(\mathrm{~s}, 6 \mathrm{H})$.

${ }^{13} \mathrm{C}$ NMR (150 MHz, $\left.\mathrm{CDCl}_{3}, \delta\right): 159.8,134.9,133.3,131.5,126.1,125.3$, 124.8, 114.6, 113.9, 88.4, 85.0, 72.0, 55.8, 55.3.

HRMS (DART, positive, m/z): calcd. for $\mathrm{C}_{32} \mathrm{H}_{27} \mathrm{O}_{4}{ }^{+}[\mathrm{M}-\mathrm{H}]^{+}: 475.1909$; found: 475.1917 . 
4,4'-(naphthalene-1,4-diylbis(3-methoxyprop-1-yne-3,1-diyl))dibenzonitrile (compound 1e).

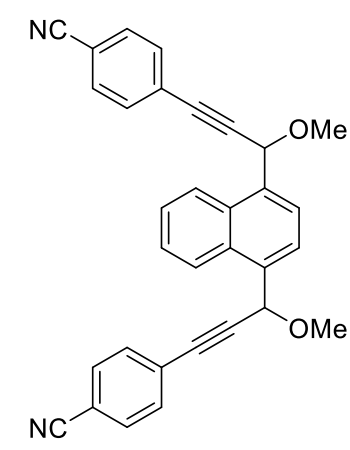

Following general procedure $A$, the title compound was synthesized from $0.4 \mathrm{~g}$ ( $2.17 \mathrm{mmol}$ ) of naphthalene-1,4-dicarboxaldehyde (compound $\mathbf{S}_{i}$; see previous section) and 4-ethynylbenzonitrile. The product was isolated by column chromatography using $20 \%$ EtOAc-Hexanes to give $0.815 \mathrm{~g}$ of desired compound as a light-yellow oil (80\%).

${ }^{1} \mathrm{H} \mathrm{NMR}\left(400 \mathrm{MHz}, \mathrm{CDCl}_{3}, \delta\right): 8.38-8.36(\mathrm{~m}, 2 \mathrm{H}), 7.81(\mathrm{~s}, 2 \mathrm{H}), 7.64-7.59(\mathrm{~m}$, $6 \mathrm{H}), 7.54-7.52(\mathrm{~m}, 4 \mathrm{H}), 5.92(\mathrm{~s}, 2 \mathrm{H}), 3.56(\mathrm{~s}, 6 \mathrm{H})$.

${ }^{13} \mathrm{C}$ NMR (150 MHz, $\left.\mathrm{CDCl}_{3}, \delta\right): 134.5,132.3,132.0,131.3,127.3,126.5$, 125.2, 124.7, 118.4, 112.0, 91.0, 86.6, 72.0, 56.3.

HRMS (DART, positive, $\mathrm{m} / \mathrm{z}$ ): calcd. for $\mathrm{C}_{32} \mathrm{H}_{26} \mathrm{~N}_{3} \mathrm{O}_{2}{ }^{+}\left[\mathrm{M}+\mathrm{NH}_{4}\right]^{+}$: 484.2025; found: 484.2039.

9,10-bis(1-methoxy-3-phenylprop-2-yn-1-yl)anthracene (compound 1f). Following general

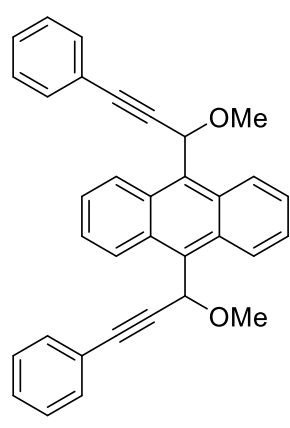
procedure $A$, the title compound was synthesized from $0.15 \mathrm{~g}(0.814 \mathrm{mmol})$ of anthracene-9,10-dicarboxaldehyde (compound $\boldsymbol{S}_{i i}$; see above) and phenylacetylene. The product was isolated by column chromatography using $8 \% \mathrm{Et}_{2} \mathrm{O}-$ Hexanes as eluent to give $0.236 \mathrm{~g}$ of desired compound as a lightyellow oil (79\%).

${ }^{1} \mathrm{H}$ NMR $\left(400 \mathrm{MHz}, \mathrm{CDCl}_{3}, 8\right):$ 8.82-8.79 (m, 4H), 7.59-7.56 (m, $\left.4 \mathrm{H}\right), 7.38-7.36$ $(\mathrm{m}, 4 \mathrm{H}), 7.26(\mathrm{~m}, 6 \mathrm{H}), 6.73(\mathrm{~m}, 2 \mathrm{H}), 3.58-3.56(\mathrm{~m}, 6 \mathrm{H})$.

${ }^{13} \mathrm{C}$ NMR (150 MHz, $\left.\mathrm{CDCl}_{3}, \delta\right): 131.7,131.3,131.3,129.6,128.4,128.1,125.5$, $125.4,125.4,122.6,122.6,88.2,88.2,87.8,87.8,68.5,68.5,57.0,57.0$.

MS (MALDI-TOF, positive, $\mathrm{m} / \mathrm{z}$ ): calcd. for $\mathrm{C}_{41} \mathrm{H}_{39} \mathrm{O}^{+}[\mathrm{M}-\mathrm{MeOH}]^{+}:$547.2995; found: 547.011.

1,4-bis(3-(4-(tert-butyl)phenyl)-1-methoxyprop-2-yn-1-yl)anthracene (compound 1g). Following

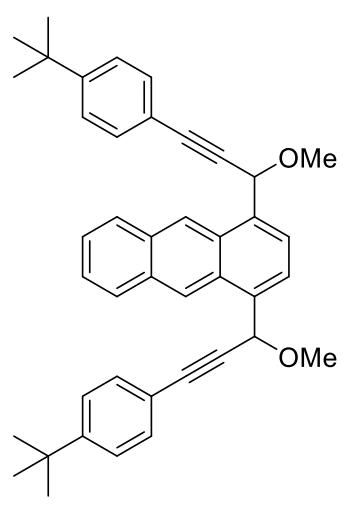

general procedure $A$, the title compound was synthesized from $0.45 \mathrm{~g}$ (1.92 $\mathrm{mmol}$ ) of anthracene-1,4-dicarboxaldehyde (compound $\boldsymbol{S}_{\text {iiii; }}$ see previous section) and 4-tert-butylphenylacetylene. The product was isolated by column chromatography using $5 \%$ EtOAc-Hexanes to give $0.68 \mathrm{~g}$ of desired compound as a light-yellow oil (61\%).

${ }^{1} \mathrm{H}$ NMR (400 MHz, $\left.\mathrm{CDCl}_{3}, 8\right)$ : 8.99-8.98 (br, 2H), 8.09-8.07 (m, 2H), 7.85 (s, $2 \mathrm{H}), 7.43(\mathrm{~d}, J=8.5 \mathrm{~Hz}, 4 \mathrm{H}), 7.33(\mathrm{~d}, J=8.5 \mathrm{~Hz}, 4 \mathrm{H}), 6.09(\mathrm{~s}, 2 \mathrm{H}), 3.57$ (s, $6 \mathrm{H}), 1.30(\mathrm{~s}, 18 \mathrm{H})$.

${ }^{13} \mathrm{C}$ NMR (150 MHz, $\left.\mathrm{CDCl}_{3}, \delta\right): 151.8,134.8,131.5$ 131.4, 129.5, 128.5, $125.8,125.3,124.9,123.9,119.5,88.6,85.8,72.3,72.3,55.6,34.7,31.1$.

MS (MALDI-TOF, positive, m/z): calcd. for $\mathrm{C}_{41} \mathrm{H}_{39} \mathrm{O}^{+}[\mathrm{M}-\mathrm{MeOH}]^{+}$: 547.2995; found: 547.031. 
1,5-bis(3-(4-(tert-butyl)phenyl)-1-methoxyprop-2-yn-1-yl)anthracene (compound $\mathbf{1 h}$ ). Following

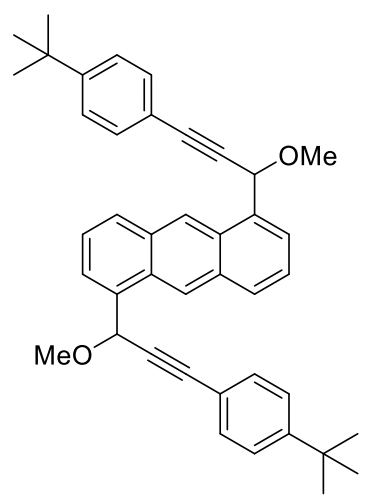
general procedure $A$, the title compound was synthesized from $0.27 \mathrm{~g}$ $(1.15 \mathrm{mmol})$ anthracene-1,5-dicarboxaldehyde $\left(\boldsymbol{S}_{i v}\right)$ and 4-tertbutylphenylacetylene. The product was isolated by column chromatography using 7\% EtOAc-Hexanes to give $0.3 \mathrm{~g}$ of desired compound as a light-yellow oil (45\%).

${ }^{1} \mathrm{H}$ NMR (400 MHz, $\left.\mathrm{CDCl}_{3}, \delta\right): 9.00(\mathrm{~s}, 2 \mathrm{H}), 8.12(\mathrm{~d}, J=8.0 \mathrm{~Hz}, 2 \mathrm{H}), 7.89$ (d, $J=8.0 \mathrm{~Hz}, 2 \mathrm{H}), 7.50(\mathrm{t}, J=8.0 \mathrm{~Hz}, 2 \mathrm{H}), 7.46(\mathrm{~d}, J=7.4 \mathrm{~Hz}, 4 \mathrm{H}), 7.35(\mathrm{~d}, J$ $=7.4 \mathrm{~Hz}, 4 \mathrm{H}), 6.11(\mathrm{~s}, 2 \mathrm{H}), 3.59(\mathrm{~s}, 6 \mathrm{H}), 1.32(\mathrm{~s}, 18 \mathrm{H})$.

${ }^{13} \mathrm{C}$ NMR (150 MHz, $\left.\mathrm{CDCl}_{3}, \delta\right): 151.8,133.4,132.4,131.5,130.5,128.9$, 126.0, 125.3, 124.6, 124.1, 119.6, 88.6, 85.9, 72.3, 55.6, 34.8, 31.1.

MS (MALDI-TOF, positive, m/z): calcd. for $\mathrm{C}_{41} \mathrm{H}_{39} \mathrm{O}^{+}[\mathrm{M}-\mathrm{MeOH}]^{+}$: 547.2995; found: 546.958.

4,4'-bis(1-methoxy-3-phenylprop-2-yn-1-yl)-1,1'-binaphthalene (compound 1i). Following general<smiles>COC(C#Cc1ccccc1)c1ccc(-c2ccc(C(C#Cc3ccccc3)OC)c3ccccc23)c2ccccc12</smiles>
procedure $A$, the title compound was synthesized from phenylacetylene and a crude sample of $4,4^{\prime}$-binaphthyl-1,1'-dicarbaldehyde (compound $\mathbf{S}_{v i} ; 93 \%$ purity calculated by NMR) prepared from $0.5 \mathrm{~g}$ of 4-bromo-1-naphthaldehyde (compound $\mathbf{S}_{v}, 2.13 \mathrm{mmol}$ ). The product was isolated by column chromatography using $10 \%$ EtOAc-Hexanes to give $0.48 \mathrm{~g}$ of desired compound as a light-yellow oil (2 steps, 73\% from 4-bromo-1-naphthaldehyde).

${ }^{1} \mathrm{H}$ NMR (400 MHz, $\left.\mathrm{CDCl}_{3}, \delta\right):$ 8.49-8.44 (m, 2H), 8.03-8.00 (m, 2H), 7.61-7.47 $(\mathrm{m}, 10 \mathrm{H}), 7.36-7.31(\mathrm{~m}, 8 \mathrm{H}), 6.07(\mathrm{~s}, 2 \mathrm{H}), 3.70(\mathrm{~s}, 3 \mathrm{H}), 3.68(\mathrm{~s}, 3 \mathrm{H})$.

${ }^{13} \mathrm{C} \mathrm{NMR}\left(150 \mathrm{MHz}, \mathrm{CDCl}_{3}, \delta\right): 139.8,139.8,133.6,133.5,133.4,131.9,131.0$, $131.0,128.6,128.3,127.5,127.4,127.2,127.1,126.3,126.0,125.6,125.5$, $124.4,124.3,122.6,88.6,86.6,72.2,72.1,56.3,56.1$.

MS (MALDI-TOF, positive, m/z): calcd. for $\mathrm{C}_{40} \mathrm{H}_{31} \mathrm{O}_{2}{ }^{+}[\mathrm{M}+\mathrm{H}]^{+}:$543.2324; found: 546.97.

1,4-bis(3-(4-(tert-butyl)phenyl)-1-methoxyprop-2-yn-1-yl)naphthalene (compound 1j). Following

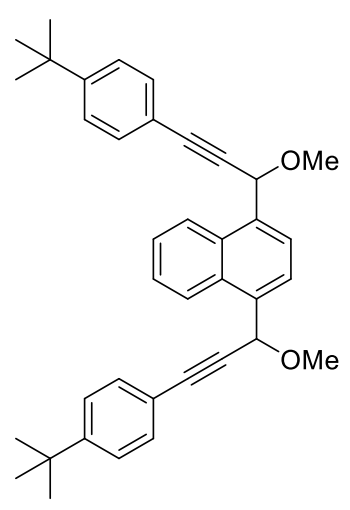
general procedure $A$, the title compound was synthesized from $0.7 \mathrm{~g}$ (3.80 mmol) of naphthalene-1,4-dicarboxaldehyde (compound $\boldsymbol{S}_{i}$; see above) and 4-tert-butylphenylacetylene. The product was isolated by column chromatography using $10 \% \mathrm{Et}_{2} \mathrm{O}-$ Hexanes to give $1.52 \mathrm{~g}$ of desired compound as a light-yellow oil (76\%).

${ }^{1} \mathrm{H}$ NMR (400 MHz, $\left.\mathrm{CDCl}_{3}, 8\right): 8.46-8.44(\mathrm{~m}, 2 \mathrm{H}), 7.93(\mathrm{~s}, 2 \mathrm{H}), 7.65-7.63(\mathrm{~m}$, $2 \mathrm{H}), 7.48(\mathrm{~d}, J=8.2 \mathrm{~Hz}, 4 \mathrm{H}), 7.38(\mathrm{~d}, J=8.2 \mathrm{~Hz}, 4 \mathrm{H}), 6.00(\mathrm{~s}, 2 \mathrm{H}), 3.59(\mathrm{~s}$, $6 \mathrm{H}), 1.35(\mathrm{~s}, 18 \mathrm{H})$.

${ }^{13} \mathrm{C}$ NMR (150 MHz, $\left.\mathrm{CDCl}_{3}, \delta\right): 151.9,135.0,131.6,126.3,125.4,125.4$, $124.9,124.9,119.6,88.7,85.9,72.1,55.9,34.9,31.2$.

HRMS (DART, positive, m/z): calcd. for $\mathrm{C}_{38} \mathrm{H}_{39} \mathrm{O}_{2}{ }^{+}[\mathrm{M}-\mathrm{H}]^{+}:$527.2950; found: 527.2961. 


\section{Fragmentation Mechanism for $[\mathrm{M}-\mathrm{H}]^{+}$}

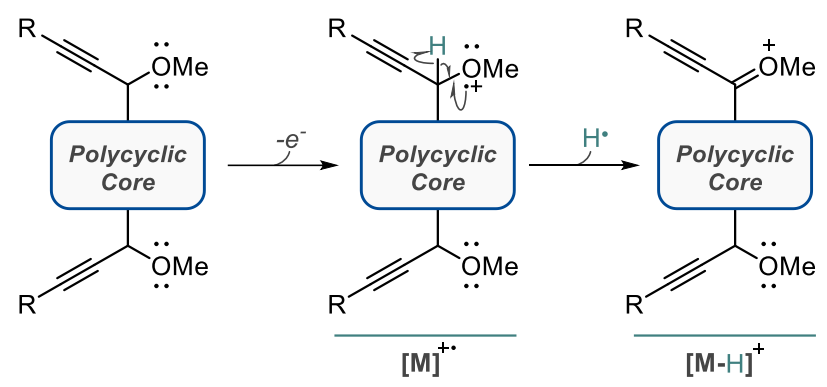

Figure S7. Proposed fragmentation mechanism of $[\mathrm{M}-\mathrm{H}]^{+}$observed for DART-MS of compounds 1a, 1c, $1 \mathbf{d}$ and $\mathbf{1 j}$.

Double alkyne radical peri-annulations (compounds 2a-j)

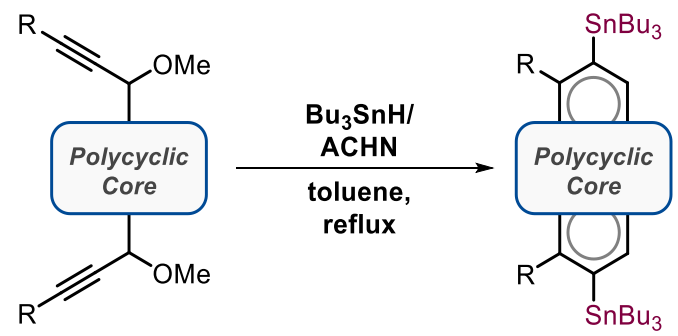

\section{General procedure B:}

A $0.31 \mathrm{M}$ solution of tributyltin hydride (2.5 equiv.) with 1.6 equivalents of $\mathrm{ACHN}$ was added via syringe pump over the course of 3 hours to a refluxing $0.02 \mathrm{M}$ solution of precursors $1 \mathrm{a}-\mathrm{j}$ in dry, outgassed toluene under argon atmosphere. The reaction mixture was then stirred for an additional 1.5 hours. The reaction was then allowed to cool to ambient temperature and toluene was removed under reduced pressure. A measured amount of dibromomethane was added as an internal standard, and a ${ }^{1} \mathrm{H}$ NMR was obtained to calculate yields. Purification was performed via column chromatography redissolving the crude reaction mixtures in hexanes and 'wet-loading' onto the stationary phase ('premium Rf' silica; pH 6.0 - 7.0; see materials and methods).

(1,8-dibutylpyrene-2,7-diyl)bis(tributylstannane) (compound 2a). Following general procedure B,<smiles>CCCCc1cc2ccc3cc(SCCC)c(CCCC)c4ccc(c1CCCC)c2c34</smiles>
the title compound was synthesized from $0.3 \mathrm{~g}(0.7967 \mathrm{mmol})$ of precursor 1a. The product was isolated by column chromatography using hexanes as eluent to give $0.3 \mathrm{~g}$ of a clear yellow oil (42\%).

${ }^{1} \mathrm{H}$ NMR (400 MHz, $\mathrm{CDCl}_{3}, \delta$ ): 8.32 (s accompanied with d peak with ${ }^{3} \mathrm{~J}_{\mathrm{H}-\mathrm{Sn}}=44.1$ $\mathrm{Hz}, 2 \mathrm{H}), 8.21(\mathrm{~s}, 2 \mathrm{H}), 7.98(\mathrm{~s}, 2 \mathrm{H}), 3.39(\mathrm{~b}, 4 \mathrm{H}), 1.67(\mathrm{~m}, 16 \mathrm{H}), 1.45$ (sext, $J=7.3$ $\mathrm{Hz}, 12 \mathrm{H}), 1.30(\mathrm{~m}, 12 \mathrm{H}), 1.13(\mathrm{t}, J=7.3 \mathrm{~Hz}, 6 \mathrm{H}), 0.97(\mathrm{t}, J=7.3 \mathrm{~Hz}, 18 \mathrm{H})$.

${ }^{13} \mathrm{C}$ NMR (150 MHz, $\left.\mathrm{CDCl}_{3}, \delta\right): 143.5,140.5,133.2,129.4,127.8,126.5,125.7$,

$123.7,37.6,35.3,29.3,27.5,23.7,14.2,13.7,10.8$. 
MS (MALDI-TOF, positive, m/z): calcd. for $\mathrm{C}_{48} \mathrm{H}_{78} \mathrm{Sn}_{2}{ }^{+}[\mathrm{M}]^{+}:$: 892.4142; found: 892.397.

(1,8-diphenylpyrene-2,7-diyl)bis(tributylstannane) (compound 2b). Following general procedure

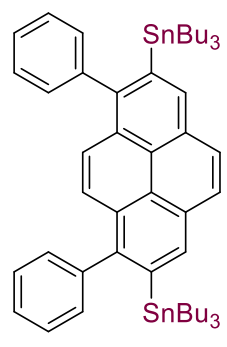
$\mathrm{B}$, the title compound was synthesized from $0.15 \mathrm{~g}(0.3601 \mathrm{mmol})$ of precursor $\mathbf{1 b}$. The product was isolated by column chromatography using hexanes as eluent to give $0.128 \mathrm{~g}$ of a clear oil (38\%).

${ }^{1} \mathrm{H}$ NMR (400 MHz, $\mathrm{CDCl}_{3}, \delta$ ): 8.32 (s accompanied with d peak with ${ }^{3} \mathrm{JH}_{\mathrm{H}-\mathrm{Sn}}=41.6$ $\mathrm{Hz}, 2 \mathrm{H}), 8.11(\mathrm{~s}, 2 \mathrm{H}), 7.50-7.39(\mathrm{~m}, 10 \mathrm{H}), 1.50-1.36(\mathrm{~m}, 12 \mathrm{H}), 1.28$ (sext, $J=7.3$ $\mathrm{Hz}, 12 \mathrm{H}), 0.88-0.75(\mathrm{~m}, 30 \mathrm{H})$.

${ }^{13} \mathrm{C}$ NMR (150 MHz, $\left.\mathrm{CDCl}_{3}, \delta\right): 145.0,143.5,141.0,132.7,130.7,129.9,128.4$, 128.0, 127.2, 127.1, 125.5, 124.6, 29.1, 27.3, 13.6, 13.7, 10.5.

MS (MALDI-TOF, positive, m/z): calcd. for $\mathrm{C}_{48} \mathrm{H}_{61} \mathrm{Sn}_{2}{ }^{+}\left[\mathrm{M}-\mathrm{C}_{4} \mathrm{H}_{9}\right]^{+}:$: 875.2811; found: 875.336 .

(1,8-di(naphthalen-1-yl)pyrene-2,7-diyl)bis(tributylstannane) (compound 2c). Following general

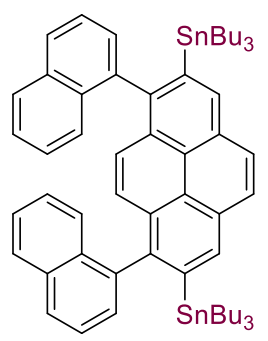
procedure $B$, the title compound was synthesized from $0.37 \mathrm{~g}(0.716 \mathrm{mmol})$ of precursor 1c. The product was isolated by column chromatography using hexanes as eluent to give $0.23 \mathrm{~g}$ of the title compound as a clear oil (31\%).

${ }^{1} \mathrm{H} \mathrm{NMR}\left(400 \mathrm{MHz}, \mathrm{CDCl}_{3}, \delta\right): 8.50$ (s accompanied with d peak with ${ }^{3} \mathrm{JH}_{\mathrm{H}-\mathrm{Sn}}=41.0$ $\mathrm{Hz}, 2 \mathrm{H}), 8.28(\mathrm{~s}, 2 \mathrm{H}), 7.97-7.92(\mathrm{~m}, 4 \mathrm{H}), 7.61(\mathrm{t}, J=7.4 \mathrm{~Hz}, 2 \mathrm{H}), 7.54-7.49(\mathrm{~m}$, $2 \mathrm{H}), 7.47-7.43(\mathrm{~m}, 2 \mathrm{H}), 7.30-7.21(\mathrm{~m}, 6 \mathrm{H}), 1.32-1.26(\mathrm{~m}, 12 \mathrm{H}), 1.23-1.14(\mathrm{~m}$, $12 \mathrm{H}), 0.84(\mathrm{t}, J=7.2 \mathrm{~Hz}, 18 \mathrm{H}) 0.71-0.56(\mathrm{~m}, 12 \mathrm{H})$.

${ }^{13} \mathrm{C}$ NMR (150 MHz, CDCl, 8): 200.2, 200.5, 200.07, 200.06, 199.7, 199.7, 197.8, 197.8, 197.8, 197.7, 197.7, 197.0, 197.0, 196.8, 196.8, 196.5, 196.5, 196.4, 196.4, 196.4, 196.3, 196.2, 196.2, 195.9, 195.9, 195.9, 195.9, 195. 9, 195.7, 195.6, 171.7, 171.5, 171.1, 167.7, 166.8, 166.8 ("extra" signals found caused by mixture of atropisomers).

MS (MALDI-TOF, positive, m/z): calcd. for $\mathrm{C}_{56} \mathrm{H}_{65} \mathrm{Sn}_{2}{ }^{+}\left[\mathrm{M}-\mathrm{C}_{4} \mathrm{H}_{9}\right]^{+}:$975.3124; found: 975.441 .

(1,8-bis(4-methoxyphenyl)pyrene-2,7-diyl)bis(tributylstannane) (compound 2d). Following

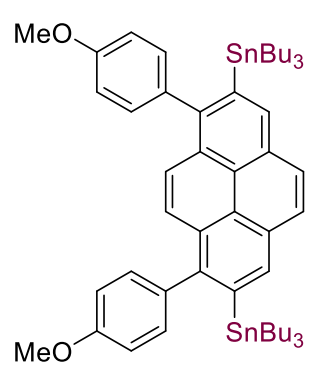
general procedure $B$, the title compound was synthesized from $0.3 \mathrm{~g}(0.63$ $\mathrm{mmol}$ ) of precursor $\mathbf{1 d}$. The product was isolated by column chromatography using $1 \%$ EtOAc in hexanes as eluent to give $0.214 \mathrm{~g}$ of the title compound as a clear oil (34\%).

${ }^{1} \mathrm{H}$ NMR $\left(400 \mathrm{MHz}, \mathrm{CDCl}_{3}, \delta\right.$ ): 8.29 (s accompanied with d peak with ${ }^{3} \mathrm{JH}_{\mathrm{H}-\mathrm{Sn}}=$ $42.4 \mathrm{~Hz}, 2 \mathrm{H}), 8.09(\mathrm{~s}, 2 \mathrm{H}), 7.65(\mathrm{~s}, 2 \mathrm{H}), 7.32(\mathrm{~d}, J=8.6 \mathrm{~Hz}, 4 \mathrm{H}), 7.02(\mathrm{~d}, J=8.6$ $\mathrm{Hz}, 4 \mathrm{H}), 3.91(\mathrm{~s}, 6 \mathrm{H}), 1.44-1.38(\mathrm{~m}, 12 \mathrm{H}), 1.33-1.24(\mathrm{~m}, 12 \mathrm{H}), 0.88-0.82(\mathrm{~m}$, $30 \mathrm{H})$.

${ }^{13} \mathrm{C}$ NMR (150 MHz, $\mathrm{CDCl}_{3}$, 8): 158.9, 144.7, 141.4, 136.0, 132.7, 131.8, 129.9, 127.1, 125.5, 113.4, $55.4,29.1,27.4,13.6,10.6$ (two sp² signals not observed due to overlapping).

MS (MALDI-TOF, positive, m/z): calcd. for $\mathrm{C}_{50} \mathrm{H}_{65} \mathrm{O}_{2} \mathrm{Sn}_{2}{ }^{+}\left[\mathrm{M}-\mathrm{C}_{4} \mathrm{H}_{9}\right]^{+}:$: 935.3023; found: 935.243. 
4,4'-(2,7-bis(tributylstannyl)pyrene-1,8-diyl)dibenzonitrile (compound 2e). Following general

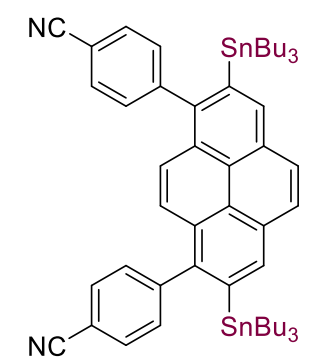
procedure $B$, the title compound was synthesized from $0.25 \mathrm{~g}(0.536 \mathrm{mmol})$ of precursor 1e. The product was isolated by column chromatography using $5 \%$ Et2O in hexanes as eluent to give $0.127 \mathrm{~g}$ of the title compound as a clear oil (24\%).

${ }^{1} \mathrm{H}$ NMR (400 MHz, $\left.\mathrm{CDCl}_{3}, 8\right): 8.35$ (s accompanied with d peak with ${ }^{3} \mathrm{H}_{\mathrm{H}-\mathrm{Sn}}=40.6$ $\mathrm{Hz}, 2 \mathrm{H}), 8.14(\mathrm{~s}, 2 \mathrm{H}), 7.81(\mathrm{~d}, J=7.7 \mathrm{~Hz}, 4 \mathrm{H}), 7.54(\mathrm{~d}, J=7.7 \mathrm{~Hz}, 4 \mathrm{H}), 7.48$ (s, $2 \mathrm{H}), 1.43-1.36(\mathrm{~m}, 12 \mathrm{H}), 1.32-1.23(\mathrm{~m}, 12 \mathrm{H}), 0.88-0.80(\mathrm{~m}, 30 \mathrm{H})$.

${ }^{13} \mathrm{C}$ NMR (150 MHz, $\left.\mathrm{CDCl}_{3}, \delta\right):$ 148.5, 142.9, 140.8, 133.3, 131.9, 131.6, 130.4, 128.0, 127.7, 125.2, 111.4, 29.0, 27.3, 13.6, 10.7 (two sp ${ }^{2}$ signals not observed due to overlapping).

MS (MALDI-TOF, positive, m/z): calcd. for $\mathrm{C}_{50} \mathrm{H}_{59} \mathrm{~N}_{2} \mathrm{Sn}_{2}{ }^{+}\left[\mathrm{M}-\mathrm{C}_{4} \mathrm{H}_{9}\right]^{+}$: 925.2716; found: 925.167.

\section{(1,8-bis(4-(tert-butyl)phenyl)benzo[e]pyrene-2,7-diyl)bis(tributylstannane) (compound 2g).}

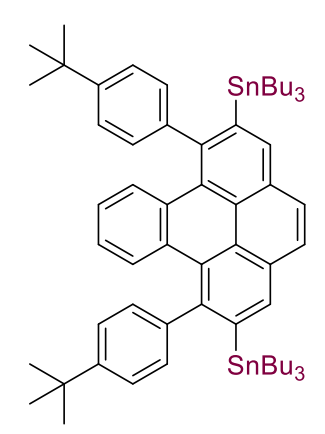

Following general procedure $B$, the title compound was synthesized from 0.35 $\mathrm{g}(0.604 \mathrm{mmol})$ of precursor $1 \mathrm{~g}$. The product was isolated by column chromatography using hexanes as eluent to give $0.113 \mathrm{~g}$ the title compound as a clear oil (17\%).

${ }^{1} \mathrm{H}$ NMR (400 MHz, $\mathrm{CDCl}_{3}, \delta$ ): 8.24 (s accompanied with d peak with ${ }^{3} \mathrm{JH}_{\mathrm{H}-\mathrm{Sn}}=$ $44.3 \mathrm{~Hz}, 2 \mathrm{H}), 7.98(\mathrm{~s}, 2 \mathrm{H}), 7.70-7.67(\mathrm{~m}, 2 \mathrm{H}), 7.47(\mathrm{~d}, J=8.3 \mathrm{~Hz}, 4 \mathrm{H}), 7.39$ (d, $J=8.3 \mathrm{~Hz}, 4 \mathrm{H}), 1.43-1.23(\mathrm{~m}, 42 \mathrm{H}), 0.88-0.76(\mathrm{~m}, 30 \mathrm{H})$.

${ }^{13} \mathrm{C} \mathrm{NMR}\left(150 \mathrm{MHz}, \mathrm{CDCl}_{3}, \delta\right): 150.1,145.0,144.2,144.1,134.8,132.6,130.1$,

129.7, 129.2, 127.9, 126.6, 126.1, 126.0, 124.4, 34.6, 31.5, 29.1, 27.3, 13.7, 11.1.

MS (MALDI-TOF, positive, m/z): calcd. for $\mathrm{C}_{60} \mathrm{H}_{79} \mathrm{Sn}_{2}{ }^{+}\left[\mathrm{M}-\mathrm{C}_{4} \mathrm{H}_{9}\right]^{+}: 1037.4215$; found: 1037.436.

(1,7-bis(4-(tert-butyl)phenyl)perylene-2,8-diyl)bis(tributylstannane) (compound $\mathbf{2 h}$ ). Following

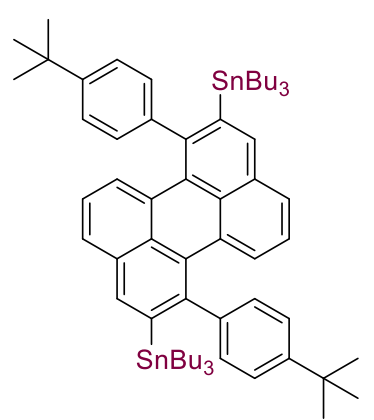
general procedure $B$, the title compound was synthesized from $0.25 \mathrm{~g}$ ( $0.432 \mathrm{mmol}$ ) of precursor $\mathbf{1 h}$. The product was isolated by column chromatography using $1 \% \mathrm{Et} 2 \mathrm{O}$ in hexanes as eluent to give $0.077 \mathrm{~g}$ of the title compound as a green oil (16\%).

${ }^{1} \mathrm{H}$ NMR (400 MHz, $\mathrm{CDCl}_{3}, \delta$ ): 7.81 (s accompanied with d peak with ${ }^{3} \mathrm{H}_{\mathrm{H}-\mathrm{Sn}}$ $=46.6 \mathrm{~Hz}, 2 \mathrm{H}), 7.48(\mathrm{~d}, J=7.8 \mathrm{~Hz}, 2 \mathrm{H}), 7.41(\mathrm{~d}, J=8.2 \mathrm{~Hz}, 4 \mathrm{H}), 7.28(\mathrm{~d}, J$ $=8.2 \mathrm{~Hz}, 4 \mathrm{H}), 7.15(\mathrm{~d}, J=7.8 \mathrm{~Hz}, 2 \mathrm{H}), 6.93(\mathrm{t}, J=7.8 \mathrm{~Hz}, 2 \mathrm{H}), 1.39-1.23$ $(\mathrm{m}, 42 \mathrm{H}), 0.87-0.69(\mathrm{~m}, 30 \mathrm{H})$.

$\left.{ }^{13} \mathrm{C} \mathrm{NMR} \mathrm{(150} \mathrm{MHz,} \mathrm{CDCl}_{3}, \delta\right): 150.2,144.3,144.3,144.2,136.5,132.0,131.6,132.2,129.7,128.9$, $128.3,126.2,125.7,124.8,34.6,31.4,29.1,27.3,13.6,10.9$.

MS (MALDI-TOF, positive, m/z): calcd. for $\mathrm{C}_{60} \mathrm{H}_{79} \mathrm{Sn}_{2}{ }^{+}\left[\mathrm{M}-\mathrm{C}_{4} \mathrm{H}_{9}\right]^{+}: 1037.4215$; found: 1037.433 . 
(1,10-diphenyldibenzo[cd,Im] perylene-2,9-diyl)bis(tributylstannane) (compound 2i). Following

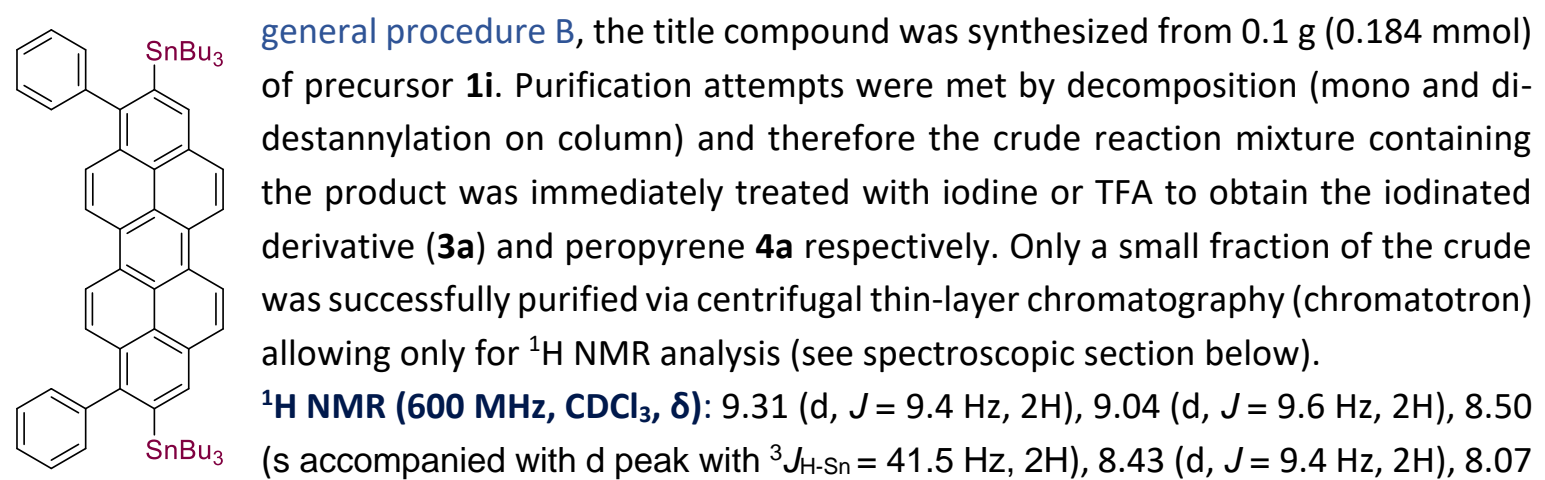

(d, $J=9.6 \mathrm{~Hz}, 2 \mathrm{H}), 7.58-7.51(\mathrm{~m}, 10 \mathrm{H}), 1.46-0.83$ ("grease" overlapping with butyl signals from $\mathrm{Bu}_{3} \mathrm{Sn}$ made analysis impossible at this scale; theoretical $=72 \mathrm{H}$ ).

MS (MALDI-TOF, positive, m/z): calcd. for $\mathrm{C}_{58} \mathrm{H}_{65} \mathrm{Sn}_{2}{ }^{+}\left[\mathrm{M}-\mathrm{C}_{4} \mathrm{H}_{9}\right]^{+}$: 999.3119; found: 999.550 .

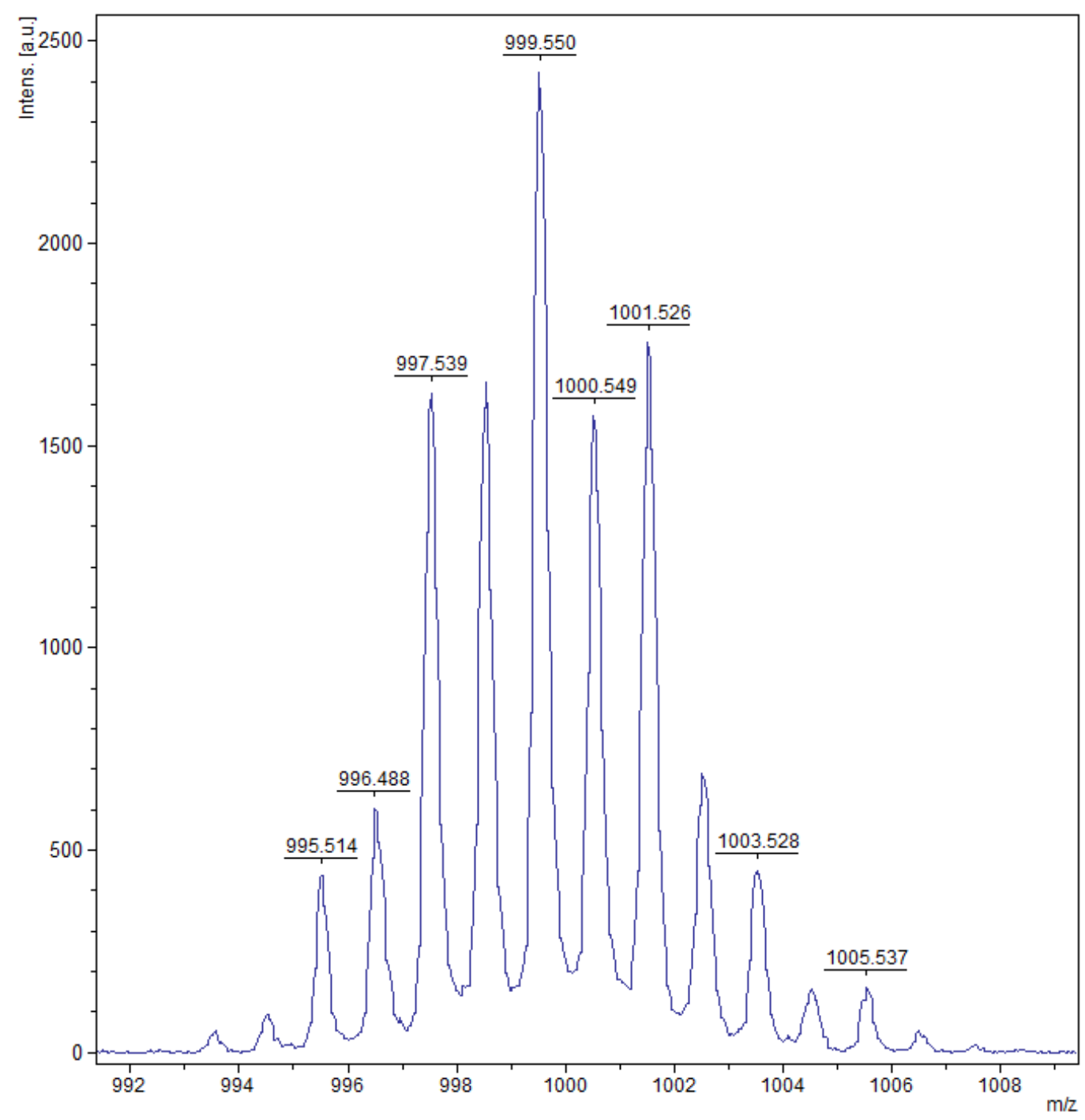

Figure S8. MALDI mass spectrum of $\mathbf{2} \mathbf{i}$ as an illustration of the isotopic distribution of the distannylarene series. 
Synthesis of (3,6-diphenylbenzo[e]pyrene-2,7-diyl)bis(tributylstannane) and (3,9diphenylperylene-2,8-diyl)bis(tributylstannane) (compounds $2 f$ and $2 f^{\prime}$ )

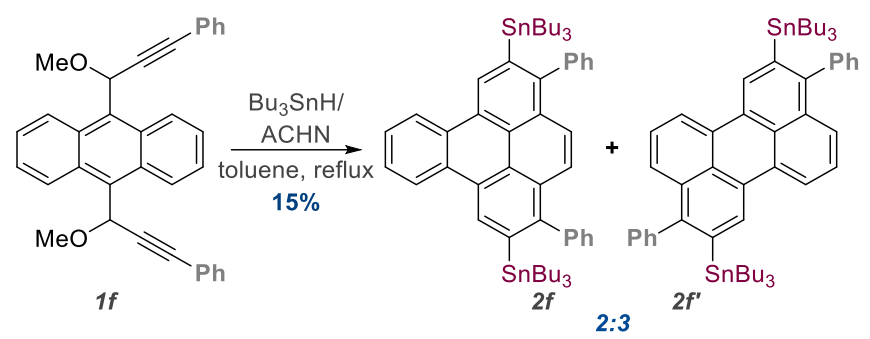

Following general procedure $B$, the isomeric mixture was prepared from $0.16 \mathrm{~g}(0.343 \mathrm{mmol})$ of precursor 1f. ${ }^{1} \mathrm{H}$ NMR spectra of the crude reaction mixture was recorded to identify the ratio of products $\mathbf{2} \mathbf{f}$ and $\mathbf{2} \mathbf{f}^{\prime}$ (vide infra). A measured amount of dibromomethane was added as an internal standard to calculate the combined yield. Attempts to isolate and separate the product mixture were performed by column chromatography using 3\% EtOAc in hexanes as eluent to give coelution of product $\mathbf{2} \mathbf{f}$ and $\mathbf{2} \mathbf{f}^{\prime}$ with different ratios per fraction. From an 'enriched' fraction of $\mathbf{2} \mathbf{f}^{\prime}$ ( $\sim$ 4:1 ratio of $\mathbf{2} \mathbf{f}^{\prime}$ to $\mathbf{2} \mathbf{f}$ ), crystals of $\mathbf{2} \mathbf{f}^{\prime}$ ' were grown (see crystallographic section) and identification of key $1 \mathrm{H}$ NMR signals for ratio determination were assigned.

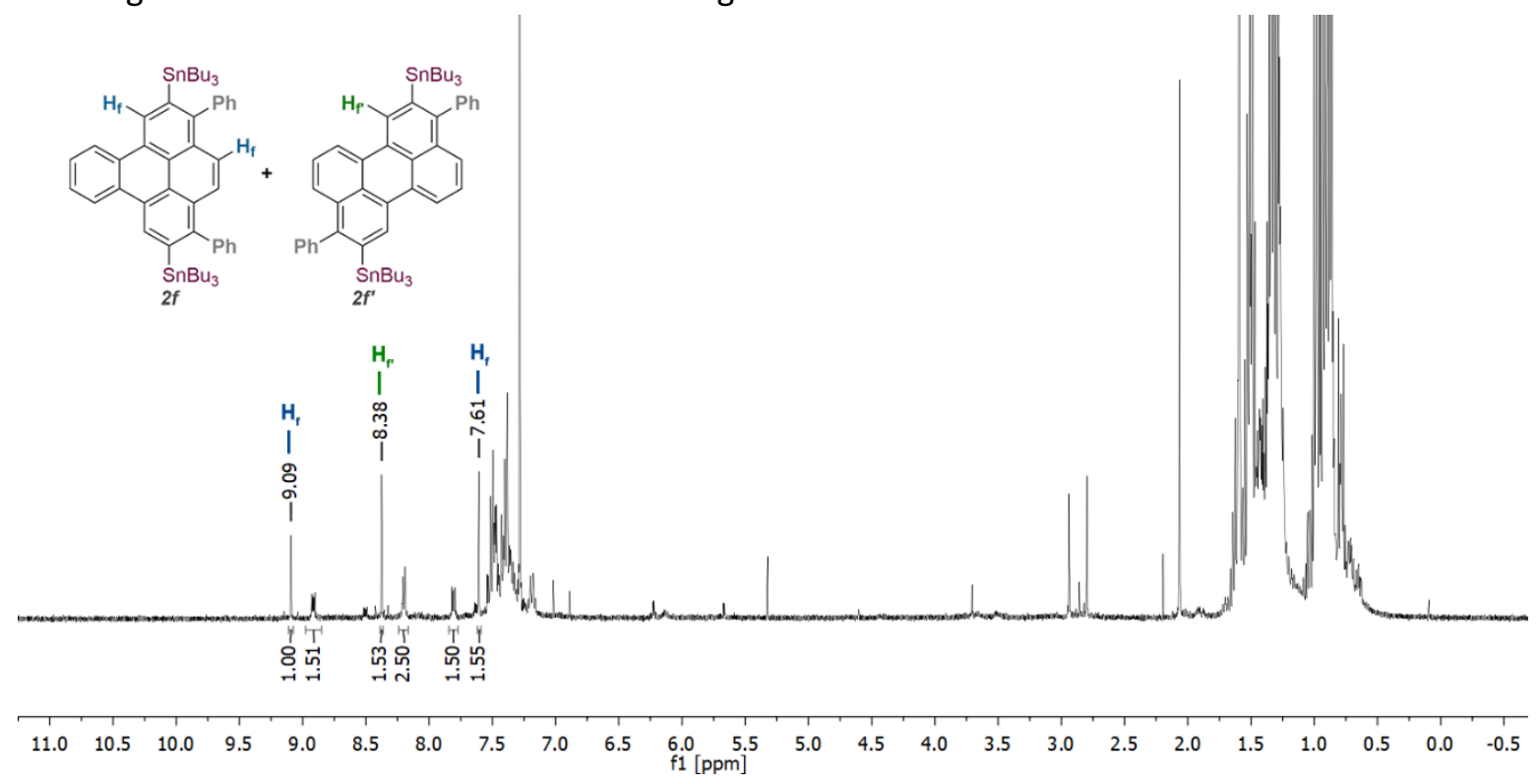

Figure S9. ${ }^{1} \mathrm{H} N M R\left(400 \mathrm{MHz}, \mathrm{CDCl}_{3}\right)$ of crude reaction mixture $\mathbf{2 f} / \mathbf{2} \mathbf{f}^{\prime}$. The $\delta=7.61 \mathrm{ppm}$ signal for $\mathbf{2 f}$ (proton not adjacent to $\mathrm{Sn}$ due to lack of satellites) has an overlapping signal which increases its integration. 


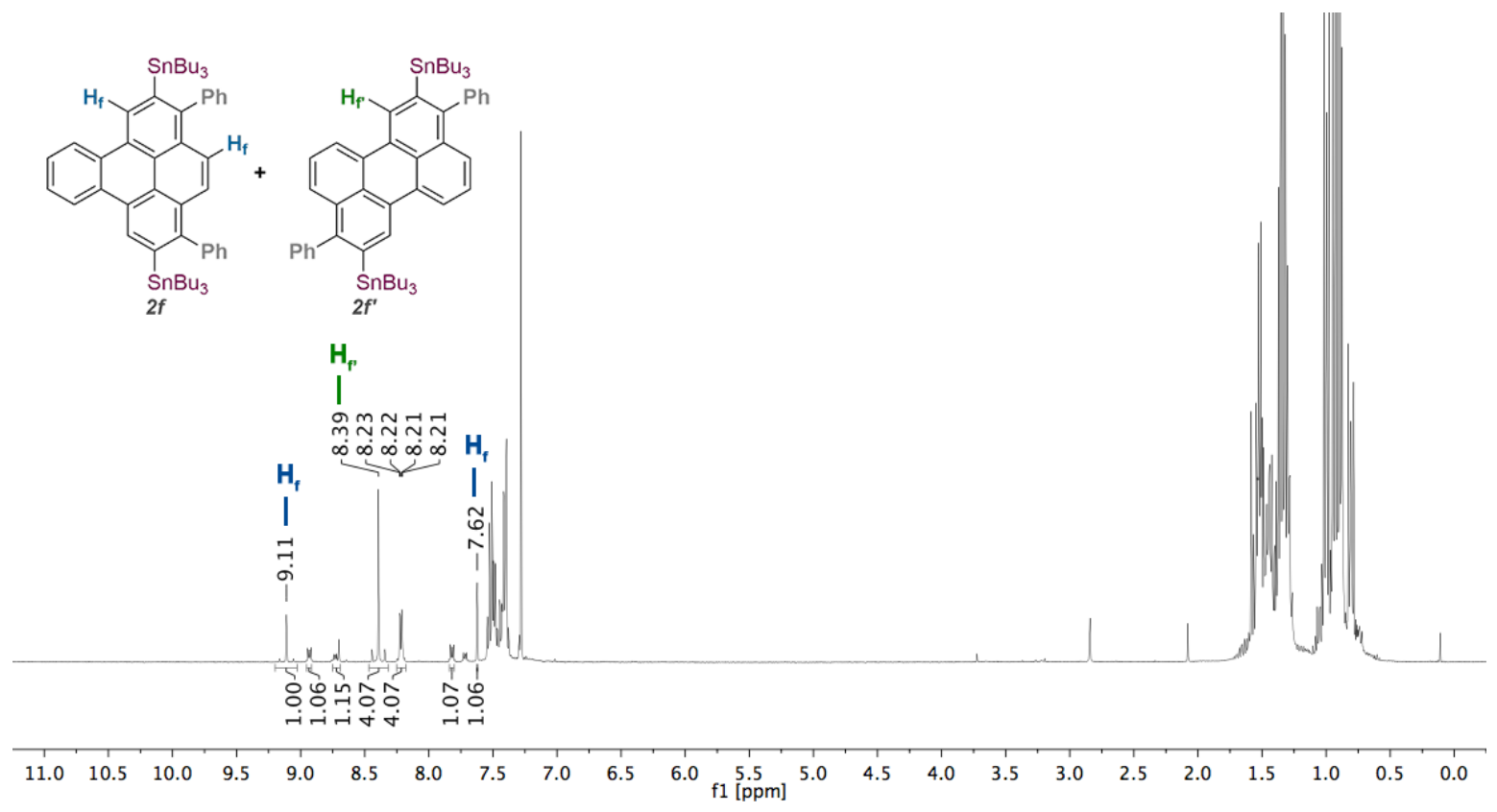

Figure S10. ${ }^{1} \mathrm{H}$ NMR $\left(400 \mathrm{MHz}, \mathrm{CDCl}_{3}\right)$ of enriched $\mathbf{2} \mathbf{f}^{\prime}$ fraction.
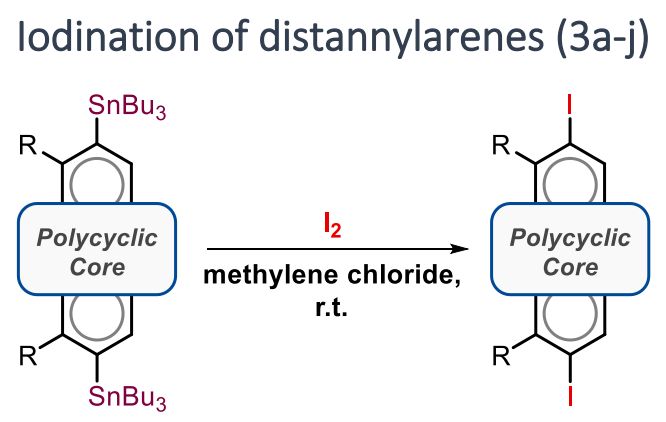

General procedure C:

To a stirring solution of distannylarene (2a-j) in methylene chloride were added $I_{2}$ crystals ( 2.2 equiv.) under ambient atmosphere. Addition of $\mathrm{I}_{2}$ was stopped when a dark color for the solution persisted-indicating full consumption of starting material. The reaction mixture was stirred at room temperature for 15 minutes and then quenched with a saturated aqueous solution of $\mathrm{Na}_{2} \mathrm{~S}_{2} \mathrm{O}_{3}$. The biphasic mixture was left stirring vigorously for 30 minutes. Then, the mixture was extracted with methylene chloride (x3). The combined organic phase was dried over $\mathrm{MgSO}_{4}$ and concentrated under vacuum. Purifications were performed through column chromatography and/or recrystallization. 
1,8-dibutyl-2,7-diiodopyrene (compound 3a). Following general procedure $C$, the title compound<smiles>CCCCc1c(I)cc2ccc3cc(I)c(CCCC)c4ccc1c2c34</smiles>
was synthesized from $0.1 \mathrm{~g}(0.112 \mathrm{mmol})$ of $2 \mathrm{a}$. The product was isolated by recrystallization from hexanes to give $0.052 \mathrm{~g}$ of the title product as a white solid (92\%).

${ }^{1} \mathrm{H}$ NMR $\left(400 \mathrm{MHz} \mathrm{CDCl}_{3}, \delta\right): 8.50(\mathrm{~s}, 2 \mathrm{H}), 8.15(\mathrm{~s}, 2 \mathrm{H}), 7.66(\mathrm{~s}, 2 \mathrm{H}), 3.44-3.40$ (m, 4H), 1.74-1.58 (m, $8 \mathrm{H}), 1.07(\mathrm{t}, J=7.2 \mathrm{~Hz}, 6 \mathrm{H})$.

${ }^{13} \mathrm{C}$ NMR (150 MHz, $\left.\mathrm{CDCl}_{3}, \delta\right): 139.1,135.5,131.0,128.4,126.2,124.8,124.6$, $100.5,37.8,32.7,23.1,14.0$.

HRMS (DART, positive, $\mathrm{m} / \mathrm{z}$ ): calcd. for $\mathrm{C}_{24} \mathrm{H}_{25} \mathrm{I}_{2}{ }^{+}[\mathrm{M}+\mathrm{H}]^{+}: 567.0045$; found: 567.0066 .

2,7-diiodo-1,8-diphenylpyrene (compound 3b). Following general procedure $C$, the title compound<smiles>Ic1cc2ccc3cc(I)c(-c4ccccc4)c4ccc(c1-c1ccccc1)c2c34</smiles>
was synthesized from $0.108 \mathrm{~g}(0.1158 \mathrm{mmol})$ of $\mathbf{2} \mathbf{b}$. The product was isolated by recrystallization from hexanes to give $0.052 \mathrm{~g}$ of the title product as a white solid (>95\%).

${ }^{1} \mathrm{H}$ NMR (400 MHz, $\left.\mathrm{CDCl}_{3}, \delta\right): 8.73(\mathrm{~s}, 2 \mathrm{H}), 7.95(\mathrm{~s}, 2 \mathrm{H}), 7.52(\mathrm{~b}, 8 \mathrm{H}), 7.31-7.30(\mathrm{~m}$, $4 \mathrm{H})$.

${ }^{13} \mathrm{C}$ NMR (150 MHz, $\left.\mathrm{CDCl}_{3}, \delta\right): 143.1,141.8,135.0,132.1,130.3,129.7,128.3$, 128.0, 127.2, 127.1, 123.9, 99.3.

HRMS (DART, positive, $\mathrm{m} / \mathrm{z}$ ): calcd. for $\mathrm{C}_{28} \mathrm{H}_{16} \mathrm{I}_{2}^{+\bullet}[\mathrm{M}]^{+\bullet}: 605.9341$; found: 605.9345 .

(2,7-diiodo-1,8-di(naphthalen-1-yl)pyrene (compound 3c). Following general procedure $C$, the title

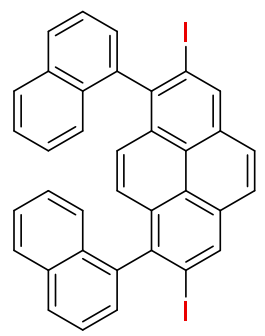
compound was synthesized from $0.56 \mathrm{~g}(0.0542 \mathrm{mmol})$ of $\mathbf{2 c}$. The product was isolated by recrystallization from methylene chloride/hexanes to give $0.036 \mathrm{~g}$ of the title product as a white solid (93\%).

${ }^{1} \mathrm{H}$ NMR (400 MHz, $\left.\mathrm{CDCl}_{3}, \delta\right): 8.87(\mathrm{~s}, 2 \mathrm{H}), 8.12(\mathrm{~s}, 2 \mathrm{H}), 7.97-7.91(\mathrm{~m}, 4 \mathrm{H}), 7.61-$ $7.56(\mathrm{~m}, 2 \mathrm{H}), 7.48-7.42(\mathrm{~m}, 2 \mathrm{H}), 7.39-7.36(\mathrm{~m}, 2 \mathrm{H}), 7.28-7.22(\mathrm{~m}, 2 \mathrm{H}), 7.12-7.09$ (m, 2H).

${ }^{13} \mathrm{C}$ NMR (150 MHz, $\left.\mathrm{CDCl}_{3}, \delta\right): 140.7,140.6,140.2,140.2,135.1,135.1,133.5$, $132.4,132.4,132.2,132.1,137.0,137.0,128.5,128.3,128.3,127.5,127.5,127.3,126.4,126.4$, $126.0,125.8,125.7,125.3,123.9,100.4,100.3$. ("extra" signals found caused by mixture of atropisomers).

HRMS (DART, positive, m/z): calcd. for $\mathrm{C}_{36} \mathrm{H}_{20} \mathrm{I}_{2}{ }^{+\bullet}[\mathrm{M}]^{+\bullet}:$ 705.9654; found: 705.9656.

2,7-diiodo-1,8-bis(4-methoxyphenyl)pyrene (compound 3d). Following general procedure $C$, the<smiles>COc1ccc(-c2c(I)cc3ccc4cc(I)c(-c5ccc(OC)cc5)c5ccc2c3c45)cc1</smiles>
title compound was synthesized from $0.065 \mathrm{~g}(0.0655 \mathrm{mmol})$ of $\mathbf{2 d}$. The product was isolated by recrystallization from methylene chloride/hexanes to give $0.042 \mathrm{~g}$ of the title product as a white solid (>95\%).

${ }^{1} \mathrm{H}$ NMR (400 MHz, $\left.\mathrm{CDCl}_{3}, \delta\right): 8.75(\mathrm{~s}, 2 \mathrm{H}), 7.98(\mathrm{~s}, 2 \mathrm{H}), 7.57(\mathrm{~s}, 2 \mathrm{H}), 7.23$ (d, J $=8.6 \mathrm{~Hz}, 4 \mathrm{H}), 7.05(\mathrm{~d}, J=8.6 \mathrm{~Hz}, 4 \mathrm{H}), 3.91(\mathrm{~s}, 6 \mathrm{H})$.

${ }^{13} \mathrm{C} \mathrm{NMR}\left(150 \mathrm{MHz}, \mathrm{CDCl}_{3}, \delta\right): 159.3,141.5,135.5,134.8,132.0,131.5,130.1$, 127.3, 127.0, 124.0, 100.3, 55.3.

HRMS (DART, positive, $\mathrm{m} / \mathrm{z}$ ): calcd. for $\mathrm{C}_{30} \mathrm{H}_{20} \mathrm{O}_{2} \mathrm{O}_{2}^{+\bullet}[\mathrm{M}]^{+\bullet}: 665.9552$; found: 665.9573 . 
4,4'-(2,7-diiodopyrene-1,8-diyl)dibenzonitrile (compound 3e). Following general procedure $C$, the<smiles>N#Cc1ccc(-c2c(I)cc3ccc4cc(I)c(-c5ccc(C#N)cc5)c5ccc2c3c45)cc1</smiles>
title compound was synthesized from $0.1 \mathrm{~g}(0.0102 \mathrm{mmol})$ of $2 \mathrm{e}$. The product was isolated by running a silica plug with toluene as eluent followed by recrystallization from methylene chloride/hexanes to give $0.061 \mathrm{~g}$ of the title product as a white solid (91\%).

${ }^{1} \mathrm{H}$ NMR $\left(400 \mathrm{MHz}, \mathrm{CDCl}_{3}, \delta\right): 8.81(\mathrm{~s}, 2 \mathrm{H}), 8.06(\mathrm{~s}, 2 \mathrm{H}), 7.85$ (d, $\left.J=7.8 \mathrm{~Hz}, 4 \mathrm{H}\right)$, $7.46(\mathrm{~d}, J=7.8 \mathrm{~Hz}, 4 \mathrm{H}), 7.42(\mathrm{~s}, 2 \mathrm{H})$.

${ }^{13} \mathrm{C}$ NMR (150 MHz, $\left.\mathrm{CDCl}_{3}, \delta\right): 147.5,139.9,135.5,132.6,132.3,131.4,129.2$, 127.6, 126.9, 123.7, 118.6, 112.3, 98.1 .

MS (MALDI-TOF, positive, m/z): calcd. for $\mathrm{C}_{30} \mathrm{H}_{14} \mathrm{I}_{2} \mathrm{~N}_{2}{ }^{+}[\mathrm{M}]^{+}$: 655.9246; found: 655.839 .

1,8-bis(4-(tert-butyl)phenyl)-2,7-diiodobenzo[e]pyrene (compound $\mathbf{3 g}$ ). Following general<smiles></smiles>
procedure $C$, the title compound was synthesized from $0.08 \mathrm{~g}(0.07307 \mathrm{mmol})$ of $\mathbf{2 g}$. The product was isolated by preparative thin layer chromatography using $7 \% \mathrm{Et}_{2} \mathrm{O}$ in hexanes as eluent to give $0.051 \mathrm{~g}$ of the title product as an off-white solid (89\%).

${ }^{1} \mathrm{H}$ NMR $\left(400 \mathrm{MHz}, \mathrm{CDCl}_{3}, \delta\right): 8.77(\mathrm{~s}, 2 \mathrm{H}), 7.87(\mathrm{~s}, 2 \mathrm{H}), 7.64-7.62(\mathrm{~m}, 2 \mathrm{H}), 7.51$ (d, $J=8.2 \mathrm{~Hz}, 4 \mathrm{H}), 7.35(\mathrm{~d}, J=8.2 \mathrm{~Hz}, 4 \mathrm{H}), 6.83-6.81(\mathrm{~m}, 2 \mathrm{H}), 1.41(\mathrm{~m}, 18 \mathrm{H})$. ${ }^{13} \mathrm{C} \mathrm{NMR}\left(150 \mathrm{MHz}, \mathrm{CDCl}_{3}, \delta\right): 151.0,143.6,140.2,137.2,131.7,131.6,130.8$, 130.0, 129.6, 126.5, 125.7, 125.6, 125.3, 103.4, 34.7, 31.4.

HRMS (DART, positive, $\mathrm{m} / \mathrm{z}$ ): calcd. for $\mathrm{C}_{40} \mathrm{H}_{34} \mathrm{I}_{2}^{+\bullet}[\mathrm{M}]^{+\bullet}:$ 768.0750; found: 768.0751 .

2,9-diiodo-1,10-diphenyldibenzo[cd,Im]perylene (compound 3i). Following general procedure C,

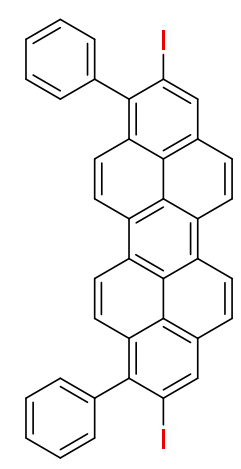
the title compound was synthesized from crude reaction mixture $\mathbf{2} \mathbf{i}$. The product was isolated by silica plug followed by recrystallization from methylene chloride/hexanes to give $0.043 \mathrm{~g}$ of the title product as a yellow solid ( $83 \%$ from $0.0710 \mathrm{mmol} 2 \mathrm{i}$ calculated in crude).

${ }^{1} \mathrm{H} \mathrm{NMR}\left(400 \mathrm{MHz}, \mathrm{CDCl}_{3}, \delta\right): 9.22(\mathrm{~d}, J=9.3 \mathrm{~Hz}, 2 \mathrm{H}), 8.99$ (d, $\left.J=9.5 \mathrm{~Hz}, 2 \mathrm{H}\right), 8.90$ (s, 2H), 8.29 (d, $J=9.3 \mathrm{~Hz}, 2 \mathrm{H}), 7.98(\mathrm{~d}, J=9.5 \mathrm{~Hz}, 2 \mathrm{H}), 7.65-7.59(\mathrm{~m}, 6 \mathrm{H}), 7.46-$ $7.44(\mathrm{~m}, 4 \mathrm{H})$.

${ }^{13} \mathrm{C}$ NMR (150 MHz, $\left.\mathrm{CDCl}_{3}, \delta\right): 143.5,141.6,134.7,132.2,130.6,130.5,128.4$, 128.09, 127.0, 126.7, 125.8, 125.0, 124.6, 123.7, 123.4, 122.9, 99.3.

MS (MALDI-TOF, positive, m/z): calcd. for $\mathrm{C}_{38} \mathrm{H}_{20} \mathrm{O}_{2}{ }^{+}[\mathrm{M}]^{+}:$: 729.9654; found: 729.904 . 
1,8-bis(4-(tert-butyl)phenyl)-2,7-diiodopyrene (compound 3j). The title compound was<smiles>CC(C)(C)c1ccc(-c2c(I)cc3ccc4cc(I)c(-c5ccc(C(C)(C)C)cc5)c5ccc2c3c45)cc1</smiles>
synthesized via one-pot cyclization/iodination (general procedure B and C) from $1.0 \mathrm{~g}(1.8913 \mathrm{mmol})$ of precursor $\mathbf{1 j}$. The product was isolated by column chromatography using hexanes as eluent to give $0.463 \mathrm{~g}$ of the title product (32\% from 1j).

${ }^{1} \mathrm{H}$ NMR $\left(400 \mathrm{MHz} \mathrm{CDCl}_{3}, \delta\right): 8.75(\mathrm{~s}, 2 \mathrm{H}), 7.99(\mathrm{~s}, 2 \mathrm{H}), 7.55-7.50(\mathrm{~m}, 6 \mathrm{H}), 7.23$ (d, $J=7.9 \mathrm{~Hz}, 4 \mathrm{H}) 1.40(\mathrm{~s}, 18 \mathrm{H})$.

${ }^{13} \mathrm{C}$ NMR (150 MHz, $\left.\mathrm{CDCl}_{3}, \delta\right): 150.9,142.1,140.2,135.0,132.2,130.1,130.0$, 127.4, 127.1, 125.2, 124.1, 99.7, 34.8, 31.6.

HRMS (DART, positive, $\mathrm{m} / \mathrm{z}$ ): calcd. for $\mathrm{C}_{36} \mathrm{H}_{32} \mathrm{I}_{2}^{+\bullet}[\mathrm{M}]^{+\bullet}:$ 718.0593; found: 718.0590 .

lodination of distannylarene $2 f^{\prime}$ (compound $3 f^{\prime}$ )

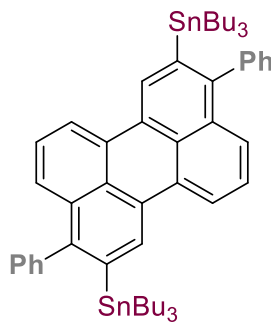

$2 f$ ' 'enriched'

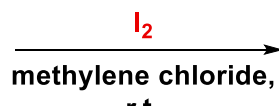
r.t.<smiles></smiles>

Compound 3f' was prepared by iodination of an 'enriched' fraction $\mathbf{2 f}$ ' ( $\sim$ :1 ratio of $\mathbf{2} \mathbf{f}^{\prime}$ to $\mathbf{2 f}$ ) following general procedure C. 3f' was isolated by centrifugal thin-layer chromatography (chromatotron) and crystals were grown by slow evaporation from $\mathrm{Et}_{2} \mathrm{O}$ (see crystallographic section).

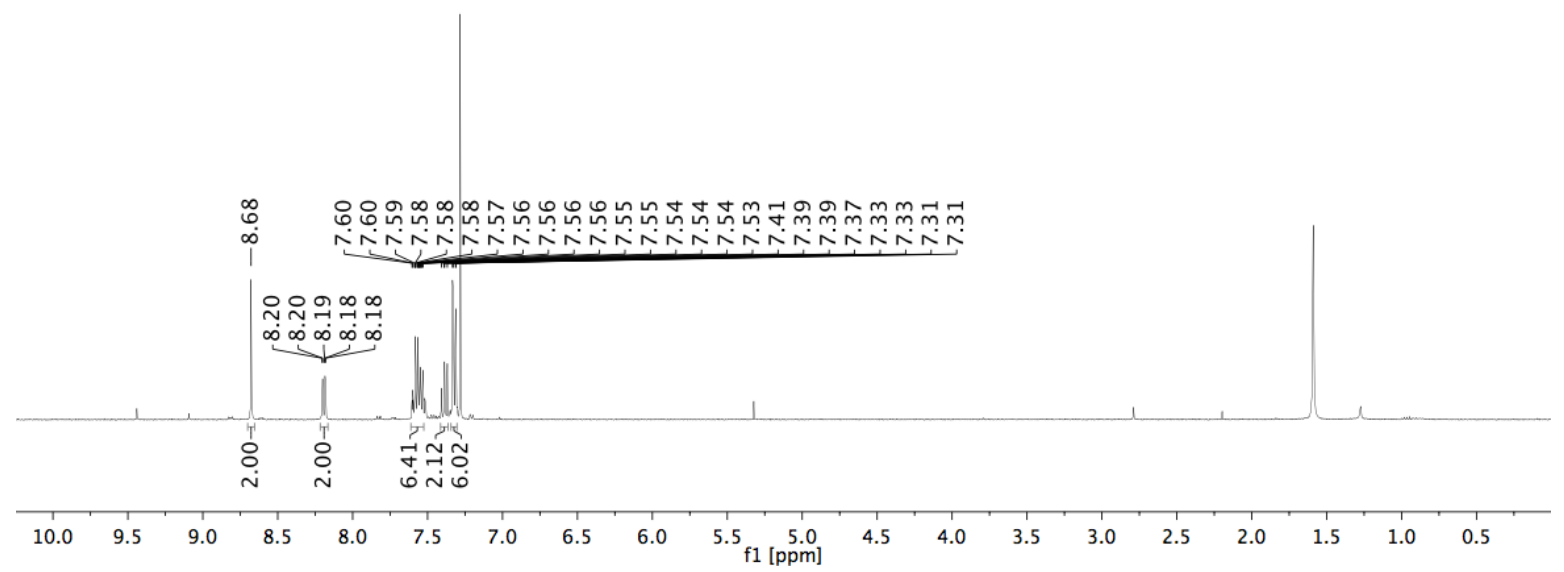

Figure S11. ${ }^{1} \mathrm{H} \mathrm{NMR}\left(400 \mathrm{MHz}, \mathrm{CDCl}\right.$ ) of compound $\mathbf{3 f}^{\prime}$. 
Protodestannylation of distannylarenes (compounds 4a-i)

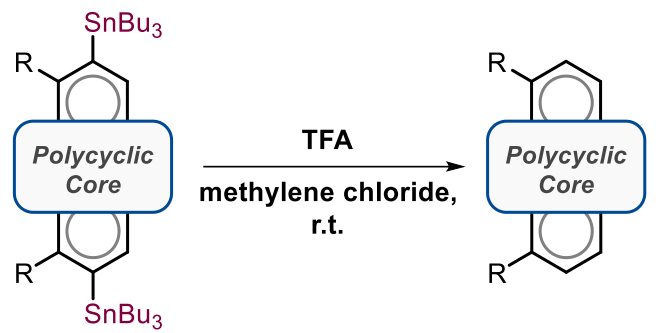

General procedure D:

To a stirring solution of distannylarene (2a-j) in methylene chloride was added TFA (or $2 \mathrm{M} \mathrm{HCl} ; 5.0$ equiv.) dropwise under ambient atmosphere. The reaction mixture was stirred at room temperature for 15 minutes and then quenched by slowly adding a saturated solution of $\mathrm{NaHCO}_{3}$ until bubbles no longer evolved $(\mathrm{pH} \sim 9)$. The biphasic mixture was left stirring vigorously for 30 minutes. The mixture was then transferred to a separatory funnel where the aqueous phase was extracted with methylene chloride (x3). The combined organic phase was washed with brine, dried over $\mathrm{MgSO}_{4}$ and concentrated under vacuum. Purifications were performed through column chromatography and/or recrystallization.

1,8-dibutylpyrene (compound 4a). Following general procedure $D$, the title compound was

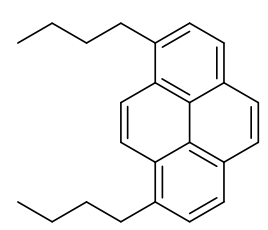
synthesized from $0.1 \mathrm{~g}(0.112 \mathrm{mmol})$ of $\mathbf{2 a}$. The product was isolated by column chromatography using hexanes as eluent to give $0.034 \mathrm{~g}$ of the title product as an off-white solid (>95\%).

${ }^{1} \mathrm{H}$ NMR (400 MHz, $\left.\mathrm{CDCl}_{3}, \delta\right): 8.31(\mathrm{~s}, 2 \mathrm{H}), 8.07(\mathrm{~d}, J=7.7 \mathrm{~Hz}, 2 \mathrm{H}), 7.96(\mathrm{~s}, 2 \mathrm{H})$, 7.85 (d, $J=7.7 \mathrm{~Hz}, 2 \mathrm{H}$ ), 3.34 (t, $J=7.8 \mathrm{~Hz}, 4 \mathrm{H}$ ), 1.85 (quin, $J=7.8 \mathrm{~Hz} 4 \mathrm{H}$ ), 1.45 (sext, $J=7.3 \mathrm{~Hz}, 4 \mathrm{H}$ ), 1.01 (t, $J=7.3 \mathrm{~Hz}, 6 \mathrm{H}$ ).

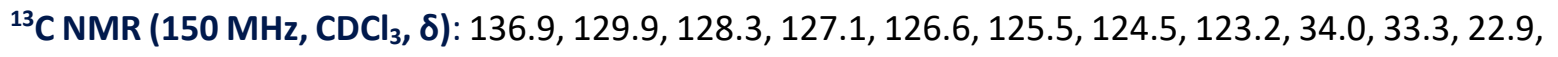
14.0.

HRMS (DART, positive, $\mathrm{m} / \mathrm{z}$ ): calcd. for $\mathrm{C}_{24} \mathrm{H}_{27}{ }^{+}[\mathrm{M}+\mathrm{H}]^{+}:$315.2113; found: 315.2133 .

1,8-diphenylpyrene (compound $4 \mathbf{b}$ ). Following general procedure $D$, the title compound was<smiles>c1ccc(-c2ccc3ccc4c(-c5ccccc5)ccc5ccc2c3c54)cc1</smiles>
synthesized from $0.1 \mathrm{~g}(0.1072 \mathrm{mmol})$ of $\mathbf{2 b}$. The product was isolated by column chromatography using 1\% EtOAc in Hexanes as eluent to give $0.037 \mathrm{~g}$ of the title product as pale-yellow needles (>95\%).

${ }^{1} \mathrm{H}$ NMR (400 MHz, $\left.\mathrm{CDCl}_{3}, \delta\right): 8.25(\mathrm{~d}, J=7.8 \mathrm{~Hz}, 2 \mathrm{H}), 8.15(\mathrm{~s}, 2 \mathrm{H}), 8.13(\mathrm{~s}, 2 \mathrm{H})$, $8.01(\mathrm{~d}, J=7.8 \mathrm{~Hz}, 2 \mathrm{H}), 7.66-7.64(\mathrm{~m}, 4 \mathrm{H}), 7.57-7.54(\mathrm{~m}, 4 \mathrm{H}), 7.50-7.46(\mathrm{~m}, 2 \mathrm{H})$.

${ }^{13} \mathrm{C}$ NMR (150 MHz, $\left.\mathrm{CDCl}_{3}, \delta\right): 141.1,137.6,130.9,130.6,128.3,127.7,127.4$, $127.2,125.4,125.2,124.8$.

HRMS (DART, positive, m/z): calcd. for $\mathrm{C}_{28} \mathrm{H}_{19}{ }^{+}[\mathrm{M}+\mathrm{H}]^{+}:$355.1487; found: 355.1502 . 
(1,8-di(naphthalen-1-yl)pyrene (compound 4c). Following general procedure $D$, the title compound

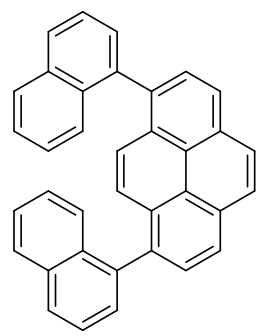
was synthesized from $0.07 \mathrm{~g}(0.0678 \mathrm{mmol})$ of $\mathbf{2 c}$. The product was isolated by recrystallization from methylene chloride/hexanes to give $0.029 \mathrm{~g}$ of the title product as an off-white solid ( $>95 \%)$.

${ }^{1} \mathrm{H}$ NMR $\left(400 \mathrm{MHz} \mathrm{CDCl}_{3}, \delta\right): 8.33(\mathrm{~d}, J=7.8 \mathrm{~Hz}, 2 \mathrm{H}), 8.24(\mathrm{~s}, 2 \mathrm{H}), 8.05(\mathrm{~d}, J=7.8$ $\mathrm{Hz}, 2 \mathrm{H}), 7.93(\mathrm{t}, J=7.1,4 \mathrm{H}), 7.61-7.4(\mathrm{~m}, 4 \mathrm{H}), 7.50(\mathrm{~s}, 2 \mathrm{H}) 7.46-7.40(\mathrm{~m}, 4 \mathrm{H})$, 7.26-7.23 (m, 2H).

${ }^{13} \mathrm{C}$ NMR (150 MHz, $\left.\mathrm{CDCl}_{3}, \delta\right): 138.7,138.6,136.0,135.9,133.5,133.5,132.9$, $131.1,131.1,129.7,129.7,128.5,128.5,128.3,128.3,128.1,127.9,127.5,126.5,126.0,125.8$, $125.7,125.7,125.3,125.2,124.9,124.8,124.7,124.6$. ("extra" signals found caused by mixture of atropisomers).

HRMS (DART, positive, $\mathrm{m} / \mathrm{z}$ ): calcd. for $\mathrm{C}_{36} \mathrm{H}_{22}{ }^{+\bullet}[\mathrm{M}]^{+\bullet}: 454.1721$; found: 454.1722 .

1,8-bis(4-methoxyphenyl)pyrene (compound 4d). Following general procedure $D$, the title<smiles>COc1ccc(-c2ccc3ccc4ccc(-c5ccc(OC)cc5)c5ccc2c3c45)cc1</smiles>
compound was synthesized from $0.1 \mathrm{~g}(0.1007 \mathrm{mmol})$ of $\mathbf{2 d}$. The product was isolated by recrystallization from methylene chloride/hexanes giving $0.04 \mathrm{~g}$ of the title product as a pale-yellow solid (>95\%).

${ }^{1} \mathrm{H}$ NMR $\left(400 \mathrm{MHz}, \mathrm{CDCl}_{3}, \delta\right): 8.21(\mathrm{~d}, J=7.8 \mathrm{~Hz}, 2 \mathrm{H}), 8.14(\mathrm{~s}, 2 \mathrm{H}), 8.10$ (s, $2 \mathrm{H}), 7.97(\mathrm{~d}, 7.8 \mathrm{~Hz}, 2 \mathrm{H}), 7.56(\mathrm{~d}, 8.6 \mathrm{~Hz}, 4 \mathrm{H}), 7.09(\mathrm{~d}, 8.6 \mathrm{~Hz}, 4 \mathrm{H}), 3.92(\mathrm{~s}, 6 \mathrm{H})$. ${ }^{13} \mathrm{C} \mathrm{NMR}\left(150 \mathrm{MHz}, \mathrm{CDCl}_{3}, \delta\right): 159.0,137.2,133.5,131.6,130.7,128.4,127.7$, $127.3,125.3,124.7,113.8,113.5,55.4$.

HRMS (DART, positive, $\mathrm{m} / \mathrm{z}$ ): calcd. for $\mathrm{C}_{30} \mathrm{H}_{22} \mathrm{O}_{2}{ }^{+}[\mathrm{M}]^{+\bullet}: 414.1619$; found: 414.1632 .

4,4'-(pyrene-1,8-diyl)dibenzonitrile (compound 4e). Following general procedure $D$, the title<smiles>N#Cc1ccc(-c2ccc3ccc4ccc(-c5ccc(C#N)cc5)c5ccc2c3c45)cc1</smiles>
compound was synthesized from $0.05 \mathrm{~g}(0.0509 \mathrm{mmol})$ of $2 \mathrm{e}$. The product was isolated by running a silica plug with toluene as eluent followed by recrystallization from methylene chloride/hexanes giving $0.02 \mathrm{~g}$ of the title product as a yellow solid (>95\%).

${ }^{1} \mathrm{H} \mathrm{NMR}\left(400 \mathrm{MHz} \mathrm{CDCl}_{3}, \delta\right): 8.33(\mathrm{~d}, J=7.8 \mathrm{~Hz}, 2 \mathrm{H}), 8.20(\mathrm{~s}, 2 \mathrm{H}), 8.07(\mathrm{~s}, 2 \mathrm{H})$, $7.01(\mathrm{~d}, 7.8 \mathrm{~Hz}, 2 \mathrm{H}), 7.87$ (d, $7.9 \mathrm{~Hz}, 4 \mathrm{H}), 7.76$ (d, $7.9 \mathrm{~Hz}, 4 \mathrm{H})$.

${ }^{13} \mathrm{C}$ NMR (150 MHz, CDCl 3 , $)$ : 145.7, 135.6, 132.2, 131.6, 131.3, 128.0, 128.0, 127.5, 125.4, 125.2, 125.0, 118.8, 111.3.

HRMS (DART, positive, $\mathrm{m} / \mathrm{z}$ ): calcd. for $\mathrm{C}_{30} \mathrm{H}_{16} \mathrm{~N}_{2}{ }^{+\bullet}[\mathrm{M}]^{+\bullet}: 404.1313$; found: 404.1321 .

1,8-bis(4-(tert-butyl)phenyl)benzo[e]pyrene (compound $\mathbf{4 g}$ ). Following general procedure $D$, the<smiles></smiles>
title compound was synthesized from $0.05 \mathrm{~g}(0.0457 \mathrm{mmol}) \mathrm{of} \mathbf{2 g}$. The product was isolated by preparative thin layer chromatography using $5 \% \mathrm{Et}_{2} \mathrm{O}$ in hexanes as eluent to give $0.021 \mathrm{~g}$ of the title product as a yellow solid (89\%). ${ }^{1} \mathrm{H}$ NMR $\left(400 \mathrm{MHz} \mathrm{CDCl}_{3}, \delta\right): 8.13(\mathrm{~d}, J=7.9 \mathrm{~Hz}, 2 \mathrm{H}), 8.00(\mathrm{~s}, 2 \mathrm{H}), 7.94$ (d, 7.9 $\mathrm{Hz}, 2 \mathrm{H}), 7.82-7.80(\mathrm{~m}, 2 \mathrm{H}), 7.58(\mathrm{~d}, J=8.3 \mathrm{~Hz}, 4 \mathrm{H}), 7.51(\mathrm{~d}, J=8.3 \mathrm{~Hz}, 4 \mathrm{H})$, 6.94-6.92 (m, 2H), $1.41(\mathrm{~s}, 18 \mathrm{H})$.

${ }^{13} \mathrm{C}$ NMR (150 MHz, $\left.\mathrm{CDCl}_{3}, \delta\right): 150.0,141.8,137.0,131.8,130.4,130.3,130.0$, $129.4,128.1,126.7,126.2,126.0,125.8,124.8,34.6,31.4$. 
HRMS (DART, positive, m/z): calcd. for $\mathrm{C}_{40} \mathrm{H}_{36}{ }^{+\bullet}[\mathrm{M}]^{+\bullet}:$ 516.2817; found: 516.2823 .

1,7-bis(4-(tert-butyl)phenyl)perylene (compound 4h). Following a modified version of general

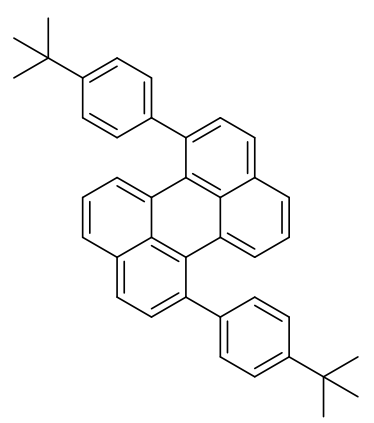
procedure $\mathrm{D}$ (using $2 \mathrm{M} \mathrm{HCl}$ for 5 hours in place of TFA with which small amounts of side products formed), the title compound was synthesized from $0.05 \mathrm{~g}(0.0457 \mathrm{mmol})$ of $\mathbf{2} \mathbf{h}$. The product was isolated by column chromatography using $1 \% \mathrm{Et}_{2} \mathrm{O}$ in hexanes as eluent to give to give $0.02 \mathrm{~g}$ of the title product as a bright yellow solid (85\%).

${ }^{1} \mathrm{H}$ NMR (400 MHz, $\left.\mathrm{CDCl}_{3}, \delta\right): 7.69(\mathrm{~d}, J=8.5 \mathrm{~Hz}, 2 \mathrm{H}), 7.54(\mathrm{~d}, J=7.8 \mathrm{~Hz}$ $2 \mathrm{H}), 7.46-7.37(\mathrm{~m}, 12 \mathrm{H}), 7.00(\mathrm{t}, J=7.8 \mathrm{~Hz}, 2 \mathrm{H}), 1.39(\mathrm{~s}, 18 \mathrm{H})$.

${ }^{13} \mathrm{C}$ NMR (150 MHz, $\left.\mathrm{CDCl}_{3}, \delta\right): 150.1,141.5,137.8,132.8,131.6,130.9$, 130.8, 129.1, 129.0, 128.9, 126.9, 126.4, 126.2, 125.1, 34.6, 31.4.

HRMS (DART, positive, $\mathrm{m} / \mathrm{z}$ ): calcd. for calcd. for $\mathrm{C}_{40} \mathrm{H}_{36}{ }^{+\bullet}[\mathrm{M}]^{+\bullet}: 516.2817$; found: 516.2832.

1,10-diphenyldibenzo[ $c d, I m]$ perylene (compound 4i). Following general procedure $D$, the title

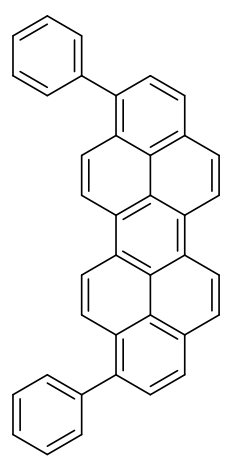
compound was synthesized from crude reaction mixture $\mathbf{2} \mathbf{i}$. The product was isolated by preparative thin layer chromatography using 5\% EtOAc in hexanes as eluent to give $0.033 \mathrm{~g}$ of the title product as a dark yellow solid (>95\% from 0.0710 mmol $2 \mathbf{i}$ calculated in crude).

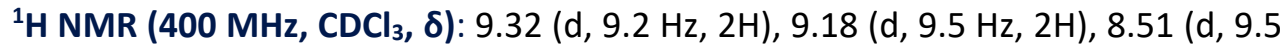
$\mathrm{Hz}, 2 \mathrm{H}), 8.45(\mathrm{~d}, 9.2 \mathrm{~Hz}, 2 \mathrm{H}), 8.42(\mathrm{~d}, 7.8 \mathrm{~Hz}, 2 \mathrm{H}), 8.13(\mathrm{~d}, 7.8 \mathrm{~Hz}, 2 \mathrm{H}), 7.74-7.72(\mathrm{~m}$, $4 \mathrm{H}), 7.64-7.60(\mathrm{~m}, 4 \mathrm{H}), 7.55-7.52(\mathrm{~m}, 2 \mathrm{H})$.

${ }^{13} \mathrm{C}$ NMR (150 MHz, $\left.\mathrm{CDCl}_{3}, \delta\right): 141.3,138.0,130.7,130.7,128.7,128.4,127.9$, $127.8,127.3,125.8,125.7,125.5,125.1,124.9,123.3,122.9$ (one sp ${ }^{2}$ signals not observed due to overlapping).

HRMS (DART, positive, $\mathrm{m} / \mathrm{z}$ ): calcd. for $\mathrm{C}_{38} \mathrm{H}_{22}{ }^{+\bullet}[\mathrm{M}]^{+\bullet}: 478.1721$; found: 478.1726 . 


\section{Synthesis of extended polyarene 10}

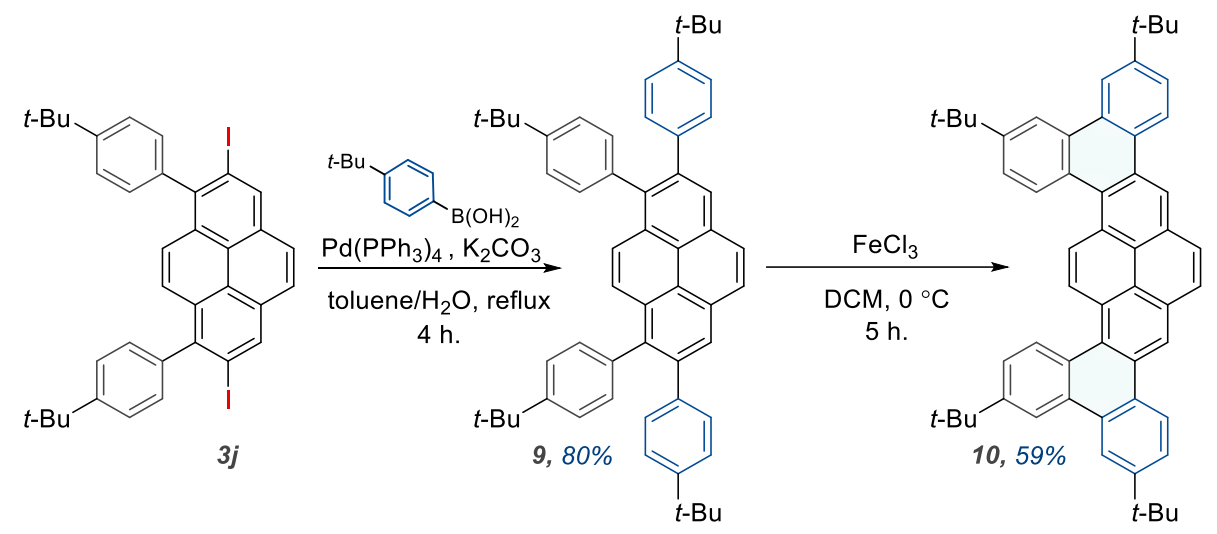

1,2,7,8-tetrakis(4-(tert-butyl)phenyl)pyrene (compound 9). A mixture of 3j (0.45 g; $0.6263 \mathrm{mmol})$,<smiles></smiles>
4-tert-butylphenylboronic acid (0.3345 g; $1.88 \mathrm{mmol}), \mathrm{K}_{2} \mathrm{CO}_{3}(0.1731 \mathrm{~g} ; 1.25$ $\mathrm{mmol})$, and $\mathrm{Pd}\left(\mathrm{PPh}_{3}\right)_{4}(0.087 \mathrm{~g} ; 0.125 \mathrm{mmol})$ in a dry, outgassed toluene/ $\mathrm{H}_{2} \mathrm{O}$ mixture $(6.7 \mathrm{~mL}$ and $1.0 \mathrm{~mL}$ respectively; $0.05 \mathrm{M})$ was heated to reflux under vigorous stirring for 4 hours under argon atmosphere. After this time, the reaction mixture was passed through a bed of $\mathrm{Na}_{2} \mathrm{SO}_{4}$ atop celite and concentrated under vacuum. The product was isolated from the crude reaction mixture by column chromatography using $1.5 \%$ AcOEt in hexanes as eluent to give $0.41 \mathrm{~g}$ of the title product as a white solid (80\%).

${ }^{1} \mathrm{H} \mathrm{NMR}\left(400 \mathrm{MHz}, \mathrm{CDCl}_{3}, \delta\right): 8.27(\mathrm{~s}, 2 \mathrm{H}), 8.11(\mathrm{~s}, 2 \mathrm{H}), 7.91(\mathrm{~s}, 2 \mathrm{H}), 7.30-7.16$ $(\mathrm{m}, 16 \mathrm{H}), 1.32(\mathrm{~s}, 18 \mathrm{H}), 1.30(\mathrm{~s}, 18 \mathrm{H})$.

${ }^{13} \mathrm{C} \mathrm{NMR}\left(150 \mathrm{MHz}, \mathrm{CDCl}_{3}, \delta\right):$ 149.4, 148.9, 139.4, 139.3, 136.3, 131.4, 130.6, 130.1, 129.4, 127.5, 126.6, 126.2, 124.4, 124.3, 124.0, 34.4, 34.3, 31.4, 31.3. HRMS (DART, positive, $\mathrm{m} / \mathrm{z}$ ): calcd. for $\mathrm{C}_{56} \mathrm{H}_{59}{ }^{+}[\mathrm{M}+\mathrm{H}]^{+}:$731.4616; found: 731.4595 .

2,11,14,21-tetra-tert-butylpentabenzo[a,c,m,o,rst]pentaphene (compound 10). An argon filled

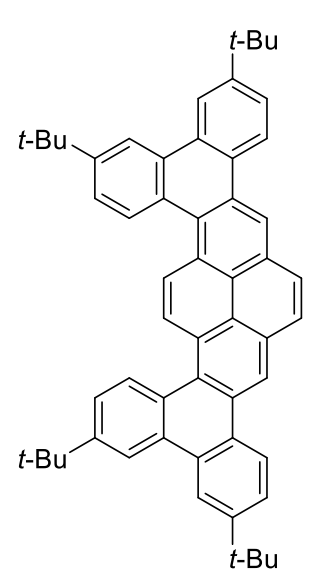
Schlenk tube equipped with a stir bar and loaded with $\mathrm{FeCl}_{3}(0.4 \mathrm{~g}, 2.46 \mathrm{mmol})$ was cooled to $-5^{\circ} \mathrm{C}$. A suspension of 9 ( $\left.0.15 \mathrm{~g} ; 0.2052 \mathrm{mmol}\right)$ in dry methylene chloride was added in three portions $(20 \mathrm{~mL}$ methylene chloride total) and the reaction mixture was left stirring at $-5^{\circ} \mathrm{C}$ for 7 hours. The reaction was quenched with $\mathrm{MeOH}(18 \mathrm{~mL})$ and the reaction mixture transferred to a separatory funnel where it was washed with $\mathrm{H}_{2} \mathrm{O}(90 \mathrm{~mL})$. Then, the aqueous layer was separated and extracted with methylene chloride $(20 \mathrm{~mL} \times 3)$. The combined organic layer was dried with $\mathrm{MgSO}_{4}$ and the solvent was removed under reduced pressure. The product was isolated from the crude reaction mixture by column chromatography using $2 \% \mathrm{Et}_{2} \mathrm{O}$ in hexanes as eluent to give $0.88 \mathrm{~g}$ of the title product as a bright-yellow solid (59\%).

${ }^{1} \mathrm{H}$ NMR $\left(400 \mathrm{MHz}, \mathrm{CDCl}_{3}, \delta\right): 9.28(\mathrm{~s}, 2 \mathrm{H}), 9.22(\mathrm{~s}, 2 \mathrm{H}), 9.18(\mathrm{~d}, 8.5 \mathrm{~Hz}, 2 \mathrm{H}), 8.88(\mathrm{~d}, 8.5 \mathrm{~Hz}, 2 \mathrm{H}), 8.79$ $(\mathrm{d}, 11.0 \mathrm{~Hz}, 2 \mathrm{H}), 8.78(\mathrm{~d}, 11.0 \mathrm{~Hz}, 2 \mathrm{H}), 8.23(\mathrm{~s}, 2 \mathrm{H}), 7.90-7.84(\mathrm{~m}, 4 \mathrm{H}), 1.62(\mathrm{~s}, 18 \mathrm{H}), 1.58(\mathrm{~s}, 18 \mathrm{H})$. 
$\left.{ }^{13} \mathrm{C} \mathrm{NMR} \mathrm{(150} \mathrm{MHz,} \mathrm{CDCl} 3,8\right): 150.2,149.7,130.8,130.2,130.2,130.0,128.2,127.9,127.9,127.8$, 127.1, 126.7, 125.5, 125.3, 124.9, 124.1, 123.8, 119.3, 119.1, 119.0, 35.1, 35.1, 31.5, 31.5. MS (MALDI-TOF, positive, $\mathrm{m} / \mathrm{z}$ ): calcd. for $\mathrm{C}_{56} \mathrm{H}_{54}{ }^{+}[\mathrm{M}]^{+}$: 726.4225; found: 726.428 .

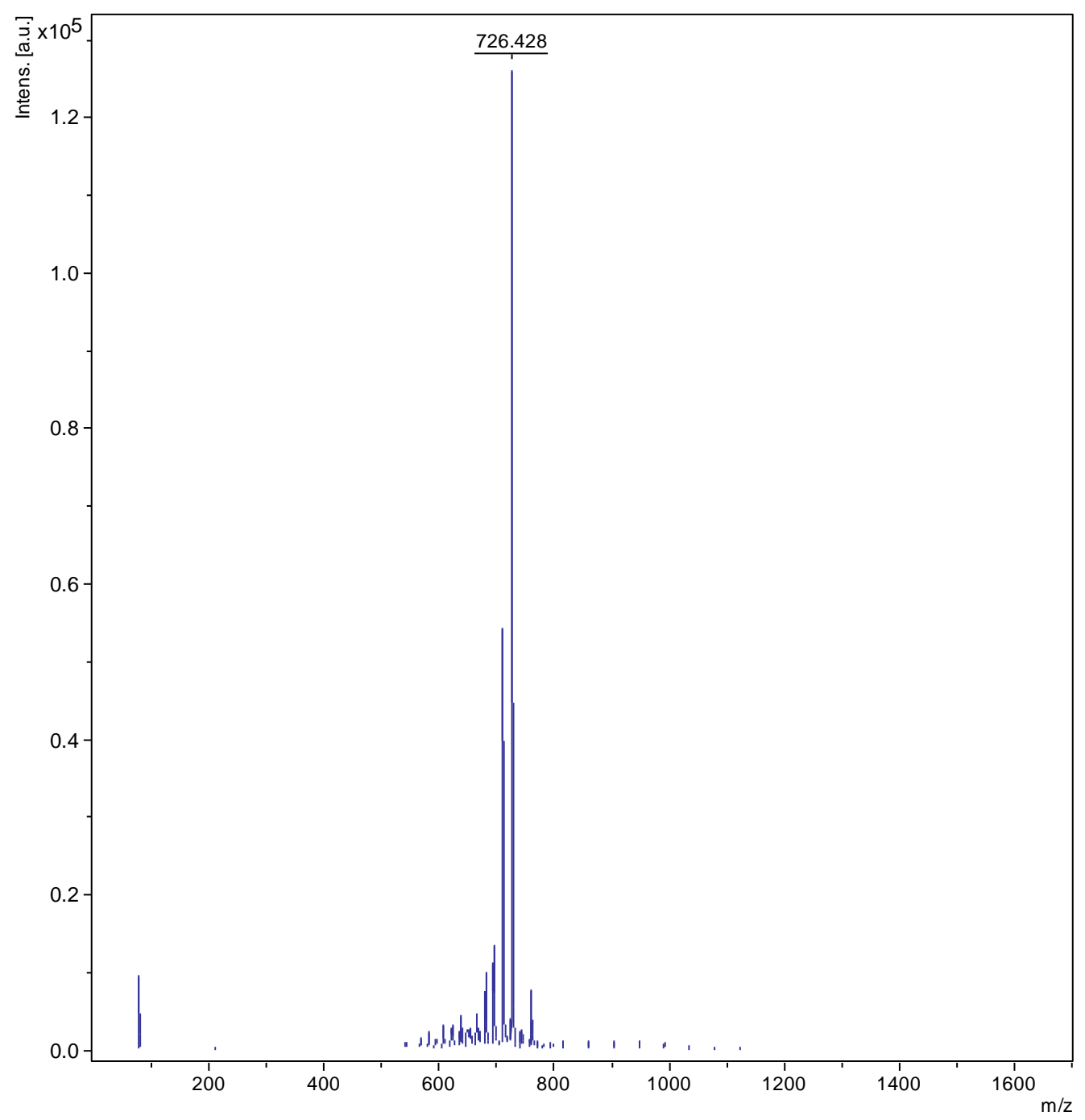

Figure S12. MALDI mass spectrum of $\mathbf{1 0}$. Minor ions at lower $\mathrm{m} / \mathrm{z}$ are due to fragmentation of the alkyl groups. 
Optimization conditions for the Sn-promoted double peri-annulation

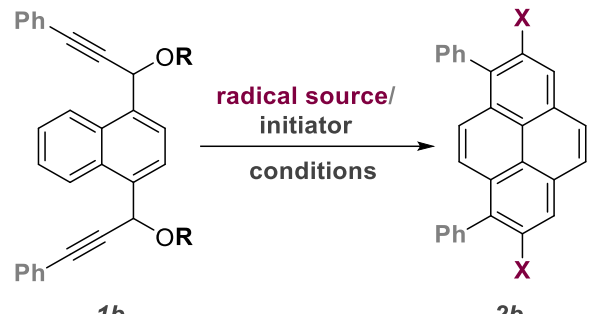

$1 b$

$2 b$

\begin{tabular}{|c|c|c|c|}
\hline Entry & Reagent/Initiator & Conditions & Yield ( $\%$ conversion) \\
\hline $1^{[a]}$ & $\mathrm{Bu}_{3} \mathrm{SnH}(2.0) / \mathrm{AIBN}(1.0)$ & toluene, reflux, $15 \mathrm{~h}$. & $17 \%(100)$ \\
\hline $2^{[a]}$ & $\mathrm{Bu}_{3} \mathrm{SnH}(2.2) / \mathrm{AIBN}$ (0.2) & toluene, reflux, $15 \mathrm{~h}$. & $0 \%(79)$ \\
\hline $3^{[\mathrm{a}]}$ & $\mathrm{Bu}_{3} \mathrm{SnH}(2.2) / \mathrm{AIBN}(\mathbf{0 . 4 )}$ & toluene, reflux, $15 \mathrm{~h}$. & $7 \%(89)$ \\
\hline $4^{[a]}$ & $\mathrm{Bu}_{3} \mathrm{SnH}(2.2) / \mathrm{AIBN}(\mathbf{0 . 6})$ & toluene, reflux, $15 \mathrm{~h}$. & $11 \%(93)$ \\
\hline $5^{[a]}$ & $\mathrm{Bu}_{3} \mathrm{SnH}(2.2) / \mathrm{AIBN}(\mathbf{0 . 8 )}$ & toluene, reflux, $15 \mathrm{~h}$. & $21 \%(100)$ \\
\hline $6^{[a]}$ & $\mathrm{Bu}_{3} \mathrm{SnH}(2.2) / \mathrm{AIBN}(\mathbf{1 . 6})$ & toluene, reflux, $15 \mathrm{~h}$. & $13 \%(100)$ \\
\hline $7^{[a]}$ & $\mathrm{Bu}_{3} \mathrm{SnH}(2.2) / \mathrm{AIBN}(0.8)$ & toluene, $90^{\circ} \mathbf{C}, 15 \mathrm{~h}$. & $14 \%(81)$ \\
\hline $8^{[a]}$ & $\mathrm{Bu}_{3} \mathrm{SnH}(2.2) / \mathrm{AIBN}(0.8)$ & toluene, $105^{\circ} \mathbf{C}, 4.5 \mathrm{~h}$ & $10 \%(87)$ \\
\hline $9^{[a]}$ & $\mathrm{Bu}_{3} \mathrm{SnH}(4.0) / \mathrm{AIBN}(0.8)$ & toluene, reflux, $15 \mathrm{~h}$. & $15 \%(100)$ \\
\hline $10^{[a]}$ & $\mathrm{Bu}_{3} \mathrm{SnH}(2.2) / \mathrm{AIBN}(0.8)$ & MeCN, reflux, $1 \mathrm{~h}$. & $0 \%(100)$ \\
\hline $11^{[a]}$ & $\mathrm{Bu}_{3} \mathrm{SnH}(2.2) / \mathbf{A C H N}(\mathbf{0 . 4 )}$ & toluene, reflux, $15 \mathrm{~h}$. & $13 \%(100)$ \\
\hline $12^{[a]}$ & $\mathrm{Bu}_{3} \mathrm{SnH}(2.2) / \mathrm{ACHN}(\mathbf{0 . 8 )}$ & toluene, reflux, $15 \mathrm{~h}$. & $22 \%(100)$ \\
\hline $13^{[a]}$ & $\mathrm{Bu}_{3} \mathrm{SnH}(2.2) / \mathrm{ACHN}$ (1.2) & toluene, reflux, $15 \mathrm{~h}$. & $26 \%(100)$ \\
\hline $14^{[a]}$ & $\mathrm{Bu}_{3} \mathrm{SnH}(2.2) / \mathrm{ACHN}(\mathbf{1 . 6})$ & toluene, reflux, $15 \mathrm{~h}$. & $31 \%(100)$ \\
\hline $15^{[a]}$ & $\mathrm{Bu}_{3} \mathrm{SnH}$ (2.2)/ACHN (3.2) & toluene, reflux, $15 \mathrm{~h}$. & $30 \%(100)$ \\
\hline $16^{[a]}$ & $\mathrm{Bu}_{3} \mathrm{SnH}$ (2.2)/ACHN (6.4) & toluene, reflux, $15 \mathrm{~h}$. & $31 \%(100)$ \\
\hline $17^{[\mathrm{b}]}$ & $\mathrm{Bu}_{3} \mathrm{SnH}(2.2) / \mathrm{ACHN}(1.6)$ & toluene, reflux, $15 \mathrm{~h}$. & $36 \%(100)$ \\
\hline $18^{[\mathrm{c}]}$ & $\mathrm{Bu}_{3} \mathrm{SnH}(2.2) / \mathrm{ACHN}(1.6)$ & toluene, reflux, $15 \mathrm{~h}$. & $18 \%(100)$ \\
\hline $19^{[b]}$ & $\mathrm{Bu} \mathrm{SnH}_{\mathrm{S}}(2.2) / \mathrm{ACHN}(1.6)$ & toluene, reflux, $\mathbf{5} \mathbf{~ h}$. & $38 \%(100)$ \\
\hline $20^{[b]}$ & $\mathrm{Bu}_{3} \mathrm{SnH}(4.0) / \mathrm{ACHN}(1.6)$ & toluene, reflux, $15 \mathrm{~h}$. & $37 \%(100)$ \\
\hline $21^{[\mathrm{b}]}$ & $\mathbf{P h}_{3} \mathrm{SnH}(2.2) / \mathrm{ACHN}(1.6)$ & toluene, reflux, $5 \mathrm{~h}$. & $33 \%(100)$ \\
\hline $22^{[b]}$ & $\mathrm{Bu}_{3} \mathrm{SnH}(2.2) /$ benzoylperoxide (1.6) & toluene, reflux, $15 \mathrm{~h}$. & trace $(65)$ \\
\hline $23^{[b]}$ & $\mathrm{Bu}_{3} \mathrm{SnH}(2.2) /$ benzoylperoxide (1.6) & benzene, reflux, $15 \mathrm{~h}$. & trace $(46)$ \\
\hline $24^{[b]}$ & $\mathrm{TMS}_{3} \mathrm{SiH}(2.2) / \mathrm{ACHN}(0.8)$ & toluene, reflux, $24 \mathrm{~h}$. & $0 \%(64)$ \\
\hline $25^{[b]}$ & $\mathrm{TMS}_{3} \mathrm{SiH}(2.2) / \mathrm{AIBN}(\mathbf{0 . 8 )}$ & toluene, reflux, $24 \mathrm{~h}$. & $0 \%(58)$ \\
\hline $26^{[b]}$ & Togni II (3.0)/Bu ${ }_{4} \mathrm{NI}(0.2)$ & 1,4-dioxane, $80^{\circ} \mathrm{C}, 48 \mathrm{~h}$ & $0 \%(53)$ \\
\hline $27^{[\mathrm{b}]}$ & Isopropylboronic acid (3.5)/Mn(acac) 3 (0.8) & toluene, $105^{\circ} \mathrm{C}, 15 \mathrm{~h}$. & $0 \%(0)$ \\
\hline $28^{[b],[d]}$ & $\mathrm{Bu}_{3} \mathrm{SnH}(2.2) / \mathrm{ACHN}(1.6)$ & toluene, reflux, $22 \mathrm{~h}$. & $18 \%(100)$ \\
\hline $29^{[b],[e]}$ & $\mathrm{Bu}_{3} \mathrm{SnH}(2.2) / \mathrm{ACHN}(1.6)$ & toluene, reflux, $22 \mathrm{~h}$. & $7 \%(100)$ \\
\hline $30^{[b],[f]}$ & $\mathrm{Bu}_{3} \mathrm{SnH}(2.2) / \mathrm{ACHN}(1.6)$ & toluene, reflux, 5 h. & $21 \%(100)$ \\
\hline $31^{[\mathrm{b}]}$ & $\mathrm{Bu}_{3} \mathrm{SnH}(2.2) / \mathrm{ACHN}(1.6)$ & $\mathrm{PhCF}_{3}$, reflux, $15 \mathrm{~h}$. & $29 \%(100)$ \\
\hline $32^{[b]}$ & $\mathrm{Bu}_{3} \mathrm{SnH}(2.2) / \mathrm{ACHN}(1.6)$ & ODCB, reflux, $15 \mathrm{~h}$. & $10 \%(100)$ \\
\hline $33^{[b]}$ & $\mathrm{Bu}_{3} \mathrm{SnH}(2.2) / \mathrm{ACHN} / \mathrm{AIBN}(\mathbf{0 . 8 / 0 . 8 )}$ & toluene, reflux, $15 \mathrm{~h}$. & $28 \%(100)$ \\
\hline $34^{[b],[g]}$ & $\mathrm{Bu}_{3} \mathrm{SnH}(2.2) / \mathrm{ACHN}(1.6)$ & toluene, reflux, $17 \mathrm{~h}$. & $\mathbf{N} / \mathbf{A}(0)$ \\
\hline
\end{tabular}

Table S1. Optimization table for the double peri-annulation on the model system. Initial Starting material concentration: [a] $0.04 \mathrm{M}$, [b] $0.02 \mathrm{M}$, and [c] $0.01 \mathrm{M}$. [d] $\mathrm{R}=\mathrm{H}$, [e] $\mathrm{R}=\mathrm{C}(\mathrm{O}) \mathrm{CH}_{3}$, [f] $\mathrm{R}=i \mathrm{Pr}$. [g] stability test: no decomposition of distannylarene observed. Radical source/initiator mixtures added over the course of 3 hours via syringe pump. 


\section{Photophysical Characterization}

\section{Absorption spectroscopy}

The UV-visible spectra were recorded using an Agilent 8453 UV-Vis photo diode array spectrophotometer with a special optical glass $1 \mathrm{~cm} \times 1 \mathrm{~cm}$ cuvette.

Steady state emission:

An Edinburgh FLS980 fluorescence spectrometer was used to collect emission data at room temperature. A housed $450 \mathrm{~W}$ Xe lamp/single grating $(1800 \mathrm{l} / \mathrm{mm}, 250 \mathrm{~nm}$ blaze) Czerny-Turner monochromator was used to excite the samples. Emission from the sample were passed through a single grating ( $1800 \mathrm{l} / \mathrm{mm}, 500 \mathrm{~nm}$ blaze) Czerny-Turner monochromator ( $0.75 \mathrm{~nm}$ bandwidth) and then detected by a Peltier-cooled Hamamatsu R928 photomultiplier tube. Samples were excited at $340 \mathrm{~nm}$. Room temperature emission spectra were recorded with solutions in a $1 \times 1 \mathrm{~cm}$ cuvette.

Time-Resolved Emission:

Data were collected at room temperature using an Edinburgh FLS980 spectrometer. The dynamics of emission decay were monitored by using the FLS980's time-correlated single-photon counting capability (1024 channels; 100 ns window) with data collection for 10,000 counts. Excitation was provided by an Edinburgh EPL-360 picosecond pulsed light emitting diode (360 $10 \mathrm{~nm}$, pulse width - $892 \mathrm{ps)}$ operated at $10 \mathrm{MHz}$. Emission from the sample was passed through a single grating (1800 $\mathrm{l} / \mathrm{mm}, 500 \mathrm{~nm}$ blaze) Czerny-Turner monochromator ( $0.75 \mathrm{~nm}$ bandwidth) and finally detected by a peltier-cooled Hamamatsu R928 photomultiplier tube. Kinetics were fit with a single exponential function by using Edinburgh software package. Samples were the same as described for steady-state emission measurements.

Absolute Emission quantum yields:

Absolute Emission quantum yields were acquired using a Quantaurus Absolute PL Quantum Yield Spectrometer (Hamamatsu). Samples were prepared in $\mathrm{CH}_{2} \mathrm{Cl}_{2}$ with an $\mathrm{A}<0.1$ in a $1 \mathrm{~cm}$ cuvette) and then transfer to a $12.5 \mathrm{~cm}$ long, $0.8 \mathrm{~cm}$ diameter quartz tube with a round bottom and a rubber septa cap for data acquisition. A similar tube with the same volume of $\mathrm{CH}_{2} \mathrm{Cl}_{2}$ only was used as a reference. Samples and reference were excited with $360 \mathrm{~nm}$ light and the excitation and emission intensities recorded at $1 \mathrm{~nm}$ step intervals from 340 to $900 \mathrm{~nm}$. Quantum yields were then calculated using the De Mello method. ${ }^{3}$ 


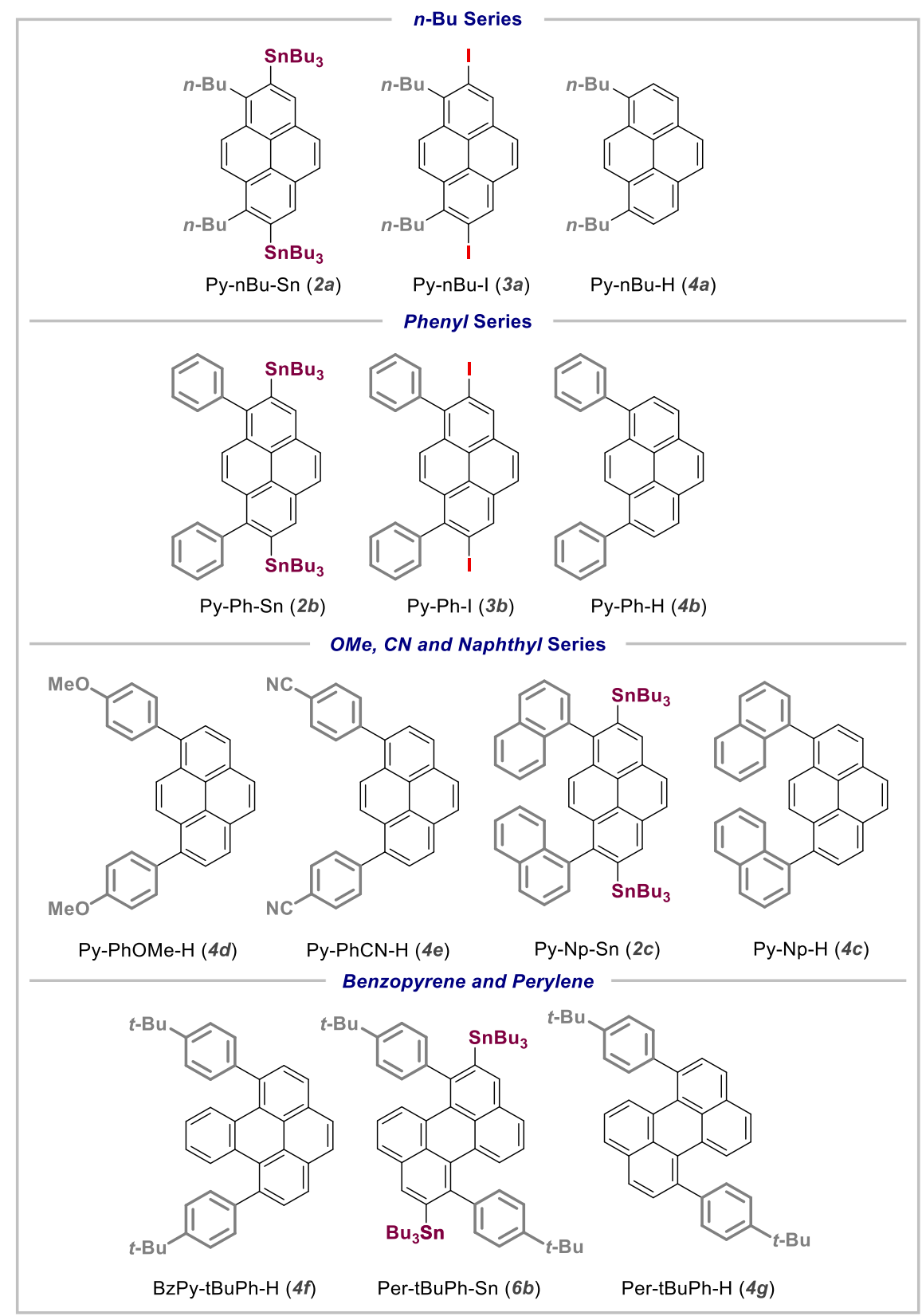

Figure S13. Compounds and their corresponding codes (for clarity) for photophysics section. 
Room temperature absorption and emission overlay Pyrene $\left(\lambda_{\mathrm{ex}}=340 \mathrm{~nm}\right)$

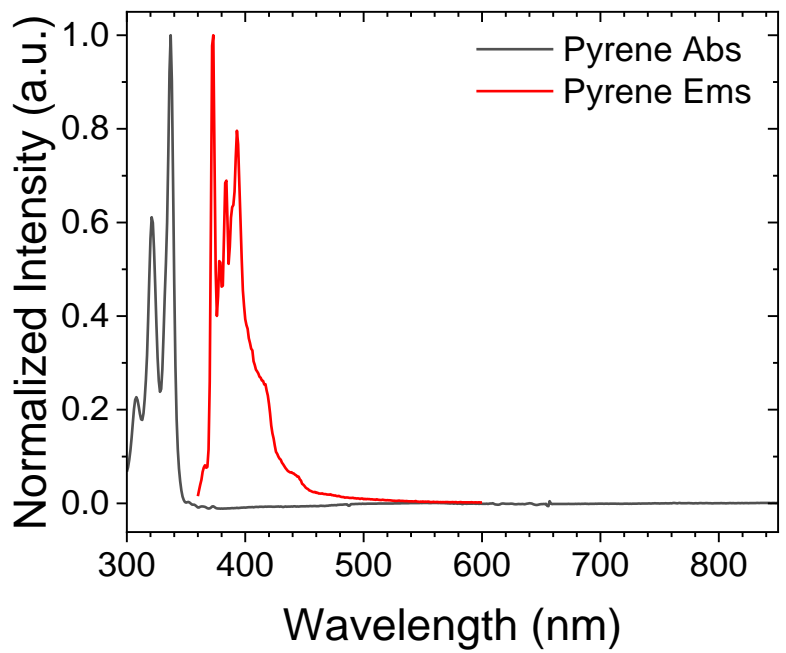

Absorption (all spectra in $\mathrm{CH}_{2} \mathrm{Cl}_{2}$ )
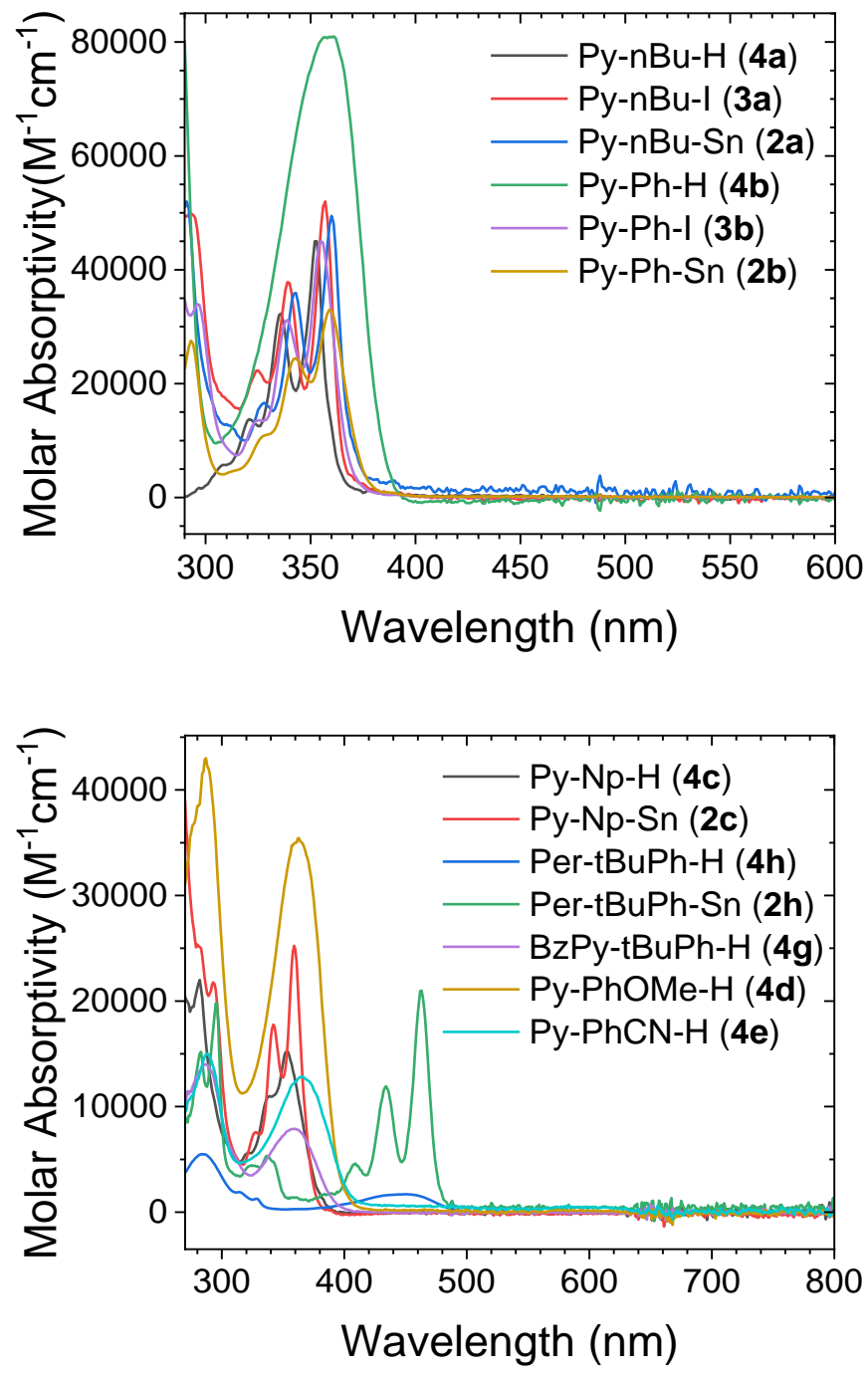
Room temperature absorption and emission $\left(\lambda_{\mathrm{ex}}=340 \mathrm{~nm}\right) n$-Bu series
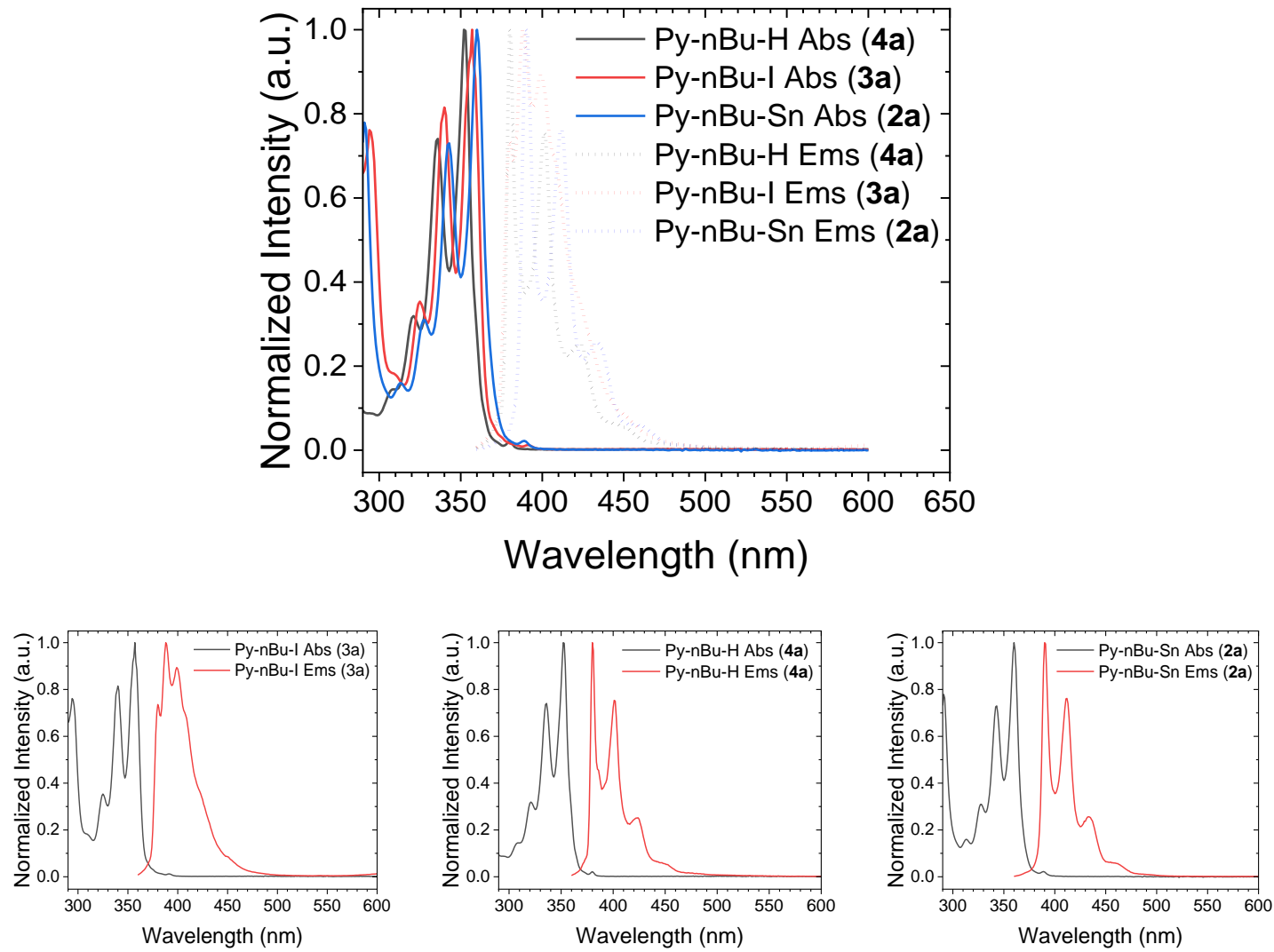

Room temperature absorption $\mathrm{Ph}$ series

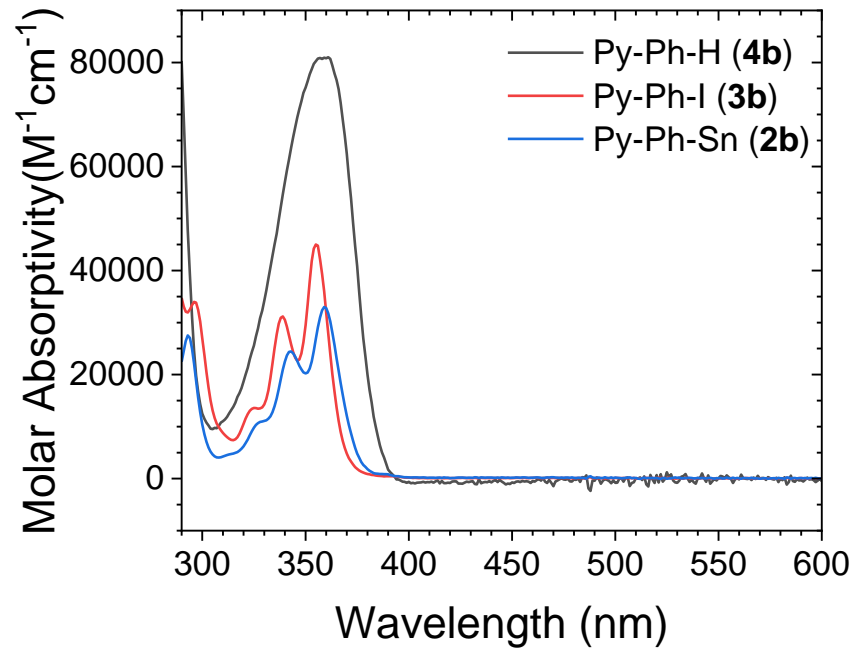


Room temperature absorption and emission $\left(\lambda_{\mathrm{ex}}=360 \mathrm{~nm}\right)$ for naphthyl series
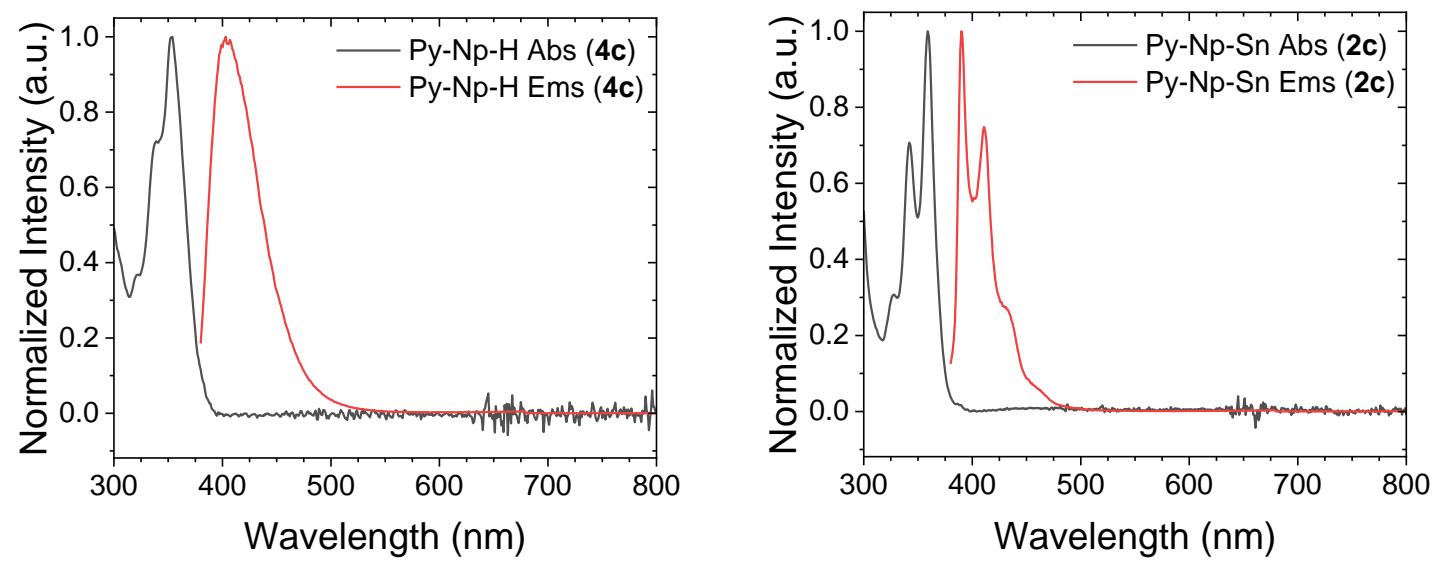

Room temperature absorption and emission $\left(\lambda_{\mathrm{ex}}=360 \mathrm{~nm}\right)$ for $\mathrm{CN}$ and OMe phenyl pyrenes.
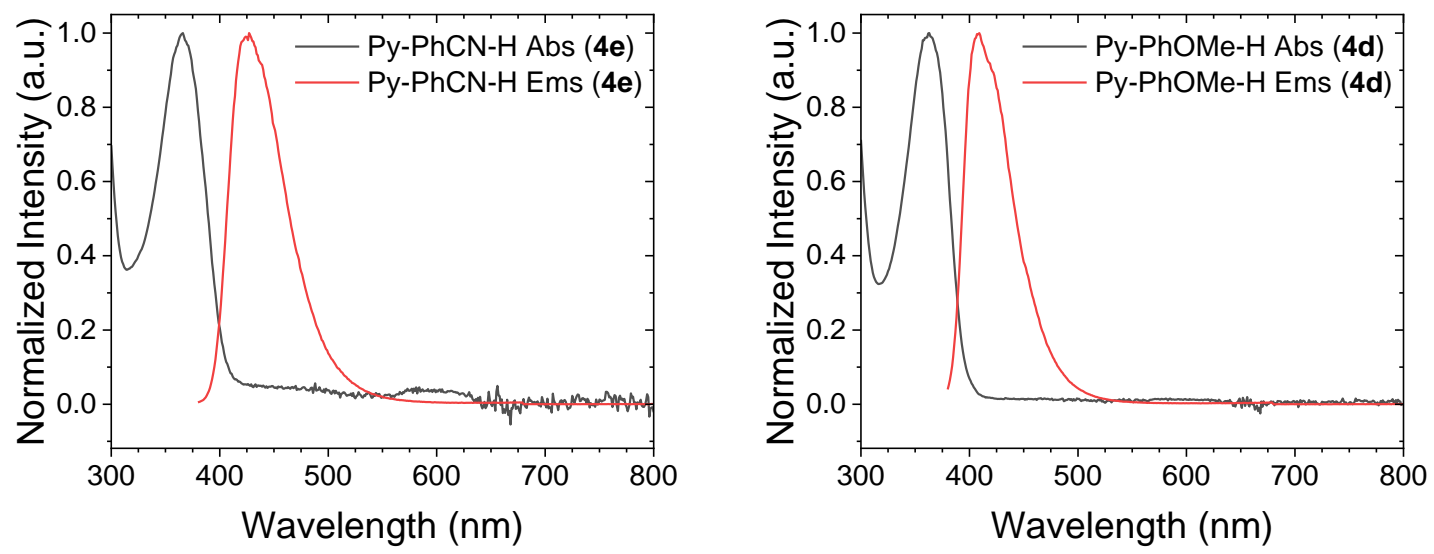
Room temperature absorption and emission $\left(\lambda_{\mathrm{ex}}=360 \mathrm{~nm}\right)$ for substituted benzopyrene

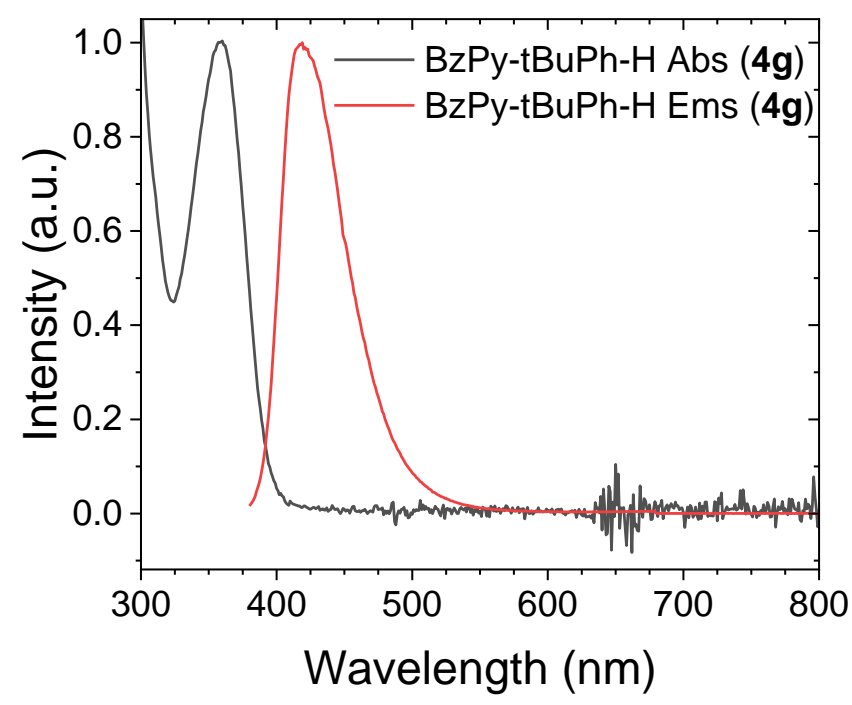

Room temperature absorption and emission $\left(\lambda_{\mathrm{ex}}=450 \mathrm{~nm}\right)$ for perylene series
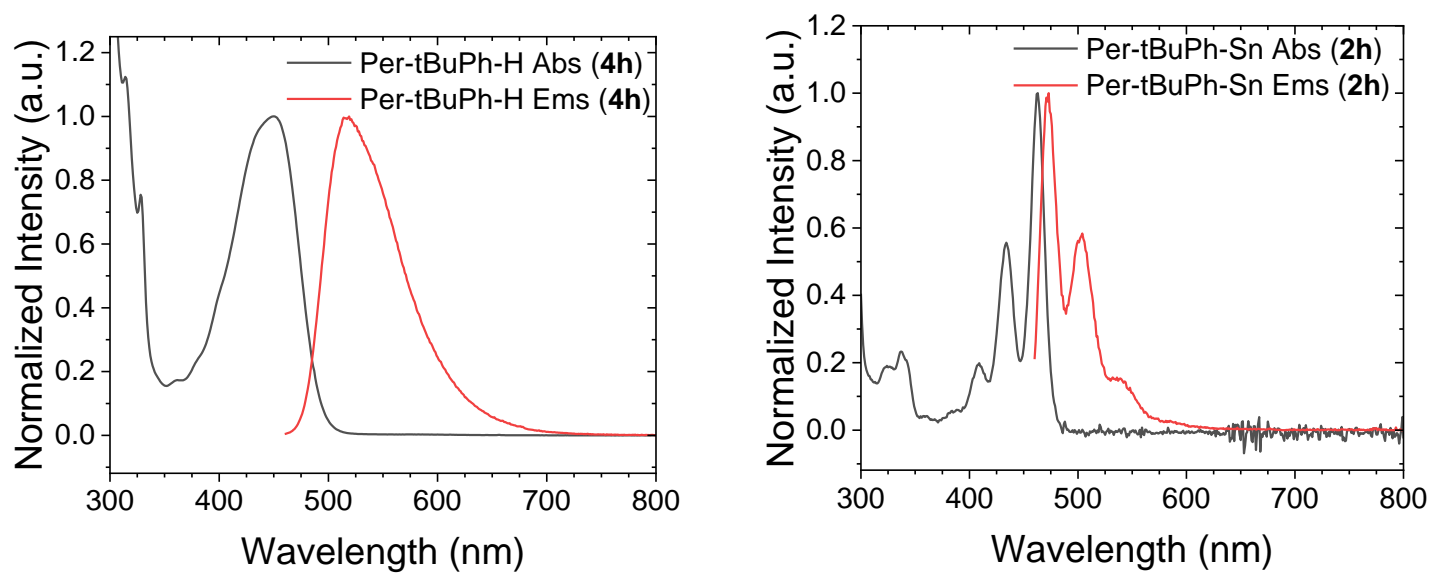
Room temperature time-resolved emission $\left(\lambda_{\mathrm{ex}}=360 \mathrm{~nm}\right)$ for all compounds

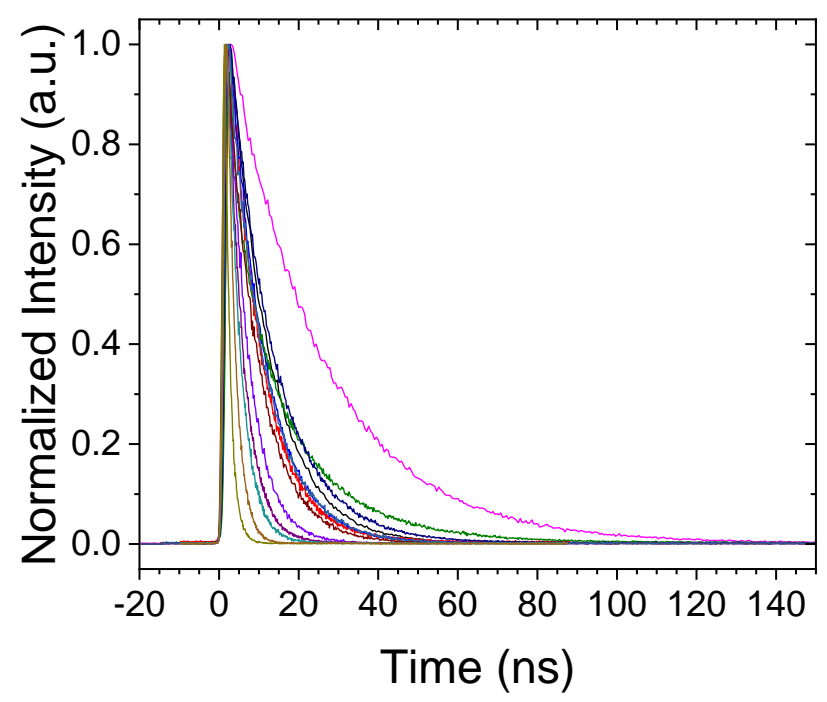

$\mathrm{Py}-\mathrm{Ph}-\mathrm{H}(\mathbf{4 b})$

Py-Ph-I (3b)

Py-Ph-Sn (2b)

Py-nBu-H (4a)

Py-nBu-I (3a)

Py-nBu-Sn (2a)

$\mathrm{Py}-\mathrm{Np}-\mathrm{H}(\mathbf{4 c})$

Py-Np-Sn (2c)

Per-tBuPh-H (4h)

Per-tBuPh-Sn (2h)

BzPy-tBuPh-H (4g)

Py-PhOMe-H (4d)

Py-PhCN-H (4e)

Room temperature time-resolved emission $\left(\lambda_{\mathrm{ex}}=360 \mathrm{~nm}\right) n$-Bu series

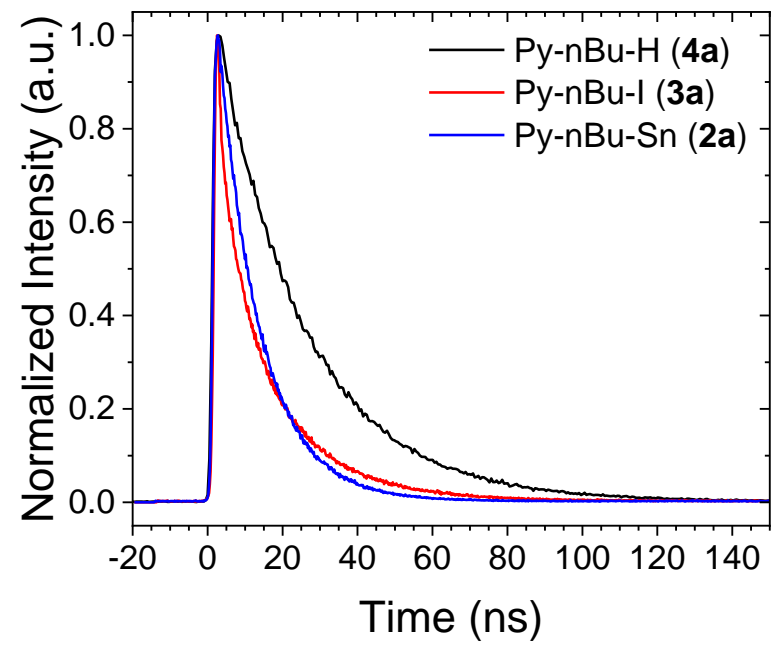


Room temperature time-resolved emission $\left(\lambda_{\mathrm{ex}}=360 \mathrm{~nm}\right)$ phenyl series

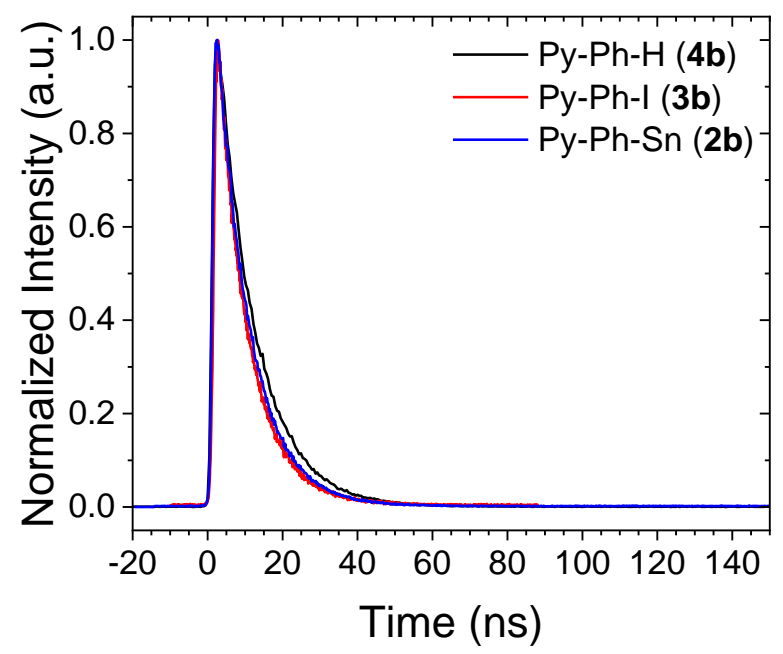

Room temperature time-resolved emission $\left(\lambda_{\mathrm{ex}}=360 \mathrm{~nm}\right)$ naphthyl series

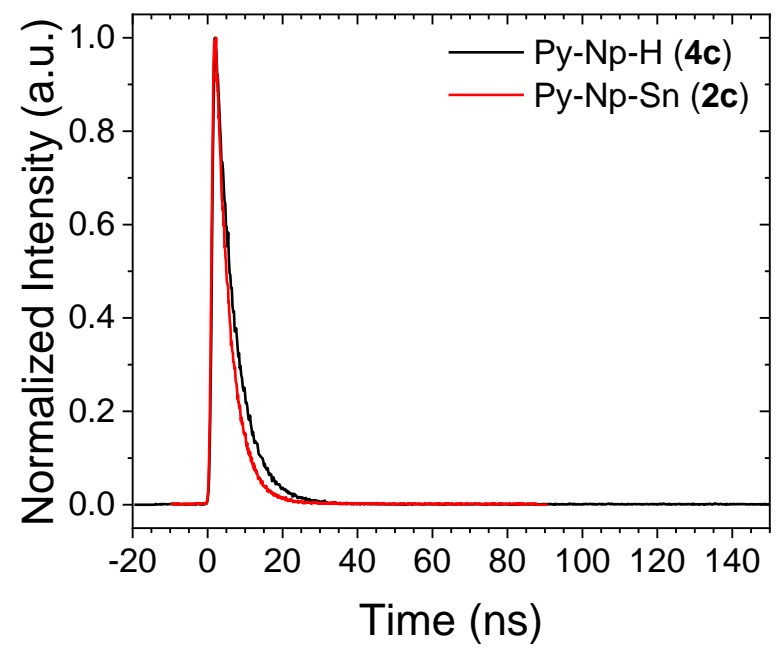


Room temperature time-resolved emission $\left(\lambda_{\mathrm{ex}}=360 \mathrm{~nm}\right)$ perylene series

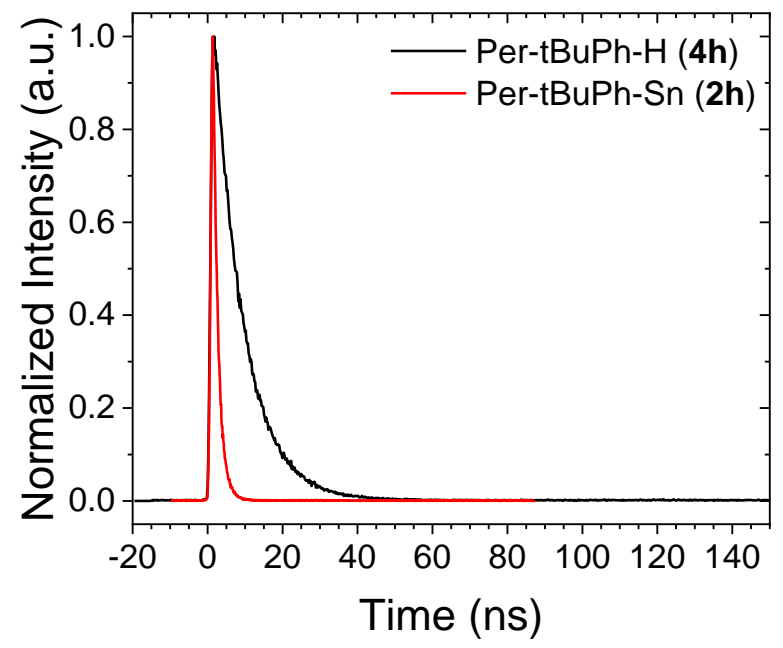

Room temperature time-resolved emission $\left(\lambda_{\mathrm{ex}}=360 \mathrm{~nm}\right)$ substituted phenylpyrene series

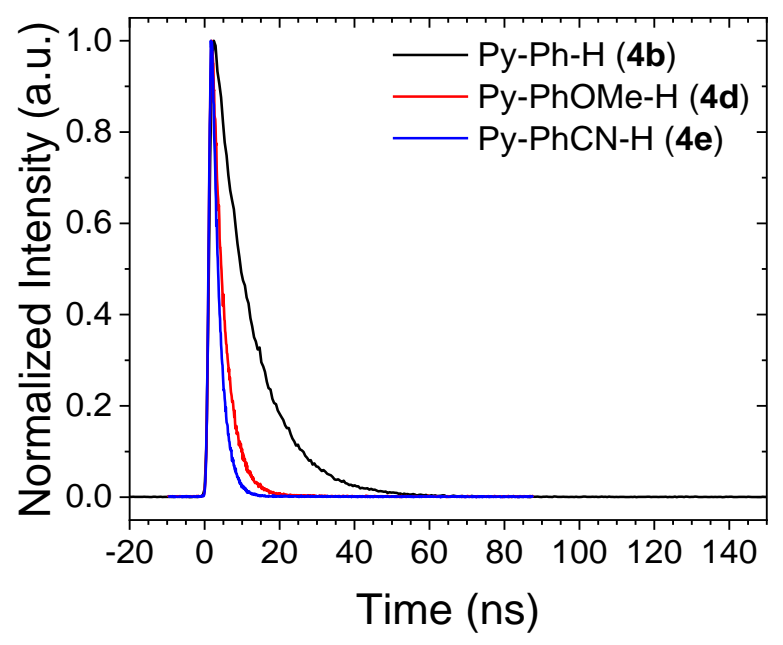


Room temperature time-resolved emission $\left(\lambda_{\mathrm{ex}}=360 \mathrm{~nm}\right)$ for benzopyrene

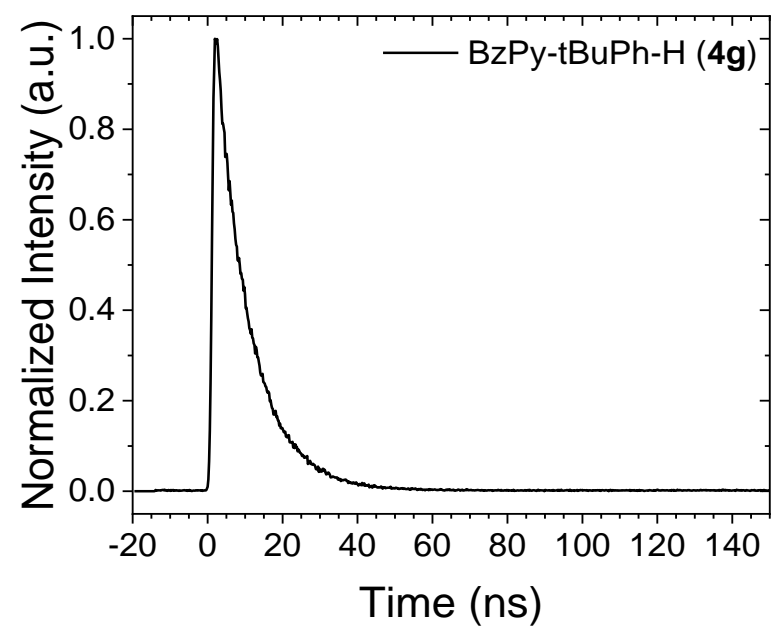




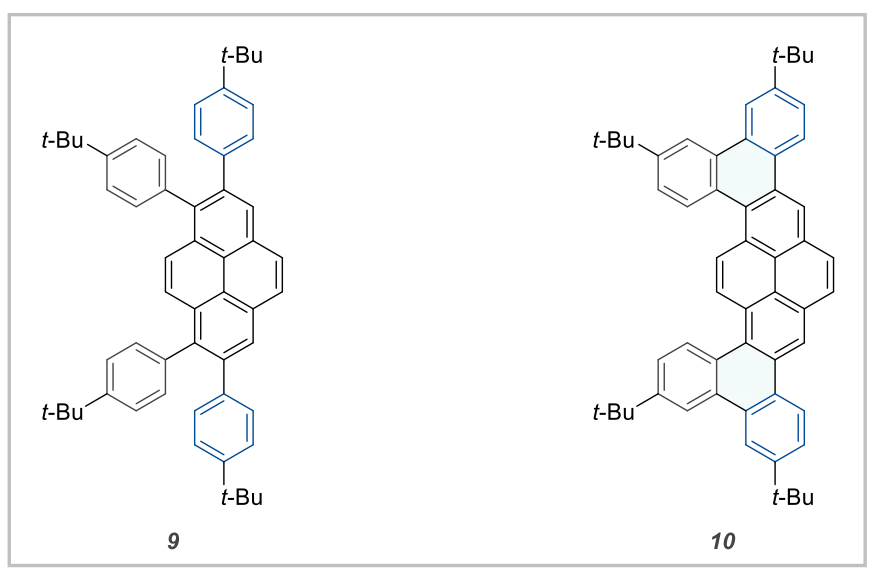

Room temperature time-resolved emission $\left(\lambda_{\mathrm{ex}}=360 \mathrm{~nm}\right)$ for 9

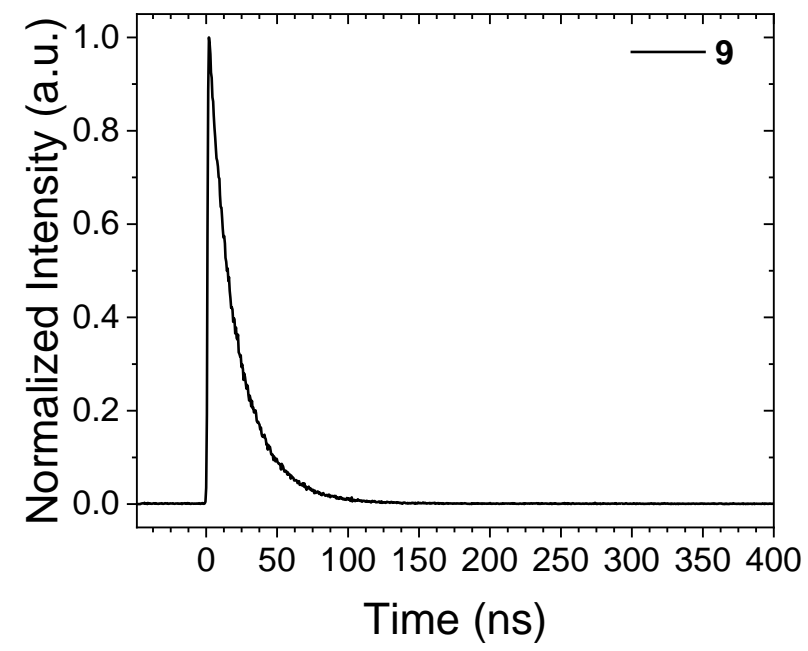


Room temperature absorption and emission overlay $9\left(\lambda_{\mathrm{ex}}=360 \mathrm{~nm}\right)$

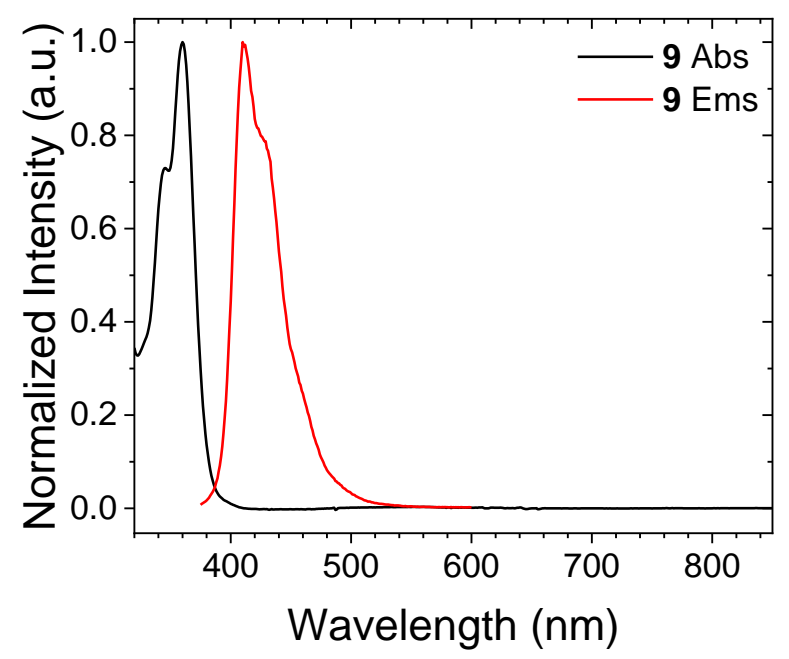

Absorption Normalized to Molar Extinction Coefficient (all spectra in $\mathrm{CH}_{2} \mathrm{Cl}_{2}$ )

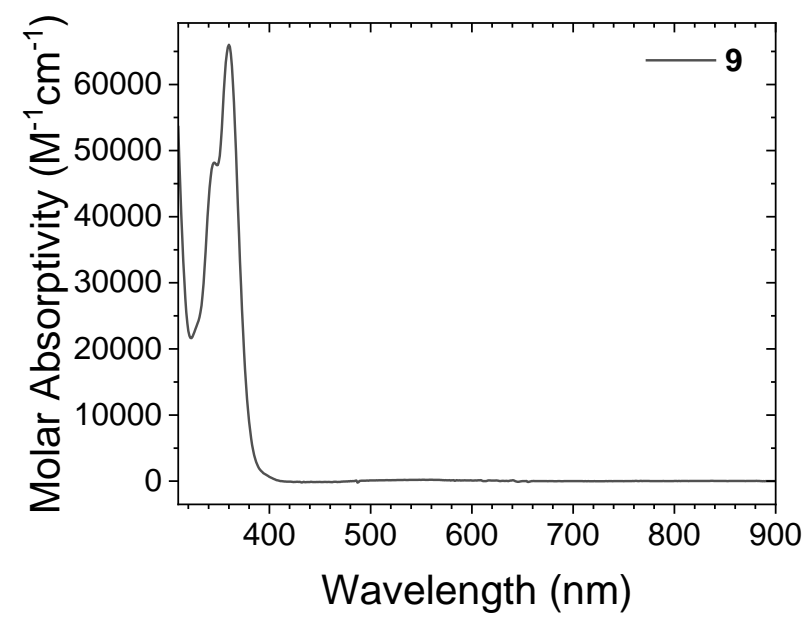


Room temperature absorption and emission $\left(\lambda_{\mathrm{ex}}=340 \mathrm{~nm}\right)$ for 10

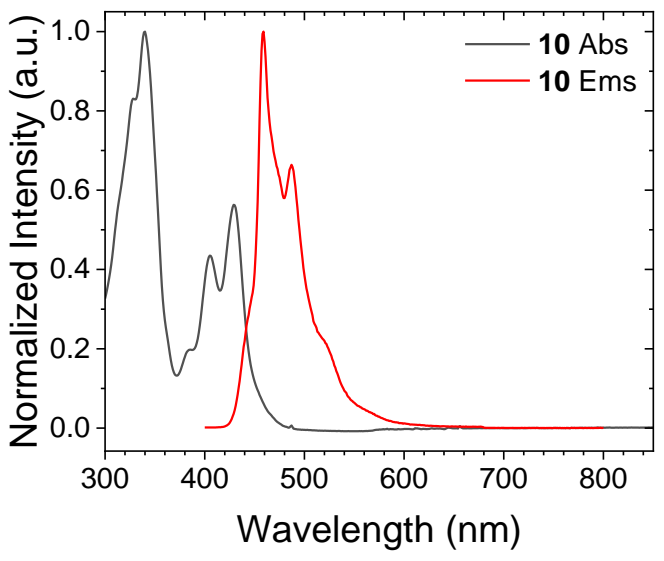

Room temperature time-resolved emission $\left(\lambda_{\text {ex }}=360 \mathrm{~nm}\right)$ for 10

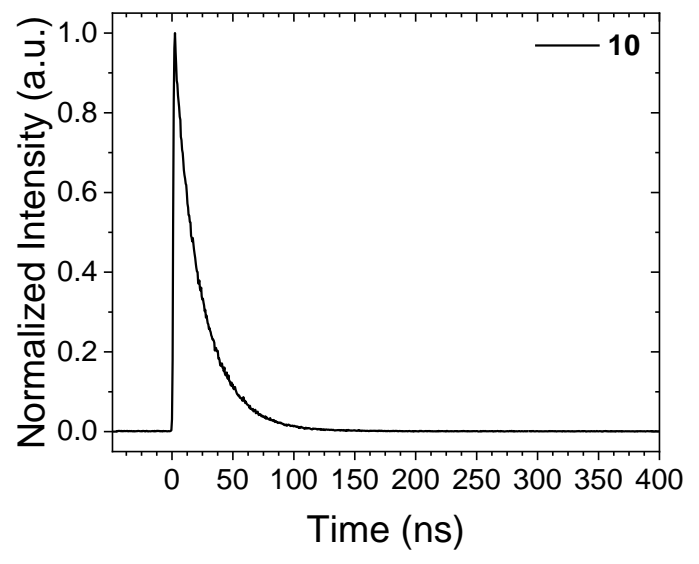




\section{Crystallographic Data}

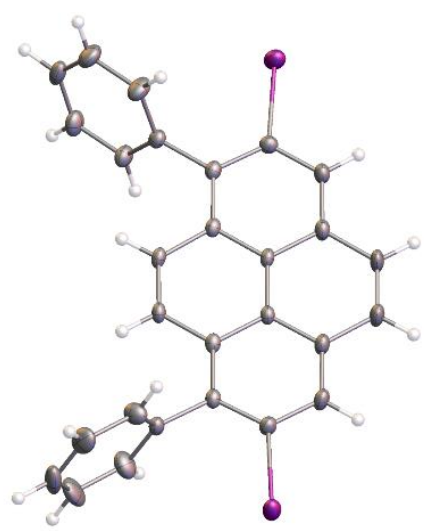

Figure S14. Single crystal X-ray structure diagram of $\mathbf{2 b}$. Displayed is the asymmetric unit with thermal ellipsoids at the 50\% probability level. Gray (C), Purple (I), White (H).

Table S2. Crystal data and structure refinement for $\mathbf{2 b}$.

Identification code

CCDC 1961765

Empirical formula

$\mathrm{C}_{28} \mathrm{H}_{16} \mathrm{I}_{2}+$ solvent

Formula weight

606.21

Temperature/K

127.43

Crystal system

monoclinic

Space group

$P 21 / n$

$a / \AA$

$8.8815(5)$

b/

23.1966(12)

$c / \AA$

22.1825(11)

$\alpha /^{\circ}$

90

$\beta /{ }^{\circ}$

98.8243(10)

$\gamma /{ }^{\circ}$

90

Volume $/ \AA^{3}$

4516.0(4)

$\mathrm{Z}$

8

$\rho_{\text {calc }} / \mathrm{cm}^{3}$

1.783

$\mu / \mathrm{mm}^{-1}$

2.798

$\mathrm{F}(000)$

5168.0

Crystal size $/ \mathrm{mm}^{3}$

$0.9 \times 0.3 \times 0.1$

Radiation

$\operatorname{MoK} \alpha(\lambda=0.71073)$

$2 \Theta$ range for data collection/ ${ }^{\circ} 4.728$ to 66.466

Index ranges

Reflections collected

Independent reflections

Data/restraints/parameters
$-13 \leq \mathrm{h} \leq 13,-35 \leq \mathrm{k} \leq 35,-34 \leq 1 \leq 34$

97135

$17299\left[\mathrm{R}_{\text {int }}=0.0362, \mathrm{R}_{\text {sigma }}=0.0272\right]$

$17299 / 0 / 541$ 
Goodness-of-fit on $\mathrm{F}^{2} \quad 1.057$

Final $R$ indexes $[\mathrm{I}>=2 \sigma(\mathrm{I})] \quad \mathrm{R}_{1}=0.0308, \mathrm{wR}_{2}=0.0645$

Final $\mathrm{R}$ indexes [all data] $\quad \mathrm{R}_{1}=0.0446, \mathrm{wR}_{2}=0.0694$

Largest diff. peak/hole / e $\AA^{-3} 1.07 /-0.48$

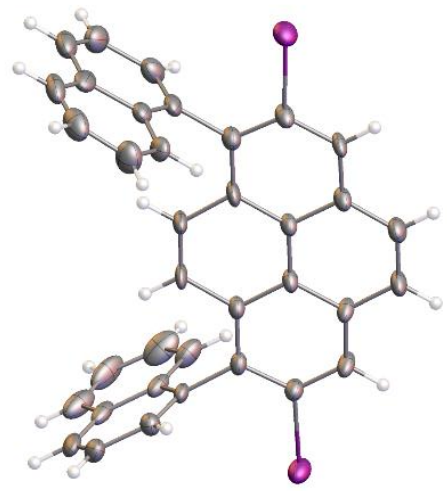

Figure S15. Single crystal X-ray structure of $\mathbf{2 c}$. Displayed is the asymmetric unit with thermal ellipsoids at the $50 \%$ probability level. Gray $(\mathrm{C})$, Purple $(\mathrm{I})$, and White $(\mathrm{H})$.

Table S3. Crystal data and structure refinement for $\mathbf{2 c}$.

Identification code

CCDC 1961764

Empirical formula

$\mathrm{C}_{36} \mathrm{H}_{20} \mathrm{I}_{2}+$ solvent

Formula weight

733.1

Temperature/K

129.25

Crystal system

monoclinic

Space group

$P 2{ }_{1} / n$

$a / \AA$

$18.184(2)$

$b / \AA$

9.1231(12)

$c / \AA$

18.269(2)

$\alpha /^{\circ}$

90

$\beta /{ }^{\circ}$

99.106(2)

$\gamma /{ }^{\circ}$

90

Volume $/ \AA^{3}$

2992.6(7)

$\mathrm{Z}$

$\rho_{\text {calcg }} / \mathrm{cm}^{3}$

4

$\mu / \mathrm{mm}^{-1}$

1.625

$\mathrm{F}(000)$

2.335

3428.0

Crystal size $/ \mathrm{mm}^{3}$

$0.1 \times 0.06 \times 0.02$

Radiation

$\operatorname{MoK} \alpha(\lambda=0.71073)$

$2 \Theta$ range for data collection $/{ }^{\circ} 4.516$ to 61.37

Index ranges

$$
-26 \leq \mathrm{h} \leq 26,-13 \leq \mathrm{k} \leq 13,-26 \leq 1 \leq 26
$$


Reflections collected $\quad 51696$

Independent reflections $\quad 9230\left[\mathrm{R}_{\text {int }}=0.0529, \mathrm{R}_{\text {sigma }}=0.0388\right]$

Data/restraints/parameters 9230/0/353

Goodness-of-fit on $\mathrm{F}^{2} \quad 1.121$

Final $\mathrm{R}$ indexes $[\mathrm{I}>=2 \sigma(\mathrm{I})] \quad \mathrm{R}_{1}=0.0753, \mathrm{wR}_{2}=0.1800$

Final $\mathrm{R}$ indexes [all data] $\quad \mathrm{R}_{1}=0.0974, \mathrm{wR}_{2}=0.1910$

Largest diff. peak/hole / e $\AA^{-3} 2.91 /-1.84$

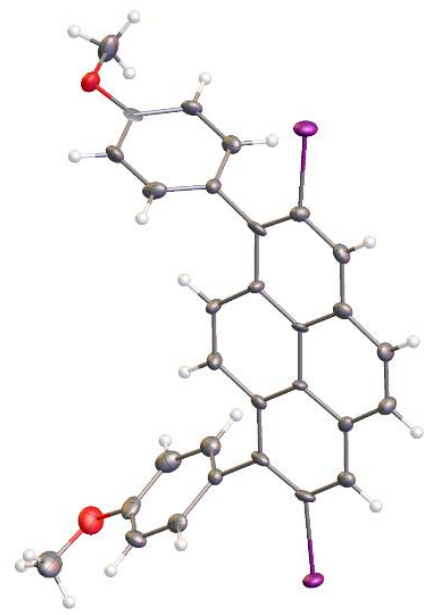

Figure S16. Single crystal X-ray structure diagram of $\mathbf{2 d}$. Displayed is the asymmetric unit with the $50 \%$ probability level ellipsoids. Gray (C), Red (O), Purple (I), White (H).

Table S4. Crystal data and structure refinement for $\mathbf{2 d}$.

Identification code

Empirical formula

Formula weight

Temperature/K

Crystal system

Space group

$a / \AA$

b/

$c / \AA$

$\alpha /{ }^{\circ}$

$\beta /{ }^{\circ}$

$\gamma /{ }^{\circ}$

Volume/ $\AA^{3}$

Z

$\rho_{\text {calc }} \mathrm{g} / \mathrm{cm}^{3}$

$\mu / \mathrm{mm}^{-1}$
CCDC 1961766

$\mathrm{C}_{180} \mathrm{H}_{126} \mathrm{O}_{12} \mathrm{I}_{12}$

3999.55

99.99

triclinic

$P-1$

14.4826(12)

$18.8530(15)$

26.938(2)

86.0590(10)

85.5660(10)

88.5040(10)

7314.2(10)

2

1.820

2.606 


$\begin{array}{ll}\mathrm{F}(000) & 7276.0 \\ \text { Crystal size/mm } & 0.6 \times 0.4 \times 0.1 \\ \text { Radiation } & \mathrm{MoK} \alpha(\lambda=0.71073) \\ 2 \Theta \text { range for data collection } /{ }^{\circ} & 4.302 \text { to } 58.556 \\ \text { Index ranges } & -19 \leq \mathrm{h} \leq 19,-25 \leq \mathrm{k} \leq 25,-37 \leq 1 \leq 37 \\ \text { Reflections collected } & 380081 \\ \text { Independent reflections } & 39760\left[\mathrm{R}_{\text {int }}=0.1602, \mathrm{R}_{\text {sigma }}=0.0916\right] \\ \text { Data/restraints/parameters } & 39760 / 0 / 1848 \\ \text { Goodness-of-fit on } \mathrm{F}^{2} & 1.113 \\ \text { Final R indexes }[\mathrm{I}>=2 \sigma(\mathrm{I})] & \mathrm{R}_{1}=0.0853, \mathrm{wR}_{2}=0.1504 \\ \text { Final R indexes [all data] } & \mathrm{R}_{1}=0.1569, \mathrm{wR}_{2}=0.1732 \\ \text { Largest diff. peak/hole / e } \AA^{-3} 3.51 /-1.36\end{array}$

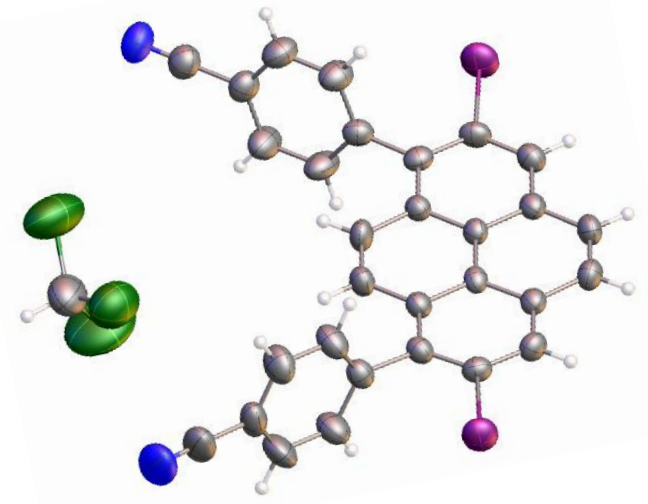

Figure S17. Single crystal X-ray structure of 2e. Displayed is the asymmetric unit with the thermal ellipsoids at the $50 \%$ probability level. Gray (C), Blue (N), Green (Cl), Purple (I), White (H).

Table S5. Crystal data and structure refinement for $\mathbf{2 e .}$

Identification code

Empirical formula

Formula weight

Temperature/K

Crystal system

Space group

$a / \AA$

$b / \AA$

$c / \AA$

$\alpha /{ }^{\circ}$

$\beta /{ }^{\circ}$

$\gamma /{ }^{\circ}$
CCDC 1961767

$\mathrm{C}_{31} \mathrm{H}_{16} \mathrm{Cl}_{3} \mathrm{I}_{2} \mathrm{~N}_{2}$

776.61

273.15

monoclinic

$P 21 / c$

$12.8520(14)$

20.361(2)

11.4010(12)

90

108.468(2)

90 


$\begin{array}{ll}\text { Volume} \AA^{3} & 2829.7(5) \\ \mathrm{Z} & 4 \\ \rho_{\text {calcg/cm }}{ }^{3} & 1.823 \\ \mu / \mathrm{mm}^{-1} & 2.530 \\ \mathrm{~F}(000) & 1492.0 \\ \mathrm{Crystal} \text { size } / \mathrm{mm}^{3} & 0.85 \times 0.64 \times 0.44 \\ \text { Radiation } & \mathrm{MoK} \alpha(\lambda=0.71073) \\ 2 \Theta \text { range for data collection/ } & 4.264 \text { to } 54.056 \\ \text { Index ranges } & -16 \leq \mathrm{h} \leq 16,-25 \leq \mathrm{k} \leq 26,-14 \leq 1 \leq 14 \\ \text { Reflections collected } & 77836 \\ \text { Independent reflections } & 6107\left[\mathrm{R}_{\text {int }}=0.0484, \mathrm{R}_{\text {sigma }}=0.0211\right] \\ \text { Data/restraints/parameters } & 6107 / 0 / 343 \\ \text { Goodness-of-fit on } \mathrm{F}^{2} & 1.092 \\ \text { Final R indexes [I }>=2 \sigma(\mathrm{I})] & \mathrm{R}_{1}=0.0440, \mathrm{wR}_{2}=0.0971 \\ \text { Final R indexes [all data] } & \mathrm{R}_{1}=0.0662, \mathrm{wR}_{2}=0.1128 \\ \text { Largest diff. peak/hole / e } \AA^{-3} & 1.06 /-0.72\end{array}$

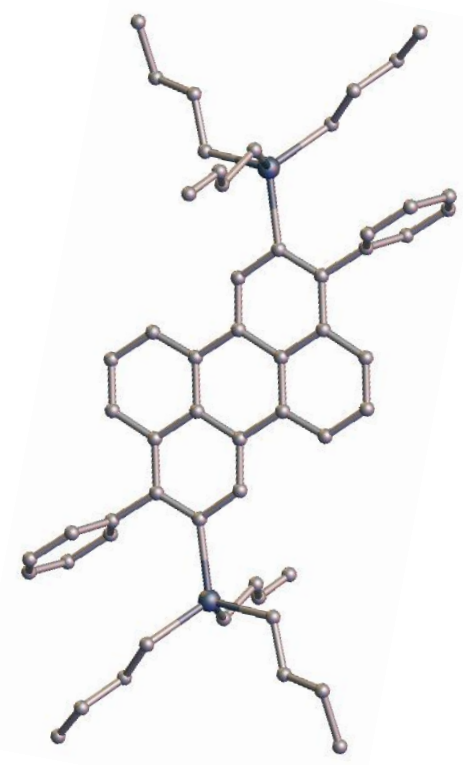

Figure S18. Single crystal X-ray structure of $\mathbf{2} \mathbf{f}^{\prime}$. Displayed is the asymmetric unit without hydrogens and thermal ellipsoids for clarity due to vibration disorder. Gray (C), Navy (Sn).

Table S6. Crystal data and structure refinement for $\mathbf{2 f}$ '.

Identification code CCDC 1961762

Empirical formula $\mathrm{C}_{56} \mathrm{H}_{68} \mathrm{Sn}_{2}$

Formula weight 978.52 


\begin{tabular}{|c|c|}
\hline Temperature/K & 298.01 \\
\hline Crystal system & triclinic \\
\hline Space group & $P-1$ \\
\hline$a / \AA$ & $10.0922(6)$ \\
\hline$b / \AA$ & $11.2174(6)$ \\
\hline$c / \AA$ & $12.9069(7)$ \\
\hline$\alpha /{ }^{\circ}$ & $90.7726(14)$ \\
\hline$\beta /{ }^{\circ}$ & $102.0131(14)$ \\
\hline$\gamma /{ }^{\circ}$ & $114.0745(13)$ \\
\hline Volume $/ \AA^{3}$ & $1296.91(13)$ \\
\hline $\mathrm{Z}$ & 1 \\
\hline$\rho_{\text {calc }} g / \mathrm{cm}^{3}$ & 1.253 \\
\hline$\mu / \mathrm{mm}^{-1}$ & 0.995 \\
\hline $\mathrm{F}(000)$ & 504.0 \\
\hline Crystal size $/ \mathrm{mm}^{3}$ & $0.72 \times 0.48 \times 0.17$ \\
\hline Radiation & $\operatorname{MoK} \alpha(\lambda=0.71073)$ \\
\hline \multicolumn{2}{|c|}{$2 \Theta$ range for data collection $/{ }^{\circ} 4.546$ to 55.092} \\
\hline Index ranges & $-13 \leq \mathrm{h} \leq 13,-14 \leq \mathrm{k} \leq 14,-16 \leq 1 \leq 16$ \\
\hline Reflections collected & 20016 \\
\hline Independent reflections & $5958\left[\mathrm{R}_{\text {int }}=0.0524, \mathrm{R}_{\text {sigma }}=0.0622\right]$ \\
\hline Data/restraints/parameters & $5958 / 0 / 265$ \\
\hline Goodness-of-fit on $\mathrm{F}^{2}$ & 0.973 \\
\hline Final $R$ indexes $[\mathrm{I}>=2 \sigma(\mathrm{I})]$ & $\mathrm{R}_{1}=0.0646, \mathrm{wR}_{2}=0.1485$ \\
\hline Final $\mathrm{R}$ indexes [all data] & $\mathrm{R}_{1}=0.1558, \mathrm{wR}_{2}=0.1881$ \\
\hline Largest diff. peak/hole / e $\AA^{-}$ & $0.79 /-0.25$ \\
\hline
\end{tabular}

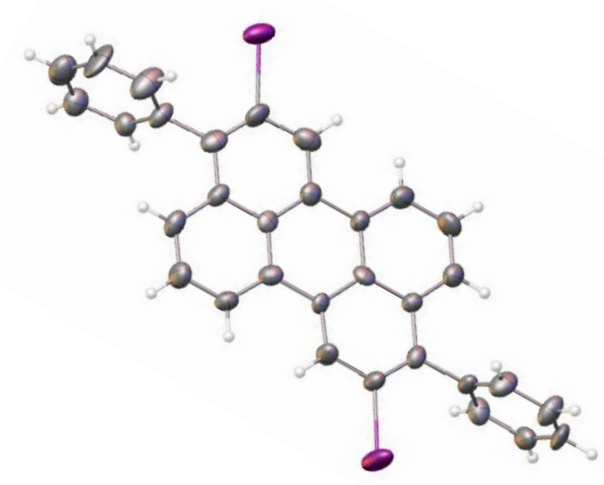

Figure S19. Single crystal X-ray structure diagram of $\mathbf{3} \mathbf{f}^{\prime}$. Displayed is the asymmetric unit with thermal ellipsoids at the 50\% probability level. Gray (C), Purple (I), White (H). 
Table S7. Crystal data and structure refinement for 3f'.

Identification code

CCDC 1961763

Empirical formula

$\mathrm{C}_{32} \mathrm{H}_{18} \mathrm{I}_{2}$

Formula weight

656.26

Temperature/K

273.15

Crystal system

monoclinic

Space group

Cc

$a / \AA$

$10.5455(10)$

b/A

28.067(3)

$c / \AA$

$9.2868(9)$

$\alpha /^{\circ}$

90

$\beta /{ }^{\circ}$

$116.926(3)$

$\gamma /{ }^{\circ}$

90

Volume $/ \AA^{3}$

2450.7(4)

$\mathrm{Z}$

4

$\rho_{\text {calc }} \mathrm{g} / \mathrm{cm}^{3}$

1.779

$\mu / \mathrm{mm}^{-1}$

2.585

$\mathrm{F}(000)$

1264.0

Crystal size $/ \mathrm{mm}^{3}$

$0.62 \times 0.54 \times 0.13$

Radiation

$\operatorname{MoK} \alpha(\lambda=0.71073)$

$2 \Theta$ range for data collection $/{ }^{\circ} 5.078$ to 61.298

Index ranges

$-15 \leq \mathrm{h} \leq 15,-40 \leq \mathrm{k} \leq 40,-13 \leq 1 \leq 13$

Reflections collected 22703

Independent reflections

$7523\left[\mathrm{R}_{\text {int }}=0.0816, \mathrm{R}_{\text {sigma }}=0.1247\right]$

Data/restraints/parameters

$7523 / 2 / 307$

Goodness-of-fit on $\mathrm{F}^{2}$

1.065

Final $R$ indexes $[\mathrm{I}>=2 \sigma(\mathrm{I})] \quad \mathrm{R}_{1}=0.0852, \mathrm{wR}_{2}=0.1219$

Final $\mathrm{R}$ indexes [all data] $\quad \mathrm{R}_{1}=0.1832, \mathrm{wR}_{2}=0.1434$

Largest diff. peak/hole / e $\AA^{-3} 1.08 /-0.48$

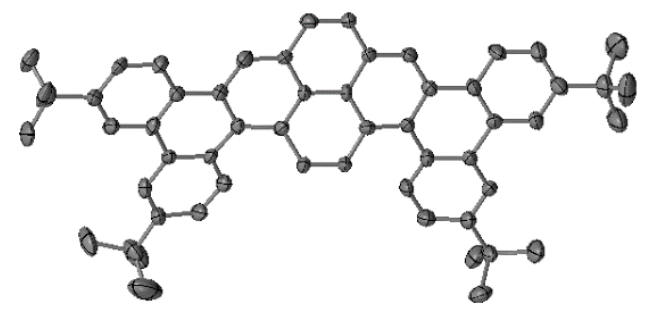

Figure S20. Single crystal X-ray structure diagram of 10. Displayed is the asymmetric unit with thermal ellipsoids at the $50 \%$ probability level. Gray (C). 
Table 8. Crystal data and structure refinement for $\mathbf{1 0 .}$

Identification code

Empirical formula

Formula weight

Temperature/K

Crystal system

Space group

$\mathrm{a} / \AA$

$\mathrm{b} / \AA$

$\mathrm{c} / \AA$

$\alpha /{ }^{\circ}$

$\beta /{ }^{\circ}$

$\gamma /{ }^{\circ}$

Volume $/ \AA^{3}$

$\mathrm{Z}$

$\rho_{\text {calc }} / \mathrm{cm}^{3}$

$\mu / \mathrm{mm}^{-1}$

$\mathrm{F}(000)$

Crystal size $/ \mathrm{mm}^{3}$

Radiation

$2 \Theta$ range for data collection ${ }^{\circ}$

Index ranges

Reflections collected

Independent reflections

Data/restraints/parameters

Goodness-of-fit on $\mathrm{F}^{2}$

Final $\mathrm{R}$ indexes $[\mathrm{I}>=2 \sigma(\mathrm{I})]$

Final $\mathrm{R}$ indexes [all data]

Largest diff. peak/hole / e $\AA^{-3}$

Flack parameter
20200115-1

$\mathrm{C}_{56} \mathrm{H}_{54}$

726.99

150.00(10)

orthorhombic

Pca2 1

28.313(2)

18.0423(9)

$8.0929(4)$

90

90

90

4134.2(4)

4

1.168

0.491

1560.0

$0.234 \times 0.026 \times 0.024$

$\mathrm{Cu} \mathrm{K \alpha}(\lambda=1.54184)$

5.808 to 157.708

$-36 \leq \mathrm{h} \leq 35,-21 \leq \mathrm{k} \leq 22,-9 \leq 1 \leq 9$

23739

$6876\left[\mathrm{R}_{\text {int }}=0.0662, \mathrm{R}_{\text {sigma }}=0.0627\right]$

$6876 / 1 / 517$

1.465

$\mathrm{R}_{1}=0.1389, \mathrm{wR}_{2}=0.3553$

$\mathrm{R}_{1}=0.1530, \mathrm{wR}_{2}=0.3662$

$0.64 /-0.39$

$-0.1(10)$ 


\section{Computational Details}

DFT calculations were carried with the Gaussian 09 software package, ${ }^{4}$ using the (U)M06-2X DFT functional ${ }^{5,6}$ with the LanL2DZ basis set for all atoms. A broken-spin approach was used when necessary. Frequency calculations were conducted for all structures, confirming if a structure is either a minimum or a TS. Natural Bond Orbital ${ }^{7-11}$ (NBO) analysis was performed on key structures. The Gibbs Free energy values are reported at $298 \mathrm{~K}$, unless otherwise noted. Larger geometries were pre-optimized with Grimme's $x t b,{ }^{12}$ at the GFN2-xTB level of theory. Conformer generation and evaluation was performed with crest, ${ }^{13}$ at the at the GFN2-xTB level of theory. Time-dependent DFT (at the $(S M D=D C M) / U M 06-2 X / 6-311++G^{* *}$ level of theory) calculations were performed on the conformers generated with crest to ensure a reasonable sampling of structures. Electron Density of Delocalized Bonds (EDDB) ${ }^{14}$ calculations were performed for selected polyarenes for a description of their electronic delocalization and conjugation. These calculations were performed at the M062X/cc-pvtz level of theory, with NBO6.

Three-dimensional structures and orbital plots were produced with CYLView $1.0 .1,{ }^{15}$ UCSF Chimera, ${ }^{16}$ and Chemcraft $1.7^{17}$. Geometries, energies and frequencies presented in this SI were organized with ESIgen. ${ }^{18}$

Structures and energies for all species calculated at the UM06-2X/LanL2DZ level of theory

Calculated geometries (in cartesian coordinates) with their respective energies (in hartree), charge, multiplicity and stoichiometry.

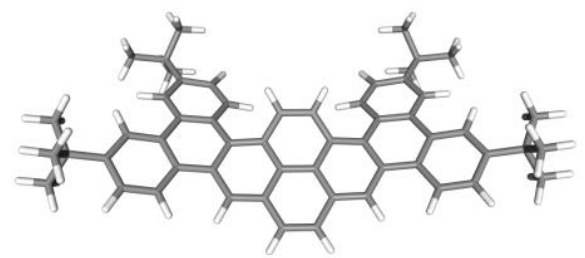

Charge

0

Multiplicity

Stoichiometry

Number of Basis Functions

Electronic Energy (Eh)

Mean of alpha and beta Electrons

1

C56H54

1610

$-2166.28719476$

195

Molecular Geometry in Cartesian Coordinates

$\begin{array}{llll}\mathrm{C} & 9.959920 & -2.619348 & -0.186445 \\ \mathrm{H} & 9.908052 & -1.932929 & -1.029626 \\ \mathrm{H} & 9.616752 & -3.594802 & -0.520286 \\ \mathrm{H} & 10.999738 & -2.711503 & 0.120718 \\ \mathrm{C} & 9.109549 & -2.093248 & 0.979596 \\ \mathrm{C} & 7.667635 & -1.953062 & 0.516664 \\ \mathrm{C} & 6.984556 & -0.754027 & 0.526518 \\ \mathrm{C} & 5.655783 & -0.633986 & 0.100876\end{array}$




\begin{tabular}{|c|c|c|c|}
\hline $\mathrm{C}$ & 4.955159 & -1.790044 & -0.298469 \\
\hline $\mathrm{C}$ & 5.661357 & -2.998700 & -0.327461 \\
\hline $\mathrm{C}$ & 6.977707 & -3.078243 & 0.055159 \\
\hline $\mathrm{H}$ & 7.469313 & -4.038725 & 0.015050 \\
\hline $\mathrm{H}$ & 5.167287 & -3.906903 & -0.634921 \\
\hline $\mathrm{C}$ & 3.527401 & -1.726963 & -0.553676 \\
\hline $\mathrm{C}$ & 2.797138 & -2.838484 & -0.980970 \\
\hline $\mathrm{C}$ & 1.414729 & -2.815959 & -1.088986 \\
\hline $\mathrm{C}$ & 0.707569 & -1.672824 & -0.632607 \\
\hline $\mathrm{C}$ & 1.427093 & -0.544134 & -0.179790 \\
\hline $\mathrm{C}$ & 2.841234 & -0.520463 & -0.294941 \\
\hline $\mathrm{C}$ & 3.605398 & 0.701812 & -0.214232 \\
\hline $\mathrm{C}$ & 3.027756 & 1.949767 & -0.488041 \\
\hline $\mathrm{C}$ & 3.760220 & 3.109620 & -0.435081 \\
\hline $\mathrm{C}$ & 5.124220 & 3.090925 & -0.126764 \\
\hline $\mathrm{C}$ & 5.714929 & 1.856530 & 0.057727 \\
\hline $\mathrm{C}$ & 4.996293 & 0.656048 & 0.008521 \\
\hline $\mathrm{H}$ & 6.777831 & 1.807364 & 0.212638 \\
\hline $\mathrm{C}$ & 5.896668 & 4.399188 & -0.057690 \\
\hline $\mathrm{C}$ & 7.366896 & 4.201927 & 0.324806 \\
\hline $\mathrm{H}$ & 7.893053 & 3.615599 & -0.425400 \\
\hline $\mathrm{H}$ & 7.456917 & 3.711689 & 1.291839 \\
\hline $\mathrm{H}$ & 7.855857 & 5.171855 & 0.393080 \\
\hline $\mathrm{C}$ & 5.845608 & 5.084207 & -1.431614 \\
\hline $\mathrm{H}$ & 4.824166 & 5.315973 & -1.721060 \\
\hline $\mathrm{H}$ & 6.412291 & 6.012857 & -1.408258 \\
\hline $\mathrm{H}$ & 6.276140 & 4.435319 & -2.192184 \\
\hline $\mathrm{C}$ & 5.246771 & 5.304613 & 0.999515 \\
\hline $\mathrm{H}$ & 5.265757 & 4.819115 & 1.973607 \\
\hline $\mathrm{H}$ & 4.212907 & 5.527595 & 0.750496 \\
\hline $\mathrm{H}$ & 5.789459 & 6.245014 & 1.071627 \\
\hline $\mathrm{H}$ & 3.266266 & 4.042894 & -0.660519 \\
\hline $\mathrm{H}$ & 1.995249 & 1.998130 & -0.798647 \\
\hline $\mathrm{C}$ & 0.679310 & 0.460896 & 0.493037 \\
\hline $\mathrm{C}$ & -0.679221 & 0.460875 & 0.493257 \\
\hline $\mathrm{C}$ & -1.427199 & -0.544066 & -0.179506 \\
\hline $\mathrm{C}$ & -0.707794 & -1.672766 & -0.632498 \\
\hline $\mathrm{C}$ & -1.415082 & -2.815849 & -1.088779 \\
\hline $\mathrm{C}$ & -2.797493 & -2.838197 & -0.980846 \\
\hline $\mathrm{C}$ & -3.527687 & -1.726555 & -0.553724 \\
\hline $\mathrm{C}$ & -2.841354 & -0.520249 & -0.294627 \\
\hline $\mathrm{C}$ & -3.605358 & 0.702067 & -0.213194 \\
\hline $\mathrm{C}$ & -3.027439 & 1.950031 & -0.486374 \\
\hline $\mathrm{C}$ & -3.759337 & 3.110138 & -0.431677 \\
\hline $\mathrm{C}$ & -5.123023 & 3.091695 & -0.121953 \\
\hline $\mathrm{C}$ & -5.714182 & 1.857339 & 0.061409 \\
\hline $\mathrm{C}$ & -4.996199 & 0.656517 & 0.010056 \\
\hline
\end{tabular}




\begin{tabular}{|c|c|c|c|}
\hline $\mathrm{C}$ & -5.656162 & -0.633429 & 0.100698 \\
\hline $\mathrm{C}$ & -6.985404 & -0.753562 & 0.524972 \\
\hline $\mathrm{C}$ & -7.668832 & -1.952387 & 0.513265 \\
\hline $\mathrm{C}$ & -6.978847 & -3.077303 & 0.051217 \\
\hline $\mathrm{C}$ & -5.662176 & -2.997728 & -0.330202 \\
\hline $\mathrm{C}$ & -4.955607 & -1.789332 & -0.299323 \\
\hline $\mathrm{H}$ & -5.168227 & -3.905754 & -0.638346 \\
\hline $\mathrm{H}$ & -7.470716 & -4.037581 & 0.009586 \\
\hline $\mathrm{C}$ & -9.111256 & -2.092800 & 0.974541 \\
\hline $\mathrm{C}$ & -9.165698 & -3.086487 & 2.144670 \\
\hline $\mathrm{H}$ & -8.549060 & -2.731903 & 2.968623 \\
\hline $\mathrm{H}$ & -8.805533 & -4.068042 & 1.848620 \\
\hline $\mathrm{H}$ & -10.189043 & -3.191924 & 2.499256 \\
\hline $\mathrm{C}$ & -9.716095 & -0.766761 & 1.446105 \\
\hline $\mathrm{H}$ & -9.163630 & -0.362694 & 2.291631 \\
\hline $\mathrm{H}$ & -9.728820 & -0.034107 & 0.642024 \\
\hline $\mathrm{H}$ & -10.743496 & -0.931476 & 1.765215 \\
\hline $\mathrm{C}$ & -9.960561 & -2.617019 & -0.193133 \\
\hline $\mathrm{H}$ & -9.907722 & -1.929376 & -1.035253 \\
\hline $\mathrm{H}$ & -11.000702 & -2.709452 & 0.112850 \\
\hline $\mathrm{H}$ & -9.617218 & -3.592039 & -0.528057 \\
\hline $\mathrm{H}$ & -7.482837 & 0.127657 & 0.889190 \\
\hline $\mathrm{H}$ & -6.776954 & 1.808585 & 0.217352 \\
\hline $\mathrm{C}$ & -5.894481 & 4.400377 & -0.049921 \\
\hline $\mathrm{C}$ & -7.364584 & 4.203515 & 0.333214 \\
\hline $\mathrm{H}$ & -7.852838 & 5.173675 & 0.403260 \\
\hline $\mathrm{H}$ & -7.454304 & 3.711900 & 1.299574 \\
\hline $\mathrm{H}$ & -7.891624 & 3.618663 & -0.417524 \\
\hline $\mathrm{C}$ & -5.243084 & 5.303175 & 1.008619 \\
\hline $\mathrm{H}$ & -5.262227 & 4.815971 & 1.981855 \\
\hline $\mathrm{H}$ & -4.209054 & 5.525347 & 0.759560 \\
\hline $\mathrm{H}$ & -5.784616 & 6.244096 & 1.082624 \\
\hline $\mathrm{C}$ & -5.843844 & 5.088070 & -1.422523 \\
\hline $\mathrm{H}$ & -4.822463 & 5.319943 & -1.712088 \\
\hline $\mathrm{H}$ & -6.410063 & 6.016947 & -1.397029 \\
\hline $\mathrm{H}$ & -6.275104 & 4.440881 & -2.184124 \\
\hline $\mathrm{H}$ & -3.265180 & 4.043421 & -0.656626 \\
\hline $\mathrm{H}$ & -1.995165 & 1.998222 & -0.797779 \\
\hline $\mathrm{H}$ & -3.305650 & -3.748132 & -1.259679 \\
\hline $\mathrm{C}$ & -0.675931 & -3.932629 & -1.584529 \\
\hline $\mathrm{C}$ & 0.675426 & -3.932661 & -1.584685 \\
\hline $\mathrm{H}$ & 1.228777 & -4.787884 & -1.947602 \\
\hline $\mathrm{H}$ & -1.229409 & -4.787836 & -1.947290 \\
\hline $\mathrm{H}$ & -1.210398 & 1.200624 & 1.073759 \\
\hline $\mathrm{H}$ & 1.210667 & 1.200684 & 1.073330 \\
\hline $\mathrm{H}$ & 3.305307 & -3.748408 & -1.259854 \\
\hline $\mathrm{H}$ & 7.481753 & 0.127428 & 0.890472 \\
\hline
\end{tabular}




$\begin{array}{cccc}\mathrm{C} & 9.714296 & -0.766711 & 1.449903 \\ \mathrm{H} & 10.741426 & -0.931176 & 1.770014 \\ \mathrm{H} & 9.727760 & -0.035136 & 0.644855 \\ \mathrm{H} & 9.161262 & -0.361410 & 2.294466 \\ \mathrm{C} & 9.162503 & -3.085251 & 2.151211 \\ \mathrm{H} & 8.545220 & -2.729241 & 2.974068 \\ \mathrm{H} & 10.185470 & -3.190578 & 2.506923 \\ \mathrm{H} & 8.802215 & -4.067076 & 1.856211\end{array}$

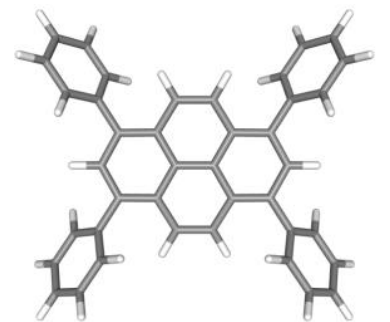

Charge

Multiplicity

Stoichiometry

Number of Basis Functions

Electronic Energy (Eh)

Mean of alpha and beta Electrons
0

1

$\mathrm{C} 40 \mathrm{H} 26$

1062

$-1539.76582196$

133

Molecular Geometry in Cartesian Coordinates

$\begin{array}{lrrr}\mathrm{C} & 3.495537 & 0.000017 & -0.000383 \\ \mathrm{C} & 2.825477 & 1.213823 & -0.055844 \\ \mathrm{C} & 1.419305 & 1.225740 & -0.097689 \\ \mathrm{C} & 0.710933 & -0.000264 & -0.002353 \\ \mathrm{C} & 1.419389 & -1.226180 & 0.093337 \\ \mathrm{C} & 2.825613 & -1.213891 & 0.053727 \\ \mathrm{C} & 3.620602 & -2.454627 & 0.060709 \\ \mathrm{C} & 4.637151 & -2.631907 & 0.995918 \\ \mathrm{C} & 5.408345 & -3.780030 & 0.987676 \\ \mathrm{C} & 5.182155 & -4.763981 & 0.039274 \\ \mathrm{C} & 4.181301 & -4.591902 & -0.903034 \\ \mathrm{C} & 3.405122 & -3.447236 & -0.893116 \\ \mathrm{H} & 2.635385 & -3.308825 & -1.639220 \\ \mathrm{H} & 4.005748 & -5.352566 & -1.650424 \\ \mathrm{H} & 5.785807 & -5.659955 & 0.032089 \\ \mathrm{H} & 6.189195 & -3.907648 & 1.723891 \\ \mathrm{H} & 4.810524 & -1.868762 & 1.741559 \\ \mathrm{C} & 0.676801 & -2.426617 & 0.281536 \\ \mathrm{C} & -0.676407 & -2.426573 & 0.282451 \\ \mathrm{C} & -1.419196 & -1.226203 & 0.094494 \\ \mathrm{C} & -2.825454 & -1.213940 & 0.055863\end{array}$




\begin{tabular}{|c|c|c|c|}
\hline $\mathrm{C}$ & -3.495374 & -0.000105 & -0.000217 \\
\hline $\mathrm{C}$ & -2.825385 & 1.213634 & -0.057609 \\
\hline $\mathrm{C}$ & -1.419201 & 1.225634 & -0.098535 \\
\hline $\mathrm{C}$ & -0.710819 & -0.000302 & -0.002271 \\
\hline $\mathrm{C}$ & -0.676526 & 2.425814 & -0.288130 \\
\hline $\mathrm{C}$ & 0.676666 & 2.425908 & -0.287437 \\
\hline $\mathrm{H}$ & 1.218691 & 3.345432 & -0.451789 \\
\hline $\mathrm{H}$ & -1.218488 & 3.345253 & -0.453113 \\
\hline $\mathrm{C}$ & -3.620221 & 2.454427 & -0.066076 \\
\hline $\mathrm{C}$ & -3.402693 & 3.449630 & 0.884579 \\
\hline $\mathrm{C}$ & -4.178353 & 4.594664 & 0.892583 \\
\hline $\mathrm{C}$ & -5.180972 & 4.764375 & -0.048277 \\
\hline $\mathrm{C}$ & -5.409441 & 3.777696 & -0.993299 \\
\hline $\mathrm{C}$ & -4.638591 & 2.629338 & -0.999771 \\
\hline $\mathrm{H}$ & -4.813534 & 1.864176 & -1.742976 \\
\hline $\mathrm{H}$ & -6.191733 & 3.903412 & -1.728313 \\
\hline $\mathrm{H}$ & -5.784265 & 5.660600 & -0.042536 \\
\hline $\mathrm{H}$ & -4.001128 & 5.357405 & 1.637461 \\
\hline $\mathrm{H}$ & -2.631792 & 3.312949 & 1.629796 \\
\hline $\mathrm{H}$ & -4.576761 & -0.000027 & 0.000868 \\
\hline $\mathrm{C}$ & -3.620640 & -2.454518 & 0.066182 \\
\hline $\mathrm{C}$ & -3.405836 & -3.449800 & -0.884998 \\
\hline $\mathrm{C}$ & -4.181948 & -4.594548 & -0.891049 \\
\hline $\mathrm{C}$ & -5.182213 & -4.763935 & 0.052372 \\
\hline $\mathrm{C}$ & -5.407963 & -3.777172 & 0.997953 \\
\hline $\mathrm{C}$ & -4.636722 & -2.629071 & 1.002439 \\
\hline $\mathrm{H}$ & -4.809664 & -1.863788 & 1.745987 \\
\hline $\mathrm{H}$ & -6.188401 & -3.902629 & 1.734980 \\
\hline $\mathrm{H}$ & -5.785762 & -5.659998 & 0.048236 \\
\hline $\mathrm{H}$ & -4.006835 & -5.357395 & -1.636318 \\
\hline $\mathrm{H}$ & -2.636730 & -3.313391 & -1.632127 \\
\hline $\mathrm{H}$ & -1.218271 & -3.346222 & 0.446561 \\
\hline $\mathrm{H}$ & 1.218890 & -3.346307 & 0.444739 \\
\hline $\mathrm{C}$ & 3.620094 & 2.454747 & -0.060787 \\
\hline $\mathrm{C}$ & 4.640286 & 2.631686 & -0.992103 \\
\hline $\mathrm{C}$ & 5.411213 & 3.779968 & -0.981519 \\
\hline $\mathrm{C}$ & 5.180986 & 4.764551 & -0.034736 \\
\hline $\mathrm{C}$ & 4.176378 & 4.592903 & 0.903650 \\
\hline $\mathrm{C}$ & 3.400660 & 3.447959 & 0.891536 \\
\hline $\mathrm{H}$ & 2.628247 & 3.309754 & 1.634894 \\
\hline $\mathrm{H}$ & 3.997677 & 5.354004 & 1.649852 \\
\hline $\mathrm{H}$ & 5.784373 & 5.660684 & -0.025759 \\
\hline $\mathrm{H}$ & 6.194961 & 3.907276 & -1.714704 \\
\hline $\mathrm{H}$ & 4.816596 & 1.868206 & -1.736714 \\
\hline $\mathrm{H}$ & 4.576924 & 0.000097 & 0.000796 \\
\hline
\end{tabular}




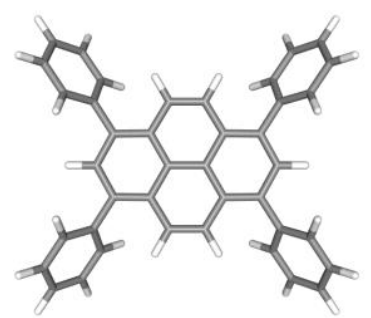

Charge

Multiplicity

Stoichiometry

Number of Basis Functions

Electronic Energy (Eh)

Mean of alpha and beta Electrons
0

1

C40H26

1062

$-1539.76534116$

133

Molecular Geometry in Cartesian Coordinates

$\begin{array}{lrrr}\mathrm{C} & -3.496412 & -0.000251 & -0.061600 \\ \mathrm{C} & -2.825830 & -1.214920 & -0.060069 \\ \mathrm{C} & -1.419619 & -1.229817 & -0.087961 \\ \mathrm{C} & -0.711123 & -0.000064 & -0.088184 \\ \mathrm{C} & -1.419786 & 1.229593 & -0.087491 \\ \mathrm{C} & -2.826008 & 1.214505 & -0.059952 \\ \mathrm{C} & -3.622323 & 2.453894 & -0.004457 \\ \mathrm{C} & -3.435413 & 3.381230 & 1.018132 \\ \mathrm{C} & -4.212075 & 4.523999 & 1.080151 \\ \mathrm{C} & -5.184175 & 4.759061 & 0.121555 \\ \mathrm{C} & -5.382353 & 3.839447 & -0.895164 \\ \mathrm{C} & -4.611280 & 2.692757 & -0.955088 \\ \mathrm{H} & -4.763208 & 1.979158 & -1.752623 \\ \mathrm{H} & -6.141149 & 4.016411 & -1.644099 \\ \mathrm{H} & -5.787927 & 5.653712 & 0.169227 \\ \mathrm{H} & -4.059254 & 5.233590 & 1.880851 \\ \mathrm{H} & -2.688177 & 3.191932 & 1.775875 \\ \mathrm{C} & -0.676894 & 2.441441 & -0.174817 \\ \mathrm{C} & 0.676194 & 2.441525 & -0.174797 \\ \mathrm{C} & 1.419259 & 1.229789 & -0.087306 \\ \mathrm{C} & 2.825485 & 1.214867 & -0.059347 \\ \mathrm{C} & 3.495989 & 0.000155 & -0.061119 \\ \mathrm{C} & 2.825590 & -1.214608 & -0.060108 \\ \mathrm{C} & 1.419388 & -1.229667 & -0.087992 \\ \mathrm{C} & 0.710766 & 0.000023 & -0.088128 \\ \mathrm{C} & 0.676494 & -2.441473 & -0.176022 \\ \mathrm{C} & -0.676591 & -2.441549 & -0.175952 \\ \mathrm{H} & -1.218442 & -3.371434 & -0.264789 \\ \mathrm{H} & 1.218438 & -3.371295 & -0.264925 \\ \mathrm{C} & 3.622089 & -2.453868 & -0.004647\end{array}$




$\begin{array}{lrrr}\mathrm{C} & 4.612015 & -2.691944 & -0.954472 \\ \mathrm{C} & 5.383651 & -3.838234 & -0.894189 \\ \mathrm{C} & 5.185039 & -4.758269 & 0.122067 \\ \mathrm{C} & 4.211867 & -4.524062 & 1.079785 \\ \mathrm{C} & 3.434674 & -3.381668 & 1.017427 \\ \mathrm{H} & 2.686674 & -3.192982 & 1.774566 \\ \mathrm{H} & 4.058704 & -5.233962 & 1.880144 \\ \mathrm{H} & 5.789262 & -5.652586 & 0.170039 \\ \mathrm{H} & 6.143222 & -4.014554 & -1.642493 \\ \mathrm{H} & 4.764347 & -1.978039 & -1.751655 \\ \mathrm{H} & 4.576800 & 0.000164 & -0.022232 \\ \mathrm{C} & 3.621787 & 2.454199 & -0.003275 \\ \mathrm{C} & 4.612448 & 2.692325 & -0.952328 \\ \mathrm{C} & 5.383638 & 3.838898 & -0.891758 \\ \mathrm{C} & 5.183780 & 4.759243 & 0.123970 \\ \mathrm{C} & 4.209973 & 4.524938 & 1.081019 \\ \mathrm{C} & 3.433321 & 3.382197 & 1.018443 \\ \mathrm{H} & 2.684897 & 3.193413 & 1.775133 \\ \mathrm{H} & 4.055871 & 5.235052 & 1.881006 \\ \mathrm{H} & 5.787559 & 5.653851 & 0.172095 \\ \mathrm{H} & 6.143774 & 4.015252 & -1.639476 \\ \mathrm{H} & 4.765623 & 1.978275 & -1.749219 \\ \mathrm{H} & 1.217973 & 3.371488 & -0.263170 \\ \mathrm{H} & -1.218815 & 3.371332 & -0.263127 \\ \mathrm{C} & -3.621914 & -2.454406 & -0.003772 \\ \mathrm{C} & -3.433890 & -3.381291 & 1.019040 \\ \mathrm{C} & -4.210364 & -4.524101 & 1.082374 \\ \mathrm{C} & -5.183409 & -4.759672 & 0.124855 \\ \mathrm{C} & -5.382656 & -3.840547 & -0.892094 \\ \mathrm{C} & -4.611761 & -2.693791 & -0.953324 \\ \mathrm{H} & -4.764595 & -1.980598 & -1.751050 \\ \mathrm{H} & -6.142182 & -4.017936 & -1.640188 \\ \mathrm{H} & -5.787017 & -5.654367 & 0.173526 \\ \mathrm{H} & -4.056645 & -5.233316 & 1.883233 \\ \mathrm{H} & -2.685926 & -3.191530 & 1.775943 \\ \mathrm{H} & -4.577238 & -0.000342 & -0.023019\end{array}$

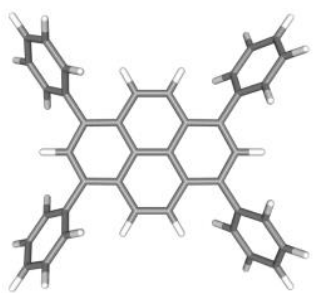

Charge

0

Multiplicity

1

Stoichiometry

$\mathrm{C} 40 \mathrm{H} 26$

Number of Basis Functions

1062 
Electronic Energy (Eh)

Mean of alpha and beta Electrons

Molecular Geometry in Cartesian Coordinates
$-1539.76456392$

133

$\begin{array}{lrrr}\mathrm{C} & 3.496191 & -0.000369 & 0.008071 \\ \mathrm{C} & 2.825852 & 1.213963 & -0.015901 \\ \mathrm{C} & 1.419787 & 1.229678 & -0.020923 \\ \mathrm{C} & 0.710900 & -0.000025 & -0.013194 \\ \mathrm{C} & 1.419507 & -1.229845 & -0.022799 \\ \mathrm{C} & 2.825621 & -1.214483 & -0.020047 \\ \mathrm{C} & 3.625637 & -2.453200 & -0.060007 \\ \mathrm{C} & 4.550950 & -2.717251 & 0.945939 \\ \mathrm{C} & 5.328463 & -3.860492 & 0.903580 \\ \mathrm{C} & 5.199677 & -4.750462 & -0.150003 \\ \mathrm{C} & 4.290358 & -4.490097 & -1.162255 \\ \mathrm{C} & 3.507472 & -3.350569 & -1.118606 \\ \mathrm{H} & 2.809233 & -3.141026 & -1.916987 \\ \mathrm{H} & 4.191726 & -5.177277 & -1.990519 \\ \mathrm{H} & 5.808461 & -5.642326 & -0.184270 \\ \mathrm{H} & 6.037903 & -4.057595 & 1.694675 \\ \mathrm{H} & 4.648056 & -2.024860 & 1.770341 \\ \mathrm{C} & 0.676268 & -2.444507 & -0.008489 \\ \mathrm{C} & -0.676740 & -2.444387 & -0.003538 \\ \mathrm{C} & -1.419776 & -1.229628 & 0.012942 \\ \mathrm{C} & -2.825920 & -1.214041 & 0.013784 \\ \mathrm{C} & -3.496300 & 0.000247 & -0.012454 \\ \mathrm{C} & -2.825730 & 1.214493 & 0.009825 \\ \mathrm{C} & -1.419591 & 1.229951 & 0.010965 \\ \mathrm{C} & -0.710982 & 0.000097 & 0.002337 \\ \mathrm{C} & -0.676287 & 2.444560 & -0.005298 \\ \mathrm{H} & -2.804953 & 3.148038 & 1.899764 \\ \mathrm{C} & 0.676727 & 2.444458 & -0.007399 \\ \mathrm{H} & 1.218131 & 3.378565 & 0.003822 \\ \mathrm{H} & -1.217440 & 3.378778 & -0.018524 \\ \mathrm{C} & -3.626029 & 2.453073 & 0.047506 \\ \mathrm{C} & -3.505371 & 3.354397 & 1.102468 \\ \mathrm{C} & -4.288612 & 4.493753 & 1.144104 \\ \mathrm{C} & -5.200971 & 4.749945 & 0.133529 \\ \mathrm{C} & -5.332439 & 3.855930 & -0.916294 \\ \mathrm{H} & -4.554431 & 2.712958 & -0.956699 \\ \mathrm{H} & -6.653646 & 2.017509 & -1.778276 \\ \mathrm{H} & -5.810089 & 5.641639 & -1.705958 \\ \mathrm{H} & -4.187893 & 5.184009 & 1.969561 \\ \mathrm{H} & -0.000362 & -0.004486\end{array}$




$\begin{array}{lrrr}\mathrm{C} & -3.626306 & -2.452256 & 0.058878 \\ \mathrm{C} & -4.559038 & -2.716020 & -0.940290 \\ \mathrm{C} & -5.337537 & -3.858344 & -0.891560 \\ \mathrm{C} & -5.202267 & -4.747771 & 0.161668 \\ \mathrm{C} & -4.285438 & -4.487754 & 1.167213 \\ \mathrm{C} & -3.501665 & -3.349113 & 1.117220 \\ \mathrm{H} & -2.797692 & -3.139764 & 1.910597 \\ \mathrm{H} & -4.181608 & -5.174486 & 1.995212 \\ \mathrm{H} & -5.811942 & -5.638820 & 0.200992 \\ \mathrm{H} & -6.053036 & -4.055041 & -1.677279 \\ \mathrm{H} & -4.661458 & -2.024125 & -1.764455 \\ \mathrm{H} & -1.218107 & -3.378511 & -0.015284 \\ \mathrm{H} & 1.217507 & -3.378718 & 0.001855 \\ \mathrm{C} & 3.626731 & 2.452455 & -0.048259 \\ \mathrm{C} & 3.515131 & 3.353443 & -1.104416 \\ \mathrm{C} & 4.299591 & 4.492214 & -1.140084 \\ \mathrm{C} & 5.203880 & 4.748147 & -0.122223 \\ \mathrm{C} & 5.325908 & 3.854652 & 0.929193 \\ \mathrm{C} & 4.546846 & 2.712220 & 0.963574 \\ \mathrm{H} & 4.638610 & 2.016961 & 1.786187 \\ \mathrm{H} & 6.031340 & 4.048289 & 1.724720 \\ \mathrm{H} & 5.813982 & 5.639327 & -0.150375 \\ \mathrm{H} & 4.206199 & 5.182228 & -1.966604 \\ \mathrm{H} & 2.820813 & 3.147492 & -1.907129 \\ \mathrm{H} & 4.577750 & -0.000413 & 0.003811\end{array}$

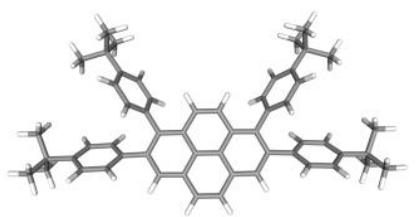

Charge

Multiplicity

Stoichiometry

Number of Basis Functions

Electronic Energy (Eh)

Mean of alpha and beta Electrons

0

1

C56H58

1638

$-2168.65163106$

197

Molecular Geometry in Cartesian Coordinates

$\begin{array}{lrrr}\mathrm{C} & 3.515429 & -1.774622 & 0.013778 \\ \mathrm{C} & 2.804929 & -2.966463 & 0.031857 \\ \mathrm{C} & 1.412000 & -2.987430 & 0.037208 \\ \mathrm{C} & 0.708107 & -1.758569 & -0.002310 \\ \mathrm{C} & -0.708680 & -1.758190 & -0.002015 \\ \mathrm{C} & -1.413106 & -2.986707 & 0.038545 \\ \mathrm{C} & -2.806017 & -2.965063 & 0.034629\end{array}$




\begin{tabular}{|c|c|c|c|}
\hline $\mathrm{C}$ & -3.515935 & -1.772889 & 0.015411 \\
\hline $\mathrm{C}$ & -2.832264 & -0.550818 & -0.017535 \\
\hline $\mathrm{C}$ & -1.427074 & -0.537111 & -0.046801 \\
\hline $\mathrm{C}$ & -0.676258 & 0.670046 & -0.171622 \\
\hline $\mathrm{C}$ & 0.676927 & 0.669728 & -0.171128 \\
\hline $\mathrm{C}$ & 1.427089 & -0.537841 & -0.046437 \\
\hline $\mathrm{C}$ & 2.832261 & -0.552201 & -0.016828 \\
\hline $\mathrm{C}$ & 3.598075 & 0.708183 & -0.038933 \\
\hline $\mathrm{C}$ & 4.510406 & 0.969991 & -1.056121 \\
\hline $\mathrm{C}$ & 5.250239 & 2.134712 & -1.055950 \\
\hline $\mathrm{C}$ & 5.117882 & 3.085411 & -0.045580 \\
\hline $\mathrm{C}$ & 4.198900 & 2.820605 & 0.962860 \\
\hline $\mathrm{C}$ & 3.450709 & 1.656568 & 0.966558 \\
\hline $\mathrm{H}$ & 2.755089 & 1.470029 & 1.773857 \\
\hline $\mathrm{H}$ & 4.052070 & 3.519285 & 1.770796 \\
\hline $\mathrm{C}$ & 5.972169 & 4.343702 & -0.081332 \\
\hline $\mathrm{C}$ & 5.692170 & 5.107085 & -1.384479 \\
\hline $\mathrm{H}$ & 5.944979 & 4.510311 & -2.256550 \\
\hline $\mathrm{H}$ & 4.638234 & 5.371994 & -1.446700 \\
\hline $\mathrm{H}$ & 6.280356 & 6.022042 & -1.416212 \\
\hline $\mathrm{C}$ & 5.687419 & 5.286054 & 1.092375 \\
\hline $\mathrm{H}$ & 5.903002 & 4.804328 & 2.043537 \\
\hline $\mathrm{H}$ & 6.321619 & 6.166828 & 1.011486 \\
\hline $\mathrm{H}$ & 4.651151 & 5.616884 & 1.086285 \\
\hline $\mathrm{C}$ & 7.452640 & 3.938757 & -0.022371 \\
\hline $\mathrm{H}$ & 8.085045 & 4.824127 & -0.039296 \\
\hline $\mathrm{H}$ & 7.724841 & 3.308338 & -0.864478 \\
\hline $\mathrm{H}$ & 7.653961 & 3.387378 & 0.894130 \\
\hline $\mathrm{H}$ & 5.948058 & 2.297157 & -1.863893 \\
\hline $\mathrm{H}$ & 4.634981 & 0.253371 & -1.855650 \\
\hline $\mathrm{H}$ & 1.216059 & 1.598760 & -0.284383 \\
\hline $\mathrm{H}$ & -1.214841 & 1.599324 & -0.285385 \\
\hline $\mathrm{C}$ & -3.597853 & 0.709658 & -0.043830 \\
\hline $\mathrm{C}$ & -4.508776 & 0.968184 & -1.063144 \\
\hline $\mathrm{C}$ & -5.248333 & 2.133056 & -1.068093 \\
\hline $\mathrm{C}$ & -5.117187 & 3.087205 & -0.060829 \\
\hline $\mathrm{C}$ & -4.199639 & 2.825669 & 0.949761 \\
\hline $\mathrm{C}$ & -3.451686 & 1.661474 & 0.958569 \\
\hline $\mathrm{H}$ & -2.757176 & 1.477774 & 1.767473 \\
\hline $\mathrm{H}$ & -4.053718 & 3.527132 & 1.755458 \\
\hline $\mathrm{C}$ & -5.971286 & 4.345452 & -0.102119 \\
\hline $\mathrm{C}$ & -5.688676 & 5.291407 & 1.069199 \\
\hline $\mathrm{H}$ & -5.905520 & 4.812438 & 2.021464 \\
\hline $\mathrm{H}$ & -6.323129 & 6.171660 & 0.984684 \\
\hline $\mathrm{H}$ & -4.652543 & 5.622676 & 1.063691 \\
\hline $\mathrm{C}$ & -7.451884 & 3.940723 & -0.044597 \\
\hline $\mathrm{H}$ & -7.654618 & 3.391084 & 0.872633 \\
\hline
\end{tabular}




\begin{tabular}{|c|c|c|c|}
\hline $\mathrm{H}$ & -7.722887 & 3.308700 & -0.885882 \\
\hline $\mathrm{H}$ & -8.084215 & 4.826087 & -0.064162 \\
\hline $\mathrm{C}$ & -5.688849 & 5.104822 & -1.407087 \\
\hline $\mathrm{H}$ & -5.939938 & 4.505340 & -2.277801 \\
\hline H & -6.277031 & 6.019643 & -1.442756 \\
\hline e & -4.634816 & 5.369617 & -1.468112 \\
\hline & -5.944931 & 2.292899 & -1.877611 \\
\hline $\mathrm{H}$ & -4.632378 & 0.248833 & -1.860379 \\
\hline$C$ & -4.990096 & -1.823724 & 0.043939 \\
\hline $\mathrm{C}$ & -5.726194 & -1.122604 & 0.993664 \\
\hline$C$ & -7.104626 & -1.183289 & 1.001157 \\
\hline $\mathrm{C}$ & -7.809845 & -1.940187 & 0.067441 \\
\hline $\mathrm{C}$ & -7.067136 & -2.651385 & -0.867169 \\
\hline $\mathrm{C}$ & -5.684372 & -2.599397 & -0.876125 \\
\hline $\mathrm{H}$ & -5.135071 & -3.154357 & -1.624621 \\
\hline $\mathrm{H}$ & -7.556490 & -3.259825 & -1.610688 \\
\hline $\mathrm{C}$ & -9.330582 & -1.953926 & 0.105679 \\
\hline $\mathrm{C}$ & -9.942386 & -2.834901 & -0.988036 \\
\hline $\mathrm{H}$ & -9.664709 & -2.480049 & -1.978084 \\
\hline $\mathrm{H}$ & -9.628022 & -3.870507 & -0.878542 \\
\hline $\mathrm{H}$ & -11.027702 & -2.803984 & -0.912741 \\
\hline $\mathrm{C}$ & -9.844495 & -0.519793 & -0.091153 \\
\hline $\mathrm{H}$ & -9.493819 & 0.138149 & 0.699132 \\
\hline $\mathrm{H}$ & -10.932564 & -0.507556 & -0.088124 \\
\hline $\mathrm{H}$ & -9.496477 & -0.123434 & -1.043197 \\
\hline $\mathrm{C}$ & -9.795917 & -2.490992 & 1.467410 \\
\hline $\mathrm{H}$ & -9.444296 & -1.864110 & 2.282292 \\
\hline $\mathrm{H}$ & -10.882957 & -2.521753 & 1.505934 \\
\hline $\mathrm{H}$ & -9.416014 & -3.498861 & 1.624282 \\
\hline $\mathrm{H}$ & -7.636119 & -0.622203 & 1.755358 \\
\hline $\mathrm{H}$ & -5.212681 & -0.531462 & 1.737945 \\
\hline $\mathrm{H}$ & -3.347891 & -3.900103 & 0.072200 \\
\hline $\mathrm{C}$ & -0.677141 & -4.207227 & 0.080515 \\
\hline $\mathrm{C}$ & 0.675469 & -4.207588 & 0.079641 \\
\hline $\mathrm{H}$ & 1.229876 & -5.135358 & 0.110263 \\
\hline $\mathrm{H}$ & -1.231994 & -5.134704 & 0.111923 \\
\hline $\mathrm{H}$ & 3.346363 & -3.901828 & 0.067716 \\
\hline $\mathrm{C}$ & 4.989628 & -1.825905 & 0.040519 \\
\hline $\mathrm{C}$ & 5.727165 & -1.126741 & 0.990561 \\
\hline $\mathrm{C}$ & 7.105648 & -1.186555 & 0.995183 \\
\hline $\mathrm{C}$ & 7.809505 & -1.940884 & 0.058371 \\
\hline $\mathrm{C}$ & 7.065374 & -2.650553 & -0.876277 \\
\hline $\mathrm{C}$ & 5.682561 & -2.599289 & -0.882486 \\
\hline $\mathrm{H}$ & 5.132150 & -3.152809 & -1.631232 \\
\hline $\mathrm{H}$ & 7.553609 & -3.257034 & -1.622128 \\
\hline $\mathrm{C}$ & 9.330363 & -1.952443 & 0.092511 \\
\hline $\mathrm{C}$ & 9.801017 & -2.485166 & 1.454097 \\
\hline
\end{tabular}




$\begin{array}{lccc}\mathrm{H} & 9.422646 & -3.492933 & 1.615215 \\ \mathrm{H} & 9.451932 & -1.856299 & 2.268514 \\ \mathrm{H} & 10.888223 & -2.514801 & 1.488770 \\ \mathrm{C} & 9.940382 & -2.835278 & -1.000699 \\ \mathrm{H} & 11.025811 & -2.804551 & -0.926975 \\ \mathrm{H} & 9.625807 & -3.870601 & -0.889098 \\ \mathrm{H} & 9.661501 & -2.481932 & -1.990942 \\ \mathrm{C} & 9.840945 & -0.517823 & -0.109504 \\ \mathrm{H} & 9.491275 & 0.141289 & 0.680274 \\ \mathrm{H} & 9.489184 & -0.124509 & -1.061439 \\ \mathrm{H} & 10.928992 & -0.503434 & -0.109870 \\ \mathrm{H} & 7.638265 & -0.626506 & 1.749356 \\ \mathrm{H} & 5.214783 & -0.537564 & 1.737178\end{array}$

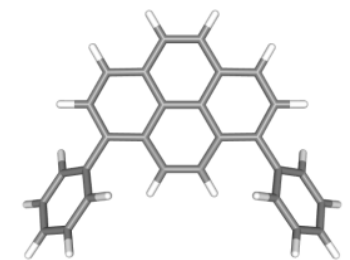

Charge

Multiplicity

Stoichiometry

Number of Basis Functions

Electronic Energy (Eh)

Mean of alpha and beta Electrons
0

1

C28H18

742

$-1077.72284428$

93

Molecular Geometry in Cartesian Coordinates

$\begin{array}{lrrr}\mathrm{C} & 3.496656 & 1.598901 & -0.036212 \\ \mathrm{C} & 2.829389 & 0.378963 & 0.023304 \\ \mathrm{C} & 1.423685 & 0.359513 & 0.055464 \\ \mathrm{C} & 0.709053 & 1.581649 & -0.024781 \\ \mathrm{C} & -0.709164 & 1.581703 & -0.025041 \\ \mathrm{C} & -1.423894 & 0.359603 & 0.054760 \\ \mathrm{C} & -2.829587 & 0.379167 & 0.022563 \\ \mathrm{C} & -3.496781 & 1.599130 & -0.036936 \\ \mathrm{C} & -2.808009 & 2.791725 & -0.099918 \\ \mathrm{C} & -1.412287 & 2.809617 & -0.106392 \\ \mathrm{C} & -0.676262 & 4.026424 & -0.189490 \\ \mathrm{C} & 0.676350 & 4.026370 & -0.189350 \\ \mathrm{C} & 1.412268 & 2.809518 & -0.106013 \\ \mathrm{C} & 2.807984 & 2.791546 & -0.099166 \\ \mathrm{H} & 3.347798 & 3.726210 & -0.154507 \\ \mathrm{H} & 1.230560 & 4.952419 & -0.251030 \\ \mathrm{H} & -1.230379 & 4.952516 & -0.251375\end{array}$




$\begin{array}{lrrr}\mathrm{H} & -3.347745 & 3.726422 & -0.155504 \\ \mathrm{H} & -4.577604 & 1.601096 & -0.056413 \\ \mathrm{C} & -3.625404 & -0.859600 & 0.031943 \\ \mathrm{C} & -3.413431 & -1.852660 & -0.921621 \\ \mathrm{C} & -4.187699 & -2.998136 & -0.925250 \\ \mathrm{C} & -5.182284 & -3.170071 & 0.023116 \\ \mathrm{C} & -5.404654 & -2.185616 & 0.971332 \\ \mathrm{C} & -4.636074 & -1.036229 & 0.972951 \\ \mathrm{H} & -4.804499 & -0.272056 & 1.718416 \\ \mathrm{H} & -6.180523 & -2.314143 & 1.712189 \\ \mathrm{H} & -5.784234 & -4.066845 & 0.020989 \\ \mathrm{H} & -4.016025 & -3.759363 & -1.672584 \\ \mathrm{H} & -2.649270 & -1.711929 & -1.672866 \\ \mathrm{C} & -0.676832 & -0.842962 & 0.223613 \\ \mathrm{C} & 0.676469 & -0.842967 & 0.224262 \\ \mathrm{H} & 1.216318 & -1.765747 & 0.377619 \\ \mathrm{H} & -1.216837 & -1.765757 & 0.376348 \\ \mathrm{C} & 3.625273 & -0.859749 & 0.032232 \\ \mathrm{C} & 3.412331 & -1.853157 & -0.920762 \\ \mathrm{C} & 4.186840 & -2.998464 & -0.924989 \\ \mathrm{C} & 5.182716 & -3.169861 & 0.022116 \\ \mathrm{C} & 5.406063 & -2.185064 & 0.969748 \\ \mathrm{C} & 4.637151 & -1.035908 & 0.972048 \\ \mathrm{H} & 4.806325 & -0.271503 & 1.717106 \\ \mathrm{H} & 6.182927 & -2.313153 & 1.709638 \\ \mathrm{H} & 5.784892 & -4.066480 & 0.019467 \\ \mathrm{H} & 4.014366 & -3.759987 & -1.671836 \\ \mathrm{H} & 2.647263 & -1.712802 & -1.671152 \\ \mathrm{H} & 4.577478 & 1.600798 & -0.055685\end{array}$

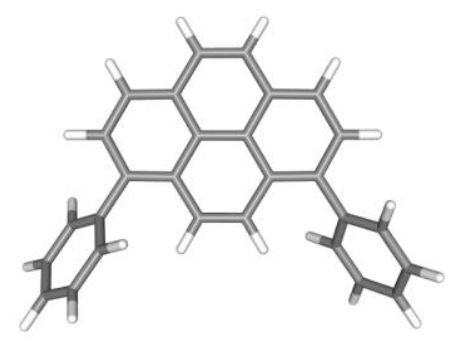

Charge

0

Multiplicity

Stoichiometry

Number of Basis Functions

Electronic Energy (Eh)

Mean of alpha and beta Electrons

1

C28H18

742

$-1077.72249468$

93

Molecular Geometry in Cartesian Coordinates 


\begin{tabular}{|c|c|c|c|}
\hline $\mathrm{C}$ & 3.495535 & 1.594167 & 0.087795 \\
\hline $\mathrm{C}$ & 2.830317 & 0.373928 & 0.018786 \\
\hline $\mathrm{C}$ & 1.424464 & 0.350825 & 0.003934 \\
\hline $\mathrm{C}$ & 0.709026 & 1.575131 & 0.012488 \\
\hline $\mathrm{C}$ & -0.709033 & 1.575038 & -0.013740 \\
\hline $\mathrm{C}$ & -1.424275 & 0.350617 & -0.006488 \\
\hline $\mathrm{C}$ & -2.830143 & 0.373502 & -0.020201 \\
\hline $\mathrm{C}$ & -3.495589 & 1.593711 & -0.087688 \\
\hline $\mathrm{C}$ & -2.806148 & 2.787682 & -0.096386 \\
\hline $\mathrm{C}$ & -1.411524 & 2.805771 & -0.044420 \\
\hline $\mathrm{C}$ & -0.676016 & 4.025608 & -0.023759 \\
\hline $\mathrm{C}$ & 0.675579 & 4.025683 & 0.026067 \\
\hline $\mathrm{C}$ & 1.411298 & 2.805942 & 0.044969 \\
\hline $\mathrm{C}$ & 2.805911 & 2.788029 & 0.097271 \\
\hline $\mathrm{H}$ & 3.344505 & 3.724205 & 0.136451 \\
\hline $\mathrm{H}$ & 1.229376 & 4.953762 & 0.048274 \\
\hline $\mathrm{H}$ & -1.229972 & 4.953622 & -0.044645 \\
\hline $\mathrm{H}$ & -3.344913 & 3.723808 & -0.134386 \\
\hline $\mathrm{H}$ & -4.576436 & 1.596681 & -0.107435 \\
\hline $\mathrm{C}$ & -3.632190 & -0.860205 & 0.050928 \\
\hline $\mathrm{C}$ & -3.485993 & -1.748014 & 1.114069 \\
\hline $\mathrm{C}$ & -4.268368 & -2.885559 & 1.189868 \\
\hline $\mathrm{C}$ & -5.205602 & -3.153697 & 0.206068 \\
\hline $\mathrm{C}$ & -5.362915 & -2.273141 & -0.851024 \\
\hline $\mathrm{C}$ & -4.586077 & -1.131639 & -0.925746 \\
\hline $\mathrm{H}$ & -4.704064 & -0.447924 & -1.754438 \\
\hline $\mathrm{H}$ & -6.094527 & -2.476436 & -1.619649 \\
\hline $\mathrm{H}$ & -5.813968 & -4.044177 & 0.265227 \\
\hline $\mathrm{H}$ & -4.148026 & -3.564808 & 2.021480 \\
\hline $\mathrm{H}$ & -2.767344 & -1.530900 & 1.891647 \\
\hline $\mathrm{C}$ & -0.676471 & -0.863134 & -0.007332 \\
\hline $\mathrm{C}$ & 0.676837 & -0.863039 & 0.002466 \\
\hline $\mathrm{H}$ & 1.215813 & -1.798679 & 0.018776 \\
\hline $\mathrm{H}$ & -1.215327 & -1.798813 & -0.025486 \\
\hline $\mathrm{C}$ & 3.632527 & -0.859738 & -0.051614 \\
\hline $\mathrm{C}$ & 3.488534 & -1.747125 & -1.115403 \\
\hline $\mathrm{C}$ & 4.270586 & -2.884990 & -1.189641 \\
\hline $\mathrm{C}$ & 5.205260 & -3.153890 & -0.203608 \\
\hline $\mathrm{C}$ & 5.360540 & -2.273638 & 0.854023 \\
\hline $\mathrm{C}$ & 4.584061 & -1.131780 & 0.927168 \\
\hline $\mathrm{H}$ & 4.700449 & -0.448285 & 1.756268 \\
\hline $\mathrm{H}$ & 6.090244 & -2.477422 & 1.624329 \\
\hline $\mathrm{H}$ & 5.813188 & -4.044754 & -0.261457 \\
\hline $\mathrm{H}$ & 4.151964 & -3.563965 & -2.021721 \\
\hline $\mathrm{H}$ & 2.771873 & -1.529440 & -1.894654 \\
\hline $\mathrm{H}$ & 4.576369 & 1.597264 & 0.108322 \\
\hline
\end{tabular}




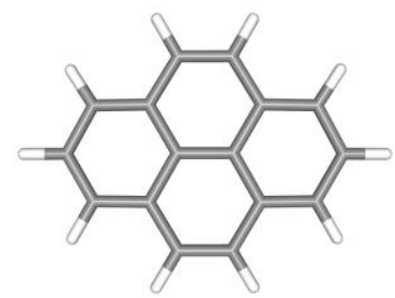

Charge

Multiplicity

Stoichiometry

Number of Basis Functions

Electronic Energy (Eh)

Mean of alpha and beta Electrons
0

1

$\mathrm{C} 16 \mathrm{H} 10$

422

$-615.678614151$

53

Molecular Geometry in Cartesian Coordinates

$\begin{array}{lrrr}\mathrm{C} & -3.496639 & -0.011839 & 0.013111 \\ \mathrm{C} & -2.830015 & 1.208394 & -0.040787 \\ \mathrm{C} & -1.424165 & 1.231397 & -0.025449 \\ \mathrm{C} & -0.709154 & 0.007030 & -0.002364 \\ \mathrm{C} & 0.709141 & 0.007029 & 0.001846 \\ \mathrm{C} & 1.424141 & 1.231389 & 0.025268 \\ \mathrm{C} & 2.829978 & 1.208402 & 0.041714 \\ \mathrm{C} & 3.496642 & -0.011813 & -0.012294 \\ \mathrm{C} & 2.807469 & -1.205724 & -0.036587 \\ \mathrm{C} & 1.412050 & -1.223739 & -0.014575 \\ \mathrm{C} & 0.676190 & -2.443531 & -0.010522 \\ \mathrm{C} & -0.676163 & -2.443534 & 0.010286 \\ \mathrm{C} & -1.412040 & -1.223748 & 0.014212 \\ \mathrm{C} & -2.807453 & -1.205761 & 0.036585 \\ \mathrm{H} & -3.346824 & -2.141921 & 0.063569 \\ \mathrm{H} & -1.230369 & -3.371584 & 0.019981 \\ \mathrm{H} & 1.230407 & -3.371574 & -0.020137 \\ \mathrm{H} & 3.346866 & -2.141867 & -0.063644 \\ \mathrm{H} & 4.577663 & -0.014856 & -0.008843 \\ \mathrm{C} & 0.676605 & 2.445198 & 0.009189 \\ \mathrm{C} & -0.676601 & 2.445203 & -0.010058 \\ \mathrm{H} & -1.215742 & 3.380874 & -0.004691 \\ \mathrm{H} & 1.215788 & 3.380846 & 0.003232 \\ \mathrm{H} & -4.577664 & -0.014865 & 0.010436 \\ \mathrm{H} & -3.411364 & 2.104475 & -0.103809 \\ \mathrm{H} & 3.411320 & 2.104364 & 0.106466\end{array}$




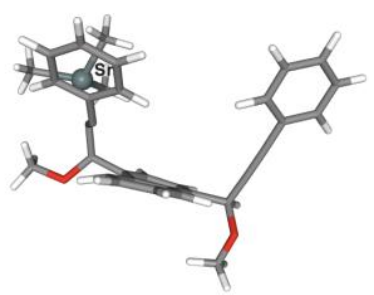

Charge

Multiplicity

Stoichiometry

Number of Basis Functions

Electronic Energy (Eh)

Number of Imaginary Frequencies

Mean of alpha and beta Electrons
0

2

C33H33O2Sn

389

$-1430.25910786$

1

125.5

Molecular Geometry in Cartesian Coordinates

$\begin{array}{llll}\mathrm{C} & 1.421380 & -3.005143 & -1.322549 \\ \mathrm{C} & 0.006280 & -2.922927 & -1.254492 \\ \mathrm{C} & 2.213367 & -2.480510 & -0.315869 \\ \mathrm{C} & -0.608464 & -2.373476 & -0.141780 \\ \mathrm{C} & 0.180868 & -1.913969 & 0.953378 \\ \mathrm{C} & 1.604008 & -1.904720 & 0.849558 \\ \mathrm{C} & 2.373398 & -1.302519 & 1.899135 \\ \mathrm{H} & 3.455274 & -1.324086 & 1.841426 \\ \mathrm{C} & 1.737647 & -0.723100 & 2.990907 \\ \mathrm{C} & 0.330599 & -0.765527 & 3.123391 \\ \mathrm{C} & -0.463597 & -1.344633 & 2.116994 \\ \mathrm{H} & 2.332670 & -0.270966 & 3.778417 \\ \mathrm{H} & -0.140344 & -0.372552 & 4.018720 \\ \mathrm{H} & -1.468725 & -1.678326 & 2.353384 \\ \mathrm{C} & -2.085829 & -2.064434 & -0.159586 \\ \mathrm{H} & -2.506188 & -2.395641 & -1.122768 \\ \mathrm{C} & -4.190041 & -2.630632 & 0.900857 \\ \mathrm{H} & -4.614544 & -2.863191 & -0.088149 \\ \mathrm{H} & -4.588267 & -3.326804 & 1.639263 \\ \mathrm{H} & -4.461323 & -1.602006 & 1.173842 \\ \mathrm{O} & -2.755169 & -2.810602 & 0.896826 \\ \mathrm{C} & -2.294478 & -0.536393 & -0.004126 \\ \mathrm{C} & -1.730845 & 0.120653 & 1.003027 \\ \mathrm{C} & -1.732406 & 1.428575 & 1.606408 \\ \mathrm{C} & -2.938154 & 1.988392 & 2.098005 \\ \mathrm{C} & -0.527984 & 2.156631 & 1.755234 \\ \mathrm{C} & -2.941218 & 3.256245 & 2.690129 \\ \mathrm{H} & -3.859215 & 1.416822 & 2.013286 \\ \mathrm{C} & -0.540529 & 3.426687 & 2.341367\end{array}$




$\begin{array}{lrrr}\mathrm{H} & 0.399673 & 1.715095 & 1.401267 \\ \mathrm{C} & -1.744294 & 3.983364 & 2.809074 \\ \mathrm{H} & -3.871203 & 3.677863 & 3.058273 \\ \mathrm{H} & 0.386222 & 3.982956 & 2.438705 \\ \mathrm{H} & -1.747948 & 4.967170 & 3.266758 \\ \mathrm{Sn} & -3.372886 & 0.557931 & -1.504147 \\ \mathrm{C} & -3.068951 & -0.489961 & -3.337392 \\ \mathrm{H} & -3.390955 & 0.118749 & -4.190026 \\ \mathrm{H} & -2.007033 & -0.727659 & -3.469517 \\ \mathrm{H} & -3.638700 & -1.426346 & -3.355639 \\ \mathrm{C} & -5.452504 & 0.568543 & -1.023712 \\ \mathrm{H} & -5.617331 & 1.036397 & -0.046342 \\ \mathrm{H} & -6.018607 & 1.133413 & -1.773158 \\ \mathrm{H} & -5.852284 & -0.451312 & -0.990729 \\ \mathrm{C} & -2.594340 & 2.531758 & -1.575111 \\ \mathrm{H} & -2.915906 & 3.105636 & -0.698861 \\ \mathrm{H} & -1.499130 & 2.501244 & -1.572161 \\ \mathrm{H} & -2.926128 & 3.055786 & -2.478617 \\ \mathrm{H} & -0.594573 & -3.268570 & -2.091098 \\ \mathrm{H} & 1.888905 & -3.446983 & -2.198711 \\ \mathrm{C} & 3.727918 & -2.495035 & -0.492362 \\ \mathrm{H} & 3.951483 & -3.059804 & -1.411680 \\ \mathrm{C} & 4.272620 & -1.134697 & -0.638097 \\ \mathrm{C} & 4.695155 & -0.001040 & -0.776734 \\ \mathrm{C} & 5.161920 & 1.353803 & -0.910044 \\ \mathrm{C} & 6.271960 & 1.657722 & -1.724345 \\ \mathrm{C} & 4.504314 & 2.395172 & -0.221723 \\ \mathrm{C} & 6.714440 & 2.980378 & -1.848604 \\ \mathrm{H} & 6.778114 & 0.854388 & -2.249583 \\ \mathrm{C} & 4.950355 & 3.716084 & -0.348507 \\ \mathrm{H} & 3.650785 & 2.154530 & 0.404680 \\ \mathrm{C} & 6.055710 & 4.013115 & -1.162105 \\ \mathrm{H} & 7.569802 & 3.207214 & -2.476534 \\ \mathrm{H} & 4.439386 & 4.512318 & 0.182986 \\ \mathrm{H} & 6.400540 & 5.037276 & -1.259520 \\ \mathrm{H} & 4.017006 & -4.502846 & 0.836517 \\ \mathrm{H} & 2.979344 & -4.556585 & 1.186827 \\ \mathrm{H} & 4.117080 & -5.092511 & -0.086161 \\ \mathrm{H} & 4.426981 & -3.129979 & 0.616718\end{array}$

Frequencies (Top 10 out of 201)

1. $-406.3948 \mathrm{~cm}-1$ (Symmetry: A) *

2. $\quad 8.9525 \mathrm{~cm}-1$ (Symmetry: A)

3. $\quad 13.2039 \mathrm{~cm}-1$ (Symmetry: A)

4. $21.6131 \mathrm{~cm}-1$ (Symmetry: A) 
5. $\quad 25.7473 \mathrm{~cm}-1$ (Symmetry: A)

6. $\quad 31.9521 \mathrm{~cm}-1$ (Symmetry: A)

7. $\quad 34.9802 \mathrm{~cm}-1$ (Symmetry: A)

8. $44.1861 \mathrm{~cm}-1$ (Symmetry: A)

9. $\quad 54.4316 \mathrm{~cm}-1$ (Symmetry: A)

10. $\quad 61.1379 \mathrm{~cm}-1$ (Symmetry: A)

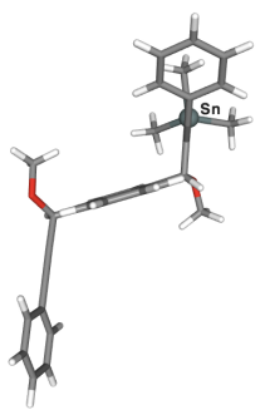

Charge

Multiplicity

Stoichiometry

Number of Basis Functions

0

Electronic Energy (Eh)

Number of Imaginary Frequencies

Mean of alpha and beta Electrons

2

C33H33O2Sn
389
-1430.28346831
1
125.5

Molecular Geometry in Cartesian Coordinates

$\begin{array}{lrrr}\mathrm{C} & 2.163054 & 2.846895 & -0.572204 \\ \mathrm{C} & 2.535706 & 1.807883 & 0.259473 \\ \mathrm{C} & 1.790978 & 0.587774 & 0.281434 \\ \mathrm{C} & 0.644571 & 0.454216 & -0.569458 \\ \mathrm{C} & 0.288511 & 1.548915 & -1.417387 \\ \mathrm{C} & 1.029344 & 2.716222 & -1.420222 \\ \mathrm{C} & 2.157676 & -0.502829 & 1.142620 \\ \mathrm{C} & -0.118616 & -0.764417 & -0.537165 \\ \mathrm{C} & 0.250270 & -1.782103 & 0.321141 \\ \mathrm{C} & 1.390076 & -1.649462 & 1.159021 \\ \mathrm{H} & 2.734087 & 3.769382 & -0.577385 \\ \mathrm{H} & 3.386950 & 1.915092 & 0.921454 \\ \mathrm{H} & -0.583227 & 1.477021 & -2.058956 \\ \mathrm{H} & 0.740887 & 3.539372 & -2.065392 \\ \mathrm{C} & -1.374337 & -0.918433 & -1.384619 \\ \mathrm{H} & -1.212892 & -0.519198 & -2.396083 \\ \mathrm{C} & -0.976439 & -3.104725 & -2.408947 \\ \mathrm{H} & 0.080454 & -3.104376 & -2.116603 \\ \mathrm{H} & -1.371284 & -4.120312 & -2.363674\end{array}$




\begin{tabular}{|c|c|c|c|}
\hline $\mathrm{H}$ & -1.073764 & -2.716437 & -3.430285 \\
\hline $\mathrm{O}$ & -1.770931 & -2.313083 & -1.492149 \\
\hline $\mathrm{C}$ & -2.572850 & -0.238158 & -0.719367 \\
\hline $\mathrm{C}$ & -2.830865 & 1.043014 & -0.727451 \\
\hline $\mathrm{C}$ & -3.408535 & 2.299471 & -0.522268 \\
\hline $\mathrm{C}$ & -3.003494 & 3.122235 & 0.575786 \\
\hline $\mathrm{C}$ & -4.401625 & 2.808444 & -1.417972 \\
\hline $\mathrm{C}$ & -3.580835 & 4.376457 & 0.769253 \\
\hline $\mathrm{H}$ & -2.238046 & 2.751813 & 1.250547 \\
\hline $\mathrm{C}$ & -4.967139 & 4.065326 & -1.207646 \\
\hline $\mathrm{H}$ & -4.710417 & 2.196166 & -2.259692 \\
\hline $\mathrm{C}$ & -4.566163 & 4.859718 & -0.115069 \\
\hline $\mathrm{H}$ & -3.266750 & 4.986001 & 1.610688 \\
\hline $\mathrm{H}$ & -5.724648 & 4.433292 & -1.892554 \\
\hline $\mathrm{H}$ & -5.010662 & 5.836665 & 0.042255 \\
\hline $\mathrm{Sn}$ & -3.933061 & -1.618333 & 0.251221 \\
\hline $\mathrm{C}$ & -5.059822 & -2.709149 & -1.183945 \\
\hline $\mathrm{H}$ & -4.407415 & -3.434503 & -1.679964 \\
\hline $\mathrm{H}$ & -5.893115 & -3.240410 & -0.710008 \\
\hline $\mathrm{H}$ & -5.468285 & -2.033113 & -1.942909 \\
\hline $\mathrm{C}$ & -2.931405 & -2.916776 & 1.610160 \\
\hline $\mathrm{H}$ & -2.457294 & -3.735694 & 1.058614 \\
\hline $\mathrm{H}$ & -2.158393 & -2.372998 & 2.164204 \\
\hline $\mathrm{H}$ & -3.642608 & -3.338839 & 2.329745 \\
\hline $\mathrm{C}$ & -5.216436 & -0.303419 & 1.334273 \\
\hline $\mathrm{H}$ & -6.017399 & -0.853483 & 1.842252 \\
\hline $\mathrm{H}$ & -4.646367 & 0.251253 & 2.088998 \\
\hline $\mathrm{H}$ & -5.675814 & 0.429710 & 0.660138 \\
\hline $\mathrm{H}$ & 1.660331 & -2.469826 & 1.819147 \\
\hline $\mathrm{H}$ & -0.342659 & -2.690449 & 0.349337 \\
\hline $\mathrm{C}$ & 3.385215 & -0.427509 & 2.046034 \\
\hline $\mathrm{H}$ & 3.406455 & -1.350162 & 2.647857 \\
\hline $\mathrm{C}$ & 2.190989 & 0.726866 & 3.814243 \\
\hline $\mathrm{H}$ & 1.272989 & 0.897042 & 3.238608 \\
\hline $\mathrm{H}$ & 2.100485 & -0.219968 & 4.365599 \\
\hline $\mathrm{H}$ & 2.345951 & 1.546304 & 4.515767 \\
\hline $\mathrm{O}$ & 3.355916 & 0.712119 & 2.952425 \\
\hline $\mathrm{C}$ & 4.639779 & -0.347448 & 1.278311 \\
\hline $\mathrm{C}$ & 5.671008 & -0.311024 & 0.631434 \\
\hline $\mathrm{C}$ & 6.887383 & -0.252589 & -0.136458 \\
\hline $\mathrm{C}$ & 7.551751 & 0.977191 & -0.323578 \\
\hline $\mathrm{C}$ & 7.427438 & -1.422389 & -0.709210 \\
\hline $\mathrm{C}$ & 8.735109 & 1.032803 & -1.069943 \\
\hline $\mathrm{H}$ & 7.134979 & 1.876369 & 0.117913 \\
\hline $\mathrm{C}$ & 8.610161 & -1.361503 & -1.456058 \\
\hline $\mathrm{H}$ & 6.915721 & -2.368140 & -0.564580 \\
\hline $\mathrm{C}$ & 9.268182 & -0.134853 & -1.638919 \\
\hline
\end{tabular}




$\begin{array}{cccc}\mathrm{H} & 9.239791 & 1.983210 & -1.208879 \\ \mathrm{H} & 9.018492 & -2.266637 & -1.893418 \\ \mathrm{H} & 10.184779 & -0.089632 & -2.217803\end{array}$

Frequencies (Top 10 out of 201)

1. $\quad-3.4715 \mathrm{~cm}-1$ (Symmetry: A) *

2. $\quad 14.4302 \mathrm{~cm}-1$ (Symmetry: A)

3. $\quad 18.2290 \mathrm{~cm}-1$ (Symmetry: A)

4. $\quad 19.9648 \mathrm{~cm}-1$ (Symmetry: A)

5. $\quad 23.8671 \mathrm{~cm}-1$ (Symmetry: A)

6. $29.5298 \mathrm{~cm}-1$ (Symmetry: A)

7. $\quad 38.4936 \mathrm{~cm}-1$ (Symmetry: A)

8. $\quad 45.5036 \mathrm{~cm}-1$ (Symmetry: A)

9. $\quad 48.0333 \mathrm{~cm}-1$ (Symmetry: A)

10. $\quad 62.4296 \mathrm{~cm}-1$ (Symmetry: A)

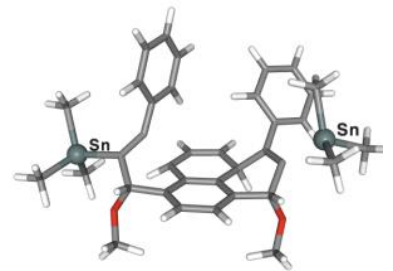

Charge

0

Multiplicity

3

Stoichiometry

Number of Basis Functions

$\mathrm{C} 36 \mathrm{H} 42 \mathrm{O} 2 \mathrm{Sn} 2$

442

Electronic Energy (Eh)

$-1553.29088577$

Number of Imaginary Frequencies

Mean of alpha and beta Electrons

1

141

Molecular Geometry in Cartesian Coordinates

$\begin{array}{lrrr}\mathrm{C} & 0.935082 & -2.873616 & -1.250793 \\ \mathrm{C} & -0.457913 & -3.012814 & -1.118926 \\ \mathrm{C} & 1.639750 & -1.988345 & -0.432574 \\ \mathrm{C} & -1.141357 & -2.250990 & -0.167696 \\ \mathrm{C} & -0.438740 & -1.373284 & 0.676600 \\ \mathrm{C} & 0.958372 & -1.243847 & 0.568060 \\ \mathrm{C} & 1.687359 & -0.273134 & 1.413590 \\ \mathrm{H} & 2.672073 & -0.568553 & 1.762873 \\ \mathrm{C} & 0.899307 & 0.543179 & 2.324164 \\ \mathrm{C} & -0.455952 & 0.467268 & 2.406874 \\ \mathrm{C} & -1.260468 & -0.565731 & 1.663522 \\ \mathrm{H} & 1.437027 & 1.225122 & 2.978308\end{array}$




\begin{tabular}{|c|c|c|c|}
\hline $\mathrm{H}$ & -0.997130 & 1.101350 & 3.104669 \\
\hline $\mathrm{H}$ & -1.638951 & -1.278650 & 2.419237 \\
\hline $\mathrm{C}$ & -2.651730 & -2.299138 & -0.027349 \\
\hline $\mathrm{H}$ & -3.077331 & -2.803840 & -0.909225 \\
\hline $\mathrm{C}$ & -2.666009 & -4.449924 & 1.123302 \\
\hline $\mathrm{H}$ & -1.577078 & -4.558985 & 1.199821 \\
\hline $\mathrm{H}$ & -3.145959 & -4.929829 & 1.976889 \\
\hline $\mathrm{H}$ & -3.008599 & -4.926257 & 0.192741 \\
\hline $\mathrm{O}$ & -3.060400 & -3.056996 & 1.161855 \\
\hline $\mathrm{C}$ & -3.225585 & -0.898146 & 0.150284 \\
\hline $\mathrm{C}$ & -2.550204 & -0.064604 & 0.976252 \\
\hline $\mathrm{C}$ & -3.032816 & 1.316860 & 1.262414 \\
\hline $\mathrm{C}$ & -2.301709 & 2.430990 & 0.804993 \\
\hline $\mathrm{C}$ & -4.245824 & 1.524062 & 1.943787 \\
\hline $\mathrm{C}$ & -2.794704 & 3.727590 & 0.999385 \\
\hline $\mathrm{H}$ & -1.365610 & 2.277611 & 0.272860 \\
\hline $\mathrm{C}$ & -4.735261 & 2.824620 & 2.147791 \\
\hline $\mathrm{H}$ & -4.797140 & 0.664007 & 2.315924 \\
\hline $\mathrm{C}$ & -4.014248 & 3.928725 & 1.669397 \\
\hline $\mathrm{H}$ & -2.237099 & 4.577590 & 0.618655 \\
\hline $\mathrm{H}$ & -5.673412 & 2.972758 & 2.673212 \\
\hline $\mathrm{H}$ & -4.395956 & 4.933919 & 1.816632 \\
\hline $\mathrm{Sn}$ & -5.009889 & -0.355636 & -0.888954 \\
\hline $\mathrm{C}$ & -5.210879 & -1.799388 & -2.449182 \\
\hline $\mathrm{H}$ & -5.394105 & -2.797681 & -2.033764 \\
\hline $\mathrm{H}$ & -6.056573 & -1.543229 & -3.097928 \\
\hline $\mathrm{H}$ & -4.308377 & -1.841679 & -3.070434 \\
\hline $\mathrm{C}$ & -6.722233 & -0.487911 & 0.377064 \\
\hline $\mathrm{H}$ & -6.560110 & -1.240487 & 1.156867 \\
\hline $\mathrm{H}$ & -6.916603 & 0.478224 & 0.856446 \\
\hline $\mathrm{H}$ & -7.609275 & -0.772845 & -0.200275 \\
\hline $\mathrm{C}$ & -4.828882 & 1.606563 & -1.689300 \\
\hline $\mathrm{H}$ & -5.630421 & 1.812856 & -2.408015 \\
\hline $\mathrm{H}$ & -4.881552 & 2.347588 & -0.882097 \\
\hline $\mathrm{H}$ & -3.865323 & 1.727340 & -2.196648 \\
\hline $\mathrm{H}$ & -1.008018 & -3.686626 & -1.770974 \\
\hline $\mathrm{H}$ & 1.467114 & -3.430089 & -2.018791 \\
\hline $\mathrm{C}$ & 3.115979 & -1.733779 & -0.676070 \\
\hline $\mathrm{H}$ & 3.390772 & -2.136656 & -1.665923 \\
\hline $\mathrm{C}$ & 3.907979 & -3.836925 & 0.302195 \\
\hline $\mathrm{H}$ & 2.914528 & -4.187276 & 0.605147 \\
\hline $\mathrm{H}$ & 4.136972 & -4.221108 & -0.702497 \\
\hline $\mathrm{H}$ & 4.656848 & -4.192707 & 1.010798 \\
\hline $\mathrm{C}$ & 3.475787 & -0.263717 & -0.567718 \\
\hline $\mathrm{C}$ & 2.522717 & 0.677279 & -0.317185 \\
\hline $\mathrm{C}$ & 2.061395 & 1.945087 & -0.720544 \\
\hline $\mathrm{C}$ & 2.348620 & 2.358844 & -2.068363 \\
\hline
\end{tabular}




$\begin{array}{lrrr}\mathrm{C} & 1.346831 & 2.862826 & 0.110154 \\ \mathrm{C} & 1.937237 & 3.601220 & -2.540374 \\ \mathrm{H} & 2.900335 & 1.675105 & -2.706796 \\ \mathrm{C} & 0.944297 & 4.104610 & -0.380519 \\ \mathrm{H} & 1.127439 & 2.592796 & 1.134430 \\ \mathrm{C} & 1.228088 & 4.489694 & -1.704827 \\ \mathrm{H} & 2.169667 & 3.889114 & -3.561105 \\ \mathrm{H} & 0.416880 & 4.788486 & 0.278598 \\ \mathrm{H} & 0.912435 & 5.458968 & -2.075493 \\ \mathrm{O} & 3.975744 & -2.391079 & 0.320835 \\ \mathrm{Sn} & 5.504901 & 0.118847 & 0.025465 \\ \mathrm{C} & 5.712071 & -0.124269 & 2.130756 \\ \mathrm{H} & 5.445198 & -1.151182 & 2.399823 \\ \mathrm{H} & 6.739090 & 0.077272 & 2.456253 \\ \mathrm{H} & 5.044475 & 0.564166 & 2.662482 \\ \mathrm{C} & 5.852750 & 2.157876 & -0.490989 \\ \mathrm{H} & 6.803697 & 2.516490 & -0.079852 \\ \mathrm{H} & 5.877201 & 2.284758 & -1.579369 \\ \mathrm{H} & 5.046842 & 2.790651 & -0.099614 \\ \mathrm{C} & 6.799123 & -1.169776 & -1.069269 \\ \mathrm{H} & 6.581441 & -2.210262 & -0.806058 \\ \mathrm{H} & 6.651493 & -1.040960 & -2.147492 \\ \mathrm{H} & 7.850382 & -0.960955 & -0.840812\end{array}$

Frequencies (Top 10 out of 240)

1. $-798.7815 \mathrm{~cm}-1$ (Symmetry: A) *

2. $\quad 8.2598 \mathrm{~cm}-1$ (Symmetry: A)

3. $\quad 16.3156 \mathrm{~cm}-1$ (Symmetry: A)

4. $\quad 23.7639 \mathrm{~cm}-1$ (Symmetry: A)

5. $26.3841 \mathrm{~cm}-1$ (Symmetry: A)

6. $\quad 31.1928 \mathrm{~cm}-1$ (Symmetry: A)

7. $\quad 37.7729 \mathrm{~cm}-1$ (Symmetry: A)

8. $\quad 42.9065 \mathrm{~cm}-1$ (Symmetry: A)

9. $\quad 48.5079 \mathrm{~cm}-1$ (Symmetry: A)

10. $\quad 59.0906 \mathrm{~cm}-1$ (Symmetry: A)

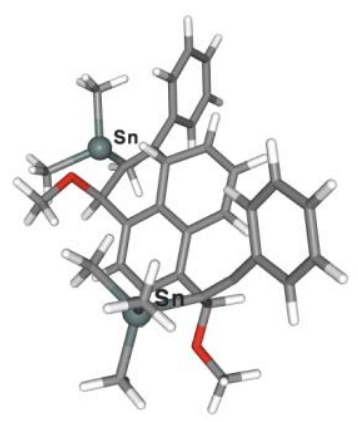


Charge 0

Multiplicity 3

Stoichiometry

Number of Basis Functions

Electronic Energy (Eh)

Number of Imaginary Frequencies

Mean of alpha and beta Electrons

\author{
$\mathrm{C} 36 \mathrm{H} 42 \mathrm{O} 2 \mathrm{Sn} 2$ \\ 442 \\ $-1553.33123428$ \\ 0 \\ 141
}

Molecular Geometry in Cartesian Coordinates

$\begin{array}{lrrr}\mathrm{C} & -1.057249 & -2.013317 & 1.400034 \\ \mathrm{C} & 0.201840 & -2.281397 & 0.839210 \\ \mathrm{C} & -1.551250 & -0.707728 & 1.432434 \\ \mathrm{C} & 0.963065 & -1.244442 & 0.286273 \\ \mathrm{C} & 0.486305 & 0.073947 & 0.315326 \\ \mathrm{C} & -0.771546 & 0.367020 & 0.909484 \\ \mathrm{C} & -1.193856 & 1.745330 & 1.019394 \\ \mathrm{H} & -2.153420 & 1.988425 & 1.462537 \\ \mathrm{C} & -0.318896 & 2.793851 & 0.648634 \\ \mathrm{C} & 0.911129 & 2.553625 & 0.077146 \\ \mathrm{C} & 1.357746 & 1.163695 & -0.296632 \\ \mathrm{H} & -0.624881 & 3.818356 & 0.839303 \\ \mathrm{H} & 1.566598 & 3.382455 & -0.169214 \\ \mathrm{H} & 1.242461 & 1.066529 & -1.396513 \\ \mathrm{C} & 2.316483 & -1.510993 & -0.331613 \\ \mathrm{H} & 2.684811 & -2.488418 & 0.021684 \\ \mathrm{C} & 1.472142 & -2.693595 & -2.294691 \\ \mathrm{H} & 0.407887 & -2.585188 & -2.051426 \\ \mathrm{H} & 1.602497 & -2.702204 & -3.377607 \\ \mathrm{H} & 1.845146 & -3.638466 & -1.871393 \\ \mathrm{O} & 2.243970 & -1.572054 & -1.798923 \\ \mathrm{C} & 3.313713 & -0.402022 & -0.041650 \\ \mathrm{C} & 2.855205 & 0.870524 & -0.050682 \\ \mathrm{C} & 3.800040 & 2.015215 & 0.105017 \\ \mathrm{C} & 4.012373 & 2.933717 & -0.941911 \\ \mathrm{C} & 4.540149 & 2.154551 & 1.293299 \\ \mathrm{C} & 4.960907 & 3.956291 & -0.810084 \\ \mathrm{H} & 3.456814 & 2.822837 & -1.869889 \\ \mathrm{C} & 5.490035 & 3.179202 & 1.428339 \\ \mathrm{H} & 4.359591 & 1.460446 & 2.109664 \\ \mathrm{C} & 5.705869 & 4.080477 & 0.375318 \\ \mathrm{H} & 5.127643 & 4.647509 & -1.629924 \\ \mathrm{H} & 6.054546 & 3.273229 & 2.350655 \\ \mathrm{H} & 6.442420 & 4.870993 & 0.475782 \\ \mathrm{Sn} & 5.380781 & -0.942706 & -0.067242\end{array}$




\begin{tabular}{|c|c|c|c|}
\hline $\mathrm{C}$ & 6.235402 & -0.953475 & 1.888978 \\
\hline $\mathrm{H}$ & 5.508855 & -1.302360 & 2.631575 \\
\hline $\mathrm{H}$ & 7.107316 & -1.617044 & 1.922116 \\
\hline $\mathrm{H}$ & 6.559579 & 0.055813 & 2.167200 \\
\hline $\mathrm{C}$ & 5.371115 & -2.932244 & -0.838041 \\
\hline $\mathrm{H}$ & 5.071252 & -3.653610 & -0.068552 \\
\hline $\mathrm{H}$ & 4.662065 & -2.992665 & -1.672426 \\
\hline $\mathrm{H}$ & 6.363253 & -3.219486 & -1.204048 \\
\hline $\mathrm{C}$ & 6.481672 & 0.376027 & -1.320247 \\
\hline $\mathrm{H}$ & 7.478473 & -0.028569 & -1.531089 \\
\hline $\mathrm{H}$ & 5.957174 & 0.526591 & -2.270087 \\
\hline $\mathrm{H}$ & 6.595337 & 1.352509 & -0.833470 \\
\hline $\mathrm{H}$ & 0.588737 & -3.297454 & 0.830638 \\
\hline $\mathrm{H}$ & -1.655882 & -2.805519 & 1.833153 \\
\hline $\mathrm{C}$ & -2.940836 & -0.444481 & 1.989595 \\
\hline $\mathrm{H}$ & -2.920810 & 0.379260 & 2.720796 \\
\hline $\mathrm{C}$ & -4.647650 & -1.513648 & 3.338209 \\
\hline $\mathrm{H}$ & -4.587932 & -0.725564 & 4.100825 \\
\hline $\mathrm{H}$ & -5.455743 & -1.272558 & 2.635461 \\
\hline $\mathrm{H}$ & -4.846639 & -2.474117 & 3.814433 \\
\hline $\mathrm{C}$ & -3.901951 & -0.087956 & 0.841756 \\
\hline $\mathrm{C}$ & -4.435292 & 1.095200 & 0.664341 \\
\hline $\mathrm{C}$ & -5.063230 & 2.203218 & 0.086324 \\
\hline $\mathrm{C}$ & -6.403350 & 2.572688 & 0.420666 \\
\hline $\mathrm{C}$ & -4.347998 & 3.017951 & -0.849878 \\
\hline $\mathrm{C}$ & -6.994755 & 3.690138 & -0.167023 \\
\hline $\mathrm{H}$ & -6.952669 & 1.967696 & 1.135101 \\
\hline $\mathrm{C}$ & -4.957883 & 4.130854 & -1.426552 \\
\hline $\mathrm{H}$ & -3.324849 & 2.749854 & -1.099806 \\
\hline $\mathrm{C}$ & -6.282638 & 4.477765 & -1.093534 \\
\hline $\mathrm{H}$ & -8.015452 & 3.953888 & 0.091683 \\
\hline $\mathrm{H}$ & -4.404741 & 4.734222 & -2.139326 \\
\hline $\mathrm{H}$ & -6.750638 & 5.345105 & -1.546401 \\
\hline $\mathrm{O}$ & -3.383763 & -1.654094 & 2.650370 \\
\hline $\mathrm{Sn}$ & -4.271910 & -1.614152 & -0.649695 \\
\hline $\mathrm{C}$ & -2.470727 & -2.005707 & -1.722678 \\
\hline $\mathrm{H}$ & -2.013995 & -2.930646 & -1.352434 \\
\hline $\mathrm{H}$ & -2.664990 & -2.108170 & -2.796618 \\
\hline $\mathrm{H}$ & -1.754264 & -1.189163 & -1.567353 \\
\hline $\mathrm{C}$ & -5.748194 & -0.748534 & -1.911601 \\
\hline $\mathrm{H}$ & -6.654916 & -0.514450 & -1.341807 \\
\hline $\mathrm{H}$ & -5.377750 & 0.187819 & -2.345188 \\
\hline $\mathrm{H}$ & -6.019357 & -1.425762 & -2.729381 \\
\hline $\mathrm{C}$ & -4.976979 & -3.387347 & 0.294924 \\
\hline $\mathrm{H}$ & -4.277312 & -3.665628 & 1.090280 \\
\hline $\mathrm{H}$ & -5.964812 & -3.228874 & 0.742071 \\
\hline $\mathrm{H}$ & -5.052050 & -4.211911 & -0.423036 \\
\hline
\end{tabular}


Frequencies (Top 10 out of 240)

1. $\quad 7.8053 \mathrm{~cm}-1$ (Symmetry: A)

2. $\quad 11.7073 \mathrm{~cm}-1$ (Symmetry: A)

3. $\quad 19.1070 \mathrm{~cm}-1$ (Symmetry: A)

4. $22.8668 \mathrm{~cm}-1$ (Symmetry: A)

5. $\quad 30.1874 \mathrm{~cm}-1$ (Symmetry: A)

6. $\quad 33.6406 \mathrm{~cm}-1$ (Symmetry: A)

7. $\quad 39.2589 \mathrm{~cm}-1$ (Symmetry: A)

8. $\quad 44.8722 \mathrm{~cm}-1$ (Symmetry: A)

9. $\quad 49.2035 \mathrm{~cm}-1$ (Symmetry: A)

10. $\quad 51.8600 \mathrm{~cm}-1$ (Symmetry: A)

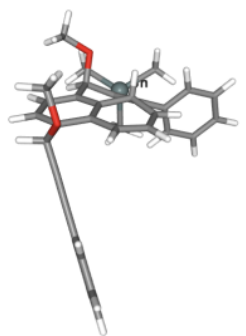

Charge

Multiplicity

0

Stoichiometry

Number of Basis Functions

Electronic Energy (Eh)

Number of Imaginary Frequencies

Mean of alpha and beta Electrons

2

C33H33O2Sn

389

$-1430.30345346$

1

125.5

Molecular Geometry in Cartesian Coordinates

$\begin{array}{lrrr}\mathrm{C} & 1.935546 & -2.878803 & -1.012075 \\ \mathrm{C} & 0.552048 & -2.747504 & -1.205613 \\ \mathrm{C} & 2.589353 & -2.141066 & -0.023779 \\ \mathrm{C} & -0.191179 & -1.894619 & -0.377175 \\ \mathrm{C} & 0.443326 & -1.159959 & 0.634045 \\ \mathrm{C} & 1.850758 & -1.258361 & 0.817441 \\ \mathrm{C} & 2.501433 & -0.427504 & 1.803100 \\ \mathrm{H} & 3.561673 & -0.548707 & 1.985381 \\ \mathrm{C} & 1.770255 & 0.589291 & 2.462645 \\ \mathrm{C} & 0.409971 & 0.734973 & 2.302156 \\ \mathrm{C} & -0.408644 & -0.271733 & 1.530991 \\ \mathrm{H} & 2.306945 & 1.284638 & 3.100865 \\ \mathrm{H} & -0.113841 & 1.545854 & 2.797135 \\ \mathrm{H} & -0.870061 & -0.950377 & 2.279770\end{array}$




\begin{tabular}{|c|c|c|c|}
\hline $\mathrm{C}$ & -1.686071 & -1.762951 & -0.553423 \\
\hline $\mathrm{H}$ & -1.952845 & -2.072510 & -1.577553 \\
\hline $\mathrm{C}$ & -2.221537 & -4.052785 & 0.123343 \\
\hline $\mathrm{H}$ & -1.190998 & -4.356954 & 0.343644 \\
\hline $\mathrm{H}$ & -2.910527 & -4.582025 & 0.782805 \\
\hline $\mathrm{H}$ & -2.452528 & -4.298173 & -0.924319 \\
\hline $\mathrm{O}$ & -2.419700 & -2.640349 & 0.374257 \\
\hline $\mathrm{C}$ & -2.203228 & -0.370922 & -0.240267 \\
\hline $\mathrm{C}$ & -1.612245 & 0.314820 & 0.765181 \\
\hline $\mathrm{C}$ & -2.188113 & 1.610669 & 1.230479 \\
\hline $\mathrm{C}$ & -2.699761 & 1.742531 & 2.536661 \\
\hline $\mathrm{C}$ & -2.289110 & 2.700559 & 0.346819 \\
\hline $\mathrm{C}$ & -3.322066 & 2.930118 & 2.941152 \\
\hline $\mathrm{H}$ & -2.637471 & 0.900727 & 3.221833 \\
\hline $\mathrm{C}$ & -2.910426 & 3.892865 & 0.750821 \\
\hline $\mathrm{H}$ & -1.871518 & 2.609095 & -0.652297 \\
\hline $\mathrm{C}$ & -3.433263 & 4.009203 & 2.047253 \\
\hline $\mathrm{H}$ & -3.727864 & 3.013189 & 3.944211 \\
\hline $\mathrm{H}$ & -2.982286 & 4.725141 & 0.057745 \\
\hline $\mathrm{H}$ & -3.917840 & 4.928182 & 2.360380 \\
\hline $\mathrm{Sn}$ & -4.094003 & 0.136530 & -1.100493 \\
\hline $\mathrm{C}$ & -3.929720 & 1.659979 & -2.588712 \\
\hline $\mathrm{H}$ & -2.957351 & 1.609771 & -3.092023 \\
\hline $\mathrm{H}$ & -4.714338 & 1.542642 & -3.345251 \\
\hline $\mathrm{H}$ & -4.034944 & 2.651070 & -2.132918 \\
\hline $\mathrm{C}$ & -4.730648 & -1.672344 & -2.036095 \\
\hline $\mathrm{H}$ & -4.174506 & -1.856653 & -2.963041 \\
\hline $\mathrm{H}$ & -4.562656 & -2.511510 & -1.350719 \\
\hline $\mathrm{H}$ & -5.797621 & -1.633903 & -2.283424 \\
\hline $\mathrm{C}$ & -5.443596 & 0.747220 & 0.424269 \\
\hline $\mathrm{H}$ & -6.474427 & 0.769073 & 0.052674 \\
\hline $\mathrm{H}$ & -5.391273 & 0.054584 & 1.271438 \\
\hline $\mathrm{H}$ & -5.178622 & 1.749029 & 0.783909 \\
\hline $\mathrm{H}$ & 0.052187 & -3.304839 & -1.993687 \\
\hline $\mathrm{H}$ & 2.510840 & -3.547948 & -1.646947 \\
\hline $\mathrm{C}$ & 4.100574 & -2.293830 & 0.114437 \\
\hline $\mathrm{H}$ & 4.416550 & -3.091569 & -0.577718 \\
\hline $\mathrm{C}$ & 3.924892 & -3.913374 & 1.914251 \\
\hline $\mathrm{H}$ & 2.840828 & -3.815357 & 2.048932 \\
\hline $\mathrm{H}$ & 4.127958 & -4.724671 & 1.200383 \\
\hline $\mathrm{H}$ & 4.397385 & -4.139046 & 2.870132 \\
\hline $\mathrm{C}$ & 4.833138 & -1.064349 & -0.233925 \\
\hline $\mathrm{C}$ & 5.453397 & -0.061172 & -0.537694 \\
\hline $\mathrm{C}$ & 6.192961 & 1.126862 & -0.875865 \\
\hline $\mathrm{C}$ & 5.700546 & 2.028528 & -1.841629 \\
\hline $\mathrm{C}$ & 7.421235 & 1.399414 & -0.238706 \\
\hline $\mathrm{C}$ & 6.427209 & 3.181300 & -2.164598 \\
\hline
\end{tabular}




$\begin{array}{lllr}\mathrm{H} & 4.753132 & 1.819374 & -2.327240 \\ \mathrm{C} & 8.142459 & 2.554935 & -0.562780 \\ \mathrm{H} & 7.797111 & 0.703525 & 0.504048 \\ \mathrm{C} & 7.649067 & 3.448647 & -1.526920 \\ \mathrm{H} & 6.041555 & 3.870834 & -2.908354 \\ \mathrm{H} & 9.085845 & 2.758347 & -0.066891 \\ \mathrm{H} & 8.209747 & 4.343128 & -1.777670 \\ \mathrm{O} & 4.512271 & -2.668940 & 1.461327\end{array}$

Frequencies (Top 10 out of 201)

1. $\quad-11.9141 \mathrm{~cm}-1$ (Symmetry: A) *

2. $\quad 14.4594 \mathrm{~cm}-1$ (Symmetry: A)

3. $20.5051 \mathrm{~cm}-1$ (Symmetry: A)

4. $\quad 30.8804 \mathrm{~cm}-1$ (Symmetry: A)

5. $\quad 42.2017 \mathrm{~cm}-1$ (Symmetry: A)

6. $\quad 48.4444 \mathrm{~cm}-1$ (Symmetry: A)

7. $\quad 57.8434 \mathrm{~cm}-1$ (Symmetry: A)

8. $\quad 60.6848 \mathrm{~cm}-1$ (Symmetry: A)

9. $\quad 64.9554 \mathrm{~cm}-1$ (Symmetry: A)

10. $71.5615 \mathrm{~cm}-1$ (Symmetry: A)

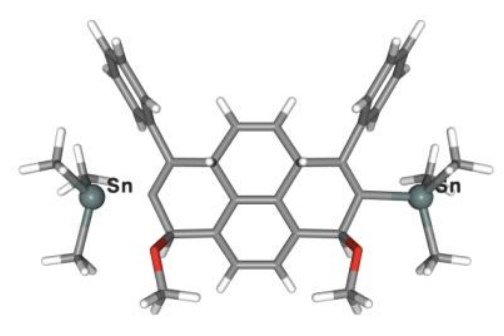

Charge

Multiplicity

Stoichiometry

Number of Basis Functions

Electronic Energy (Eh)

Number of Imaginary Frequencies

Mean of alpha and beta Electrons

0

1

$\mathrm{C} 36 \mathrm{H} 42 \mathrm{O} 2 \mathrm{Sn} 2$

442

$-1553.43223806$

0

141

Molecular Geometry in Cartesian Coordinates

$\begin{array}{lrrr}\mathrm{C} & -0.698687 & -2.946217 & 0.520236 \\ \mathrm{C} & 0.698572 & -2.946233 & 0.520260 \\ \mathrm{C} & -1.403671 & -1.816165 & 0.077048 \\ \mathrm{C} & 1.403590 & -1.816189 & 0.077104 \\ \mathrm{C} & 0.704225 & -0.663474 & -0.314445 \\ \mathrm{C} & -0.704276 & -0.663464 & -0.314479 \\ \mathrm{C} & -1.462750 & 0.600036 & -0.694205\end{array}$




\begin{tabular}{|c|c|c|c|}
\hline $\mathrm{H}$ & -1.528706 & 0.621320 & -1.798246 \\
\hline $\mathrm{C}$ & -0.669852 & 1.822900 & -0.257170 \\
\hline $\mathrm{C}$ & 0.669850 & 1.822885 & -0.257143 \\
\hline $\mathrm{C}$ & 1.462725 & 0.600010 & -0.694161 \\
\hline $\mathrm{H}$ & -1.223724 & 2.704239 & 0.051595 \\
\hline $\mathrm{H}$ & 1.223735 & 2.704208 & 0.051649 \\
\hline $\mathrm{H}$ & 1.528686 & 0.621304 & -1.798206 \\
\hline $\mathrm{C}$ & 2.910549 & -1.850927 & -0.006785 \\
\hline $\mathrm{H}$ & 3.292379 & -2.587307 & 0.720861 \\
\hline $\mathrm{C}$ & 3.036887 & -3.662786 & -1.658107 \\
\hline $\mathrm{H}$ & 1.957025 & -3.797989 & -1.794453 \\
\hline $\mathrm{H}$ & 3.555892 & -3.893458 & -2.589478 \\
\hline $\mathrm{H}$ & 3.387993 & -4.339909 & -0.864315 \\
\hline $\mathrm{O}$ & 3.360268 & -2.287920 & -1.341308 \\
\hline $\mathrm{C}$ & 3.579374 & -0.503298 & 0.170716 \\
\hline $\mathrm{C}$ & 2.906362 & 0.615916 & -0.183514 \\
\hline $\mathrm{C}$ & 3.600725 & 1.938776 & -0.191977 \\
\hline $\mathrm{C}$ & 3.689296 & 2.693968 & -1.378043 \\
\hline $\mathrm{C}$ & 4.213019 & 2.432678 & 0.974636 \\
\hline $\mathrm{C}$ & 4.408981 & 3.895228 & -1.405228 \\
\hline $\mathrm{H}$ & 3.217240 & 2.324667 & -2.284565 \\
\hline $\mathrm{C}$ & 4.931165 & 3.638256 & 0.951079 \\
\hline $\mathrm{H}$ & 4.110971 & 1.874442 & 1.901251 \\
\hline $\mathrm{C}$ & 5.038650 & 4.369055 & -0.241601 \\
\hline $\mathrm{H}$ & 4.485304 & 4.457381 & -2.330365 \\
\hline $\mathrm{H}$ & 5.398595 & 4.005221 & 1.859449 \\
\hline $\mathrm{H}$ & 5.597929 & 5.298577 & -0.264447 \\
\hline Sn & 5.699000 & -0.592394 & 0.471499 \\
\hline $\mathrm{C}$ & 6.297245 & 0.075111 & 2.412105 \\
\hline $\mathrm{H}$ & 5.501441 & -0.087587 & 3.148283 \\
\hline $\mathrm{H}$ & 7.186774 & -0.474279 & 2.742369 \\
\hline $\mathrm{H}$ & 6.539071 & 1.143872 & 2.392115 \\
\hline $\mathrm{C}$ & 6.194028 & -2.660966 & 0.321073 \\
\hline $\mathrm{H}$ & 5.797274 & -3.225839 & 1.173727 \\
\hline $\mathrm{H}$ & 5.766587 & -3.068124 & -0.601450 \\
\hline $\mathrm{H}$ & 7.280871 & -2.801925 & 0.299973 \\
\hline $\mathrm{C}$ & 6.669184 & 0.565010 & -1.025166 \\
\hline $\mathrm{H}$ & 7.744079 & 0.353164 & -1.053981 \\
\hline $\mathrm{H}$ & 6.240721 & 0.339614 & -2.007977 \\
\hline $\mathrm{H}$ & 6.526871 & 1.634278 & -0.825351 \\
\hline $\mathrm{H}$ & 1.248157 & -3.821537 & 0.858226 \\
\hline $\mathrm{H}$ & -1.248287 & -3.821514 & 0.858190 \\
\hline $\mathrm{C}$ & -2.910635 & -1.850860 & -0.006796 \\
\hline $\mathrm{H}$ & -3.292458 & -2.587257 & 0.720833 \\
\hline $\mathrm{C}$ & -3.036945 & -3.662537 & -1.658397 \\
\hline $\mathrm{H}$ & -1.957155 & -3.797432 & -1.795595 \\
\hline $\mathrm{H}$ & -3.387220 & -4.339808 & -0.864367 \\
\hline
\end{tabular}




$\begin{array}{lrrr}\mathrm{H} & -3.556607 & -3.893329 & -2.589373 \\ \mathrm{C} & -3.579408 & -0.503204 & 0.170770 \\ \mathrm{C} & -2.906399 & 0.615991 & -0.183544 \\ \mathrm{C} & -3.600667 & 1.938900 & -0.192117 \\ \mathrm{C} & -4.213093 & 2.432811 & 0.974426 \\ \mathrm{C} & -3.688970 & 2.694175 & -1.378154 \\ \mathrm{C} & -4.931084 & 3.638478 & 0.950837 \\ \mathrm{H} & -4.111240 & 1.874516 & 1.901027 \\ \mathrm{C} & -4.408492 & 3.895533 & -1.405366 \\ \mathrm{H} & -3.216825 & 2.324883 & -2.284633 \\ \mathrm{C} & -5.038281 & 4.369373 & -0.241809 \\ \mathrm{H} & -5.398610 & 4.005442 & 1.859158 \\ \mathrm{H} & -4.484594 & 4.457759 & -2.330479 \\ \mathrm{H} & -5.597435 & 5.298968 & -0.264687 \\ \mathrm{O} & -3.360485 & -2.287778 & -1.341306 \\ \mathrm{Sn} & -5.699045 & -0.592402 & 0.471565 \\ \mathrm{C} & -6.193900 & -2.661056 & 0.321608 \\ \mathrm{H} & -5.797961 & -3.225577 & 1.174866 \\ \mathrm{H} & -7.280728 & -2.801995 & 0.299552 \\ \mathrm{H} & -5.765635 & -3.068606 & -0.600360 \\ \mathrm{C} & -6.669420 & 0.564513 & -1.025329 \\ \mathrm{H} & -6.527009 & 1.633829 & -0.825873 \\ \mathrm{H} & -6.241159 & 0.338768 & -2.008143 \\ \mathrm{H} & -7.744332 & 0.352718 & -1.053861 \\ \mathrm{C} & -6.297309 & 0.075496 & 2.412024 \\ \mathrm{H} & -5.501557 & -0.087244 & 3.148252 \\ \mathrm{H} & -6.538944 & 1.144300 & 2.391880 \\ \mathrm{H} & -7.186939 & -0.473701 & 2.742331\end{array}$

Frequencies (Top 10 out of 240)

1. $\quad 18.8313 \mathrm{~cm}-1$ (Symmetry: A)

2. $\quad 19.0648 \mathrm{~cm}-1$ (Symmetry: A)

3. $\quad 34.2162 \mathrm{~cm}-1$ (Symmetry: A)

4. $\quad 39.8885 \mathrm{~cm}-1$ (Symmetry: A)

5. $44.3497 \mathrm{~cm}-1$ (Symmetry: A)

6. $\quad 45.2453 \mathrm{~cm}-1$ (Symmetry: A)

7. $\quad 48.2261 \mathrm{~cm}-1$ (Symmetry: A)

8. $\quad 55.0820 \mathrm{~cm}-1$ (Symmetry: A)

9. $\quad 57.3922 \mathrm{~cm}-1$ (Symmetry: A)

10. $57.6742 \mathrm{~cm}-1$ (Symmetry: A) 


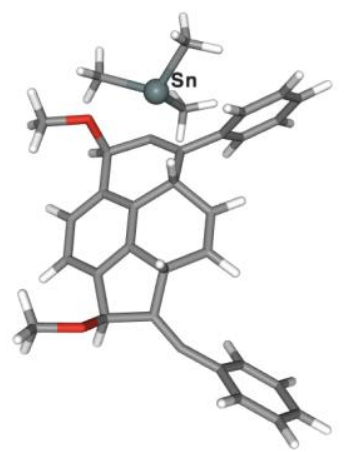

Charge

Multiplicity

Stoichiometry

Number of Basis Functions

Electronic Energy (Eh)

Number of Imaginary Frequencies

Mean of alpha and beta Electrons
0

2

$\mathrm{C} 33 \mathrm{H} 33 \mathrm{O} 2 \mathrm{Sn}$

389

$-1430.30908566$

0

125.5

Molecular Geometry in Cartesian Coordinates

$\begin{array}{lrrr}\mathrm{C} & 1.639641 & -3.399396 & -0.926698 \\ \mathrm{C} & 0.246518 & -3.197025 & -0.894961 \\ \mathrm{C} & 2.457613 & -2.494087 & -0.244664 \\ \mathrm{C} & -0.320387 & -2.101878 & -0.217417 \\ \mathrm{C} & 0.510384 & -1.196711 & 0.463798 \\ \mathrm{C} & 1.878402 & -1.435597 & 0.463199 \\ \mathrm{C} & 2.906166 & -0.475759 & 1.007746 \\ \mathrm{H} & 3.131671 & -0.705162 & 2.060124 \\ \mathrm{C} & 2.388498 & 0.939245 & 0.891972 \\ \mathrm{C} & 1.066985 & 1.193986 & 0.913504 \\ \mathrm{C} & -0.000380 & 0.102105 & 1.056334 \\ \mathrm{H} & 3.111986 & 1.747029 & 0.804592 \\ \mathrm{H} & 0.711209 & 2.218628 & 0.847700 \\ \mathrm{H} & -0.166085 & -0.055455 & 2.137376 \\ \mathrm{C} & -1.809098 & -1.851955 & -0.229107 \\ \mathrm{H} & -2.247249 & -2.323067 & -1.125893 \\ \mathrm{C} & -2.428961 & -3.899482 & 0.960997 \\ \mathrm{H} & -1.407913 & -4.268922 & 1.114800 \\ \mathrm{H} & -3.061071 & -4.213159 & 1.792714 \\ \mathrm{H} & -2.825227 & -4.312638 & 0.020833 \\ \mathrm{O} & -2.468100 & -2.452441 & 0.942149 \\ \mathrm{C} & -2.185383 & -0.381492 & -0.146784 \\ \mathrm{C} & -1.360134 & 0.498697 & 0.470591 \\ \mathrm{C} & -1.796341 & 1.908265 & 0.704183 \\ \mathrm{C} & -1.867471 & 2.426590 & 2.012310 \\ \mathrm{C} & -2.171169 & 2.731749 & -0.373510\end{array}$




\begin{tabular}{|c|c|c|c|}
\hline $\mathrm{C}$ & -2.341591 & 3.724871 & 2.239187 \\
\hline $\mathrm{H}$ & -1.577541 & 1.801725 & 2.852817 \\
\hline $\mathrm{C}$ & -2.644043 & 4.033970 & -0.149208 \\
\hline $\mathrm{H}$ & -2.075222 & 2.350220 & -1.386394 \\
\hline $\mathrm{C}$ & -2.738116 & 4.531960 & 1.158862 \\
\hline $\mathrm{H}$ & -2.408980 & 4.105540 & 3.253181 \\
\hline $\mathrm{H}$ & -2.930800 & 4.655739 & -0.991557 \\
\hline $\mathrm{H}$ & -3.107497 & 5.536763 & 1.335831 \\
\hline $\mathrm{Sn}$ & -4.238627 & 0.030100 & -0.610555 \\
\hline $\mathrm{C}$ & -4.471504 & 1.171364 & -2.403333 \\
\hline $\mathrm{H}$ & -3.636005 & 0.998090 & -3.091612 \\
\hline $\mathrm{H}$ & -5.399362 & 0.888070 & -2.913890 \\
\hline $\mathrm{H}$ & -4.516485 & 2.241905 & -2.173316 \\
\hline $\mathrm{C}$ & -5.130887 & -1.877453 & -0.944784 \\
\hline $\mathrm{H}$ & -4.766216 & -2.328106 & -1.876111 \\
\hline $\mathrm{H}$ & -4.885588 & -2.543347 & -0.110592 \\
\hline $\mathrm{H}$ & -6.221196 & -1.788976 & -1.017053 \\
\hline $\mathrm{C}$ & -5.143328 & 1.041720 & 1.025818 \\
\hline $\mathrm{H}$ & -6.235441 & 1.041380 & 0.933403 \\
\hline $\mathrm{H}$ & -4.870937 & 0.547485 & 1.964785 \\
\hline $\mathrm{H}$ & -4.794936 & 2.080829 & 1.069823 \\
\hline $\mathrm{H}$ & -0.408180 & -3.875730 & -1.437228 \\
\hline $\mathrm{H}$ & 2.062919 & -4.217805 & -1.502418 \\
\hline $\mathrm{C}$ & 3.973735 & -2.355144 & -0.198922 \\
\hline $\mathrm{H}$ & 4.468103 & -2.622699 & -1.142029 \\
\hline $\mathrm{C}$ & 4.410224 & -4.551656 & 0.743071 \\
\hline $\mathrm{H}$ & 3.357352 & -4.846228 & 0.836533 \\
\hline $\mathrm{H}$ & 4.795722 & -4.891067 & -0.229635 \\
\hline $\mathrm{H}$ & 4.990590 & -5.009599 & 1.544429 \\
\hline $\mathrm{C}$ & 4.139909 & -0.872300 & 0.164452 \\
\hline $\mathrm{C}$ & 5.099817 & -0.076813 & -0.242003 \\
\hline $\mathrm{C}$ & 5.820413 & 1.104005 & -0.479005 \\
\hline $\mathrm{C}$ & 5.443496 & 1.967389 & -1.553844 \\
\hline $\mathrm{C}$ & 6.959330 & 1.457003 & 0.306002 \\
\hline $\mathrm{C}$ & 6.167223 & 3.130174 & -1.815717 \\
\hline $\mathrm{H}$ & 4.580646 & 1.703291 & -2.157287 \\
\hline $\mathrm{C}$ & 7.672559 & 2.624130 & 0.029827 \\
\hline $\mathrm{H}$ & 7.258169 & 0.803244 & 1.118720 \\
\hline $\mathrm{C}$ & 7.285623 & 3.468162 & -1.029073 \\
\hline $\mathrm{H}$ & 5.864690 & 3.778390 & -2.631803 \\
\hline $\mathrm{H}$ & 8.533937 & 2.882599 & 0.637401 \\
\hline $\mathrm{H}$ & 7.846389 & 4.372824 & -1.238594 \\
\hline $\mathrm{O}$ & 4.569778 & -3.121137 & 0.889246 \\
\hline
\end{tabular}

Frequencies (Top 10 out of 201) 
1. $\quad 10.2460 \mathrm{~cm}-1$ (Symmetry: A)

2. $\quad 18.2051 \mathrm{~cm}-1$ (Symmetry: A)

3. $\quad 26.4027 \mathrm{~cm}-1$ (Symmetry: A)

4. $\quad 36.2878 \mathrm{~cm}-1$ (Symmetry: A)

5. $\quad 39.9893 \mathrm{~cm}-1$ (Symmetry: A)

6. $\quad 46.5331 \mathrm{~cm}-1$ (Symmetry: A)

7. $\quad 52.2713 \mathrm{~cm}-1$ (Symmetry: A)

8. $\quad 53.0125 \mathrm{~cm}-1$ (Symmetry: A)

9. $\quad 58.0923 \mathrm{~cm}-1$ (Symmetry: A)

10. $\quad 65.8059 \mathrm{~cm}-1$ (Symmetry: A)

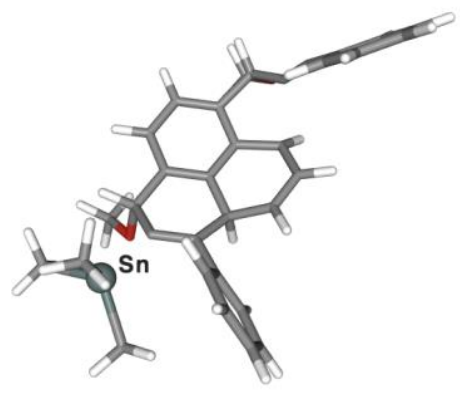

Charge

Multiplicity

Stoichiometry

Number of Basis Functions

0

2

Electronic Energy (Eh)

Number of Imaginary Frequencies

Mean of alpha and beta Electrons

C33H33O2Sn

389

$-1430.26955084$

1

125.5

Molecular Geometry in Cartesian Coordinates

$\begin{array}{lrrr}\mathrm{C} & -1.801182 & 3.352493 & -1.010755 \\ \mathrm{C} & -0.408357 & 3.157945 & -1.044326 \\ \mathrm{C} & -2.572843 & 2.544219 & -0.176529 \\ \mathrm{C} & 0.193541 & 2.142719 & -0.280998 \\ \mathrm{C} & -0.587678 & 1.333907 & 0.561217 \\ \mathrm{C} & -1.966449 & 1.577047 & 0.648477 \\ \mathrm{C} & -2.874878 & 0.696644 & 1.395286 \\ \mathrm{H} & -3.598653 & 1.134124 & 2.079933 \\ \mathrm{C} & -2.363511 & -0.644668 & 1.650862 \\ \mathrm{C} & -1.037907 & -0.924040 & 1.551258 \\ \mathrm{C} & 0.011620 & 0.154682 & 1.304601 \\ \mathrm{H} & -3.065562 & -1.424444 & 1.935370 \\ \mathrm{H} & -0.679730 & -1.931982 & 1.739164\end{array}$




\begin{tabular}{|c|c|c|c|}
\hline $\mathrm{H}$ & 0.331313 & 0.519052 & 2.300263 \\
\hline $\mathrm{C}$ & 1.679464 & 1.884918 & -0.363056 \\
\hline $\mathrm{H}$ & 2.058022 & 2.270346 & -1.324892 \\
\hline $\mathrm{C}$ & 2.364829 & 4.029435 & 0.596311 \\
\hline $\mathrm{H}$ & 1.356335 & 4.411954 & 0.794537 \\
\hline $\mathrm{H}$ & 3.058456 & 4.416865 & 1.343591 \\
\hline $\mathrm{H}$ & 2.683617 & 4.355860 & -0.405466 \\
\hline $\mathrm{O}$ & 2.411313 & 2.586304 & 0.704590 \\
\hline $\mathrm{C}$ & 2.056889 & 0.426690 & -0.167951 \\
\hline $\mathrm{C}$ & 1.291986 & -0.355929 & 0.629738 \\
\hline $\mathrm{C}$ & 1.741625 & -1.733447 & 0.991050 \\
\hline $\mathrm{C}$ & 1.972709 & -2.075133 & 2.338294 \\
\hline $\mathrm{C}$ & 1.984733 & -2.696170 & -0.005673 \\
\hline $\mathrm{C}$ & 2.469101 & -3.339632 & 2.677896 \\
\hline $\mathrm{H}$ & 1.789370 & -1.337664 & 3.115411 \\
\hline $\mathrm{C}$ & 2.479347 & -3.965699 & 0.332515 \\
\hline $\mathrm{H}$ & 1.772266 & -2.448615 & -1.042270 \\
\hline $\mathrm{C}$ & 2.729631 & -4.288861 & 1.674394 \\
\hline $\mathrm{H}$ & 2.660073 & -3.583253 & 3.718054 \\
\hline $\mathrm{H}$ & 2.662427 & -4.698405 & -0.447242 \\
\hline $\mathrm{H}$ & 3.115338 & -5.268235 & 1.937846 \\
\hline $\mathrm{Sn}$ & 4.046263 & -0.078837 & -0.782256 \\
\hline $\mathrm{C}$ & 4.084463 & -1.422402 & -2.444178 \\
\hline $\mathrm{H}$ & 3.183035 & -1.313599 & -3.058570 \\
\hline $\mathrm{H}$ & 4.956919 & -1.220908 & -3.076770 \\
\hline $\mathrm{H}$ & 4.142945 & -2.459220 & -2.093729 \\
\hline $\mathrm{C}$ & 4.904824 & 1.769302 & -1.410730 \\
\hline $\mathrm{H}$ & 4.488908 & 2.092545 & -2.372711 \\
\hline $\mathrm{H}$ & 4.698528 & 2.539416 & -0.658900 \\
\hline $\mathrm{H}$ & 5.990809 & 1.675198 & -1.526925 \\
\hline $\mathrm{C}$ & 5.100743 & -0.924930 & 0.858728 \\
\hline $\mathrm{H}$ & 4.741188 & -1.943202 & 1.051763 \\
\hline $\mathrm{H}$ & 6.177633 & -0.964220 & 0.659210 \\
\hline $\mathrm{H}$ & 4.932723 & -0.325128 & 1.760004 \\
\hline $\mathrm{H}$ & 0.210020 & 3.764618 & -1.701610 \\
\hline $\mathrm{H}$ & -2.271292 & 4.085985 & -1.660610 \\
\hline $\mathrm{C}$ & -4.084182 & 2.430809 & -0.176387 \\
\hline $\mathrm{H}$ & -4.524382 & 2.850830 & -1.091948 \\
\hline $\mathrm{C}$ & -4.467979 & 4.501081 & 1.024693 \\
\hline $\mathrm{H}$ & -3.404601 & 4.728165 & 1.169453 \\
\hline $\mathrm{H}$ & -4.816528 & 4.980295 & 0.098351 \\
\hline $\mathrm{H}$ & -5.044691 & 4.877536 & 1.869547 \\
\hline $\mathrm{C}$ & -4.384835 & 0.963072 & -0.064384 \\
\hline $\mathrm{C}$ & -4.977035 & -0.065750 & -0.449422 \\
\hline $\mathrm{C}$ & -5.448302 & -1.376086 & -0.739393 \\
\hline $\mathrm{C}$ & -4.661911 & -2.246956 & -1.536048 \\
\hline $\mathrm{C}$ & -6.697465 & -1.833881 & -0.251493 \\
\hline
\end{tabular}




$\begin{array}{llll}\text { C } & -5.116157 & -3.534761 & -1.834083 \\ \text { H } & -3.702575 & -1.898243 & -1.904474 \\ \text { C } & -7.142646 & -3.123999 & -0.555411 \\ \text { H } & -7.301789 & -1.169441 & 0.357352 \\ \text { C } & -6.357672 & -3.980053 & -1.347826 \\ \text { H } & -4.504879 & -4.192801 & -2.443178 \\ \text { H } & -8.100794 & -3.465426 & -0.177188 \\ \text { H } & -6.708498 & -4.979558 & -1.582342 \\ \text { O } & -4.697089 & 3.072194 & 0.981872\end{array}$

Frequencies (Top 10 out of 201)

1. $-647.1418 \mathrm{~cm}-1$ (Symmetry: A) *

2. $\quad 16.9548 \mathrm{~cm}-1$ (Symmetry: A)

3. $\quad 19.5315 \mathrm{~cm}-1$ (Symmetry: A)

4. $29.2156 \mathrm{~cm}-1$ (Symmetry: A)

5. $\quad 36.9500 \mathrm{~cm}-1$ (Symmetry: A)

6. $42.6228 \mathrm{~cm}-1$ (Symmetry: A)

7. $\quad 49.5782 \mathrm{~cm}-1$ (Symmetry: A)

8. $\quad 57.0197 \mathrm{~cm}-1$ (Symmetry: A)

9. $\quad 61.2422 \mathrm{~cm}-1$ (Symmetry: A)

10. $63.7192 \mathrm{~cm}-1$ (Symmetry: A)

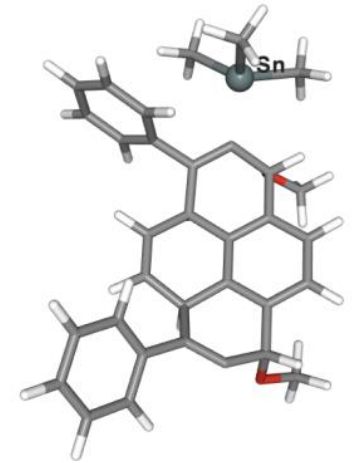

Charge

Multiplicity

0

Stoichiometry

Number of Basis Functions

C33H33O2Sn

389

Electronic Energy (Eh)

$-1430.30223147$

Number of Imaginary Frequencies

Mean of alpha and beta Electrons

125.5

Molecular Geometry in Cartesian Coordinates

C $\quad 1.845821 \quad-3.274991 \quad-1.030654$ 


\begin{tabular}{|c|c|c|c|}
\hline $\mathrm{C}$ & 0.456538 & -3.143234 & -0.911316 \\
\hline $\mathrm{C}$ & 2.685495 & -2.312736 & -0.461455 \\
\hline $\mathrm{C}$ & -0.085048 & -2.060368 & -0.210184 \\
\hline $\mathrm{C}$ & 0.754926 & -1.085197 & 0.363156 \\
\hline $\mathrm{C}$ & 2.148095 & -1.200398 & 0.221914 \\
\hline $\mathrm{C}$ & 3.103313 & -0.138100 & 0.762526 \\
\hline $\mathrm{H}$ & 3.537195 & -0.537304 & 1.695466 \\
\hline $\mathrm{C}$ & 2.392363 & 1.145351 & 1.112600 \\
\hline $\mathrm{C}$ & 1.062808 & 1.245684 & 1.254508 \\
\hline $\mathrm{C}$ & 0.109470 & 0.080200 & 1.100035 \\
\hline $\mathrm{H}$ & 3.017838 & 2.011832 & 1.306354 \\
\hline $\mathrm{H}$ & 0.632254 & 2.199426 & 1.544148 \\
\hline $\mathrm{H}$ & -0.131695 & -0.283781 & 2.115964 \\
\hline $\mathrm{C}$ & -1.581372 & -1.919278 & -0.058963 \\
\hline $\mathrm{H}$ & -2.077312 & -2.520096 & -0.839555 \\
\hline $\mathrm{C}$ & -1.870546 & -3.855211 & 1.407592 \\
\hline $\mathrm{H}$ & -0.808969 & -4.127338 & 1.454673 \\
\hline $\mathrm{H}$ & -2.359600 & -4.118310 & 2.346259 \\
\hline $\mathrm{H}$ & -2.345903 & -4.401666 & 0.579022 \\
\hline $\mathrm{O}$ & -2.042498 & -2.426650 & 1.243720 \\
\hline $\mathrm{C}$ & -2.047934 & -0.475887 & -0.087966 \\
\hline $\mathrm{C}$ & -1.252022 & 0.456885 & 0.484580 \\
\hline $\mathrm{C}$ & -1.736088 & 1.861376 & 0.637365 \\
\hline $\mathrm{C}$ & -2.064889 & 2.628688 & -0.495047 \\
\hline $\mathrm{C}$ & -1.925654 & 2.420314 & 1.916933 \\
\hline $\mathrm{C}$ & -2.592206 & 3.922488 & -0.353435 \\
\hline $\mathrm{H}$ & -1.896810 & 2.210476 & -1.484098 \\
\hline $\mathrm{C}$ & -2.453294 & 3.709665 & 2.060852 \\
\hline $\mathrm{H}$ & -1.683992 & 1.830661 & 2.797806 \\
\hline $\mathrm{C}$ & -2.791878 & 4.465102 & 0.924532 \\
\hline $\mathrm{H}$ & -2.842370 & 4.502488 & -1.236272 \\
\hline $\mathrm{H}$ & -2.608046 & 4.122525 & 3.052520 \\
\hline $\mathrm{H}$ & -3.202442 & 5.463254 & 1.036437 \\
\hline $\mathrm{Sn}$ & -4.101175 & -0.167220 & -0.597902 \\
\hline $\mathrm{C}$ & -4.334050 & 0.785432 & -2.494869 \\
\hline $\mathrm{H}$ & -3.502289 & 0.534807 & -3.163041 \\
\hline $\mathrm{H}$ & -5.267605 & 0.467766 & -2.973507 \\
\hline $\mathrm{H}$ & -4.363617 & 1.873767 & -2.369378 \\
\hline $\mathrm{C}$ & -4.903669 & -2.140908 & -0.707597 \\
\hline $\mathrm{H}$ & -4.606916 & -2.640417 & -1.637515 \\
\hline $\mathrm{H}$ & -4.534297 & -2.727053 & 0.142319 \\
\hline $\mathrm{H}$ & -5.998642 & -2.122650 & -0.667147 \\
\hline $\mathrm{C}$ & -5.058769 & 0.984785 & 0.910877 \\
\hline $\mathrm{H}$ & -6.149485 & 0.915546 & 0.826644 \\
\hline $\mathrm{H}$ & -4.760705 & 0.627898 & 1.902747 \\
\hline $\mathrm{H}$ & -4.766089 & 2.038541 & 0.824381 \\
\hline $\mathrm{H}$ & -0.206730 & -3.878222 & -1.360655 \\
\hline
\end{tabular}




$\begin{array}{lrrr}\mathrm{H} & 2.276416 & -4.116458 & -1.567566 \\ \mathrm{C} & 4.199142 & -2.452335 & -0.566110 \\ \mathrm{H} & 4.455043 & -3.114905 & -1.406337 \\ \mathrm{C} & 4.364409 & -4.374172 & 0.925952 \\ \mathrm{H} & 3.296901 & -4.435265 & 1.170533 \\ \mathrm{H} & 4.572821 & -5.018850 & 0.059411 \\ \mathrm{H} & 4.956617 & -4.707422 & 1.778629 \\ \mathrm{C} & 4.783212 & -1.092015 & -0.729930 \\ \mathrm{C} & 4.327093 & 0.032531 & -0.178353 \\ \mathrm{C} & 5.019014 & 1.336398 & -0.385098 \\ \mathrm{C} & 6.401832 & 1.431192 & -0.136390 \\ \mathrm{C} & 4.337133 & 2.463626 & -0.884584 \\ \mathrm{C} & 7.088092 & 2.631989 & -0.367450 \\ \mathrm{H} & 6.926492 & 0.559066 & 0.242278 \\ \mathrm{C} & 5.023286 & 3.662794 & -1.117864 \\ \mathrm{H} & 3.275945 & 2.393131 & -1.104470 \\ \mathrm{C} & 6.400365 & 3.752503 & -0.856569 \\ \mathrm{H} & 8.153051 & 2.691872 & -0.167380 \\ \mathrm{H} & 4.487658 & 4.522036 & -1.508609 \\ \mathrm{H} & 6.929540 & 4.682784 & -1.036101 \\ \mathrm{O} & 4.770573 & -3.009620 & 0.661729\end{array}$

Frequencies (Top 10 out of 201)

1. $\quad 11.6765 \mathrm{~cm}-1$ (Symmetry: A)

2. $\quad 17.9467 \mathrm{~cm}-1$ (Symmetry: A)

3. $\quad 32.5774 \mathrm{~cm}-1$ (Symmetry: A)

4. $\quad 41.8636 \mathrm{~cm}-1$ (Symmetry: A)

5. $\quad 45.6263 \mathrm{~cm}-1$ (Symmetry: A)

6. $\quad 51.5881 \mathrm{~cm}-1$ (Symmetry: A)

7. $\quad 55.2519 \mathrm{~cm}-1$ (Symmetry: A)

8. $\quad 61.6713 \mathrm{~cm}-1$ (Symmetry: A)

9. $\quad 64.6917 \mathrm{~cm}-1$ (Symmetry: A)

10. $\quad 72.2755 \mathrm{~cm}-1$ (Symmetry: A)

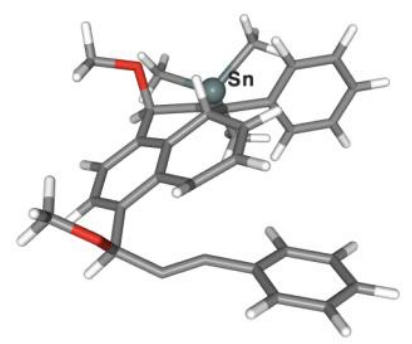

Charge

0

Multiplicity

2

Stoichiometry

$\mathrm{C} 33 \mathrm{H} 33 \mathrm{O} 2 \mathrm{Sn}$ 
Number of Basis Functions

389

Electronic Energy (Eh)

$-1430.26176471$

Number of Imaginary Frequencies

Mean of alpha and beta Electrons

125.5

Molecular Geometry in Cartesian Coordinates

\begin{tabular}{|c|c|c|c|}
\hline $\mathrm{C}$ & 1.941477 & 3.362916 & 1.038137 \\
\hline $\mathrm{C}$ & 0.550326 & 3.272456 & 0.872883 \\
\hline $\mathrm{C}$ & 2.780706 & 2.431313 & 0.424218 \\
\hline $\mathrm{C}$ & 0.004160 & 2.236470 & 0.105720 \\
\hline $\mathrm{C}$ & 0.840566 & 1.303934 & -0.532177 \\
\hline $\mathrm{C}$ & 2.240845 & 1.407507 & -0.397500 \\
\hline $\mathrm{C}$ & 3.130587 & 0.397641 & -0.994113 \\
\hline $\mathrm{H}$ & 4.061241 & 0.748340 & -1.432419 \\
\hline $\mathrm{C}$ & 2.508853 & -0.783295 & -1.567278 \\
\hline $\mathrm{C}$ & 1.162822 & -0.927156 & -1.667588 \\
\hline $\mathrm{C}$ & 0.191467 & 0.180253 & -1.327955 \\
\hline $\mathrm{H}$ & 3.161146 & -1.577069 & -1.921267 \\
\hline $\mathrm{H}$ & 0.752463 & -1.839963 & -2.088168 \\
\hline $\mathrm{H}$ & -0.143711 & 0.622519 & -2.285863 \\
\hline $\mathrm{C}$ & -1.492552 & 2.067959 & -0.027441 \\
\hline $\mathrm{H}$ & -1.990406 & 2.641801 & 0.771580 \\
\hline $\mathrm{C}$ & -1.835183 & 4.013227 & -1.461494 \\
\hline $\mathrm{H}$ & -0.777294 & 4.297620 & -1.518142 \\
\hline $\mathrm{H}$ & -2.339298 & 4.282564 & -2.390297 \\
\hline $\mathrm{H}$ & -2.305780 & 4.543502 & -0.619985 \\
\hline $\mathrm{O}$ & -1.988856 & 2.580642 & -1.312862 \\
\hline $\mathrm{C}$ & -1.908658 & 0.606156 & -0.004599 \\
\hline $\mathrm{C}$ & -1.112415 & -0.282630 & -0.643553 \\
\hline $\mathrm{C}$ & -1.526609 & -1.710488 & -0.774257 \\
\hline $\mathrm{C}$ & -1.705461 & -2.503011 & 0.374285 \\
\hline $\mathrm{C}$ & -1.801245 & -2.270124 & -2.037563 \\
\hline $\mathrm{C}$ & -2.169538 & -3.823746 & 0.265691 \\
\hline $\mathrm{H}$ & -1.474455 & -2.080448 & 1.348709 \\
\hline $\mathrm{C}$ & -2.265871 & -3.586750 & -2.148314 \\
\hline $\mathrm{H}$ & -1.680300 & -1.659305 & -2.928890 \\
\hline $\mathrm{C}$ & -2.455098 & -4.367794 & -0.995164 \\
\hline $\mathrm{H}$ & -2.306394 & -4.422442 & 1.160829 \\
\hline $\mathrm{H}$ & -2.489017 & -4.000192 & -3.126580 \\
\hline $\mathrm{H}$ & -2.818826 & -5.386462 & -1.080714 \\
\hline $\mathrm{Sn}$ & -3.895069 & 0.184964 & 0.661334 \\
\hline $\mathrm{C}$ & -3.918361 & -0.758865 & 2.576610 \\
\hline $\mathrm{H}$ & -4.829227 & -0.493720 & 3.125580 \\
\hline $\mathrm{H}$ & -3.890473 & -1.848553 & 2.461239 \\
\hline $\mathrm{H}$ & -3.053892 & -0.451243 & 3.175963 \\
\hline
\end{tabular}




$\begin{array}{lrrr}\mathrm{C} & -4.796598 & 2.112482 & 0.814323 \\ \mathrm{H} & -4.478463 & 2.629928 & 1.727128 \\ \mathrm{H} & -4.505669 & 2.716942 & -0.053318 \\ \mathrm{H} & -5.889624 & 2.035119 & 0.831702 \\ \mathrm{C} & -4.908886 & -1.034774 & -0.754541 \\ \mathrm{H} & -5.984729 & -1.062242 & -0.546458 \\ \mathrm{H} & -4.758546 & -0.644955 & -1.767251 \\ \mathrm{H} & -4.518330 & -2.059288 & -0.720210 \\ \mathrm{H} & -0.108410 & 3.984812 & 1.363293 \\ \mathrm{H} & 2.368075 & 4.136140 & 1.672117 \\ \mathrm{C} & 4.270036 & 2.399058 & 0.752644 \\ \mathrm{H} & 4.473918 & 3.096265 & 1.578454 \\ \mathrm{C} & 4.896423 & 4.126724 & -0.828568 \\ \mathrm{H} & 3.891238 & 4.258817 & -1.246897 \\ \mathrm{H} & 5.035148 & 4.831267 & 0.004157 \\ \mathrm{H} & 5.645648 & 4.317259 & -1.597120 \\ \mathrm{C} & 4.620045 & 0.995297 & 1.110707 \\ \mathrm{C} & 4.336030 & -0.164129 & 0.690444 \\ \mathrm{C} & 4.501759 & -1.607846 & 0.715029 \\ \mathrm{C} & 5.767081 & -2.181057 & 0.474427 \\ \mathrm{C} & 3.394960 & -2.446749 & 0.955751 \\ \mathrm{C} & 5.921168 & -3.573855 & 0.479139 \\ \mathrm{H} & 6.614423 & -1.528838 & 0.289199 \\ \mathrm{C} & 3.553896 & -3.837965 & 0.963102 \\ \mathrm{H} & 2.420348 & -2.001068 & 1.130043 \\ \mathrm{C} & 4.816373 & -4.405337 & 0.723201 \\ \mathrm{H} & 6.898239 & -4.009314 & 0.296498 \\ \mathrm{H} & 2.697309 & -4.476599 & 1.152219 \\ \mathrm{H} & 4.937899 & -5.483742 & 0.726511 \\ \mathrm{O} & 5.106429 & 2.762787 & -0.387585\end{array}$

Frequencies (Top 10 out of 201)

1. $-697.0640 \mathrm{~cm}-1$ (Symmetry: A) *

2. $\quad 14.0691 \mathrm{~cm}-1$ (Symmetry: A)

3. $\quad 18.6538 \mathrm{~cm}-1$ (Symmetry: A)

4. $\quad 30.3177 \mathrm{~cm}-1$ (Symmetry: A)

5. $\quad 39.3265 \mathrm{~cm}-1$ (Symmetry: A)

6. $\quad 42.2863 \mathrm{~cm}-1$ (Symmetry: A)

7. $\quad 53.8962 \mathrm{~cm}-1$ (Symmetry: A)

8. $\quad 57.2312 \mathrm{~cm}-1$ (Symmetry: A)

9. $\quad 63.5386 \mathrm{~cm}-1$ (Symmetry: A)

10. $68.3057 \mathrm{~cm}-1$ (Symmetry: A) 


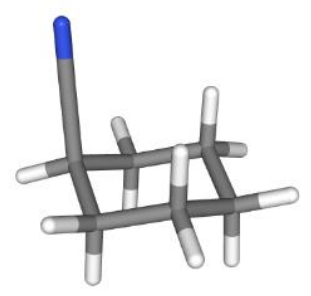

Charge

Multiplicity

Stoichiometry

Number of Basis Functions

Electronic Energy (Eh)

Number of Imaginary Frequencies

Mean of alpha and beta Electrons
0

1

C7H11N

94

$-327.919736846$

0

30

Molecular Geometry in Cartesian Coordinates

$\begin{array}{lrrr}\mathrm{C} & -0.988545 & -1.269802 & -0.468658 \\ \mathrm{C} & -0.134137 & -1.273546 & 0.810942 \\ \mathrm{C} & 0.743569 & -0.000000 & 0.904998 \\ \mathrm{C} & -0.134137 & 1.273546 & 0.810942 \\ \mathrm{C} & -0.988545 & 1.269802 & -0.468658 \\ \mathrm{C} & -1.856516 & 0.000000 & -0.550015 \\ \mathrm{H} & -0.789955 & -1.288625 & 1.691629 \\ \mathrm{H} & 0.497177 & -2.167249 & 0.853344 \\ \mathrm{H} & -0.329609 & -1.318951 & -1.346439 \\ \mathrm{H} & -1.619867 & -2.164881 & -0.491559 \\ \mathrm{H} & -0.789955 & 1.288625 & 1.691629 \\ \mathrm{H} & 0.497177 & 2.167249 & 0.853344 \\ \mathrm{H} & -1.619867 & 2.164881 & -0.491559 \\ \mathrm{H} & -0.329609 & 1.318951 & -1.346439 \\ \mathrm{H} & -2.577231 & 0.000000 & 0.280683 \\ \mathrm{H} & -2.436945 & 0.000000 & -1.479184 \\ \mathrm{C} & 1.753868 & -0.000000 & -0.173826 \\ \mathrm{~N} & 2.548276 & 0.000000 & -1.038048 \\ \mathrm{H} & 1.287406 & -0.000000 & 1.856542\end{array}$

Frequencies (Top 10 out of 51)

1. $\quad 114.1597 \mathrm{~cm}-1$ (Symmetry: A)

2. $\quad 152.7581 \mathrm{~cm}-1$ (Symmetry: A)

3. $258.6130 \mathrm{~cm}-1$ (Symmetry: A)

4. $264.2284 \mathrm{~cm}-1$ (Symmetry: A) 
5. $\quad 381.5719 \mathrm{~cm}-1$ (Symmetry: A)

6. $\quad 398.0374 \mathrm{~cm}-1$ (Symmetry: A)

7. $\quad 428.2321 \mathrm{~cm}-1$ (Symmetry: A)

8. $\quad 507.2265 \mathrm{~cm}-1$ (Symmetry: A)

9. $\quad 601.7199 \mathrm{~cm}-1$ (Symmetry: A)

10. $658.4291 \mathrm{~cm}-1$ (Symmetry: A)

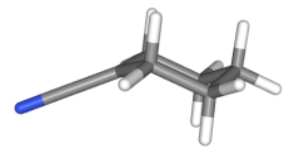

Charge

Multiplicity

Stoichiometry

Number of Basis Functions

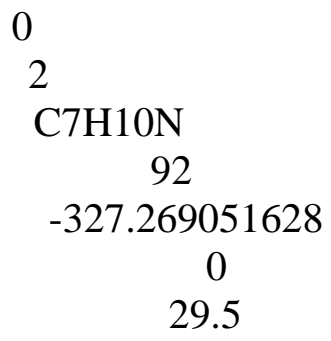

Electronic Energy (Eh)

Number of Imaginary Frequencies

Mean of alpha and beta Electrons

29.5

Molecular Geometry in Cartesian Coordinates

$\begin{array}{lrrr}\mathrm{C} & -0.391204 & -1.323943 & 1.269281 \\ \mathrm{C} & -0.391204 & 0.223895 & 1.291243 \\ \mathrm{C} & 0.171751 & 0.766664 & 0.000000 \\ \mathrm{C} & -0.391204 & 0.223895 & -1.291243 \\ \mathrm{C} & -0.391204 & -1.323943 & -1.269281 \\ \mathrm{C} & -1.080773 & -1.856164 & 0.000000 \\ \mathrm{H} & -1.432085 & 0.569197 & 1.396466 \\ \mathrm{H} & 0.167022 & 0.603501 & 2.151138 \\ \mathrm{H} & 0.645049 & -1.684773 & 1.298471 \\ \mathrm{H} & -0.894199 & -1.699665 & 2.166936 \\ \mathrm{H} & -1.432085 & 0.569197 & -1.396466 \\ \mathrm{H} & 0.167022 & 0.603501 & -2.151138 \\ \mathrm{H} & -0.894199 & -1.699665 & -2.166936 \\ \mathrm{H} & 0.645049 & -1.684773 & -1.298471 \\ \mathrm{H} & -2.134883 & -1.542871 & 0.000000 \\ \mathrm{H} & -1.071393 & -2.951697 & -0.000000 \\ \mathrm{C} & 1.165866 & 1.754670 & -0.000000 \\ \mathrm{~N} & 2.011791 & 2.589657 & -0.000000\end{array}$

Frequencies (Top 10 out of 48)

1. $\quad 50.8477 \mathrm{~cm}-1$ (Symmetry: A')

2. $\quad 149.3444$ cm-1 (Symmetry: A") 
3. $225.8461 \mathrm{~cm}-1$ (Symmetry: A")

4. $256.0897 \mathrm{~cm}-1$ (Symmetry: A')

5. $\quad 363.3859 \mathrm{~cm}-1$ (Symmetry: A')

6. $\quad 383.4507 \mathrm{~cm}-1$ (Symmetry: A')

7. $\quad 432.2770 \mathrm{~cm}-1$ (Symmetry: A")

8. $487.1994 \mathrm{~cm}-1$ (Symmetry: A')

9. $\quad 564.0310 \mathrm{~cm}-1$ (Symmetry: A')

10. 594.3617 cm-1 (Symmetry: A")

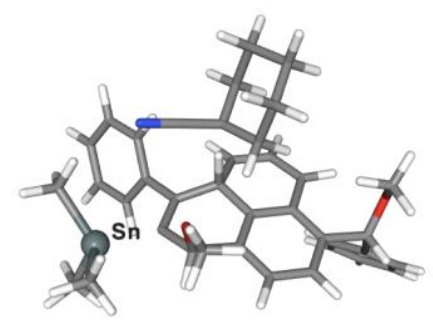

Charge

0

Multiplicity

Stoichiometry

Number of Basis Functions

Electronic Energy (Eh)

Number of Imaginary Frequencies

Mean of alpha and beta Electrons

3

$\mathrm{C} 40 \mathrm{H} 43 \mathrm{NO} 2 \mathrm{Sn}$

481

$-1757.56446037$

1

155

Molecular Geometry in Cartesian Coordinates

$\begin{array}{lccc}\mathrm{C} & -2.191461 & 1.240964 & -2.748285 \\ \mathrm{C} & -0.851287 & 0.874557 & -2.887810 \\ \mathrm{C} & -2.814741 & 1.209267 & -1.491505 \\ \mathrm{C} & -0.102070 & 0.486286 & -1.756283 \\ \mathrm{C} & -0.710339 & 0.436563 & -0.498213 \\ \mathrm{C} & -2.081399 & 0.807576 & -0.343804 \\ \mathrm{C} & -2.693045 & 0.728380 & 0.963566 \\ \mathrm{H} & -3.700158 & 1.101272 & 1.102048 \\ \mathrm{C} & -2.006758 & 0.079055 & 2.038923 \\ \mathrm{C} & -0.701390 & -0.312591 & 1.924449 \\ \mathrm{C} & 0.102359 & 0.033310 & 0.700863 \\ \mathrm{H} & -2.548874 & -0.112635 & 2.959845 \\ \mathrm{H} & -0.204055 & -0.837379 & 2.732808 \\ \mathrm{H} & 0.632638 & 1.171599 & 1.043194 \\ \mathrm{C} & 1.368535 & 0.173476 & -1.894121 \\ \mathrm{H} & 1.554552 & -0.240219 & -2.899995 \\ \mathrm{C} & 2.145184 & 2.263502 & -2.944478 \\ \mathrm{H} & 1.161251 & 2.733557 & -3.076716 \\ \mathrm{H} & 2.899351 & 3.033496 & -2.773993\end{array}$




\begin{tabular}{|c|c|c|c|}
\hline $\mathrm{H}$ & 2.400701 & 1.695143 & -3.850608 \\
\hline $\mathrm{O}$ & 2.174401 & 1.406178 & -1.778229 \\
\hline $\mathrm{C}$ & 1.926428 & -0.736116 & -0.820696 \\
\hline $\mathrm{C}$ & 1.314310 & -0.786399 & 0.397033 \\
\hline $\mathrm{C}$ & 1.921073 & -1.594535 & 1.498400 \\
\hline 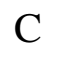 & 2.395138 & -0.983576 & 2.675326 \\
\hline$C$ & 2.067798 & -2.985488 & 1.347756 \\
\hline $\mathrm{C}$ & 3.019674 & -1.744282 & 3.671459 \\
\hline $\mathrm{H}$ & 2.310577 & 0.093906 & 2.791513 \\
\hline $\mathrm{C}$ & 2.692185 & -3.750797 & 2.345458 \\
\hline $\mathrm{H}$ & 1.685792 & -3.462475 & 0.449170 \\
\hline $\mathrm{C}$ & 3.172662 & -3.131855 & 3.508954 \\
\hline $\mathrm{H}$ & 3.399801 & -1.256598 & 4.563112 \\
\hline $\mathrm{H}$ & 2.800777 & -4.822729 & 2.213839 \\
\hline $\mathrm{H}$ & 3.660668 & -3.720144 & 4.279130 \\
\hline $\mathrm{Sn}$ & 3.896745 & -1.497759 & -1.195331 \\
\hline $\mathrm{C}$ & 3.826736 & -3.591284 & -1.631075 \\
\hline $\mathrm{H}$ & 2.882797 & -3.860887 & -2.119026 \\
\hline $\mathrm{H}$ & 4.650368 & -3.870071 & -2.298971 \\
\hline $\mathrm{H}$ & 3.919796 & -4.174032 & -0.707374 \\
\hline $\mathrm{C}$ & 4.562376 & -0.486040 & -2.950913 \\
\hline $\mathrm{H}$ & 3.868401 & -0.638092 & -3.787535 \\
\hline $\mathrm{H}$ & 4.630563 & 0.587130 & -2.744160 \\
\hline $\mathrm{H}$ & 5.548473 & -0.851612 & -3.260096 \\
\hline $\mathrm{C}$ & 5.176436 & -1.136179 & 0.457066 \\
\hline $\mathrm{H}$ & 6.225706 & -1.265957 & 0.166552 \\
\hline $\mathrm{H}$ & 5.034128 & -0.113025 & 0.822139 \\
\hline $\mathrm{H}$ & 4.950655 & -1.834076 & 1.272683 \\
\hline $\mathrm{H}$ & -0.379030 & 0.884039 & -3.866587 \\
\hline $\mathrm{H}$ & -2.765875 & 1.542562 & -3.620419 \\
\hline $\mathrm{C}$ & -4.288789 & 1.585816 & -1.397366 \\
\hline $\mathrm{H}$ & -4.619305 & 1.910188 & -2.396964 \\
\hline $\mathrm{C}$ & -3.843170 & 3.900772 & -0.825119 \\
\hline $\mathrm{H}$ & -2.753833 & 3.780794 & -0.761838 \\
\hline $\mathrm{H}$ & -4.109752 & 4.209743 & -1.845909 \\
\hline $\mathrm{H}$ & -4.174401 & 4.658334 & -0.115018 \\
\hline $\mathrm{C}$ & -5.123309 & 0.450020 & -0.973999 \\
\hline $\mathrm{C}$ & -5.792819 & -0.509249 & -0.635015 \\
\hline $\mathrm{C}$ & -6.542344 & -1.659007 & -0.201142 \\
\hline $\mathrm{C}$ & -5.906416 & -2.665460 & 0.555921 \\
\hline $\mathrm{C}$ & -7.908749 & -1.793141 & -0.521217 \\
\hline $\mathrm{C}$ & -6.627807 & -3.786370 & 0.984137 \\
\hline $\mathrm{H}$ & -4.853871 & -2.557270 & 0.798600 \\
\hline $\mathrm{C}$ & -8.626029 & -2.916087 & -0.090685 \\
\hline $\mathrm{H}$ & -8.395409 & -1.016203 & -1.101480 \\
\hline $\mathrm{C}$ & -7.988683 & -3.915248 & 0.662390 \\
\hline $\mathrm{H}$ & -6.132385 & -4.556884 & 1.565522 \\
\hline
\end{tabular}




$\begin{array}{lccc}\mathrm{H} & -9.677537 & -3.012943 & -0.339868 \\ \mathrm{H} & -8.546245 & -4.784663 & 0.994869 \\ \mathrm{O} & -4.529903 & 2.679139 & -0.460484 \\ \mathrm{C} & 1.122979 & 2.484384 & 1.368605 \\ \mathrm{C} & 0.475971 & 3.381996 & 0.320139 \\ \mathrm{C} & 0.608372 & 2.743911 & 2.783581 \\ \mathrm{C} & 0.697464 & 4.866833 & 0.696023 \\ \mathrm{H} & -0.603821 & 3.170265 & 0.310732 \\ \mathrm{H} & 0.876264 & 3.154285 & -0.671361 \\ \mathrm{C} & 0.834927 & 4.229801 & 3.154122 \\ \mathrm{H} & -0.468261 & 2.525186 & 2.802172 \\ \mathrm{H} & 1.094944 & 2.084867 & 3.511395 \\ \mathrm{C} & 0.174900 & 5.155987 & 2.115650 \\ \mathrm{H} & 0.192955 & 5.508164 & -0.036445 \\ \mathrm{H} & 1.771036 & 5.094334 & 0.644854 \\ \mathrm{H} & 0.429119 & 4.423163 & 4.153679 \\ \mathrm{H} & 1.914259 & 4.430707 & 3.193592 \\ \mathrm{H} & 0.357983 & 6.204190 & 2.378129 \\ \mathrm{H} & -0.914109 & 5.002214 & 2.136942 \\ \mathrm{C} & 2.543259 & 2.292694 & 1.250315 \\ \mathrm{~N} & 3.701593 & 2.077928 & 1.173381\end{array}$

Frequencies (Top 10 out of 255)

1. $-1517.5962 \mathrm{~cm}-1$ (Symmetry: A) *

2. $\quad 11.0157 \mathrm{~cm}-1$ (Symmetry: A)

3. $\quad 13.5432 \mathrm{~cm}-1$ (Symmetry: A)

4. $\quad 16.9489 \mathrm{~cm}-1$ (Symmetry: A)

5. $21.9229 \mathrm{~cm}-1$ (Symmetry: A)

6. $\quad 28.2512 \mathrm{~cm}-1$ (Symmetry: A)

7. $\quad 37.1703 \mathrm{~cm}-1$ (Symmetry: A)

8. $\quad 45.9388 \mathrm{~cm}-1$ (Symmetry: A)

9. $\quad 51.6478 \mathrm{~cm}-1$ (Symmetry: A)

10. $\quad 56.4882 \mathrm{~cm}-1$ (Symmetry: A)

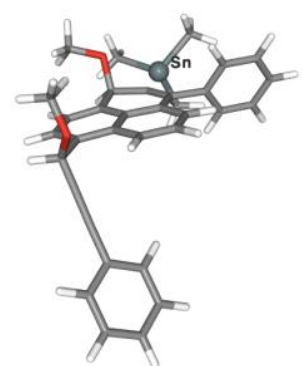

Charge 
Multiplicity

Stoichiometry

Number of Basis Functions

Electronic Energy (Eh)

Number of Imaginary Frequencies

Mean of alpha and beta Electrons
1

C33H32O2Sn

387

$-1429.76202574$

0

125

Molecular Geometry in Cartesian Coordinates

$\begin{array}{lrrr}\mathrm{C} & -2.073696 & 2.879733 & -0.614202 \\ \mathrm{C} & -0.705335 & 2.723118 & -0.958741 \\ \mathrm{C} & -2.686913 & 2.010475 & 0.268349 \\ \mathrm{C} & 0.051901 & 1.706391 & -0.403029 \\ \mathrm{C} & -0.569396 & 0.758933 & 0.468329 \\ \mathrm{C} & -1.943168 & 0.918320 & 0.828157 \\ \mathrm{C} & -2.544208 & -0.031221 & 1.713987 \\ \mathrm{H} & -3.572526 & 0.109940 & 2.024574 \\ \mathrm{C} & -1.812662 & -1.111426 & 2.166911 \\ \mathrm{C} & -0.464791 & -1.302164 & 1.758499 \\ \mathrm{C} & 0.168909 & -0.384246 & 0.930860 \\ \mathrm{H} & -2.272570 & -1.835437 & 2.831131 \\ \mathrm{H} & 0.067145 & -2.187406 & 2.089926 \\ \mathrm{C} & 1.537239 & 1.625992 & -0.680442 \\ \mathrm{H} & 1.721465 & 1.931011 & -1.725158 \\ \mathrm{C} & 2.127982 & 3.960877 & -0.121283 \\ \mathrm{H} & 1.142489 & 4.325743 & 0.190591 \\ \mathrm{H} & 2.902744 & 4.484284 & 0.441034 \\ \mathrm{H} & 2.265121 & 4.150723 & -1.196781 \\ \mathrm{O} & 2.293926 & 2.556275 & 0.179830 \\ \mathrm{C} & 2.186138 & 0.292860 & -0.370105 \\ \mathrm{C} & 1.557214 & -0.594338 & 0.444564 \\ \mathrm{C} & 2.285327 & -1.822453 & 0.896378 \\ \mathrm{C} & 2.670110 & -1.963214 & 2.244493 \\ \mathrm{C} & 2.648928 & -2.820883 & -0.024045 \\ \mathrm{C} & 3.416410 & -3.073568 & 2.659761 \\ \mathrm{H} & 2.401167 & -1.186942 & 2.955607 \\ \mathrm{C} & 3.398530 & -3.934572 & 0.389482 \\ \mathrm{H} & 2.340136 & -2.722674 & -1.061270 \\ \mathrm{C} & 3.786027 & -4.062484 & 1.731491 \\ \mathrm{H} & 3.717753 & -3.163788 & 3.698356 \\ \mathrm{H} & 3.674899 & -4.696538 & -0.332448 \\ \mathrm{H} & 4.367212 & -4.920890 & 2.052280 \\ \mathrm{Sn} & 4.246943 & 0.155911 & -0.939145 \\ \mathrm{C} & 4.525911 & -1.294191 & -2.486412 \\ \mathrm{H} & 3.632354 & -1.374555 & -3.116330 \\ \mathrm{H} & 5.370883 & -1.010041 & -3.124773\end{array}$




$\begin{array}{lrrr}\mathrm{H} & 4.734936 & -2.278766 & -2.053032 \\ \mathrm{C} & 4.789531 & 2.055020 & -1.741530 \\ \mathrm{H} & 4.065467 & 2.385345 & -2.497305 \\ \mathrm{H} & 4.811536 & 2.800418 & -0.940248 \\ \mathrm{H} & 5.776550 & 2.009680 & -2.216376 \\ \mathrm{C} & 5.445769 & -0.354251 & 0.740356 \\ \mathrm{H} & 6.502661 & -0.138415 & 0.546207 \\ \mathrm{H} & 5.126997 & 0.214956 & 1.620274 \\ \mathrm{H} & 5.340763 & -1.422569 & 0.965619 \\ \mathrm{H} & -0.249500 & 3.414081 & -1.663147 \\ \mathrm{H} & -2.645748 & 3.692215 & -1.054457 \\ \mathrm{C} & -4.160826 & 2.220680 & 0.603113 \\ \mathrm{H} & -4.483474 & 3.154182 & 0.114842 \\ \mathrm{C} & -3.678599 & 3.448155 & 2.639484 \\ \mathrm{H} & -2.596382 & 3.271770 & 2.623710 \\ \mathrm{H} & -3.898511 & 4.391169 & 2.118923 \\ \mathrm{H} & -4.028344 & 3.507802 & 3.669792 \\ \mathrm{C} & -5.003288 & 1.120357 & 0.107299 \\ \mathrm{C} & -5.687542 & 0.210678 & -0.325474 \\ \mathrm{C} & -6.464636 & -0.892050 & -0.827954 \\ \mathrm{C} & -5.866166 & -2.159116 & -0.990174 \\ \mathrm{C} & -7.824121 & -0.721455 & -1.160488 \\ \mathrm{C} & -6.617268 & -3.235578 & -1.477626 \\ \mathrm{H} & -4.819406 & -2.285159 & -0.732393 \\ \mathrm{C} & -8.571223 & -1.801022 & -1.647512 \\ \mathrm{H} & -8.282192 & 0.253611 & -1.031658 \\ \mathrm{C} & -7.970874 & -3.060162 & -1.807755 \\ \mathrm{H} & -6.150489 & -4.207315 & -1.600076 \\ \mathrm{H} & -9.617216 & -1.663139 & -1.900598 \\ \mathrm{H} & -8.551516 & -3.895438 & -2.185247 \\ \mathrm{O} & -4.400031 & 2.345900 & 2.034828\end{array}$

Frequencies (Top 10 out of 198)

1. $\quad 11.3280 \mathrm{~cm}-1$ (Symmetry: A)

2. $\quad 13.0645 \mathrm{~cm}-1$ (Symmetry: A)

3. $\quad 15.9661 \mathrm{~cm}-1$ (Symmetry: A)

4. $\quad 27.6838 \mathrm{~cm}-1$ (Symmetry: A)

5. $\quad 35.0916 \mathrm{~cm}-1$ (Symmetry: A)

6. $\quad 43.1658 \mathrm{~cm}-1$ (Symmetry: A)

7. $\quad 51.0116 \mathrm{~cm}-1$ (Symmetry: A)

8. $\quad 56.1183 \mathrm{~cm}-1$ (Symmetry: A)

9. $\quad 65.7518 \mathrm{~cm}-1$ (Symmetry: A)

10. $\quad 69.4279 \mathrm{~cm}-1$ (Symmetry: A) 


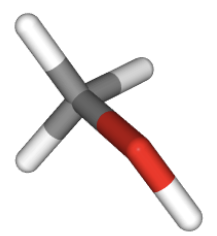

Charge

Multiplicity

Stoichiometry

Number of Basis Functions

Electronic Energy (Eh)

Number of Imaginary Frequencies

Mean of alpha and beta Electrons
0

1

$\mathrm{CH} 4 \mathrm{O}$

26

$-115.650629547$

0

9

Molecular Geometry in Cartesian Coordinates

$\begin{array}{lccc}\mathrm{O} & -0.757985 & 0.122330 & 0.000002 \\ \mathrm{C} & 0.683476 & -0.022349 & 0.000006 \\ \mathrm{H} & 1.042857 & -0.545080 & 0.895352 \\ \mathrm{H} & 1.042670 & -0.547871 & -0.893786 \\ \mathrm{H} & 1.087492 & 0.989648 & -0.001643 \\ \mathrm{H} & -1.209996 & -0.741245 & 0.000022\end{array}$

Frequencies (Top 10 out of 12)

1. $\quad 358.0770 \mathrm{~cm}-1$ (Symmetry: A)

2. $1042.6312 \mathrm{~cm}-1$ (Symmetry: A)

3. $1084.9272 \mathrm{~cm}-1$ (Symmetry: A)

4. $\quad 1162.7450 \mathrm{~cm}-1$ (Symmetry: A)

5. $1347.8695 \mathrm{~cm}-1$ (Symmetry: A)

6. $\quad 1507.0028 \mathrm{~cm}-1$ (Symmetry: A)

7. $1527.8308 \mathrm{~cm}-1$ (Symmetry: A)

8. $\quad 1534.7022 \mathrm{~cm}-1$ (Symmetry: A)

9. $3053.0515 \mathrm{~cm}-1$ (Symmetry: A)

10. $3129.8651 \mathrm{~cm}-1$ (Symmetry: A) 


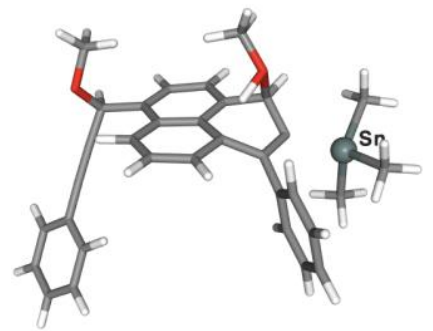

Charge

Multiplicity

Stoichiometry

Number of Basis Functions

0

Electronic Energy (Eh)

2

Number of Imaginary Frequencies

$\mathrm{C} 33 \mathrm{H} 33 \mathrm{O} 2 \mathrm{Sn}$

389

Mean of alpha and beta Electrons

$-1430.24921378$

1

125.5

Molecular Geometry in Cartesian Coordinates

$\begin{array}{lrrr}\mathrm{C} & -1.845591 & 2.855050 & -1.353608 \\ \mathrm{C} & -0.445847 & 2.776561 & -1.450608 \\ \mathrm{C} & -2.546910 & 2.189868 & -0.338990 \\ \mathrm{C} & 0.242804 & 1.967298 & -0.534941 \\ \mathrm{C} & -0.442257 & 1.249625 & 0.465864 \\ \mathrm{C} & -1.851591 & 1.397112 & 0.629982 \\ \mathrm{C} & -2.480985 & 0.763494 & 1.747994 \\ \mathrm{H} & -3.543616 & 0.900124 & 1.909900 \\ \mathrm{C} & -1.711792 & -0.016139 & 2.641917 \\ \mathrm{C} & -0.348330 & -0.217495 & 2.471093 \\ \mathrm{C} & 0.374276 & 0.428880 & 1.386267 \\ \mathrm{H} & -2.213895 & -0.492022 & 3.479098 \\ \mathrm{H} & 0.185926 & -0.881249 & 3.143419 \\ \mathrm{H} & 1.273335 & 1.589724 & 1.653267 \\ \mathrm{C} & 1.724938 & 1.901110 & -0.362545 \\ \mathrm{H} & 2.305266 & 2.575557 & -0.994785 \\ \mathrm{C} & 1.401423 & 3.814119 & 1.380336 \\ \mathrm{H} & 0.308100 & 3.781840 & 1.443031 \\ \mathrm{H} & 1.832960 & 4.159601 & 2.320115 \\ \mathrm{H} & 1.702079 & 4.471462 & 0.559891 \\ \mathrm{O} & 1.945634 & 2.484186 & 1.130455 \\ \mathrm{C} & 2.248286 & 0.505738 & -0.217407 \\ \mathrm{C} & 1.569141 & -0.197605 & 0.752569 \\ \mathrm{C} & 2.054400 & -1.530252 & 1.207079 \\ \mathrm{C} & 2.623423 & -1.699908 & 2.483355 \\ \mathrm{C} & 1.999768 & -2.629484 & 0.330103 \\ \mathrm{C} & 3.139985 & -2.943173 & 2.871732 \\ \mathrm{H} & 2.686698 & -0.847921 & 3.155432\end{array}$




\begin{tabular}{|c|c|c|c|}
\hline $\mathrm{C}$ & 2.517843 & -3.874864 & 0.717794 \\
\hline $\mathrm{H}$ & 1.543731 & -2.504261 & -0.648518 \\
\hline $\mathrm{C}$ & 3.092274 & -4.034369 & 1.988462 \\
\hline $\mathrm{H}$ & 3.586872 & -3.059634 & 3.853903 \\
\hline $\mathrm{H}$ & 2.470462 & -4.715416 & 0.032725 \\
\hline $\mathrm{H}$ & 3.495415 & -4.996051 & 2.288532 \\
\hline $\mathrm{Sn}$ & 4.023789 & -0.149559 & -1.175777 \\
\hline $\mathrm{C}$ & 3.594377 & -1.542771 & -2.733134 \\
\hline $\mathrm{H}$ & 2.607240 & -1.344452 & -3.165405 \\
\hline $\mathrm{H}$ & 4.341583 & -1.475773 & -3.532337 \\
\hline $\mathrm{H}$ & 3.601231 & -2.565771 & -2.340047 \\
\hline $\mathrm{C}$ & 4.862289 & 1.619691 & -2.029441 \\
\hline $\mathrm{H}$ & 4.207335 & 2.030598 & -2.807438 \\
\hline $\mathrm{H}$ & 5.016978 & 2.388493 & -1.262916 \\
\hline $\mathrm{H}$ & 5.832698 & 1.402502 & -2.490235 \\
\hline $\mathrm{C}$ & 5.359497 & -1.027320 & 0.227595 \\
\hline $\mathrm{H}$ & 6.348848 & -1.182509 & -0.218193 \\
\hline $\mathrm{H}$ & 5.469149 & -0.384687 & 1.108152 \\
\hline $\mathrm{H}$ & 4.968566 & -1.996694 & 0.560992 \\
\hline $\mathrm{H}$ & 0.090938 & 3.333820 & -2.212509 \\
\hline $\mathrm{H}$ & -2.399878 & 3.458388 & -2.068356 \\
\hline $\mathrm{C}$ & -4.061477 & 2.327847 & -0.286107 \\
\hline $\mathrm{H}$ & -4.370010 & 3.001346 & -1.101842 \\
\hline $\mathrm{C}$ & -3.960275 & 4.181670 & 1.279098 \\
\hline $\mathrm{H}$ & -2.886986 & 4.094280 & 1.490975 \\
\hline $\mathrm{H}$ & -4.104886 & 4.886134 & 0.446848 \\
\hline $\mathrm{H}$ & -4.483289 & 4.546178 & 2.163452 \\
\hline $\mathrm{C}$ & -4.736884 & 1.031783 & -0.464153 \\
\hline $\mathrm{C}$ & -5.263773 & -0.054235 & -0.626177 \\
\hline $\mathrm{C}$ & -5.838570 & -1.364751 & -0.778770 \\
\hline $\mathrm{C}$ & -5.188450 & -2.482485 & -0.214317 \\
\hline $\mathrm{C}$ & -7.044657 & -1.550697 & -1.484171 \\
\hline $\mathrm{C}$ & -5.738270 & -3.762581 & -0.354218 \\
\hline $\mathrm{H}$ & -4.258647 & -2.333029 & 0.325684 \\
\hline $\mathrm{C}$ & -7.590010 & -2.833176 & -1.622781 \\
\hline $\mathrm{H}$ & -7.543725 & -0.689185 & -1.915484 \\
\hline $\mathrm{C}$ & -6.939598 & -3.942303 & -1.058742 \\
\hline $\mathrm{H}$ & -5.232728 & -4.617021 & 0.083637 \\
\hline $\mathrm{H}$ & -8.519233 & -2.968642 & -2.166439 \\
\hline $\mathrm{H}$ & -7.364305 & -4.935058 & -1.167102 \\
\hline $\mathrm{O}$ & -4.540684 & 2.892045 & 0.973870 \\
\hline
\end{tabular}

Frequencies (Top 10 out of 201)

1. $-1626.3993 \mathrm{~cm}-1$ (Symmetry: A) * 
2. $\quad 13.2232 \mathrm{~cm}-1$ (Symmetry: A)

3. $\quad 16.1155 \mathrm{~cm}-1$ (Symmetry: A)

4. $\quad 26.4270 \mathrm{~cm}-1$ (Symmetry: A)

5. $\quad 31.3396 \mathrm{~cm}-1$ (Symmetry: A)

6. $\quad 36.0095 \mathrm{~cm}-1$ (Symmetry: A)

7. $\quad 43.5730 \mathrm{~cm}-1$ (Symmetry: A)

8. $\quad 54.5968 \mathrm{~cm}-1$ (Symmetry: A)

9. $\quad 62.0786 \mathrm{~cm}-1$ (Symmetry: A)

10. $\quad 65.4886 \mathrm{~cm}-1$ (Symmetry: A)

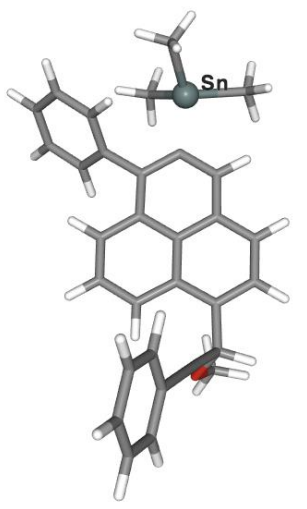

Charge

Multiplicity

Stoichiometry

Number of Basis Functions

0

Electronic Energy (Eh)

Number of Imaginary Frequencies

Mean of alpha and beta Electrons

2

$\mathrm{C} 32 \mathrm{H} 29 \mathrm{OSn}$

363

$-1314.69765336$

0

116.5

Molecular Geometry in Cartesian Coordinates

$\begin{array}{lrrc}\mathrm{C} & 1.964143 & -2.967727 & -0.352640 \\ \mathrm{C} & 0.631680 & -2.873435 & -0.763565 \\ \mathrm{C} & 2.563424 & -1.963425 & 0.416018 \\ \mathrm{C} & -0.155035 & -1.748994 & -0.396836 \\ \mathrm{C} & 0.431903 & -0.714558 & 0.399640 \\ \mathrm{C} & 1.803568 & -0.814065 & 0.810224 \\ \mathrm{C} & 2.363876 & 0.232470 & 1.596197 \\ \mathrm{H} & 3.389590 & 0.153873 & 1.936003 \\ \mathrm{C} & 1.593457 & 1.350452 & 1.932556 \\ \mathrm{C} & 0.257612 & 1.457237 & 1.532797 \\ \mathrm{C} & -0.361891 & 0.419879 & 0.777845 \\ \mathrm{H} & 2.042458 & 2.149698 & 2.512915 \\ \mathrm{H} & -0.313457 & 2.342129 & 1.791413 \\ \mathrm{C} & -1.517639 & -1.632998 & -0.789877 \\ \mathrm{H} & -1.941094 & -2.444681 & -1.380471\end{array}$




\begin{tabular}{|c|c|c|c|}
\hline $\mathrm{C}$ & -2.316510 & -0.539130 & -0.417225 \\
\hline $\mathrm{C}$ & -1.738745 & 0.486999 & 0.370056 \\
\hline $\mathrm{C}$ & -2.592546 & 1.633098 & 0.800934 \\
\hline $\mathrm{C}$ & -2.877841 & 1.849487 & 2.164004 \\
\hline $\mathrm{C}$ & -3.177042 & 2.483227 & -0.157141 \\
\hline $\mathrm{C}$ & -3.742838 & 2.878979 & 2.557609 \\
\hline $\mathrm{H}$ & -2.436052 & 1.191638 & 2.907479 \\
\hline $\mathrm{C}$ & -4.042290 & 3.516622 & 0.234555 \\
\hline $\mathrm{H}$ & -2.938952 & 2.339787 & -1.208090 \\
\hline $\mathrm{C}$ & -4.331640 & 3.714391 & 1.593167 \\
\hline $\mathrm{H}$ & -3.963133 & 3.025879 & 3.610004 \\
\hline $\mathrm{H}$ & -4.482041 & 4.165714 & -0.516178 \\
\hline $\mathrm{H}$ & -5.002576 & 4.510737 & 1.898233 \\
\hline $\mathrm{Sn}$ & -4.402779 & -0.605679 & -0.913920 \\
\hline $\mathrm{C}$ & -4.907535 & 0.675506 & -2.543898 \\
\hline $\mathrm{H}$ & -4.041811 & 0.843181 & -3.194537 \\
\hline $\mathrm{H}$ & -5.705852 & 0.225666 & -3.145401 \\
\hline $\mathrm{H}$ & -5.258240 & 1.644951 & -2.172734 \\
\hline $\mathrm{C}$ & -4.741702 & -2.622548 & -1.522730 \\
\hline $\mathrm{H}$ & -4.223304 & -2.843516 & -2.463314 \\
\hline $\mathrm{H}$ & -4.390998 & -3.328752 & -0.761359 \\
\hline $\mathrm{H}$ & -5.812324 & -2.796889 & -1.680903 \\
\hline $\mathrm{C}$ & -5.571504 & -0.125207 & 0.796192 \\
\hline $\mathrm{H}$ & -6.612183 & -0.440980 & 0.659708 \\
\hline $\mathrm{H}$ & -5.171892 & -0.620780 & 1.687901 \\
\hline $\mathrm{H}$ & -5.553236 & 0.957000 & 0.972628 \\
\hline $\mathrm{H}$ & 0.183169 & -3.660009 & -1.363175 \\
\hline $\mathrm{H}$ & 2.552652 & -3.836220 & -0.638090 \\
\hline $\mathrm{C}$ & 4.020894 & -2.130097 & 0.823321 \\
\hline $\mathrm{H}$ & 4.352685 & -3.119461 & 0.468188 \\
\hline $\mathrm{C}$ & 3.460306 & -3.090560 & 2.980294 \\
\hline $\mathrm{H}$ & 2.381516 & -2.904824 & 2.912383 \\
\hline $\mathrm{H}$ & 3.678620 & -4.092140 & 2.582432 \\
\hline $\mathrm{H}$ & 3.781917 & -3.031017 & 4.019937 \\
\hline $\mathrm{C}$ & 4.903807 & -1.110517 & 0.231489 \\
\hline $\mathrm{C}$ & 5.653720 & -0.293235 & -0.271677 \\
\hline $\mathrm{C}$ & 6.550937 & 0.673936 & -0.848500 \\
\hline $\mathrm{C}$ & 6.143171 & 1.476188 & -1.934041 \\
\hline $\mathrm{C}$ & 7.852852 & 0.826112 & -0.327472 \\
\hline $\mathrm{C}$ & 7.025077 & 2.412512 & -2.488151 \\
\hline $\mathrm{H}$ & 5.140468 & 1.359335 & -2.331704 \\
\hline $\mathrm{C}$ & 8.729861 & 1.765184 & -0.883164 \\
\hline $\mathrm{H}$ & 8.161690 & 0.208778 & 0.509603 \\
\hline $\mathrm{C}$ & 8.319787 & 2.560481 & -1.965332 \\
\hline $\mathrm{H}$ & 6.704950 & 3.025762 & -3.324090 \\
\hline $\mathrm{H}$ & 9.728916 & 1.877696 & -0.475098 \\
\hline $\mathrm{H}$ & 9.001160 & 3.287261 & -2.395455 \\
\hline
\end{tabular}


$\begin{array}{llll}\text { O } & 4.214299 & -2.077930 & 2.268738\end{array}$

Frequencies (Top 10 out of 183)

1. $\quad 10.7575 \mathrm{~cm}-1$ (Symmetry: A)

2. $\quad 15.8860 \mathrm{~cm}-1$ (Symmetry: A)

3. $20.8455 \mathrm{~cm}-1$ (Symmetry: A)

4. $\quad 36.0329 \mathrm{~cm}-1$ (Symmetry: A)

5. $39.1193 \mathrm{~cm}-1$ (Symmetry: A)

6. $\quad 47.7017 \mathrm{~cm}-1$ (Symmetry: A)

7. $\quad 56.8245 \mathrm{~cm}-1$ (Symmetry: A)

8. $\quad 63.4051 \mathrm{~cm}-1$ (Symmetry: A)

9. $\quad 69.8807 \mathrm{~cm}-1$ (Symmetry: A)

10. $\quad 76.1681 \mathrm{~cm}-1$ (Symmetry: A)

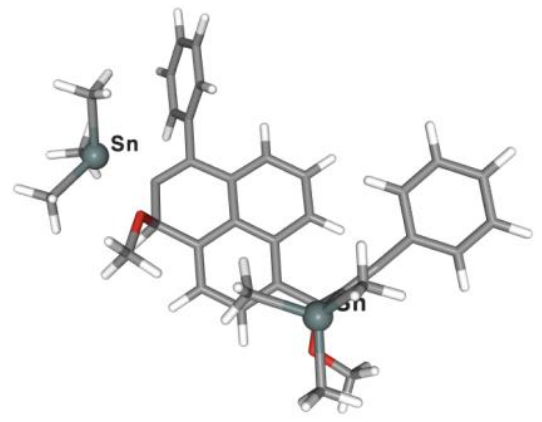

Charge

Multiplicity

Stoichiometry

Number of Basis Functions

Electronic Energy (Eh)

Number of Imaginary Frequencies

Mean of alpha and beta Electrons

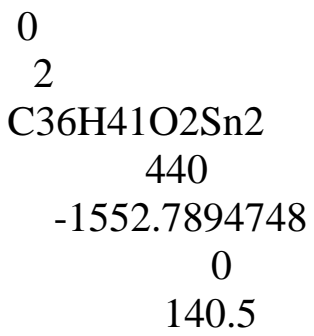

Molecular Geometry in Cartesian Coordinates

$\begin{array}{lrrr}\mathrm{C} & -1.216014 & -2.101536 & 1.646338 \\ \mathrm{C} & 0.127680 & -2.339511 & 1.254294 \\ \mathrm{C} & -1.720374 & -0.814389 & 1.681389 \\ \mathrm{C} & 0.958427 & -1.297794 & 0.879961 \\ \mathrm{C} & 0.492187 & 0.047506 & 0.996285 \\ \mathrm{C} & -0.857678 & 0.297531 & 1.395410 \\ \mathrm{C} & -1.292517 & 1.654144 & 1.527675 \\ \mathrm{H} & -2.310738 & 1.872605 & 1.832085 \\ \mathrm{C} & -0.415985 & 2.696111 & 1.296709 \\ \mathrm{C} & 0.933750 & 2.442848 & 0.934371\end{array}$




\begin{tabular}{|c|c|c|c|}
\hline $\mathrm{C}$ & 1.397649 & 1.142866 & 0.778220 \\
\hline $\mathrm{H}$ & -0.752193 & 3.720944 & 1.415840 \\
\hline H & 1.612412 & 3.278817 & 0.805036 \\
\hline $\mathrm{C}$ & 2.331950 & -1.580426 & 0.315505 \\
\hline $\mathrm{H}$ & 2.754697 & -2.461320 & 0.828987 \\
\hline $\mathrm{C}$ & 1.653582 & -3.182716 & -1.439496 \\
\hline $\mathrm{H}$ & 0.568190 & -3.156045 & -1.287186 \\
\hline $\mathrm{H}$ & 1.866694 & -3.365305 & -2.494002 \\
\hline $\mathrm{H}$ & 2.090790 & -3.989909 & -0.832064 \\
\hline $\mathrm{O}$ & 2.264753 & -1.910384 & -1.121892 \\
\hline C & 3.296159 & -0.411954 & 0.332889 \\
\hline $\mathrm{C}$ & 2.823785 & 0.853996 & 0.475588 \\
\hline $\mathrm{C}$ & 3.754949 & 2.012712 & 0.293399 \\
\hline $\mathrm{C}$ & 3.620466 & 2.857950 & -0.825904 \\
\hline $\mathrm{C}$ & 4.809446 & 2.238724 & 1.194993 \\
\hline $\mathrm{C}$ & 4.531437 & 3.898947 & -1.046161 \\
\hline $\mathrm{H}$ & 2.813942 & 2.677244 & -1.531246 \\
\hline $\mathrm{C}$ & 5.725359 & 3.281154 & 0.975733 \\
\hline $\mathrm{H}$ & 4.906635 & 1.598092 & 2.067297 \\
\hline $\mathrm{C}$ & 5.590070 & 4.112091 & -0.146121 \\
\hline $\mathrm{H}$ & 4.424738 & 4.534417 & -1.919360 \\
\hline $\mathrm{H}$ & 6.536615 & 3.442430 & 1.678602 \\
\hline $\mathrm{H}$ & 6.298272 & 4.916080 & -0.318255 \\
\hline $\mathrm{Sn}$ & 5.282006 & -0.894774 & -0.309193 \\
\hline $\mathrm{C}$ & 6.689182 & -0.658109 & 1.284377 \\
\hline $\mathrm{H}$ & 6.216740 & -0.838897 & 2.256916 \\
\hline $\mathrm{H}$ & 7.516681 & -1.368019 & 1.168126 \\
\hline $\mathrm{H}$ & 7.101024 & 0.357418 & 1.281785 \\
\hline $\mathrm{C}$ & 5.272144 & -2.957318 & -0.847033 \\
\hline $\mathrm{H}$ & 4.842881 & -3.572069 & -0.045658 \\
\hline $\mathrm{H}$ & 4.667822 & -3.099280 & -1.748524 \\
\hline $\mathrm{H}$ & 6.290847 & -3.314510 & -1.038010 \\
\hline $\mathrm{C}$ & 5.848526 & 0.337393 & -1.946037 \\
\hline $\mathrm{H}$ & 6.719105 & -0.079003 & -2.465689 \\
\hline $\mathrm{H}$ & 5.021465 & 0.422667 & -2.659182 \\
\hline $\mathrm{H}$ & 6.100141 & 1.343472 & -1.588852 \\
\hline $\mathrm{H}$ & 0.499786 & -3.360974 & 1.229521 \\
\hline $\mathrm{H}$ & -1.867118 & -2.924716 & 1.914160 \\
\hline $\mathrm{C}$ & -3.191200 & -0.583278 & 1.984179 \\
\hline $\mathrm{H}$ & -3.313119 & 0.166271 & 2.781660 \\
\hline $\mathrm{C}$ & -5.103970 & -1.731935 & 2.926796 \\
\hline $\mathrm{H}$ & -5.141517 & -1.049720 & 3.786201 \\
\hline $\mathrm{H}$ & -5.786132 & -1.368576 & 2.147388 \\
\hline $\mathrm{H}$ & -5.401298 & -2.733751 & 3.237684 \\
\hline $\mathrm{C}$ & -3.926271 & -0.109324 & 0.715405 \\
\hline $\mathrm{C}$ & -4.378248 & 1.104810 & 0.528798 \\
\hline $\mathrm{C}$ & -4.878570 & 2.285793 & -0.024528 \\
\hline
\end{tabular}




$\begin{array}{lrrr}\text { C } & -6.246031 & 2.672041 & 0.143835 \\ \mathrm{C} & -4.012586 & 3.159163 & -0.759965 \\ \mathrm{C} & -6.716581 & 3.862503 & -0.407724 \\ \mathrm{H} & -6.909623 & 2.021859 & 0.705291 \\ \mathrm{C} & -4.503694 & 4.345179 & -1.302867 \\ \mathrm{H} & -2.971232 & 2.876470 & -0.886067 \\ \mathrm{C} & -5.854950 & 4.709079 & -1.133999 \\ \mathrm{H} & -7.757972 & 4.138793 & -0.276350 \\ \mathrm{H} & -3.836833 & 4.993251 & -1.862643 \\ \mathrm{H} & -6.228992 & 5.634066 & -1.559303 \\ \mathrm{O} & -3.751630 & -1.843252 & 2.425014 \\ \mathrm{Sn} & -4.149047 & -1.538083 & -0.896006 \\ \mathrm{C} & -2.243046 & -2.225952 & -1.557267 \\ \mathrm{H} & -1.965594 & -3.135212 & -1.012309 \\ \mathrm{H} & -2.265793 & -2.448278 & -2.630722 \\ \mathrm{H} & -1.470872 & -1.469679 & -1.370426 \\ \mathrm{C} & -5.129507 & -0.423383 & -2.418782 \\ \mathrm{H} & -6.082532 & -0.023342 & -2.053361 \\ \mathrm{H} & -4.510478 & 0.426164 & -2.729640 \\ \mathrm{H} & -5.330370 & -1.045424 & -3.298449 \\ \mathrm{C} & -5.343241 & -3.180946 & -0.253996 \\ \mathrm{H} & -4.877243 & -3.642620 & 0.623126 \\ \mathrm{H} & -6.353508 & -2.854185 & 0.016702 \\ \mathrm{H} & -5.426943 & -3.932130 & -1.047757\end{array}$

Frequencies (Top 10 out of 237)

1. 8.2044 cm-1 (Symmetry: A)

2. $\quad 11.4818 \mathrm{~cm}-1$ (Symmetry: A)

3. $\quad 16.5619 \mathrm{~cm}-1$ (Symmetry: A)

4. $22.5964 \mathrm{~cm}-1$ (Symmetry: A)

5. $\quad 28.2052 \mathrm{~cm}-1$ (Symmetry: A)

6. $\quad 31.6295 \mathrm{~cm}-1$ (Symmetry: A)

7. $\quad 41.1061 \mathrm{~cm}-1$ (Symmetry: A)

8. $\quad 45.9464 \mathrm{~cm}-1$ (Symmetry: A)

9. $\quad 53.0466 \mathrm{~cm}-1$ (Symmetry: A)

10. $59.2509 \mathrm{~cm}-1$ (Symmetry: A) 


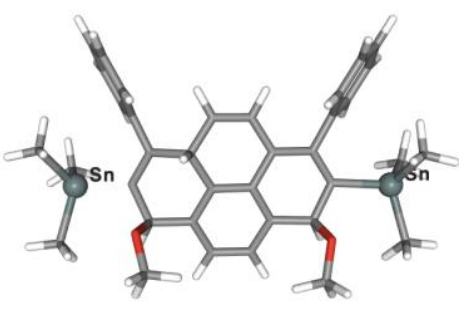

Charge

Multiplicity

Stoichiometry

Number of Basis Functions

0

Electronic Energy (Eh)

Number of Imaginary Frequencies

Mean of alpha and beta Electrons

2

$\mathrm{C} 36 \mathrm{H} 41 \mathrm{O} 2 \mathrm{Sn} 2$

440

$-1552.81884211$

0

140.5

Molecular Geometry in Cartesian Coordinates

$\begin{array}{lrrr}\mathrm{C} & -0.642519 & -2.965374 & 0.527654 \\ \mathrm{C} & 0.753429 & -2.981463 & 0.417225 \\ \mathrm{C} & -1.366322 & -1.813866 & 0.194589 \\ \mathrm{C} & 1.433680 & -1.849062 & -0.057269 \\ \mathrm{C} & 0.728043 & -0.677952 & -0.363005 \\ \mathrm{C} & -0.679559 & -0.637763 & -0.195583 \\ \mathrm{C} & -1.407856 & 0.617643 & -0.312491 \\ \mathrm{C} & -0.645021 & 1.817691 & -0.518477 \\ \mathrm{C} & 0.702499 & 1.806790 & -0.732599 \\ \mathrm{C} & 1.497374 & 0.531307 & -0.867009 \\ \mathrm{H} & -1.162512 & 2.770301 & -0.467898 \\ \mathrm{H} & 1.233842 & 2.745165 & -0.853694 \\ \mathrm{H} & 1.660175 & 0.362485 & -1.951335 \\ \mathrm{C} & 2.933982 & -1.870356 & -0.231319 \\ \mathrm{H} & 3.354783 & -2.671893 & 0.398542 \\ \mathrm{C} & 2.939649 & -3.493313 & -2.061652 \\ \mathrm{H} & 1.850631 & -3.596695 & -2.138848 \\ \mathrm{H} & 3.392854 & -3.632094 & -3.043844 \\ \mathrm{H} & 3.325157 & -4.254910 & -1.367058 \\ \mathrm{O} & 3.313197 & -2.164397 & -1.623234 \\ \mathrm{C} & 3.591513 & -0.532666 & 0.057290 \\ \mathrm{C} & 2.918908 & 0.593556 & -0.273156 \\ \mathrm{C} & 3.584518 & 1.926296 & -0.182318 \\ \mathrm{C} & 3.801120 & 2.698541 & -1.340975 \\ \mathrm{C} & 4.052590 & 2.407856 & 1.053794 \\ \mathrm{C} & 4.491602 & 3.914828 & -1.267966 \\ \mathrm{H} & 3.452408 & 2.328264 & -2.301833 \\ \mathrm{C} & 4.743544 & 3.627904 & 1.129686 \\ \mathrm{H} & 3.862967 & 1.827387 & 1.952643\end{array}$




\begin{tabular}{|c|c|c|c|}
\hline $\mathrm{C}$ & 4.968676 & 4.382626 & -0.031014 \\
\hline $\mathrm{H}$ & 4.665470 & 4.492357 & -2.170105 \\
\hline $\mathrm{H}$ & 5.099191 & 3.987143 & 2.090210 \\
\hline $\mathrm{H}$ & 5.504687 & 5.324339 & 0.025301 \\
\hline $\mathrm{Sn}$ & 5.681447 & -0.600933 & 0.505290 \\
\hline $\mathrm{C}$ & 6.101566 & -0.045946 & 2.524583 \\
\hline $\mathrm{H}$ & 5.263894 & -0.301811 & 3.183450 \\
\hline $\mathrm{H}$ & 6.995396 & -0.566769 & 2.887120 \\
\hline $\mathrm{H}$ & 6.279759 & 1.033295 & 2.592110 \\
\hline $\mathrm{C}$ & 6.203688 & -2.651738 & 0.236793 \\
\hline $\mathrm{H}$ & 5.840694 & -3.265742 & 1.069703 \\
\hline $\mathrm{H}$ & 5.758920 & -3.021293 & -0.694583 \\
\hline $\mathrm{H}$ & 7.291043 & -2.774123 & 0.174943 \\
\hline $\mathrm{C}$ & 6.754039 & 0.660208 & -0.828760 \\
\hline $\mathrm{H}$ & 7.826849 & 0.437130 & -0.802032 \\
\hline $\mathrm{H}$ & 6.392772 & 0.519552 & -1.853307 \\
\hline $\mathrm{H}$ & 6.608089 & 1.712224 & -0.554676 \\
\hline $\mathrm{H}$ & 1.316654 & -3.870029 & 0.691129 \\
\hline $\mathrm{H}$ & -1.171272 & -3.846427 & 0.882521 \\
\hline $\mathrm{C}$ & -2.876873 & -1.836840 & 0.223980 \\
\hline $\mathrm{H}$ & -3.209814 & -2.483486 & 1.055298 \\
\hline $\mathrm{C}$ & -3.207958 & -3.845812 & -1.180303 \\
\hline $\mathrm{H}$ & -2.160596 & -4.066304 & -1.417728 \\
\hline $\mathrm{H}$ & -3.499868 & -4.394240 & -0.271532 \\
\hline $\mathrm{H}$ & -3.842666 & -4.158251 & -2.011031 \\
\hline $\mathrm{C}$ & -3.547165 & -0.482804 & 0.276329 \\
\hline $\mathrm{C}$ & -2.828509 & 0.649800 & -0.043341 \\
\hline $\mathrm{C}$ & -3.553883 & 1.956150 & -0.150214 \\
\hline $\mathrm{C}$ & -3.662541 & 2.607501 & -1.394396 \\
\hline $\mathrm{C}$ & -4.183571 & 2.521960 & 0.972209 \\
\hline $\mathrm{C}$ & -4.399983 & 3.792499 & -1.516359 \\
\hline $\mathrm{H}$ & -3.183793 & 2.167211 & -2.264826 \\
\hline $\mathrm{C}$ & -4.924130 & 3.709327 & 0.852898 \\
\hline $\mathrm{H}$ & -4.085573 & 2.032433 & 1.937425 \\
\hline $\mathrm{C}$ & -5.037101 & 4.345731 & -0.392177 \\
\hline $\mathrm{H}$ & -4.485983 & 4.278121 & -2.483027 \\
\hline $\mathrm{H}$ & -5.405676 & 4.134621 & 1.727821 \\
\hline $\mathrm{H}$ & -5.611226 & 5.261690 & -0.486857 \\
\hline $\mathrm{O}$ & -3.426064 & -2.425362 & -1.017963 \\
\hline $\mathrm{Sn}$ & -5.681736 & -0.562615 & 0.428693 \\
\hline $\mathrm{C}$ & -6.234618 & -2.621026 & 0.392398 \\
\hline $\mathrm{H}$ & -5.607762 & -3.207445 & 1.076410 \\
\hline $\mathrm{H}$ & -7.280374 & -2.750034 & 0.695038 \\
\hline $\mathrm{H}$ & -6.099958 & -3.020205 & -0.617756 \\
\hline $\mathrm{C}$ & -6.571552 & 0.516829 & -1.172512 \\
\hline $\mathrm{H}$ & -6.487974 & 1.595247 & -0.990207 \\
\hline $\mathrm{H}$ & -6.060498 & 0.286647 & -2.113641 \\
\hline
\end{tabular}




$\begin{array}{lrrr}\mathrm{H} & -7.631837 & 0.259149 & -1.276165 \\ \mathrm{C} & -6.364443 & 0.235695 & 2.293400 \\ \mathrm{H} & -5.609991 & 0.105053 & 3.078029 \\ \mathrm{H} & -6.581647 & 1.305260 & 2.194496 \\ \mathrm{H} & -7.280697 & -0.276364 & 2.610622\end{array}$

Frequencies (Top 10 out of 237)

1. $\quad 13.5732 \mathrm{~cm}-1$ (Symmetry: A)

2. $\quad 17.8776 \mathrm{~cm}-1$ (Symmetry: A)

3. $\quad 33.1355 \mathrm{~cm}-1$ (Symmetry: A)

4. $\quad 37.0639 \mathrm{~cm}-1$ (Symmetry: A)

5. $\quad 42.1143 \mathrm{~cm}-1$ (Symmetry: A)

6. $\quad 45.3853 \mathrm{~cm}-1$ (Symmetry: A)

7. $\quad 55.0752 \mathrm{~cm}-1$ (Symmetry: A)

8. $\quad 57.1667 \mathrm{~cm}-1$ (Symmetry: A)

9. $\quad 58.0390 \mathrm{~cm}-1$ (Symmetry: A)

10. 64.1103 cm-1 (Symmetry: A)

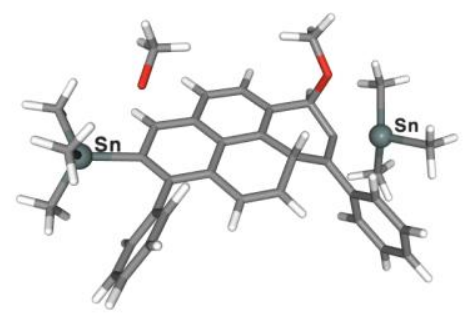

Charge

Multiplicity

Stoichiometry

Number of Basis Functions

0

Electronic Energy (Eh)

Number of Imaginary Frequencies

Mean of alpha and beta Electrons

2

$\mathrm{C} 36 \mathrm{H} 41 \mathrm{O} 2 \mathrm{Sn} 2$

440

$-1552.78698817$

1

140.5

Molecular Geometry in Cartesian Coordinates

$\begin{array}{lrrr}\mathrm{C} & 0.668224 & -2.931643 & -0.659133 \\ \mathrm{C} & -0.708294 & -2.958926 & -0.489161 \\ \mathrm{C} & 1.390613 & -1.738838 & -0.410393 \\ \mathrm{C} & -1.397683 & -1.812081 & -0.023334 \\ \mathrm{C} & -0.708057 & -0.630669 & 0.230975 \\ \mathrm{C} & 0.696934 & -0.567539 & 0.001605 \\ \mathrm{C} & 1.418873 & 0.673482 & 0.126388 \\ \mathrm{C} & 0.637647 & 1.884377 & 0.374886\end{array}$




\begin{tabular}{|c|c|c|c|}
\hline $\mathrm{C}$ & -0.691557 & 1.853865 & 0.617858 \\
\hline $\mathrm{C}$ & -1.470646 & 0.568701 & 0.764300 \\
\hline $\mathrm{H}$ & 1.150966 & 2.838640 & 0.318345 \\
\hline $\mathrm{H}$ & -1.232837 & 2.784272 & 0.758642 \\
\hline $\mathrm{H}$ & -1.595903 & 0.395214 & 1.851794 \\
\hline $\mathrm{C}$ & -2.889579 & -1.855049 & 0.206754 \\
\hline $\mathrm{H}$ & -3.321342 & -2.671036 & -0.396546 \\
\hline $\mathrm{C}$ & -2.790951 & -3.446421 & 2.062440 \\
\hline $\mathrm{H}$ & -1.697239 & -3.519798 & 2.104782 \\
\hline $\mathrm{H}$ & -3.206993 & -3.580718 & 3.061505 \\
\hline $\mathrm{H}$ & -3.178661 & -4.229755 & 1.393791 \\
\hline $\mathrm{O}$ & -3.214414 & -2.135310 & 1.615638 \\
\hline $\mathrm{C}$ & -3.577560 & -0.530170 & -0.074980 \\
\hline $\mathrm{C}$ & -2.911635 & 0.610272 & 0.218249 \\
\hline $\mathrm{C}$ & -3.596880 & 1.933022 & 0.133549 \\
\hline $\mathrm{C}$ & -3.786654 & 2.716762 & 1.289236 \\
\hline $\mathrm{C}$ & -4.110548 & 2.392993 & -1.092672 \\
\hline $\mathrm{C}$ & -4.494684 & 3.923316 & 1.223684 \\
\hline $\mathrm{H}$ & -3.404343 & 2.362192 & 2.243260 \\
\hline $\mathrm{C}$ & -4.819238 & 3.603248 & -1.161034 \\
\hline $\mathrm{H}$ & -3.943159 & 1.803308 & -1.989953 \\
\hline $\mathrm{C}$ & -5.016720 & 4.369629 & -0.002938 \\
\hline $\mathrm{H}$ & -4.647540 & 4.509596 & 2.123960 \\
\hline $\mathrm{H}$ & -5.210212 & 3.945884 & -2.113837 \\
\hline $\mathrm{H}$ & -5.566359 & 5.303761 & -0.053469 \\
\hline $\mathrm{Sn}$ & -5.680649 & -0.633886 & -0.450486 \\
\hline $\mathrm{C}$ & -6.175713 & -0.107641 & -2.460163 \\
\hline $\mathrm{H}$ & -5.359451 & -0.364133 & -3.145100 \\
\hline $\mathrm{H}$ & -7.077004 & -0.640225 & -2.785115 \\
\hline $\mathrm{H}$ & -6.365837 & 0.969147 & -2.533910 \\
\hline $\mathrm{C}$ & -6.162060 & -2.688134 & -0.138091 \\
\hline $\mathrm{H}$ & -5.826319 & -3.306612 & -0.979038 \\
\hline $\mathrm{H}$ & -5.672615 & -3.040616 & 0.777407 \\
\hline $\mathrm{H}$ & -7.243874 & -2.824753 & -0.028191 \\
\hline $\mathrm{C}$ & -6.721920 & 0.630112 & 0.905422 \\
\hline $\mathrm{H}$ & -7.790916 & 0.388741 & 0.924366 \\
\hline $\mathrm{H}$ & -6.318586 & 0.511637 & 1.917009 \\
\hline $\mathrm{H}$ & -6.605208 & 1.679993 & 0.609991 \\
\hline $\mathrm{H}$ & -1.271466 & -3.864104 & -0.703033 \\
\hline $\mathrm{H}$ & 1.204348 & -3.815269 & -0.994147 \\
\hline $\mathrm{C}$ & 2.825211 & -1.687518 & -0.543037 \\
\hline $\mathrm{H}$ & 3.314475 & -2.560179 & -0.974664 \\
\hline $\mathrm{C}$ & 2.900367 & -3.516727 & 1.637912 \\
\hline $\mathrm{H}$ & 1.811833 & -3.435151 & 1.761079 \\
\hline $\mathrm{H}$ & 3.123135 & -4.302429 & 0.899149 \\
\hline $\mathrm{H}$ & 3.335889 & -3.817753 & 2.601385 \\
\hline $\mathrm{C}$ & 3.526288 & -0.453973 & -0.472493 \\
\hline
\end{tabular}




$\begin{array}{lrrr}\mathrm{C} & 2.811332 & 0.712094 & -0.101745 \\ \mathrm{C} & 3.572723 & 1.981674 & 0.104548 \\ \mathrm{C} & 3.594921 & 2.598994 & 1.371224 \\ \mathrm{C} & 4.324185 & 2.548241 & -0.940355 \\ \mathrm{C} & 4.359435 & 3.752479 & 1.589619 \\ \mathrm{H} & 3.028605 & 2.154015 & 2.184770 \\ \mathrm{C} & 5.090681 & 3.704420 & -0.724752 \\ \mathrm{H} & 4.299060 & 2.086051 & -1.923549 \\ \mathrm{C} & 5.112845 & 4.308099 & 0.541465 \\ \mathrm{H} & 4.375791 & 4.210922 & 2.573095 \\ \mathrm{H} & 5.664314 & 4.130466 & -1.541881 \\ \mathrm{H} & 5.708056 & 5.199510 & 0.710747 \\ \mathrm{O} & 3.505980 & -2.257650 & 1.301686 \\ \mathrm{Sn} & 5.663450 & -0.634292 & -0.439298 \\ \mathrm{C} & 6.089256 & -2.702806 & -0.707927 \\ \mathrm{H} & 5.782389 & -3.046215 & -1.703463 \\ \mathrm{H} & 7.165251 & -2.887293 & -0.606501 \\ \mathrm{H} & 5.557713 & -3.282142 & 0.053585 \\ \mathrm{C} & 6.346744 & 0.136458 & 1.417027 \\ \mathrm{H} & 6.321716 & 1.232686 & 1.401623 \\ \mathrm{H} & 5.685606 & -0.219002 & 2.214438 \\ \mathrm{H} & 7.370419 & -0.189462 & 1.632668 \\ \mathrm{C} & 6.623585 & 0.385042 & -2.056844 \\ \mathrm{H} & 5.996710 & 0.382480 & -2.956517 \\ \mathrm{H} & 6.842511 & 1.423952 & -1.787227 \\ \mathrm{H} & 7.567593 & -0.118094 & -2.299178\end{array}$

Frequencies (Top 10 out of 237)

1. $\quad-516.2121 \mathrm{~cm}-1$ (Symmetry: A) *

2. $\quad 13.3052 \mathrm{~cm}-1$ (Symmetry: A)

3. $20.6049 \mathrm{~cm}-1$ (Symmetry: A)

4. $\quad 27.2241 \mathrm{~cm}-1$ (Symmetry: A)

5. $\quad 37.9144 \mathrm{~cm}-1$ (Symmetry: A)

6. $\quad 39.1208 \mathrm{~cm}-1$ (Symmetry: A)

7. $\quad 42.6092 \mathrm{~cm}-1$ (Symmetry: A)

8. $\quad 49.9913 \mathrm{~cm}-1$ (Symmetry: A)

9. $\quad 54.6814 \mathrm{~cm}-1$ (Symmetry: A)

10. $\quad 56.4669 \mathrm{~cm}-1$ (Symmetry: A) 


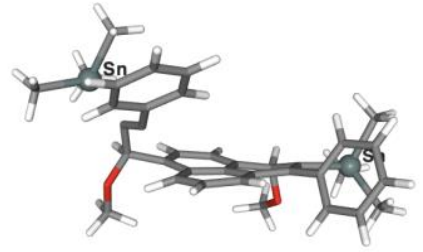

Charge

0

Multiplicity

2

Stoichiometry

Number of Basis Functions

$\mathrm{C} 36 \mathrm{H} 41 \mathrm{O} 2 \mathrm{Sn} 2$

440

Electronic Energy (Eh)

$-1552.76758591$

Number of Imaginary Frequencies

Mean of alpha and beta Electrons

1

140.5

Molecular Geometry in Cartesian Coordinates

$\begin{array}{lrrr}\mathrm{C} & 0.235112 & -2.925365 & -0.123963 \\ \mathrm{C} & -1.139792 & -2.896483 & 0.215572 \\ \mathrm{C} & 1.063190 & -1.852384 & 0.178276 \\ \mathrm{C} & -1.673827 & -1.811178 & 0.896372 \\ \mathrm{C} & -0.837754 & -0.710490 & 1.246075 \\ \mathrm{C} & 0.518901 & -0.698256 & 0.816433 \\ \mathrm{C} & 1.308349 & 0.499543 & 0.950012 \\ \mathrm{C} & 0.722774 & 1.625346 & 1.537590 \\ \mathrm{C} & -0.586377 & 1.587039 & 2.061255 \\ \mathrm{C} & -1.388816 & 0.444623 & 1.917040 \\ \mathrm{H} & 1.292755 & 2.545554 & 1.612918 \\ \mathrm{H} & -0.983082 & 2.456834 & 2.574434 \\ \mathrm{H} & -2.278078 & 0.320966 & 2.526459 \\ \mathrm{C} & -3.173817 & -1.687767 & 1.075611 \\ \mathrm{H} & -3.641669 & -2.603719 & 0.679615 \\ \mathrm{C} & -3.194499 & -2.653060 & 3.302536 \\ \mathrm{H} & -2.103774 & -2.696500 & 3.408899 \\ \mathrm{H} & -3.656361 & -2.486587 & 4.275955 \\ \mathrm{H} & -3.551651 & -3.603736 & 2.881331 \\ \mathrm{O} & -3.592265 & -1.538549 & 2.465421 \\ \mathrm{C} & -3.704151 & -0.456467 & 0.322653 \\ \mathrm{C} & -3.144678 & 0.733593 & 0.473195 \\ \mathrm{C} & -3.330749 & 2.122248 & 0.152840 \\ \mathrm{C} & -4.490974 & 2.809393 & 0.591909 \\ \mathrm{C} & -2.338190 & 2.835904 & -0.561332 \\ \mathrm{C} & -4.666213 & 4.164483 & 0.288516 \\ \mathrm{H} & -5.235305 & 2.269456 & 1.171478 \\ \mathrm{C} & -2.524417 & 4.188391 & -0.865320 \\ \mathrm{H} & -1.436580 & 2.315100 & -0.870570 \\ \mathrm{C} & -3.688305 & 4.857720 & -0.445874\end{array}$




\begin{tabular}{|c|c|c|c|}
\hline $\mathrm{H}$ & -5.560608 & 4.680521 & 0.622668 \\
\hline $\mathrm{H}$ & -1.763215 & 4.723995 & -1.423131 \\
\hline $\mathrm{H}$ & -3.826701 & 5.908021 & -0.680462 \\
\hline $\mathrm{Sn}$ & -5.394705 & -0.647994 & -0.989204 \\
\hline $\mathrm{C}$ & -5.123607 & 0.692982 & -2.614459 \\
\hline $\mathrm{H}$ & -4.156676 & 0.516744 & -3.098074 \\
\hline $\mathrm{H}$ & -5.914985 & 0.571897 & -3.362740 \\
\hline $\mathrm{H}$ & -5.139299 & 1.728635 & -2.256001 \\
\hline $\mathrm{C}$ & -5.417760 & -2.683267 & -1.629610 \\
\hline $\mathrm{H}$ & -4.424967 & -2.988601 & -1.980455 \\
\hline $\mathrm{H}$ & -5.716510 & -3.347042 & -0.810141 \\
\hline $\mathrm{H}$ & -6.127144 & -2.821343 & -2.453504 \\
\hline $\mathrm{C}$ & -7.178131 & -0.194624 & 0.085171 \\
\hline $\mathrm{H}$ & -8.053373 & -0.647782 & -0.393817 \\
\hline $\mathrm{H}$ & -7.106386 & -0.573083 & 1.110565 \\
\hline $\mathrm{H}$ & -7.331514 & 0.889604 & 0.126261 \\
\hline $\mathrm{H}$ & -1.786949 & -3.718535 & -0.080300 \\
\hline $\mathrm{H}$ & 0.640163 & -3.785337 & -0.651805 \\
\hline $\mathrm{C}$ & 2.540124 & -1.910666 & -0.139094 \\
\hline $\mathrm{H}$ & 2.684217 & -2.482896 & -1.072259 \\
\hline $\mathrm{C}$ & 3.035307 & -4.044323 & 1.000715 \\
\hline $\mathrm{H}$ & 2.037522 & -4.251829 & 1.404575 \\
\hline $\mathrm{H}$ & 3.135151 & -4.519073 & 0.012460 \\
\hline $\mathrm{H}$ & 3.791713 & -4.448185 & 1.675415 \\
\hline $\mathrm{C}$ & 3.242575 & -0.569688 & -0.197669 \\
\hline $\mathrm{C}$ & 2.673077 & 0.523375 & 0.384065 \\
\hline $\mathrm{C}$ & 3.459352 & 1.794437 & 0.480110 \\
\hline $\mathrm{C}$ & 3.894162 & 2.267971 & 1.733862 \\
\hline $\mathrm{C}$ & 3.827530 & 2.499637 & -0.679084 \\
\hline $\mathrm{C}$ & 4.692759 & 3.415436 & 1.823984 \\
\hline $\mathrm{H}$ & 3.621737 & 1.717825 & 2.630350 \\
\hline $\mathrm{C}$ & 4.629391 & 3.649636 & -0.591827 \\
\hline $\mathrm{H}$ & 3.482111 & 2.144550 & -1.646331 \\
\hline $\mathrm{C}$ & 5.065868 & 4.109092 & 0.659583 \\
\hline $\mathrm{H}$ & 5.031848 & 3.762281 & 2.794761 \\
\hline $\mathrm{H}$ & 4.908753 & 4.182078 & -1.495581 \\
\hline $\mathrm{H}$ & 5.687709 & 4.995715 & 0.729107 \\
\hline $\mathrm{O}$ & 3.288399 & -2.623008 & 0.917360 \\
\hline $\mathrm{Sn}$ & 5.281473 & -0.669375 & -0.840280 \\
\hline $\mathrm{C}$ & 5.729644 & -2.731210 & -1.146722 \\
\hline $\mathrm{H}$ & 4.985637 & -3.203158 & -1.801157 \\
\hline $\mathrm{H}$ & 6.714444 & -2.850436 & -1.613449 \\
\hline $\mathrm{H}$ & 5.722166 & -3.255389 & -0.185874 \\
\hline $\mathrm{C}$ & 6.564454 & 0.201818 & 0.614051 \\
\hline $\mathrm{H}$ & 6.499277 & 1.295676 & 0.563972 \\
\hline $\mathrm{H}$ & 6.266539 & -0.114185 & 1.619658 \\
\hline $\mathrm{H}$ & 7.605794 & -0.096123 & 0.446002 \\
\hline
\end{tabular}




$\begin{array}{lrrr}\mathrm{C} & 5.557582 & 0.323481 & -2.715927 \\ \mathrm{H} & 4.641899 & 0.289045 & -3.317770 \\ \mathrm{H} & 5.828566 & 1.373126 & -2.555074 \\ \mathrm{H} & 6.360827 & -0.158678 & -3.285717\end{array}$

Frequencies (Top 10 out of 237)

1. $-393.0286 \mathrm{~cm}-1$ (Symmetry: A) *

2. $\quad 11.3540 \mathrm{~cm}-1$ (Symmetry: A)

3. $\quad 15.6658 \mathrm{~cm}-1$ (Symmetry: A)

4. $\quad 18.9797 \mathrm{~cm}-1$ (Symmetry: A)

5. $\quad 20.2416 \mathrm{~cm}-1$ (Symmetry: A)

6. $\quad 30.1909 \mathrm{~cm}-1$ (Symmetry: A)

7. $\quad 35.5239 \mathrm{~cm}-1$ (Symmetry: A)

8. $\quad 40.8389 \mathrm{~cm}-1$ (Symmetry: A)

9. $\quad 47.8950 \mathrm{~cm}-1$ (Symmetry: A)

10. $\quad 53.0084 \mathrm{~cm}-1$ (Symmetry: A)

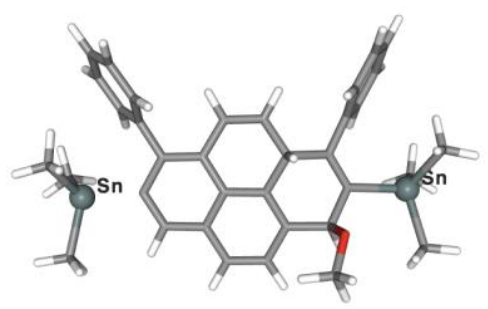

Charge

Multiplicity

Stoichiometry

Number of Basis Functions

Electronic Energy (Eh)

Number of Imaginary Frequencies

Mean of alpha and beta Electrons

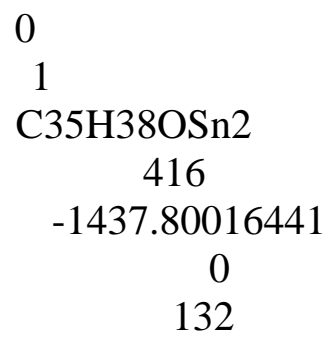

Molecular Geometry in Cartesian Coordinates

$\begin{array}{lrrr}\mathrm{C} & -0.640826 & -3.120290 & 0.150479 \\ \mathrm{C} & 0.731917 & -3.147960 & 0.297956 \\ \mathrm{C} & -1.307519 & -1.914258 & -0.203481 \\ \mathrm{C} & 1.491583 & -1.952284 & 0.141121 \\ \mathrm{C} & 0.823617 & -0.734374 & -0.177241 \\ \mathrm{C} & -0.590263 & -0.740354 & -0.382528 \\ \mathrm{C} & -1.325768 & 0.518563 & -0.812459 \\ \mathrm{C} & -0.503707 & 1.768007 & -0.588679\end{array}$




\begin{tabular}{|c|c|c|c|}
\hline $\mathrm{C}$ & 0.827417 & 1.742413 & -0.382003 \\
\hline $\mathrm{C}$ & 1.581362 & 0.486334 & -0.244424 \\
\hline $\mathrm{H}$ & -1.021147 & 2.720860 & -0.643136 \\
\hline $\mathrm{H}$ & 1.373274 & 2.673420 & -0.266107 \\
\hline $\mathrm{C}$ & 2.912054 & -1.941270 & 0.300625 \\
\hline $\mathrm{H}$ & 3.399576 & -2.894306 & 0.504967 \\
\hline $\mathrm{C}$ & 3.659185 & -0.777795 & 0.183946 \\
\hline $\mathrm{C}$ & 2.966260 & 0.459735 & -0.078185 \\
\hline $\mathrm{C}$ & 3.772778 & 1.714080 & -0.174100 \\
\hline $\mathrm{C}$ & 3.835590 & 2.442906 & -1.377425 \\
\hline $\mathrm{C}$ & 4.524167 & 2.160428 & 0.929523 \\
\hline $\mathrm{C}$ & 4.645226 & 3.582616 & -1.480840 \\
\hline $\mathrm{H}$ & 3.259931 & 2.099511 & -2.232527 \\
\hline $\mathrm{C}$ & 5.334726 & 3.301675 & 0.829869 \\
\hline $\mathrm{H}$ & 4.457957 & 1.619392 & 1.870160 \\
\hline $\mathrm{C}$ & 5.401802 & 4.013233 & -0.378138 \\
\hline $\mathrm{H}$ & 4.691767 & 4.128491 & -2.417607 \\
\hline $\mathrm{H}$ & 5.905126 & 3.635361 & 1.691029 \\
\hline $\mathrm{H}$ & 6.030851 & 4.893719 & -0.458714 \\
\hline $\mathrm{Sn}$ & 5.795750 & -0.929512 & 0.277902 \\
\hline $\mathrm{C}$ & 6.579965 & -0.312819 & 2.165460 \\
\hline $\mathrm{H}$ & 5.835360 & -0.441646 & 2.959258 \\
\hline $\mathrm{H}$ & 7.461287 & -0.911807 & 2.422427 \\
\hline $\mathrm{H}$ & 6.875970 & 0.741585 & 2.132008 \\
\hline $\mathrm{C}$ & 6.201553 & -3.011059 & 0.043498 \\
\hline $\mathrm{H}$ & 5.862542 & -3.580673 & 0.916838 \\
\hline $\mathrm{H}$ & 5.701545 & -3.411801 & -0.845591 \\
\hline $\mathrm{H}$ & 7.279439 & -3.176774 & -0.067840 \\
\hline $\mathrm{C}$ & 6.685595 & 0.194080 & -1.293083 \\
\hline $\mathrm{H}$ & 7.731676 & -0.099931 & -1.437619 \\
\hline $\mathrm{H}$ & 6.147609 & 0.036662 & -2.234495 \\
\hline $\mathrm{H}$ & 6.652198 & 1.264765 & -1.057243 \\
\hline $\mathrm{H}$ & 1.248069 & -4.070869 & 0.546684 \\
\hline $\mathrm{H}$ & -1.228777 & -4.023631 & 0.295025 \\
\hline $\mathrm{C}$ & -2.805443 & -1.897859 & -0.395103 \\
\hline $\mathrm{H}$ & -3.246226 & -2.741810 & 0.161667 \\
\hline $\mathrm{C}$ & -2.812531 & -3.356703 & -2.359282 \\
\hline $\mathrm{H}$ & -1.723634 & -3.462571 & -2.435014 \\
\hline $\mathrm{H}$ & -3.211396 & -4.172868 & -1.737971 \\
\hline $\mathrm{H}$ & -3.256742 & -3.405322 & -3.354166 \\
\hline $\mathrm{C}$ & -3.445230 & -0.577170 & -0.001929 \\
\hline $\mathrm{C}$ & -2.751883 & 0.561769 & -0.230778 \\
\hline $\mathrm{C}$ & -3.396407 & 1.893705 & -0.038562 \\
\hline $\mathrm{C}$ & -3.587921 & 2.760749 & -1.132889 \\
\hline $\mathrm{C}$ & -3.870255 & 2.281949 & 1.227796 \\
\hline $\mathrm{C}$ & -4.259380 & 3.978691 & -0.968593 \\
\hline $\mathrm{H}$ & -3.235452 & 2.462680 & -2.117196 \\
\hline
\end{tabular}




$\begin{array}{lrrr}\text { C } & -4.542038 & 3.503606 & 1.395353 \\ \text { H } & -3.700944 & 1.627245 & 2.078421 \\ \text { C } & -4.742231 & 4.353339 & 0.297454 \\ \text { H } & -4.414468 & 4.629660 & -1.822911 \\ \text { H } & -4.902528 & 3.790010 & 2.378305 \\ \text { H } & -5.263725 & 5.296329 & 0.424517 \\ \text { O } & -3.179802 & -2.067875 & -1.809807 \\ \text { Sn } & -5.540533 & -0.647557 & 0.416817 \\ \text { C } & -6.093608 & -2.661839 & -0.015686 \\ \text { H } & -5.756181 & -3.343674 & 0.774048 \\ \text { H } & -7.181589 & -2.759050 & -0.105052 \\ \text { H } & -5.638152 & -2.967645 & -0.964860 \\ \mathrm{C} & -6.576835 & 0.731546 & -0.826587 \\ \text { H } & -6.418968 & 1.756339 & -0.468771 \\ \text { H } & -6.203758 & 0.665897 & -1.854479 \\ \text { H } & -7.653119 & 0.524113 & -0.830646 \\ \mathrm{C} & -5.977260 & -0.241867 & 2.468042 \\ \text { H } & -5.155271 & -0.568339 & 3.115369 \\ \text { H } & -6.131368 & 0.832917 & 2.617115 \\ \text { H } & -6.888053 & -0.767567 & 2.777478 \\ \text { H } & -1.479274 & 0.428701 & -1.905652\end{array}$

Frequencies (Top 10 out of 222)

1. $\quad 13.1961 \mathrm{~cm}-1$ (Symmetry: A)

2. $\quad 20.0361 \mathrm{~cm}-1$ (Symmetry: A)

3. $\quad 35.3085 \mathrm{~cm}-1$ (Symmetry: A)

4. $\quad 40.6153 \mathrm{~cm}-1$ (Symmetry: A)

5. $\quad 45.2631 \mathrm{~cm}-1$ (Symmetry: A)

6. $\quad 52.3134 \mathrm{~cm}-1$ (Symmetry: A)

7. $\quad 54.7509 \mathrm{~cm}-1$ (Symmetry: A)

8. $\quad 60.0234 \mathrm{~cm}-1$ (Symmetry: A)

9. $\quad 61.1315 \mathrm{~cm}-1$ (Symmetry: A)

10. 63.1184 cm-1 (Symmetry: A)

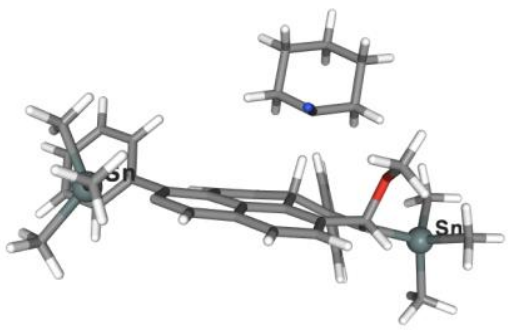

Charge 
Stoichiometry

Number of Basis Functions

Electronic Energy (Eh)

Number of Imaginary Frequencies

Mean of alpha and beta Electrons

\section{C42H48NOSn2}

508

$-1765.07336356$

1

161.5

Molecular Geometry in Cartesian Coordinates

$\begin{array}{lrrr}\mathrm{C} & 0.338692 & -3.296828 & -0.062375 \\ \mathrm{C} & -1.041479 & -3.325713 & -0.093933 \\ \mathrm{C} & 1.040581 & -2.062635 & -0.129994 \\ \mathrm{C} & -1.780134 & -2.115573 & -0.235504 \\ \mathrm{C} & -1.081900 & -0.881313 & -0.360194 \\ \mathrm{C} & 0.345425 & -0.869288 & -0.289606 \\ \mathrm{C} & 1.092940 & 0.431849 & -0.306676 \\ \mathrm{C} & 0.290080 & 1.576286 & -0.825074 \\ \mathrm{C} & -1.061984 & 1.541476 & -0.885927 \\ \mathrm{C} & -1.825005 & 0.329773 & -0.572761 \\ \mathrm{H} & 0.817064 & 2.476058 & -1.125050 \\ \mathrm{H} & -1.610411 & 2.413485 & -1.228233 \\ \mathrm{C} & -3.207881 & -2.106213 & -0.251634 \\ \mathrm{H} & -3.715230 & -3.060939 & -0.115687 \\ \mathrm{C} & -3.939636 & -0.934326 & -0.400673 \\ \mathrm{C} & -3.223879 & 0.301760 & -0.576619 \\ \mathrm{C} & -4.008914 & 1.561165 & -0.745955 \\ \mathrm{C} & -3.915932 & 2.599775 & 0.200913 \\ \mathrm{C} & -4.893201 & 1.710469 & -1.831103 \\ \mathrm{C} & -4.701131 & 3.754072 & 0.075914 \\ \mathrm{H} & -3.240589 & 2.484323 & 1.044510 \\ \mathrm{C} & -5.679727 & 2.865526 & -1.960368 \\ \mathrm{H} & -4.950405 & 0.925432 & -2.580951 \\ \mathrm{C} & -5.589672 & 3.888900 & -1.003907 \\ \mathrm{H} & -4.627656 & 4.540760 & 0.819867 \\ \mathrm{H} & -6.354365 & 2.967024 & -2.804660 \\ \mathrm{H} & -6.200497 & 4.780624 & -1.099609 \\ \mathrm{Sn} & -6.071712 & -1.040946 & -0.202863 \\ \mathrm{C} & -7.078182 & -0.940025 & -2.083441 \\ \mathrm{H} & -6.431544 & -1.297160 & -2.893084 \\ \mathrm{H} & -7.978532 & -1.564956 & -2.064098 \\ \mathrm{H} & -7.377489 & 0.090697 & -2.303996 \\ \mathrm{C} & -6.443776 & -2.962523 & 0.644221 \\ \mathrm{H} & -6.204790 & -3.761399 & -0.067679 \\ \mathrm{H} & -5.844581 & -3.115563 & 1.548884 \\ & -7.501489 & -3.059373 & 0.915341 \\ \mathrm{H} & -6.764054 & 0.502467 & 1.086158 \\ -7.784059 & 0.294965 & 1.429779\end{array}$




\begin{tabular}{|c|c|c|c|}
\hline $\mathrm{H}$ & -6.113805 & 0.591542 & 1.963488 \\
\hline $\mathrm{H}$ & -6.760758 & 1.464910 & 0.560056 \\
\hline $\mathrm{H}$ & -1.580753 & -4.264747 & -0.009522 \\
\hline $\mathrm{H}$ & 0.905220 & -4.219671 & 0.035077 \\
\hline $\mathrm{C}$ & 2.540245 & -2.036088 & 0.032270 \\
\hline $\mathrm{H}$ & 2.959242 & -2.990295 & -0.330445 \\
\hline $\mathrm{C}$ & 2.597826 & -3.087389 & 2.256520 \\
\hline $\mathrm{H}$ & 1.515233 & -3.202275 & 2.372901 \\
\hline $\mathrm{H}$ & 3.030492 & -3.992173 & 1.803546 \\
\hline $\mathrm{H}$ & 3.046653 & -2.917180 & 3.237017 \\
\hline $\mathrm{C}$ & 3.220330 & -0.853298 & -0.632347 \\
\hline $\mathrm{C}$ & 2.532708 & 0.309535 & -0.774363 \\
\hline $\mathrm{C}$ & 3.211323 & 1.523183 & -1.319779 \\
\hline $\mathrm{C}$ & 3.280224 & 2.724619 & -0.585849 \\
\hline $\mathrm{C}$ & 3.837837 & 1.467755 & -2.578933 \\
\hline $\mathrm{C}$ & 3.991456 & 3.825674 & -1.077885 \\
\hline $\mathrm{H}$ & 2.787449 & 2.790770 & 0.381134 \\
\hline $\mathrm{C}$ & 4.549637 & 2.570167 & -3.076834 \\
\hline $\mathrm{H}$ & 3.752870 & 0.560095 & -3.169706 \\
\hline $\mathrm{C}$ & 4.636666 & 3.750310 & -2.324107 \\
\hline $\mathrm{H}$ & 4.047626 & 4.737501 & -0.492061 \\
\hline $\mathrm{H}$ & 5.027892 & 2.507670 & -4.049214 \\
\hline $\mathrm{H}$ & 5.189537 & 4.602694 & -2.704803 \\
\hline $\mathrm{O}$ & 2.919813 & -1.919322 & 1.455143 \\
\hline $\mathrm{Sn}$ & 5.345652 & -1.034370 & -0.827509 \\
\hline $\mathrm{C}$ & 5.841290 & -2.867404 & 0.140594 \\
\hline $\mathrm{H}$ & 6.926556 & -2.962052 & 0.262188 \\
\hline $\mathrm{H}$ & 5.372218 & -2.897847 & 1.129837 \\
\hline $\mathrm{H}$ & 5.490680 & -3.728127 & -0.441822 \\
\hline $\mathrm{C}$ & 6.263214 & 0.634530 & 0.121699 \\
\hline $\mathrm{H}$ & 6.133515 & 1.535671 & -0.490220 \\
\hline $\mathrm{H}$ & 5.798832 & 0.811817 & 1.098655 \\
\hline $\mathrm{H}$ & 7.335544 & 0.464151 & 0.270933 \\
\hline $\mathrm{C}$ & 6.002816 & -1.172982 & -2.856112 \\
\hline $\mathrm{H}$ & 5.236637 & -1.637896 & -3.487395 \\
\hline $\mathrm{H}$ & 6.226169 & -0.177305 & -3.255633 \\
\hline $\mathrm{H}$ & 6.912042 & -1.782421 & -2.917615 \\
\hline $\mathrm{H}$ & 1.225881 & 0.654007 & 0.877098 \\
\hline $\mathrm{C}$ & 1.233951 & 0.844969 & 2.483056 \\
\hline $\mathrm{C}$ & 2.709492 & 0.946072 & 2.828225 \\
\hline $\mathrm{C}$ & 0.453632 & 2.147397 & 2.555254 \\
\hline $\mathrm{C}$ & 0.550258 & -0.344491 & 2.867442 \\
\hline $\mathrm{C}$ & 2.860141 & 1.585253 & 4.232523 \\
\hline $\mathrm{H}$ & 3.210533 & 1.592320 & 2.092566 \\
\hline $\mathrm{H}$ & 3.181848 & -0.037304 & 2.767575 \\
\hline $\mathrm{C}$ & 0.629357 & 2.779461 & 3.958820 \\
\hline $\mathrm{H}$ & 0.847645 & 2.842939 & 1.797947 \\
\hline
\end{tabular}




$\begin{array}{lccc}\mathrm{H} & -0.604487 & 1.983454 & 2.324958 \\ \mathrm{~N} & -0.032001 & -1.335002 & 3.152458 \\ \mathrm{C} & 2.122542 & 2.935604 & 4.303572 \\ \mathrm{H} & 3.924000 & 1.715699 & 4.461788 \\ \mathrm{H} & 2.444119 & 0.900767 & 4.984395 \\ \mathrm{H} & 0.122552 & 3.750917 & 3.990225 \\ \mathrm{H} & 0.147237 & 2.132840 & 4.704259 \\ \mathrm{H} & 2.231588 & 3.372298 & 5.302932 \\ \mathrm{H} & 2.585988 & 3.636879 & 3.593413\end{array}$

Frequencies (Top 10 out of 276)

1. $-856.1431 \mathrm{~cm}-1$ (Symmetry: A) *

2. $\quad 14.9859 \mathrm{~cm}-1$ (Symmetry: A)

3. $\quad 18.8590 \mathrm{~cm}-1$ (Symmetry: A)

4. $22.1498 \mathrm{~cm}-1$ (Symmetry: A)

5. $\quad 32.3762 \mathrm{~cm}-1$ (Symmetry: A)

6. $\quad 37.4431 \mathrm{~cm}-1$ (Symmetry: A)

7. $\quad 44.2869 \mathrm{~cm}-1$ (Symmetry: A)

8. $\quad 48.0758 \mathrm{~cm}-1$ (Symmetry: A)

9. $50.0848 \mathrm{~cm}-1$ (Symmetry: A)

10. $55.4650 \mathrm{~cm}-1$ (Symmetry: A)

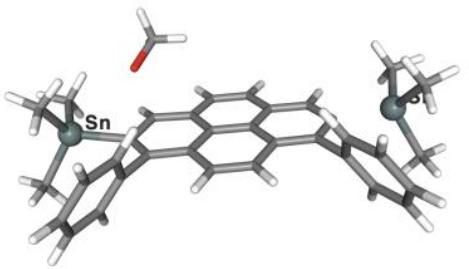

Charge

Multiplicity

Stoichiometry

Number of Basis Functions

0

Electronic Energy (Eh)

Number of Imaginary Frequencies

Mean of alpha and beta Electrons

2

C35H37OSn2

414

$-1437.17731039$

1

131.5

Molecular Geometry in Cartesian Coordinates

$\begin{array}{lrrr}\text { C } & 0.616761 & -3.096826 & -0.492940 \\ \text { C } & -0.755066 & -3.131275 & -0.473846 \\ \text { C } & 1.324415 & -1.852714 & -0.394246 \\ \text { C } & -1.522099 & -1.916974 & -0.356940\end{array}$




\begin{tabular}{|c|c|c|c|}
\hline $\mathrm{C}$ & -0.839366 & -0.667458 & -0.271546 \\
\hline $\mathrm{C}$ & 0.593265 & -0.634797 & -0.296094 \\
\hline $\mathrm{C}$ & 1.291696 & 0.610324 & -0.245294 \\
\hline $\mathrm{C}$ & 0.510812 & 1.817221 & -0.192112 \\
\hline $\mathrm{C}$ & -0.860990 & 1.788073 & -0.152440 \\
\hline $\mathrm{C}$ & -1.591489 & 0.542677 & -0.169900 \\
\hline $\mathrm{H}$ & 1.026616 & 2.771079 & -0.204676 \\
\hline $\mathrm{H}$ & -1.419065 & 2.718073 & -0.132946 \\
\hline $\mathrm{C}$ & -2.932441 & -1.931136 & -0.328719 \\
\hline $\mathrm{H}$ & -3.430834 & -2.898629 & -0.382669 \\
\hline $\mathrm{C}$ & -3.693541 & -0.753153 & -0.214263 \\
\hline $\mathrm{C}$ & -3.011583 & 0.487759 & -0.132482 \\
\hline $\mathrm{C}$ & -3.810063 & 1.741954 & 0.015171 \\
\hline $\mathrm{C}$ & -4.698435 & 2.141932 & -1.000597 \\
\hline $\mathrm{C}$ & -3.727307 & 2.514454 & 1.190032 \\
\hline $\mathrm{C}$ & -5.501100 & 3.282233 & -0.840627 \\
\hline $\mathrm{H}$ & -4.746555 & 1.565625 & -1.921045 \\
\hline $\mathrm{C}$ & -4.529083 & 3.652382 & 1.353268 \\
\hline $\mathrm{H}$ & -3.047322 & 2.205778 & 1.979291 \\
\hline $\mathrm{C}$ & -5.422112 & 4.037854 & 0.339290 \\
\hline $\mathrm{H}$ & -6.178960 & 3.580344 & -1.634178 \\
\hline $\mathrm{H}$ & -4.463490 & 4.232039 & 2.268248 \\
\hline $\mathrm{H}$ & -6.044275 & 4.917669 & 0.466401 \\
\hline $\mathrm{Sn}$ & -5.823395 & -0.938008 & -0.027376 \\
\hline $\mathrm{C}$ & -6.837076 & -0.449665 & -1.840656 \\
\hline $\mathrm{H}$ & -6.188677 & -0.616879 & -2.708192 \\
\hline $\mathrm{H}$ & -7.730434 & -1.074194 & -1.955718 \\
\hline $\mathrm{H}$ & -7.148198 & 0.600892 & -1.834859 \\
\hline $\mathrm{C}$ & -6.149440 & -3.005598 & 0.384994 \\
\hline $\mathrm{H}$ & -5.900419 & -3.627024 & -0.483299 \\
\hline $\mathrm{H}$ & -5.542356 & -3.339211 & 1.234355 \\
\hline $\mathrm{H}$ & -7.203131 & -3.180679 & 0.631272 \\
\hline $\mathrm{C}$ & -6.534676 & 0.269848 & 1.570937 \\
\hline $\mathrm{H}$ & -7.544638 & -0.035083 & 1.868458 \\
\hline $\mathrm{H}$ & -5.876130 & 0.185625 & 2.442488 \\
\hline $\mathrm{H}$ & -6.562992 & 1.323069 & 1.266377 \\
\hline $\mathrm{H}$ & -1.288392 & -4.074727 & -0.549850 \\
\hline $\mathrm{H}$ & 1.191915 & -4.014092 & -0.586103 \\
\hline $\mathrm{C}$ & 2.750211 & -1.809283 & -0.358658 \\
\hline $\mathrm{H}$ & 3.283526 & -2.730679 & -0.591004 \\
\hline $\mathrm{C}$ & 2.556536 & -3.270807 & 2.085164 \\
\hline $\mathrm{H}$ & 1.461589 & -3.191749 & 2.079968 \\
\hline $\mathrm{H}$ & 2.859961 & -4.166085 & 1.520785 \\
\hline $\mathrm{H}$ & 2.888951 & -3.391089 & 3.125966 \\
\hline $\mathrm{C}$ & 3.457097 & -0.558320 & -0.406099 \\
\hline $\mathrm{C}$ & 2.728279 & 0.626330 & -0.302062 \\
\hline $\mathrm{C}$ & 3.461193 & 1.923679 & -0.179728 \\
\hline
\end{tabular}




$\begin{array}{lrrr}\mathrm{C} & 4.303140 & 2.375064 & -1.211390 \\ \mathrm{C} & 3.365601 & 2.683916 & 1.003280 \\ \mathrm{C} & 5.043947 & 3.558857 & -1.063480 \\ \mathrm{H} & 4.370193 & 1.799868 & -2.130881 \\ \mathrm{C} & 4.103476 & 3.865155 & 1.153562 \\ \mathrm{H} & 2.730811 & 2.327563 & 1.809945 \\ \mathrm{C} & 4.947743 & 4.305877 & 0.119861 \\ \mathrm{H} & 5.689367 & 3.894518 & -1.869074 \\ \mathrm{H} & 4.029383 & 4.434349 & 2.074545 \\ \mathrm{H} & 5.521819 & 5.219287 & 0.236824 \\ \mathrm{O} & 3.191164 & -2.075119 & 1.601352 \\ \mathrm{Sn} & 5.576207 & -0.705878 & -0.122180 \\ \mathrm{C} & 6.013940 & -2.786434 & -0.002091 \\ \mathrm{H} & 5.818010 & -3.290616 & -0.956315 \\ \mathrm{H} & 7.069983 & -2.941154 & 0.248591 \\ \mathrm{H} & 5.395553 & -3.240667 & 0.778833 \\ \mathrm{C} & 6.088687 & 0.354344 & 1.644845 \\ \mathrm{H} & 6.083372 & 1.433819 & 1.451643 \\ \mathrm{H} & 5.349406 & 0.134178 & 2.422351 \\ \mathrm{H} & 7.081742 & 0.066682 & 2.008012 \\ \mathrm{C} & 6.692303 & 0.056179 & -1.780204 \\ \mathrm{H} & 6.154019 & -0.095119 & -2.723371 \\ \mathrm{H} & 6.885541 & 1.127487 & -1.657140 \\ \mathrm{H} & 7.654762 & -0.465023 & -1.849970\end{array}$

Frequencies (Top 10 out of 219)

1. $-534.7498 \mathrm{~cm}-1$ (Symmetry: A) *

2. $\quad 18.7689 \mathrm{~cm}-1$ (Symmetry: A)

3. $\quad 21.7656 \mathrm{~cm}-1$ (Symmetry: A)

4. $\quad 28.2702 \mathrm{~cm}-1$ (Symmetry: A)

5. $42.2864 \mathrm{~cm}-1$ (Symmetry: A)

6. $\quad 44.4399 \mathrm{~cm}-1$ (Symmetry: A)

7. $\quad 48.4282 \mathrm{~cm}-1$ (Symmetry: A)

8. $\quad 49.8908 \mathrm{~cm}-1$ (Symmetry: A)

9. $\quad 56.2007 \mathrm{~cm}-1$ (Symmetry: A)

10. $60.7916 \mathrm{~cm}-1$ (Symmetry: A) 


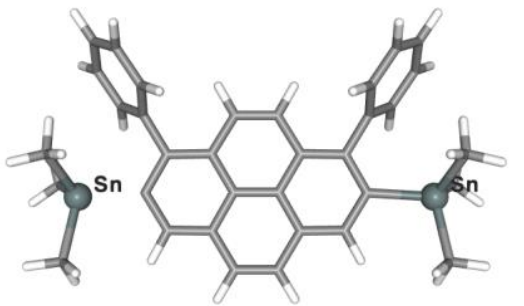

Charge

Multiplicity

Stoichiometry

Number of Basis Functions

Electronic Energy (Eh)

Number of Imaginary Frequencies

Mean of alpha and beta Electrons
0

1

C34H34Sn2
390
-1322.19083237
0
123

Molecular Geometry in Cartesian Coordinates

$\begin{array}{lrrr}\mathrm{C} & -0.682265 & -3.282671 & 0.018746 \\ \mathrm{C} & 0.682266 & -3.282671 & 0.018746 \\ \mathrm{C} & -1.426323 & -2.041794 & 0.044608 \\ \mathrm{C} & 1.426323 & -2.041794 & 0.044609 \\ \mathrm{C} & 0.717891 & -0.804583 & 0.062854 \\ \mathrm{C} & -0.717890 & -0.804583 & 0.062854 \\ \mathrm{C} & -1.443401 & 0.425644 & 0.088032 \\ \mathrm{C} & -0.682789 & 1.658112 & 0.157802 \\ \mathrm{C} & 0.682789 & 1.658112 & 0.157802 \\ \mathrm{C} & 1.443401 & 0.425644 & 0.088032 \\ \mathrm{H} & -1.223307 & 2.595630 & 0.233579 \\ \mathrm{H} & 1.223307 & 2.595630 & 0.233579 \\ \mathrm{C} & 2.833900 & -2.024351 & 0.048667 \\ \mathrm{H} & 3.353678 & -2.981621 & 0.021605 \\ \mathrm{C} & 3.569197 & -0.824386 & 0.068320 \\ \mathrm{C} & 2.861554 & 0.404537 & 0.084266 \\ \mathrm{C} & 3.637468 & 1.682292 & 0.081571 \\ \mathrm{C} & 4.475941 & 2.003088 & 1.165572 \\ \mathrm{C} & 3.587319 & 2.557425 & -1.020938 \\ \mathrm{C} & 5.262867 & 3.165145 & 1.142935 \\ \mathrm{H} & 4.498675 & 1.345407 & 2.030779 \\ \mathrm{C} & 4.372522 & 3.718252 & -1.046972 \\ \mathrm{H} & 2.945939 & 2.310779 & -1.862629 \\ \mathrm{C} & 5.216952 & 4.023715 & 0.033792 \\ \mathrm{H} & 5.902996 & 3.399847 & 1.987534 \\ \mathrm{H} & 4.332757 & 4.377486 & -1.908065 \\ \mathrm{H} & 5.827209 & 4.920678 & 0.012134 \\ \mathrm{Sn} & 5.706689 & -0.945622 & -0.059956 \\ \mathrm{C} & 6.648478 & -0.604210 & 1.825117\end{array}$




\begin{tabular}{|c|c|c|c|}
\hline $\mathrm{H}$ & 5.975850 & -0.863019 & 2.650725 \\
\hline $\mathrm{H}$ & 7.552320 & -1.217865 & 1.914451 \\
\hline $\mathrm{H}$ & 6.934067 & 0.448957 & 1.924632 \\
\hline $\mathrm{C}$ & 6.103465 & -2.957820 & -0.647896 \\
\hline $\mathrm{H}$ & 5.839104 & -3.662457 & 0.149410 \\
\hline $\mathrm{H}$ & 5.536774 & -3.225292 & -1.547083 \\
\hline $\mathrm{H}$ & 7.169880 & -3.084424 & -0.867860 \\
\hline $\mathrm{C}$ & 6.446833 & 0.421267 & -1.510902 \\
\hline $\mathrm{H}$ & 7.474825 & 0.167638 & -1.794707 \\
\hline $\mathrm{H}$ & 5.823834 & 0.404938 & -2.412004 \\
\hline $\mathrm{H}$ & 6.436487 & 1.441597 & -1.108572 \\
\hline $\mathrm{H}$ & 1.239519 & -4.215152 & 0.000383 \\
\hline $\mathrm{H}$ & -1.239519 & -4.215152 & 0.000383 \\
\hline $\mathrm{C}$ & -2.833899 & -2.024351 & 0.048666 \\
\hline $\mathrm{H}$ & -3.353678 & -2.981622 & 0.021604 \\
\hline $\mathrm{C}$ & -3.569197 & -0.824386 & 0.068319 \\
\hline $\mathrm{C}$ & -2.861554 & 0.404537 & 0.084266 \\
\hline $\mathrm{C}$ & -3.637468 & 1.682292 & 0.081573 \\
\hline $\mathrm{C}$ & -4.475941 & 2.003086 & 1.165574 \\
\hline $\mathrm{C}$ & -3.587319 & 2.557427 & -1.020936 \\
\hline $\mathrm{C}$ & -5.262866 & 3.165144 & 1.142939 \\
\hline $\mathrm{H}$ & -4.498675 & 1.345404 & 2.030780 \\
\hline $\mathrm{C}$ & -4.372523 & 3.718253 & -1.046967 \\
\hline $\mathrm{H}$ & -2.945940 & 2.310782 & -1.862627 \\
\hline $\mathrm{C}$ & -5.216952 & 4.023715 & 0.033798 \\
\hline $\mathrm{H}$ & -5.902995 & 3.399844 & 1.987539 \\
\hline $\mathrm{H}$ & -4.332758 & 4.377489 & -1.908059 \\
\hline $\mathrm{H}$ & -5.827209 & 4.920678 & 0.012141 \\
\hline $\mathrm{Sn}$ & -5.706689 & -0.945622 & -0.059957 \\
\hline $\mathrm{C}$ & -6.103465 & -2.957819 & -0.647898 \\
\hline $\mathrm{H}$ & -5.839103 & -3.662457 & 0.149406 \\
\hline $\mathrm{H}$ & -7.169880 & -3.084424 & -0.867862 \\
\hline $\mathrm{H}$ & -5.536774 & -3.225291 & -1.547086 \\
\hline $\mathrm{C}$ & -6.446833 & 0.421268 & -1.510902 \\
\hline $\mathrm{H}$ & -6.436484 & 1.441598 & -1.108572 \\
\hline $\mathrm{H}$ & -5.823836 & 0.404938 & -2.412005 \\
\hline $\mathrm{H}$ & -7.474826 & 0.167641 & -1.794705 \\
\hline $\mathrm{C}$ & -6.648479 & -0.604212 & 1.825116 \\
\hline $\mathrm{H}$ & -5.975852 & -0.863024 & 2.650723 \\
\hline $\mathrm{H}$ & -6.934065 & 0.448956 & 1.924633 \\
\hline $\mathrm{H}$ & -7.552322 & -1.217864 & 1.914448 \\
\hline
\end{tabular}

Frequencies (Top 10 out of 204)

1. $\quad 19.7446 \mathrm{~cm}-1$ (Symmetry: A) 
2. $\quad 20.2178 \mathrm{~cm}-1$ (Symmetry: A)

3. $40.7188 \mathrm{~cm}-1$ (Symmetry: A)

4. $\quad 44.9887 \mathrm{~cm}-1$ (Symmetry: A)

5. $\quad 49.0905 \mathrm{~cm}-1$ (Symmetry: A)

6. $\quad 54.9360 \mathrm{~cm}-1$ (Symmetry: A)

7. $56.4240 \mathrm{~cm}-1$ (Symmetry: A)

8. $\quad 61.0533 \mathrm{~cm}-1$ (Symmetry: A)

9. $\quad 62.0260 \mathrm{~cm}-1$ (Symmetry: A)

10. $76.1827 \mathrm{~cm}-1$ (Symmetry: A)

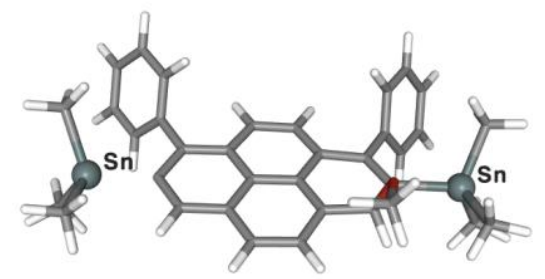

Charge

Multiplicity

Stoichiometry

Number of Basis Functions

0

2

Electronic Energy (Eh)

C35H37OSn2

414

Number of Imaginary Frequencies

Mean of alpha and beta Electrons

$-1437.2067472699998$

0

131.5

Molecular Geometry in Cartesian Coordinates

$\begin{array}{lrrr}\mathrm{C} & -0.595031 & -3.099673 & 0.388768 \\ \mathrm{C} & 0.795352 & -3.131543 & 0.440169 \\ \mathrm{C} & -1.290131 & -1.890588 & 0.189336 \\ \mathrm{C} & 1.548785 & -1.931636 & 0.300983 \\ \mathrm{C} & 0.859257 & -0.690309 & 0.149080 \\ \mathrm{C} & -0.571398 & -0.669989 & 0.113897 \\ \mathrm{C} & -1.270339 & 0.583147 & 0.058685 \\ \mathrm{C} & -0.511930 & 1.773984 & 0.013376 \\ \mathrm{C} & 0.878547 & 1.757670 & -0.004722 \\ \mathrm{C} & 1.608762 & 0.527928 & 0.053870 \\ \mathrm{H} & -1.027821 & 2.728058 & 0.013122 \\ \mathrm{H} & 1.424891 & 2.694217 & -0.027292 \\ \mathrm{C} & 2.972056 & -1.932475 & 0.326759 \\ \mathrm{H} & 3.472371 & -2.895103 & 0.427672 \\ \mathrm{C} & 3.724104 & -0.755173 & 0.205785 \\ \mathrm{C} & 3.036113 & 0.480853 & 0.067131 \\ \mathrm{C} & 3.828882 & 1.736274 & -0.086190 \\ \mathrm{C} & 3.767245 & 2.487289 & -1.276752 \\ \mathrm{C} & 4.693171 & 2.161331 & 0.940580\end{array}$




\begin{tabular}{|c|c|c|c|}
\hline $\mathrm{C}$ & 4.566853 & 3.625903 & -1.444434 \\
\hline $\mathrm{H}$ & 3.105982 & 2.159995 & -2.074356 \\
\hline $\mathrm{C}$ & 5.493299 & 3.302782 & 0.776284 \\
\hline $\mathrm{H}$ & 4.723696 & 1.603614 & 1.873166 \\
\hline $\mathrm{C}$ & 5.436234 & 4.035422 & -0.419213 \\
\hline $\mathrm{H}$ & 4.518197 & 4.187588 & -2.371645 \\
\hline $\mathrm{H}$ & 6.152056 & 3.619711 & 1.578636 \\
\hline $\mathrm{H}$ & 6.056745 & 4.915910 & -0.549859 \\
\hline $\mathrm{Sn}$ & 5.859199 & -0.929568 & 0.086368 \\
\hline $\mathrm{C}$ & 6.822892 & -0.382402 & 1.910402 \\
\hline $\mathrm{H}$ & 6.149019 & -0.516504 & 2.764193 \\
\hline $\mathrm{H}$ & 7.709422 & -1.006126 & 2.072917 \\
\hline $\mathrm{H}$ & 7.139233 & 0.666070 & 1.876613 \\
\hline $\mathrm{C}$ & 6.212695 & -3.005493 & -0.256226 \\
\hline $\mathrm{H}$ & 5.941904 & -3.603275 & 0.622116 \\
\hline $\mathrm{H}$ & 5.633462 & -3.368236 & -1.113015 \\
\hline $\mathrm{H}$ & 7.274576 & -3.179057 & -0.465766 \\
\hline $\mathrm{C}$ & 6.604337 & 0.237166 & -1.527325 \\
\hline $\mathrm{H}$ & 7.625265 & -0.065467 & -1.787440 \\
\hline $\mathrm{H}$ & 5.971045 & 0.119444 & -2.413609 \\
\hline $\mathrm{H}$ & 6.613253 & 1.299334 & -1.254141 \\
\hline $\mathrm{H}$ & 1.321675 & -4.070101 & 0.588072 \\
\hline $\mathrm{H}$ & -1.160464 & -4.021101 & 0.503964 \\
\hline $\mathrm{C}$ & -2.789165 & -1.898435 & 0.018382 \\
\hline $\mathrm{H}$ & -3.222081 & -2.668432 & 0.681536 \\
\hline $\mathrm{C}$ & -2.920576 & -3.635183 & -1.740614 \\
\hline $\mathrm{H}$ & -1.849784 & -3.816518 & -1.888462 \\
\hline $\mathrm{H}$ & -3.308845 & -4.330985 & -0.981078 \\
\hline $\mathrm{H}$ & -3.449050 & -3.794999 & -2.681762 \\
\hline $\mathrm{C}$ & -3.470092 & -0.559107 & 0.205390 \\
\hline $\mathrm{C}$ & -2.738023 & 0.592469 & 0.147081 \\
\hline $\mathrm{C}$ & -3.446775 & 1.911755 & 0.148229 \\
\hline $\mathrm{C}$ & -3.448215 & 2.719234 & -1.006181 \\
\hline $\mathrm{C}$ & -4.168996 & 2.336022 & 1.277224 \\
\hline $\mathrm{C}$ & -4.169632 & 3.919819 & -1.034880 \\
\hline $\mathrm{H}$ & -2.901429 & 2.387332 & -1.884614 \\
\hline $\mathrm{C}$ & -4.894535 & 3.538366 & 1.250738 \\
\hline $\mathrm{H}$ & -4.157070 & 1.723095 & 2.174447 \\
\hline $\mathrm{C}$ & -4.898663 & 4.331967 & 0.094081 \\
\hline $\mathrm{H}$ & -4.172800 & 4.526515 & -1.934634 \\
\hline $\mathrm{H}$ & -5.449663 & 3.852423 & 2.129018 \\
\hline $\mathrm{H}$ & -5.460645 & 5.259977 & 0.070836 \\
\hline $\mathrm{O}$ & -3.178573 & -2.262656 & -1.363119 \\
\hline $\mathrm{Sn}$ & -5.609217 & -0.635350 & 0.119050 \\
\hline $\mathrm{C}$ & -6.141941 & -2.657593 & -0.292615 \\
\hline $\mathrm{H}$ & -5.619308 & -3.347468 & 0.382255 \\
\hline $\mathrm{H}$ & -7.219976 & -2.810569 & -0.165076 \\
\hline
\end{tabular}




$\begin{array}{lrrr}\mathrm{H} & -5.862389 & -2.909000 & -1.320492 \\ \mathrm{C} & -6.337547 & 0.686803 & -1.377862 \\ \mathrm{H} & -6.283471 & 1.722693 & -1.021319 \\ \mathrm{H} & -5.730206 & 0.603831 & -2.285660 \\ \mathrm{H} & -7.378989 & 0.455756 & -1.629572 \\ \mathrm{C} & -6.476163 & -0.121215 & 2.006240 \\ \mathrm{H} & -5.808914 & -0.391484 & 2.832969 \\ \mathrm{H} & -6.671468 & 0.955992 & 2.055305 \\ \mathrm{H} & -7.425494 & -0.652240 & 2.144274\end{array}$

Frequencies (Top 10 out of 219)

1. $\quad 17.3564 \mathrm{~cm}-1$ (Symmetry: A)

2. $\quad 21.0099 \mathrm{~cm}-1$ (Symmetry: A)

3. $\quad 34.6526 \mathrm{~cm}-1$ (Symmetry: A)

4. $\quad 36.6882 \mathrm{~cm}-1$ (Symmetry: A)

5. $\quad 44.3635 \mathrm{~cm}-1$ (Symmetry: A)

6. $\quad 50.2690 \mathrm{~cm}-1$ (Symmetry: A)

7. $\quad 50.6357 \mathrm{~cm}-1$ (Symmetry: A)

8. $\quad 59.1047 \mathrm{~cm}-1$ (Symmetry: A)

9. $\quad 60.8942 \mathrm{~cm}-1$ (Symmetry: A)

10. $\quad 65.2476 \mathrm{~cm}-1$ (Symmetry: A) 


\section{SPECTROSCOPIC DATA}

\section{${ }^{1} \mathrm{H}$ NMR of precursor $1 \mathrm{a}$}

$\mathrm{CDCl}_{3}, 25^{\circ} \mathrm{C}$
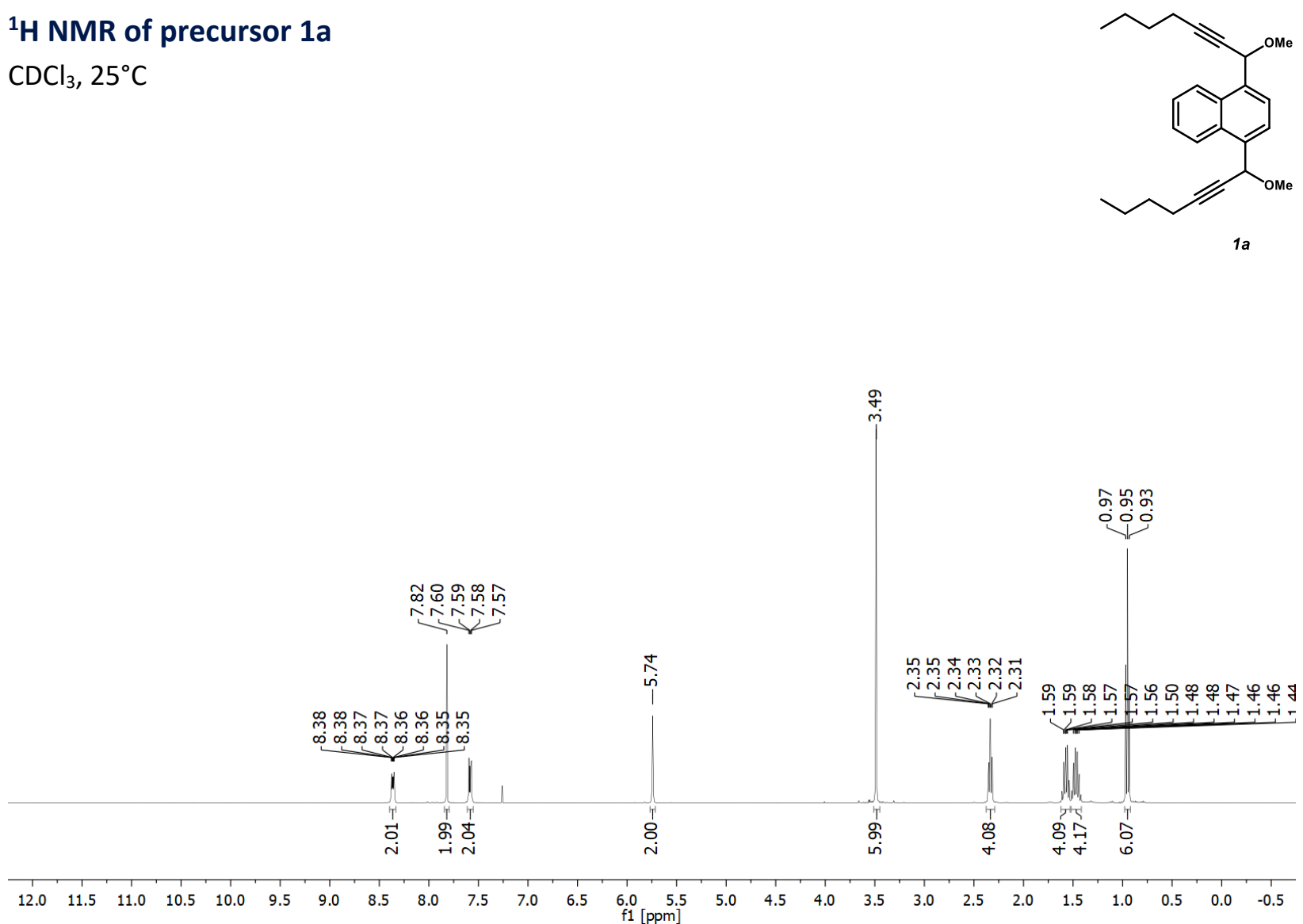

\section{${ }^{13} \mathrm{C}$ NMR of precursor $1 \mathrm{a}$}

$\mathrm{CDCl}_{3}, 25^{\circ} \mathrm{C}$

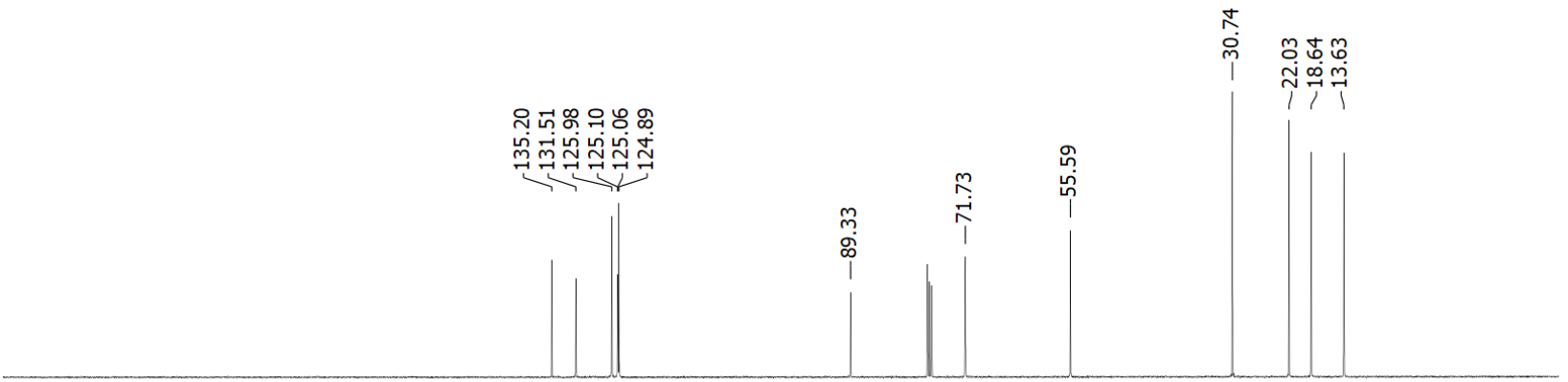

$\begin{array}{llllllllllllllllllllllllll}210 & 200 & 190 & 180 & 170 & 160 & 150 & 140 & 130 & 120 & 110 & \begin{array}{c}100 \\ \mathrm{f} 1[\mathrm{ppm}]\end{array} & 90 & 80 & 70 & 60 & 50 & 40 & 30 & 20 & 10 & 0 & -10 & \end{array}$




\section{${ }^{1} \mathrm{H}$ NMR of precursor $1 \mathrm{~b}$}

$\mathrm{CDCl}_{3}, 25^{\circ} \mathrm{C}$
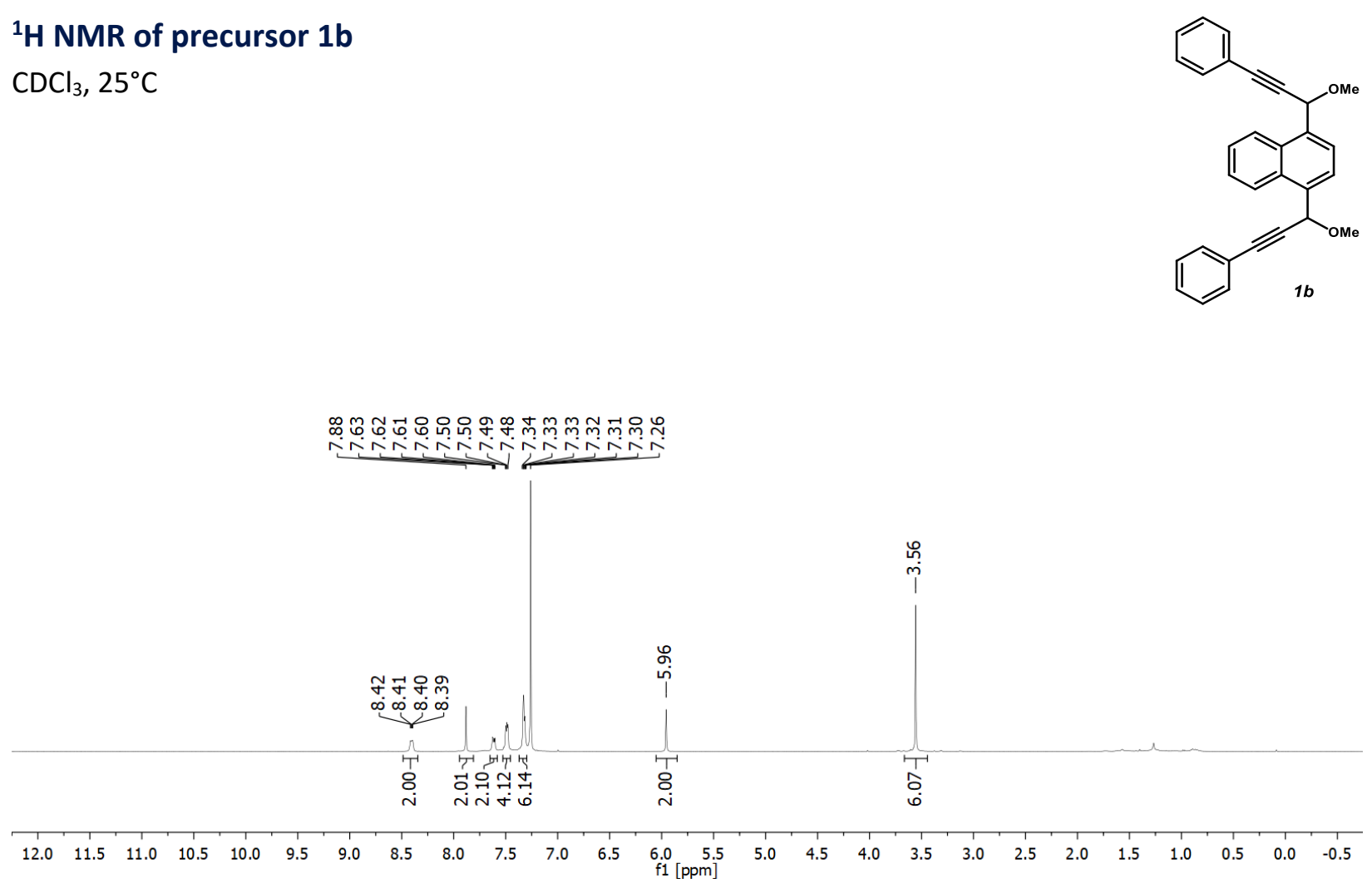

\section{${ }^{13} \mathrm{C}$ NMR of precursor $1 \mathrm{~b}$}

$\mathrm{CDCl}_{3}, 25^{\circ} \mathrm{C}$
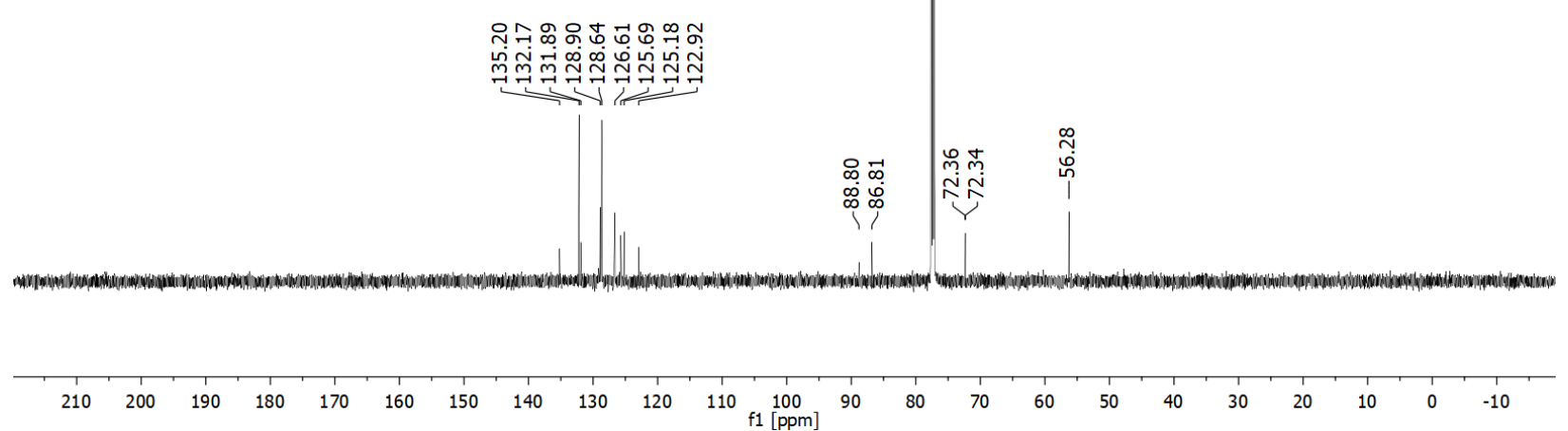


\section{${ }^{1} \mathrm{H}$ NMR of precursor $1 \mathrm{C}$}

$\mathrm{CDCl}_{3}, 25^{\circ} \mathrm{C}$
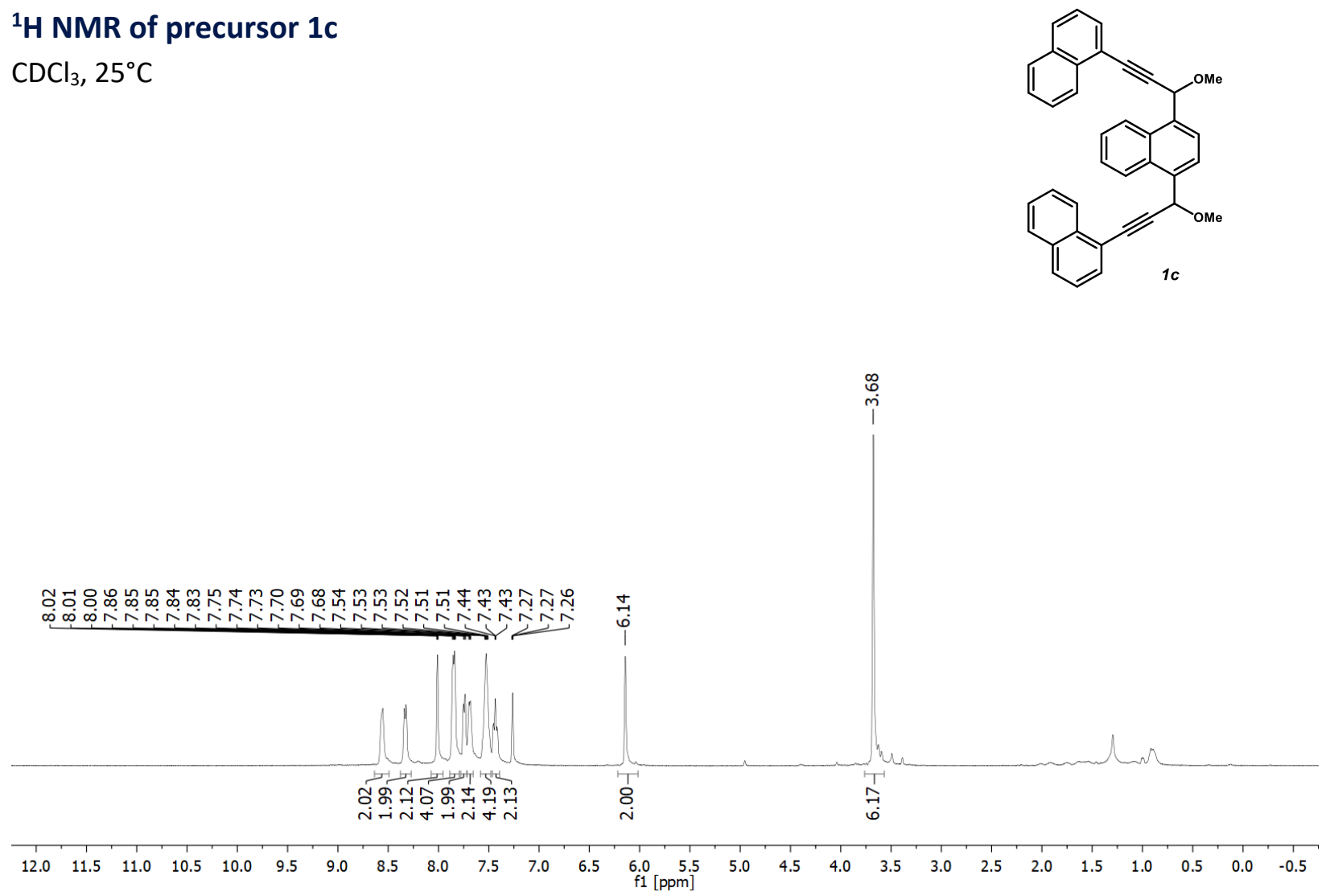

${ }^{13} \mathrm{C}$ NMR of precursor $1 \mathrm{c}$

$\mathrm{CDCl}_{3}, 25^{\circ} \mathrm{C}$
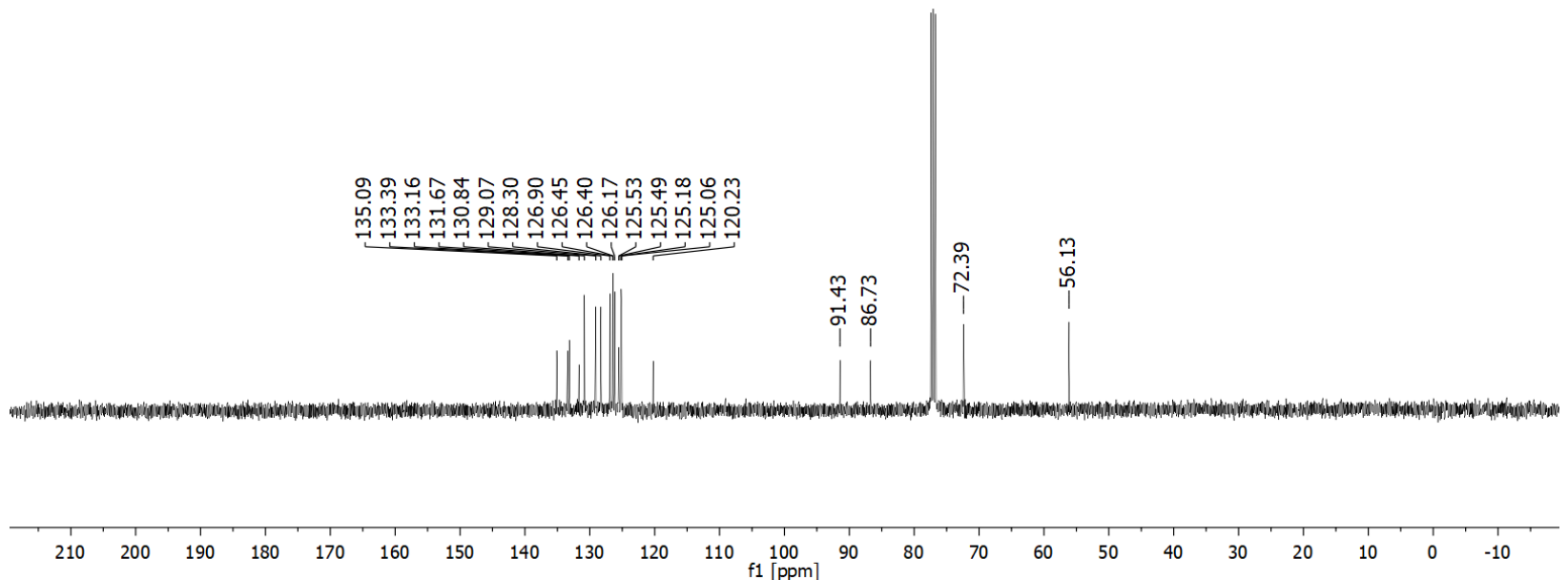
${ }^{1} \mathrm{H}$ NMR of precursor $1 \mathrm{~d}$

$\mathrm{CDCl}_{3}, 25^{\circ} \mathrm{C}$
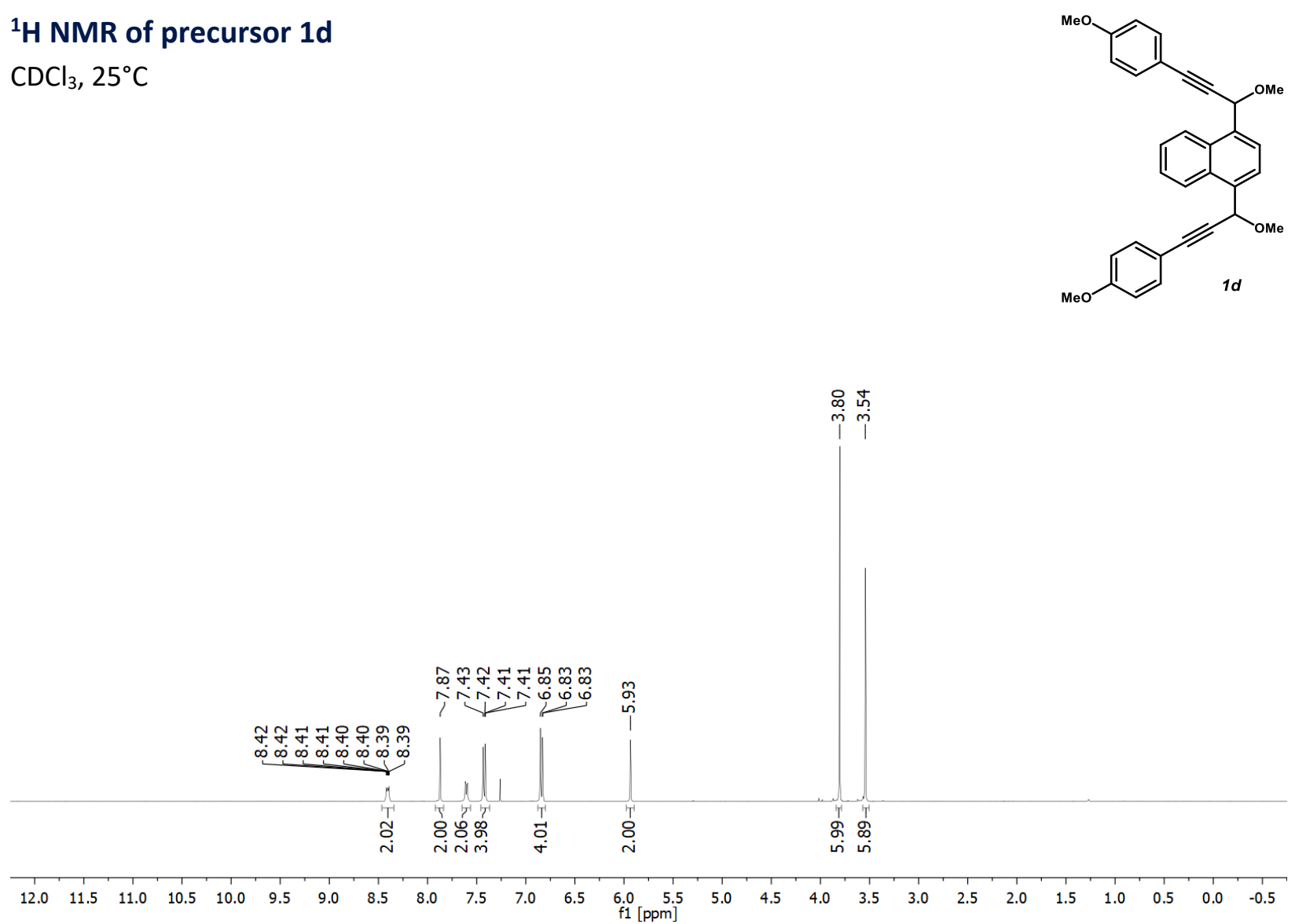

${ }^{13} \mathrm{C}$ NMR of precursor $1 \mathrm{~d}$

$\mathrm{CDCl}_{3}, 25^{\circ} \mathrm{C}$

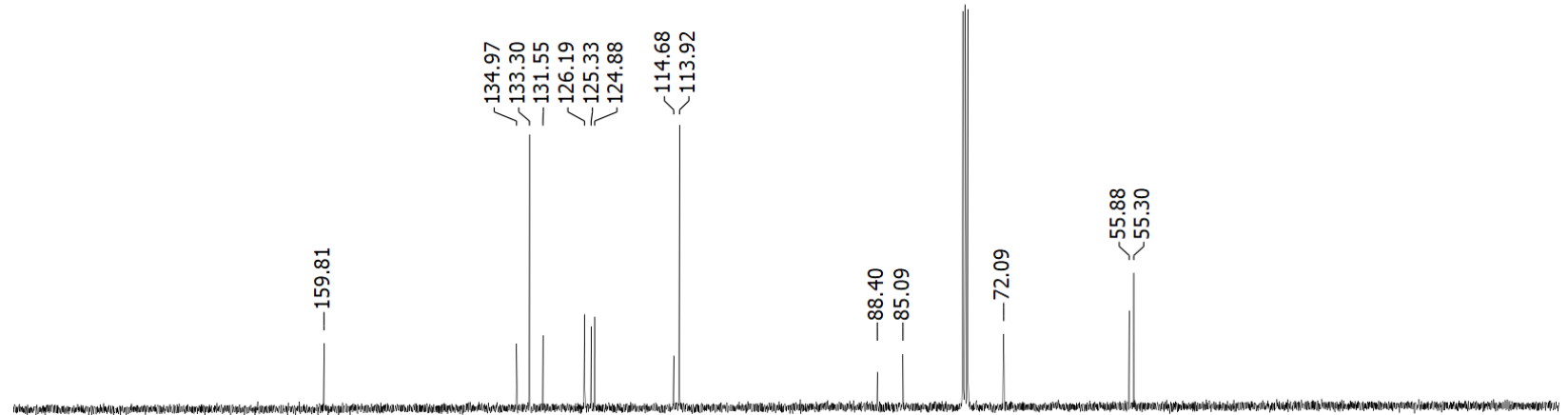




\section{${ }^{1} \mathrm{H}$ NMR of precursor $1 \mathrm{e}$}

$\mathrm{CDCl}_{3}, 25^{\circ} \mathrm{C}$
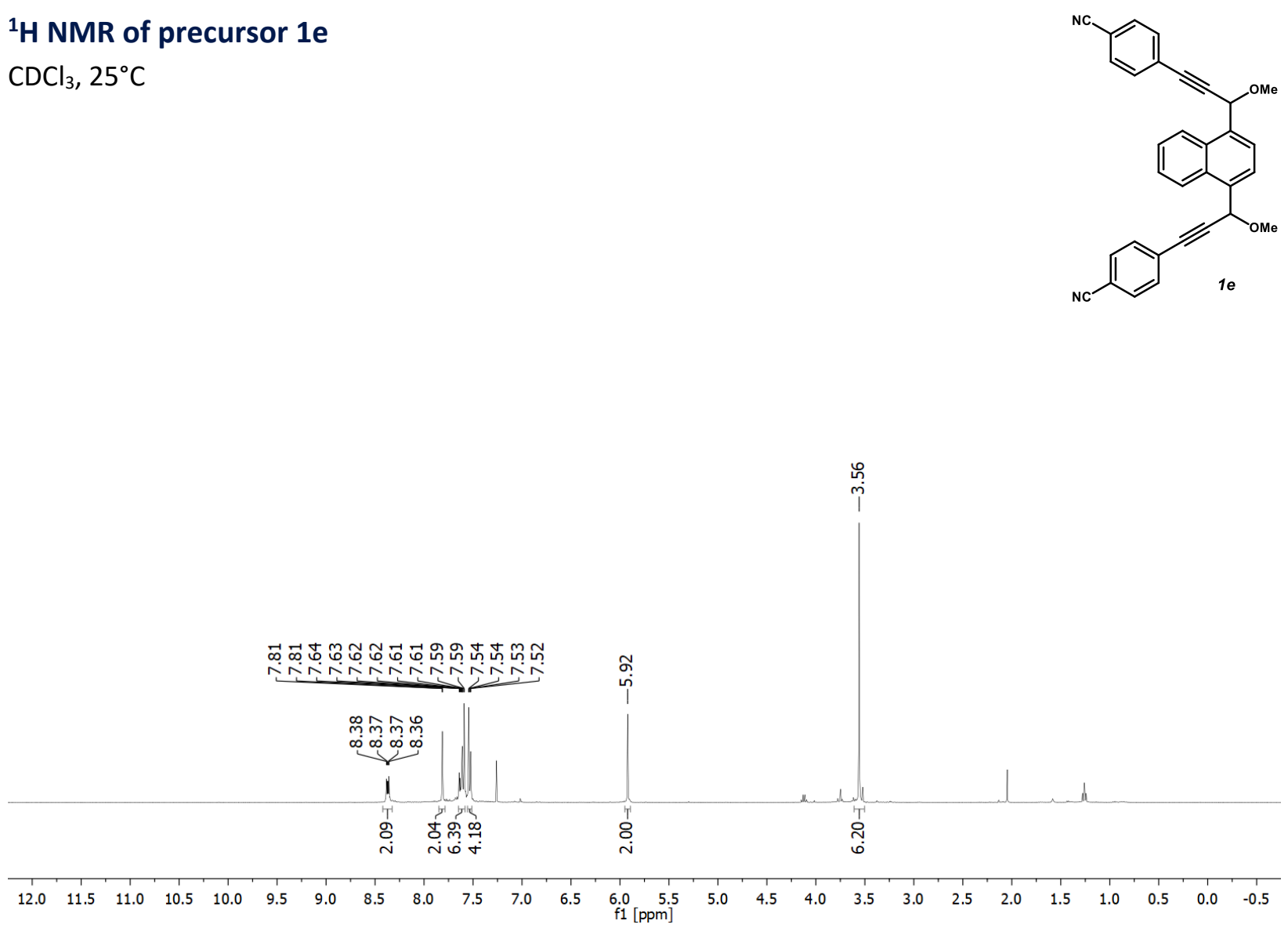

\section{${ }^{13} \mathrm{C}$ NMR of precursor $1 \mathrm{e}$}

$\mathrm{CDCl}_{3}, 25^{\circ} \mathrm{C}$
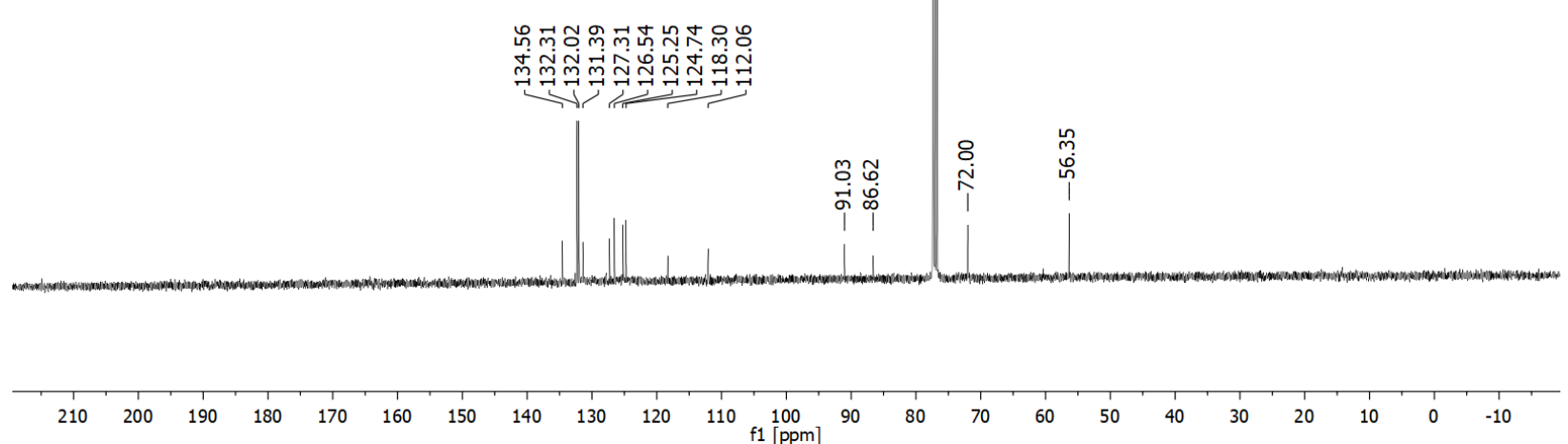
${ }^{1} \mathrm{H}$ NMR of precursor $1 \mathrm{f}$

$\mathrm{CDCl}_{3}, 25^{\circ} \mathrm{C}$
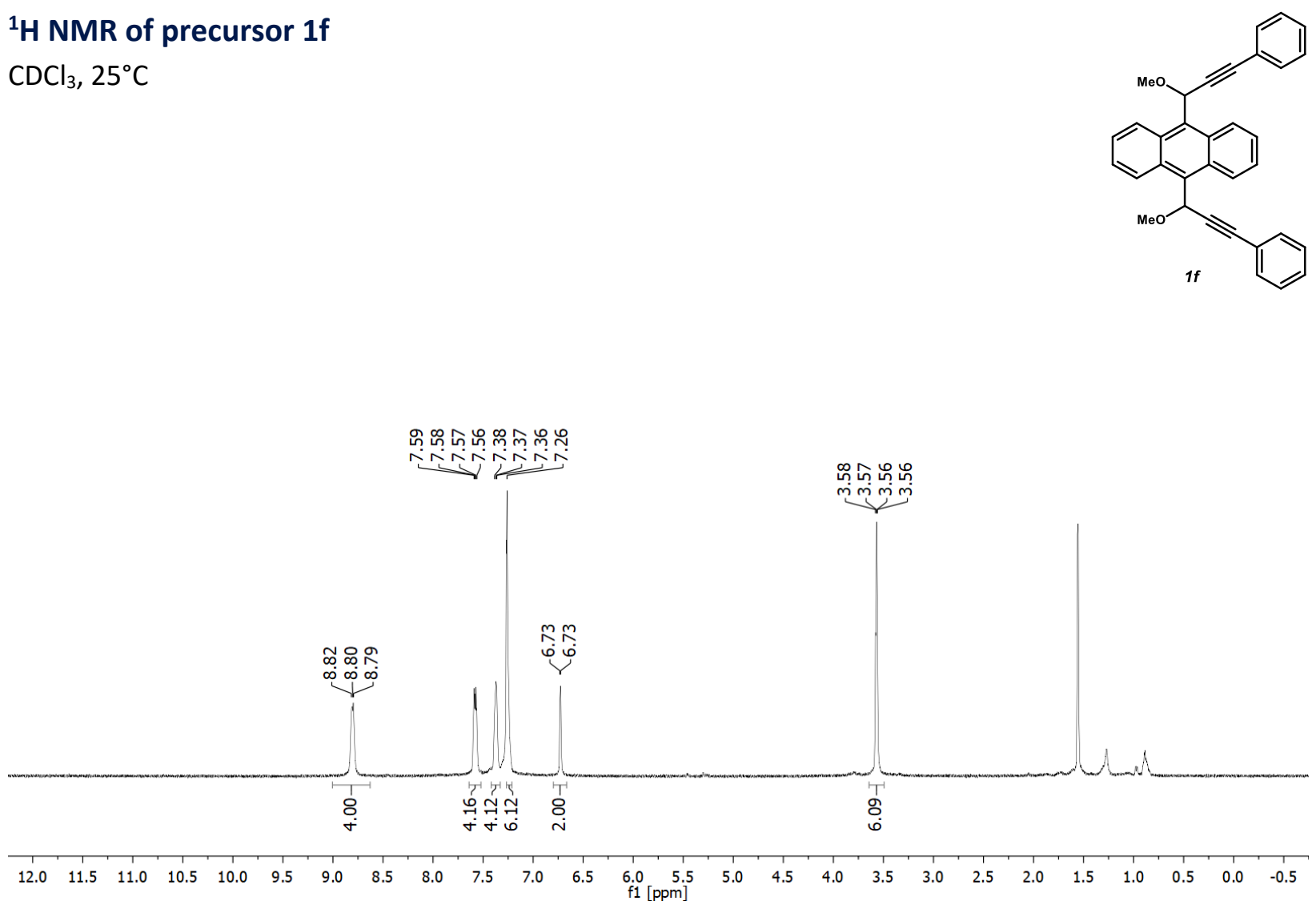

${ }^{13} \mathrm{C}$ NMR of precursor $1 \mathrm{f}$

$\mathrm{CDCl}_{3}, 25^{\circ} \mathrm{C}$

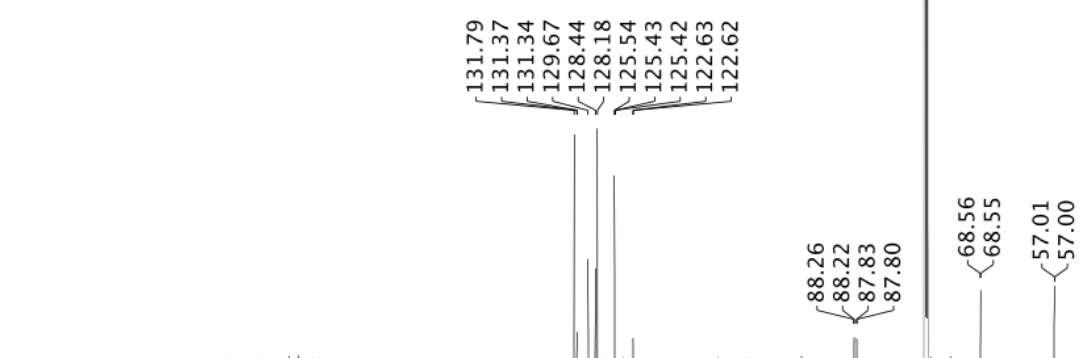

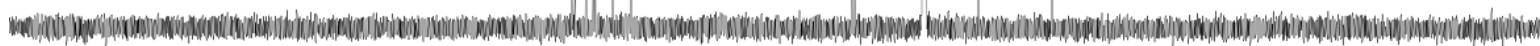

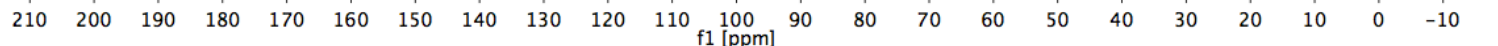


${ }^{1} \mathrm{H}$ NMR of precursor $1 \mathrm{~g}$

$\mathrm{CDCl}_{3}, 25^{\circ} \mathrm{C}$
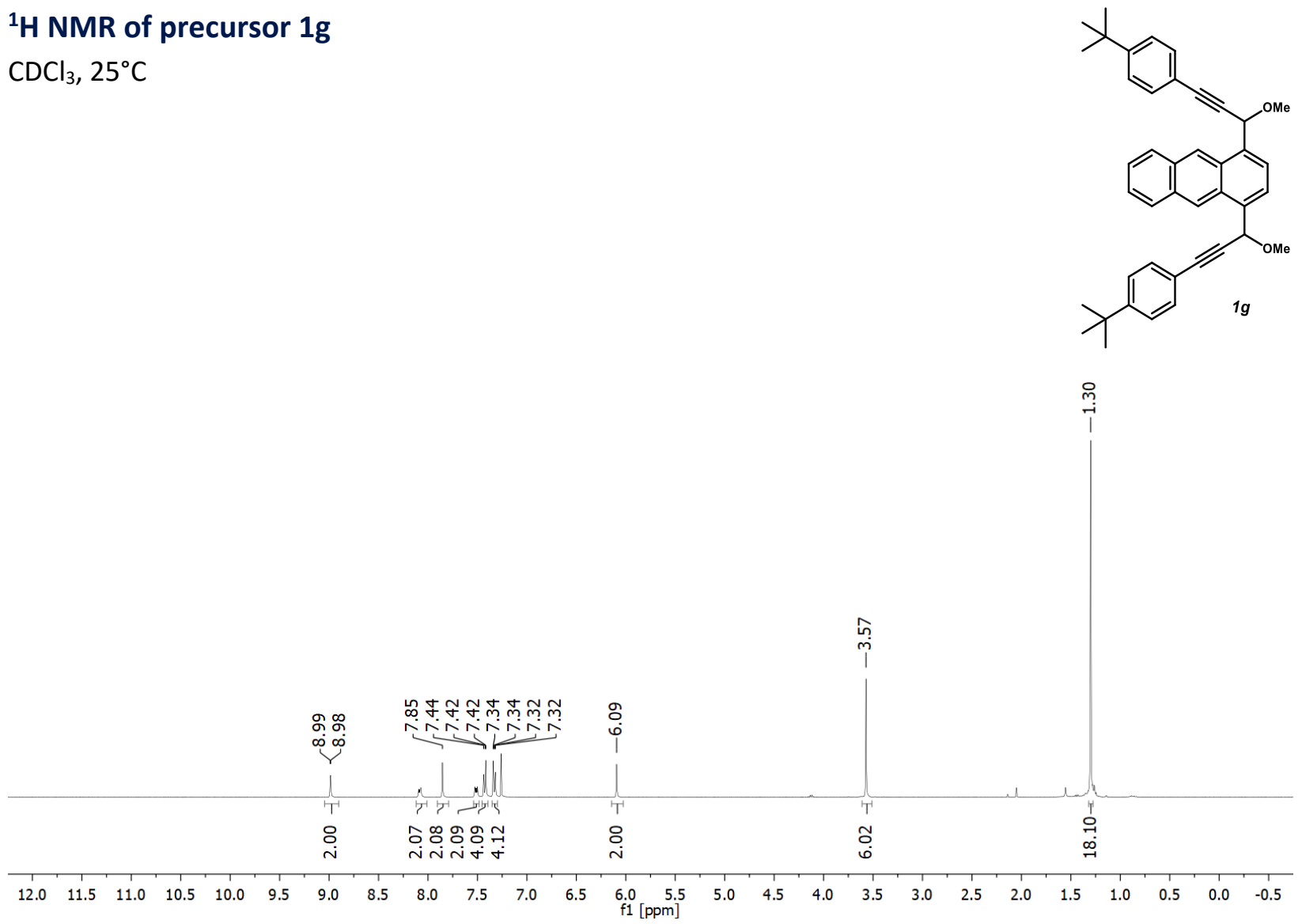

${ }^{13} \mathrm{C}$ NMR of precursor $1 \mathrm{~g}$

$\mathrm{CDCl}_{3}, 25^{\circ} \mathrm{C}$

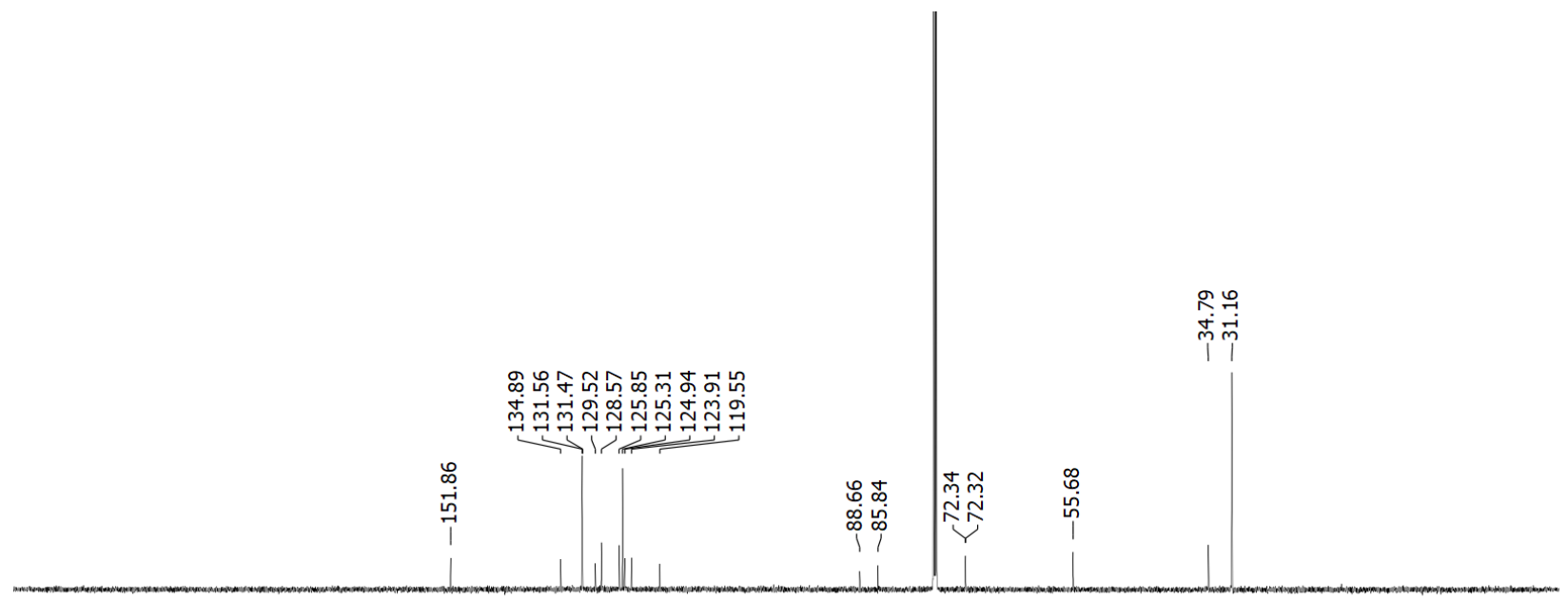

$\begin{array}{lllllllllllllllllllllll}210 & 200 & 190 & 180 & 170 & 160 & 150 & 140 & 130 & 120 & 110 & \begin{array}{l}100 \\ \mathrm{f} 1[\mathrm{ppm}]\end{array} & 90 & 80 & 70 & 60 & 50 & 40 & 30 & 20 & 10 & 0 & -10\end{array}$ 


\section{${ }^{1} \mathrm{H}$ NMR of precursor $\mathbf{1 h}$}

$\mathrm{CDCl}_{3}, 25^{\circ} \mathrm{C}$
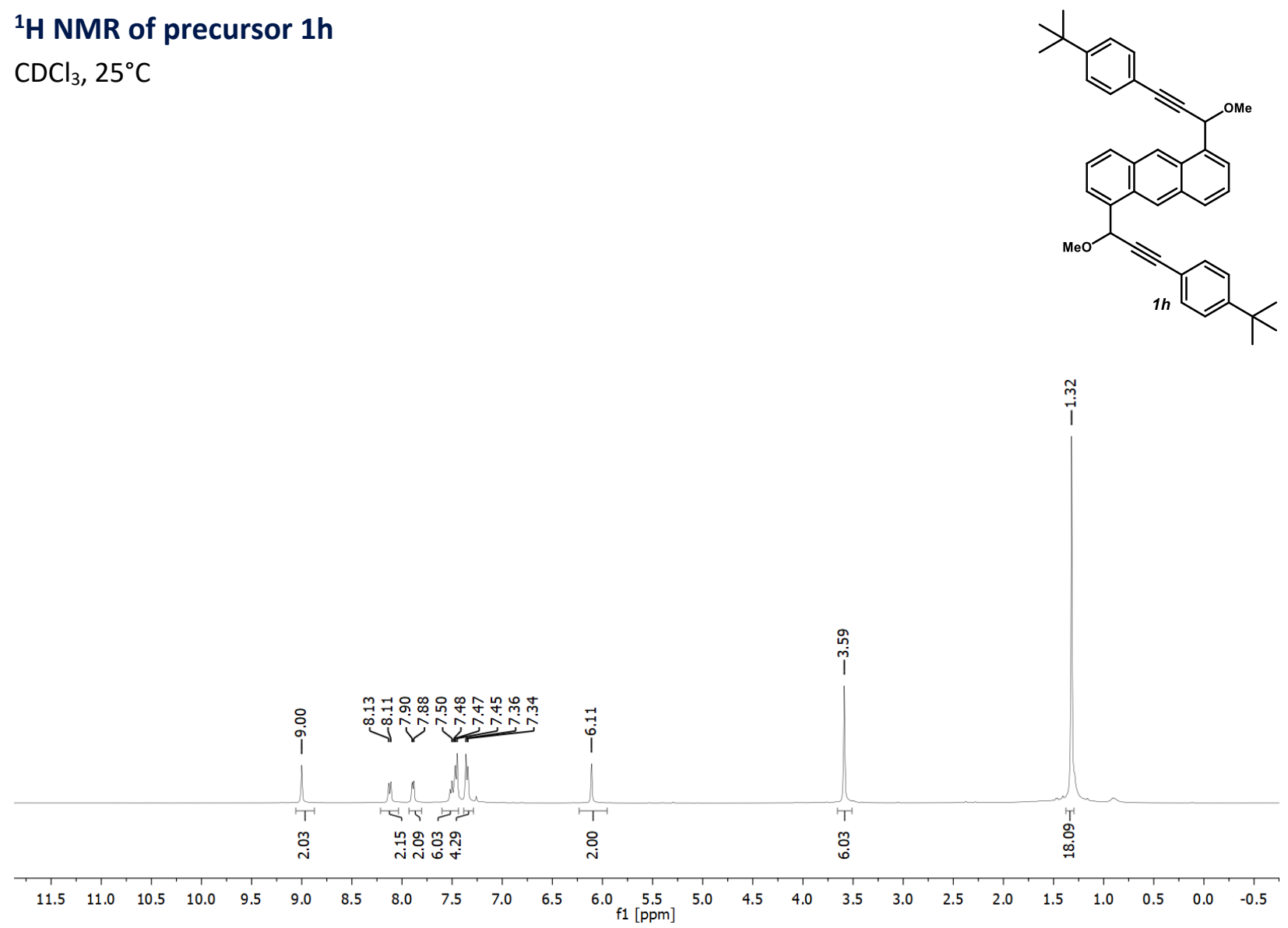

${ }^{13} \mathrm{C}$ NMR of precursor $1 \mathrm{~h}$

$\mathrm{CDCl}_{3}, 25^{\circ} \mathrm{C}$

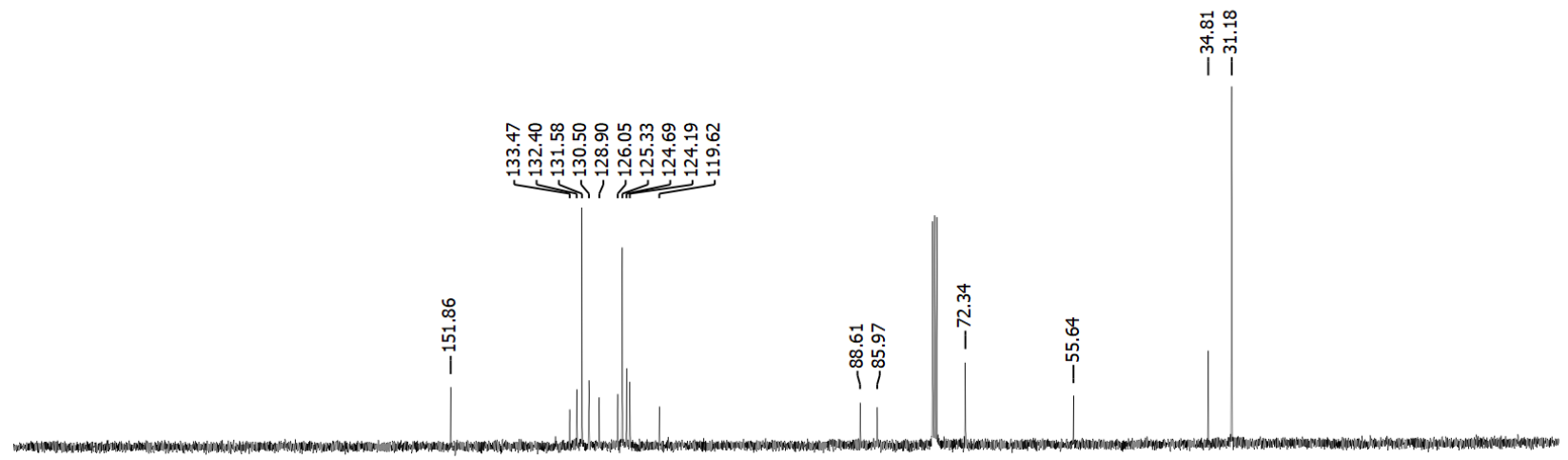
$\begin{array}{llllllllllll}210 & 200 & 190 & 180 & 170 & 160 & 150 & 140 & 130 & 120 & 110 & \begin{array}{l}100 \\ \mathrm{f} 1[\mathrm{ppm}]\end{array}\end{array}$ 
${ }^{1} \mathrm{H}$ NMR of precursor $1 \mathbf{i}$

$\mathrm{CDCl}_{3}, 25^{\circ} \mathrm{C}$
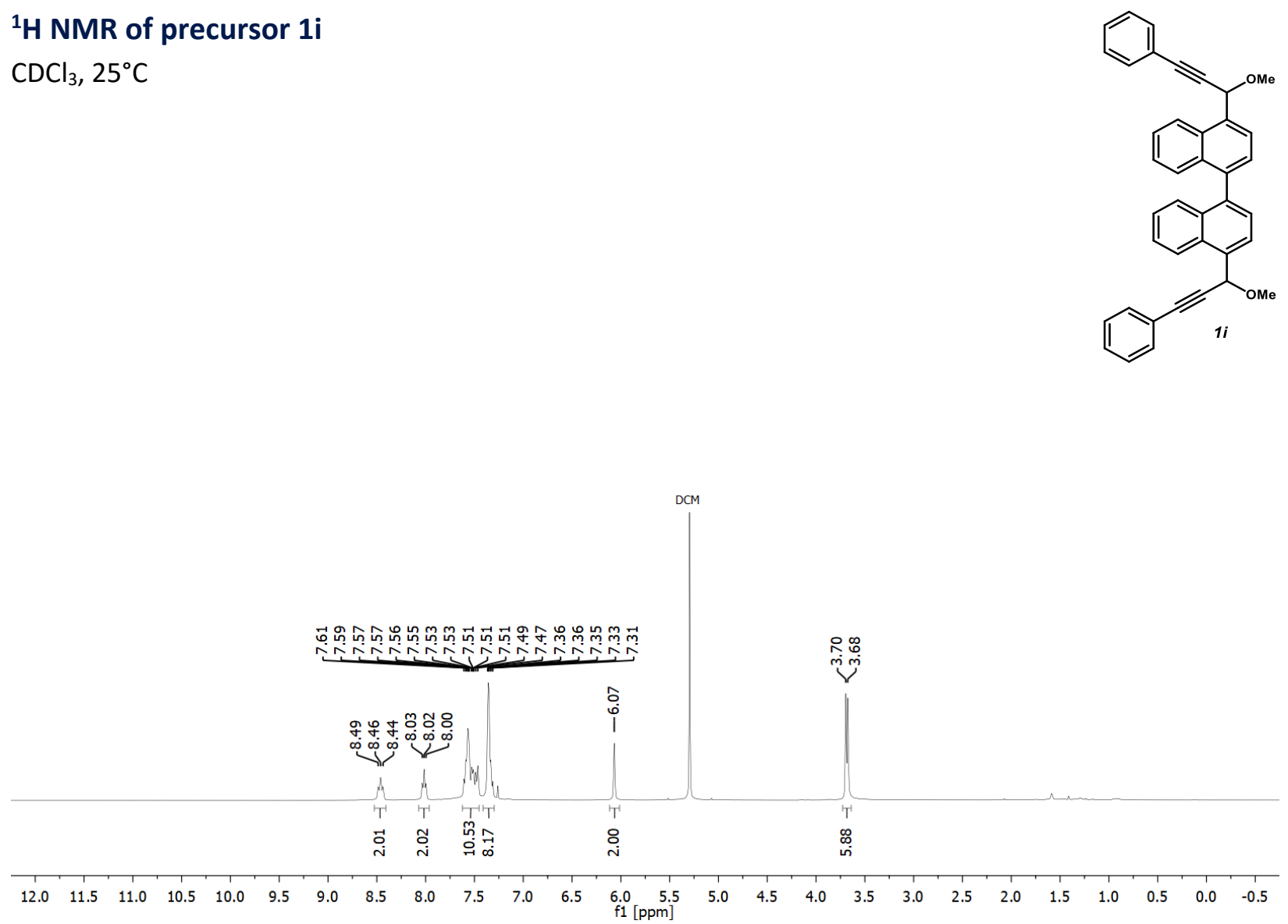

\section{${ }^{13} \mathrm{C}$ NMR of precursor $1 \mathrm{i}$}

$\mathrm{CDCl}_{3}, 25^{\circ} \mathrm{C}$

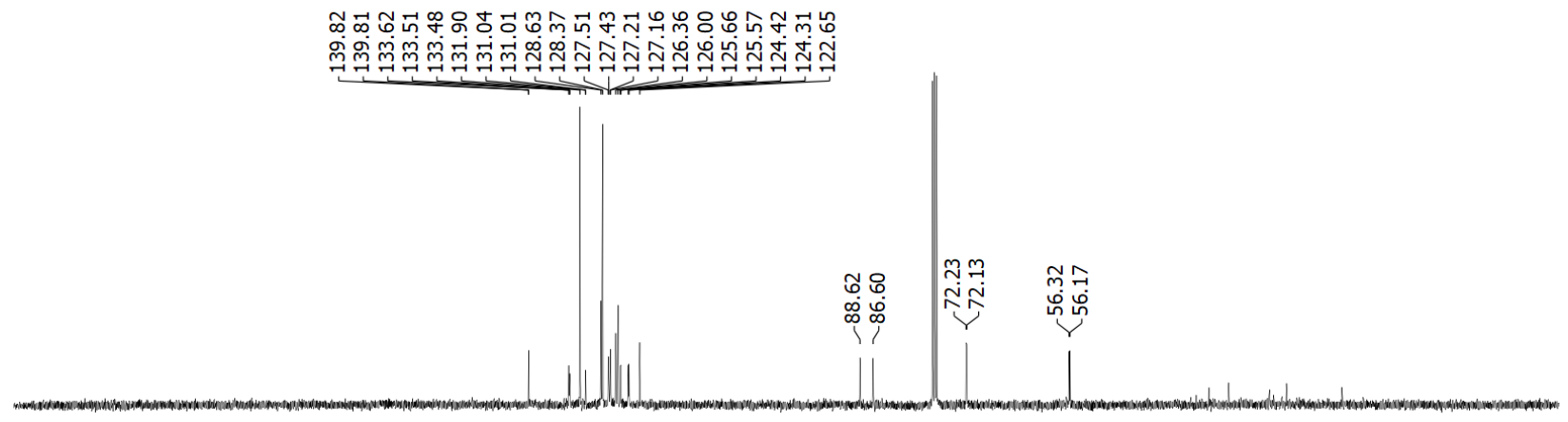

$\begin{array}{lllllllllllllllllllllll}210 & 200 & 190 & 180 & 170 & 160 & 150 & 140 & 130 & 120 & 110 & 100 & 90 & 80 & 70 & 60 & 50 & 40 & 30 & 20 & 10 & 0 & -10\end{array}$ 
${ }^{1} \mathbf{H}$ NMR of precursor $1 \mathbf{j}$

$\mathrm{CDCl}_{3}, 25^{\circ} \mathrm{C}$
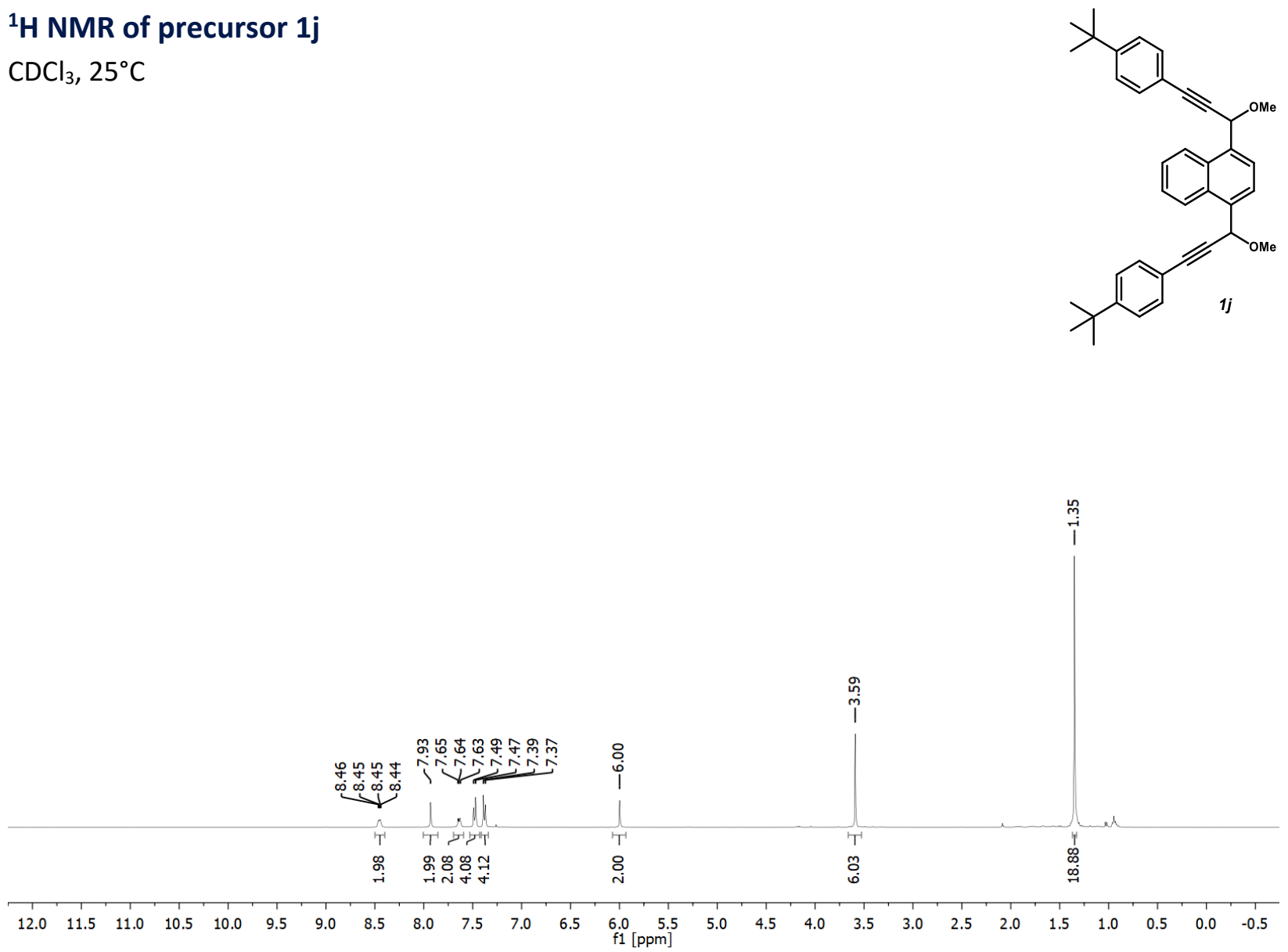

${ }^{13} \mathrm{C}$ NMR of precursor $1 \mathbf{j}$

$\mathrm{CDCl}_{3}, 25^{\circ} \mathrm{C}$

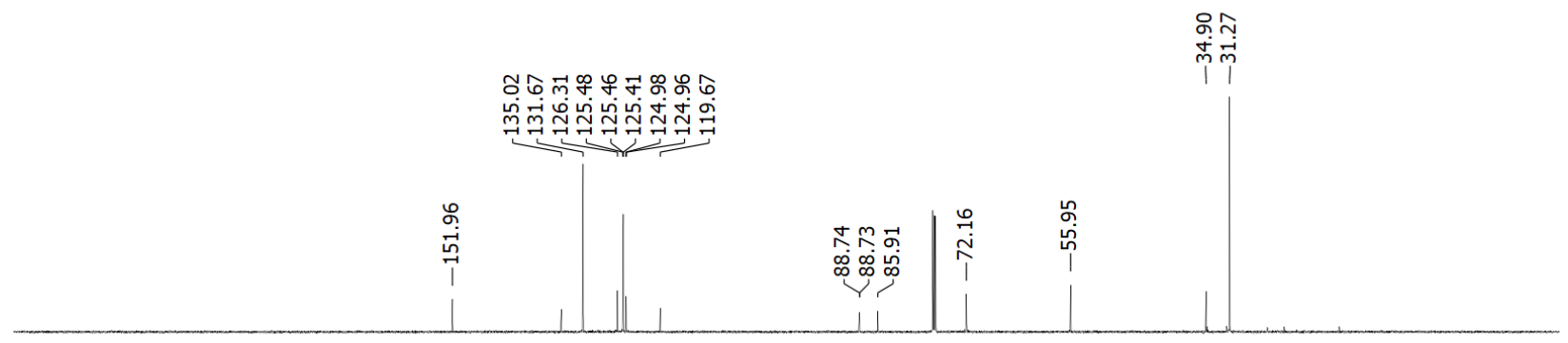

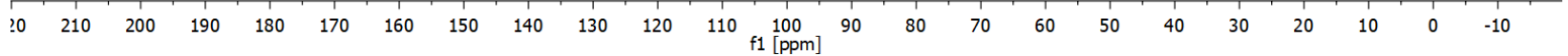


${ }^{1} \mathrm{H}$ NMR of distannylpyrene $2 \mathrm{a}$

$\mathrm{CDCl}_{3}, 25^{\circ} \mathrm{C}$

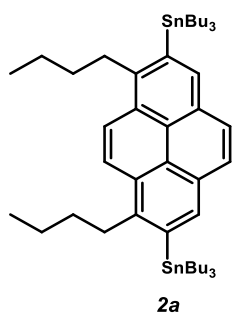

$2 a$

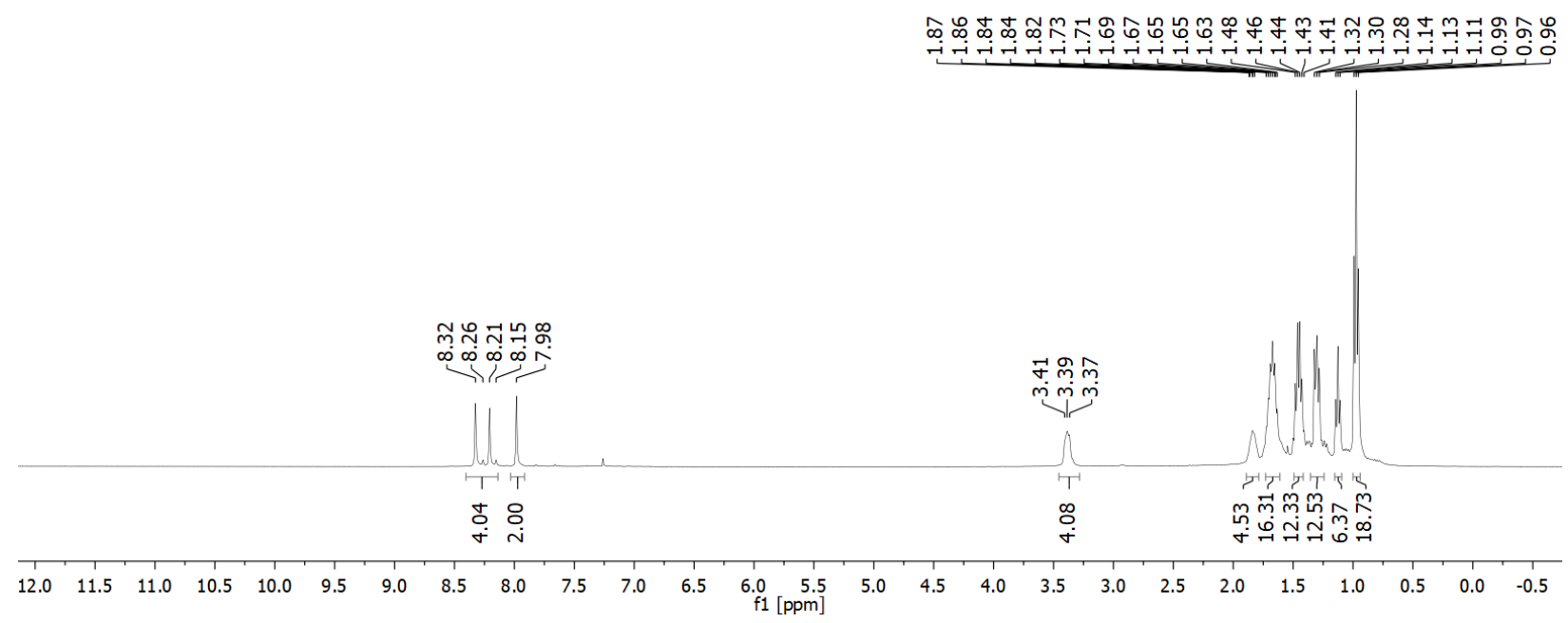

${ }^{13} \mathrm{C}$ NMR of distannylpyrene $2 \mathrm{a}$

$\mathrm{CDCl}_{3}, 25^{\circ} \mathrm{C}$

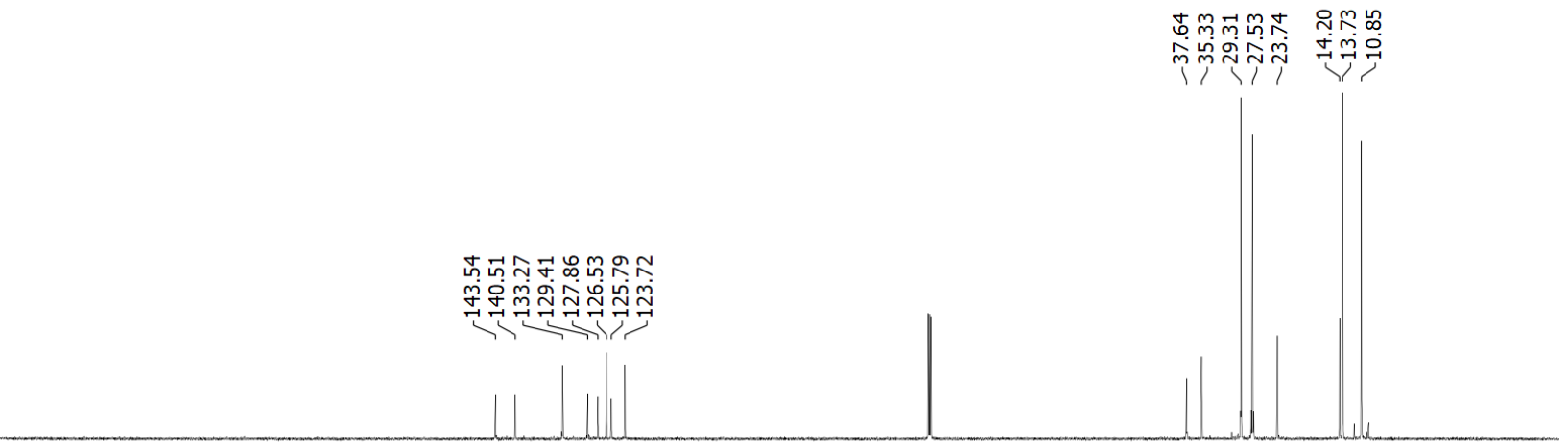

$\begin{array}{lllllllllllllllllllllllllll}210 & 200 & 190 & 180 & 170 & 160 & 150 & 140 & 130 & 120 & 110 & 100 & 90 & 80 & 70 & 60 & 50 & 40 & 30 & 20 & 10 & 0 & -10 & 10\end{array}$ 
${ }^{1} \mathrm{H}$ NMR of distannylpyrene $\mathbf{2 b}$

$\mathrm{CDCl}_{3}, 25^{\circ} \mathrm{C}$

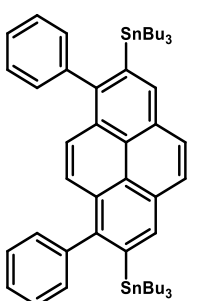

$2 b$

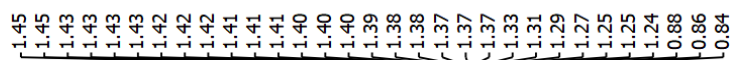

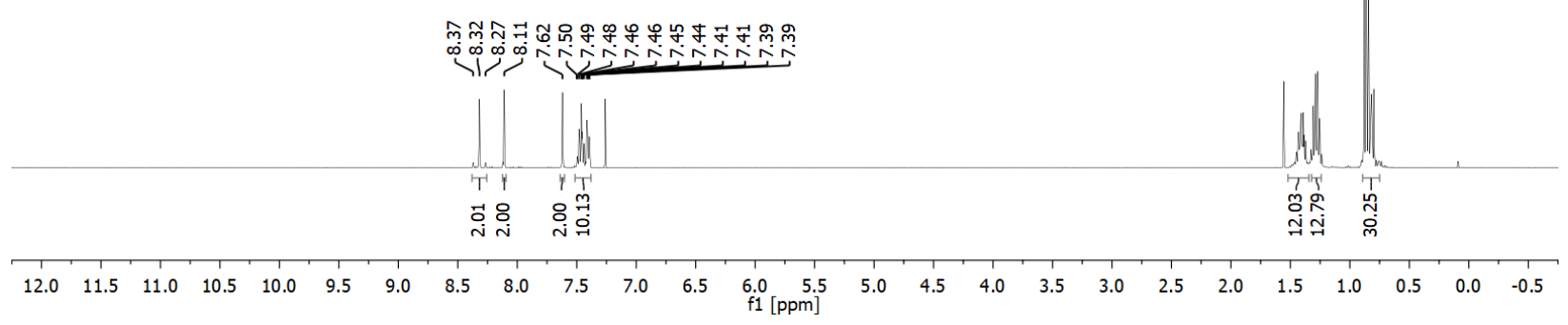

${ }^{13} \mathrm{C}$ NMR of distannylpyrene $2 \mathrm{~b}$

$\mathrm{CDCl}_{3}, 25^{\circ} \mathrm{C}$
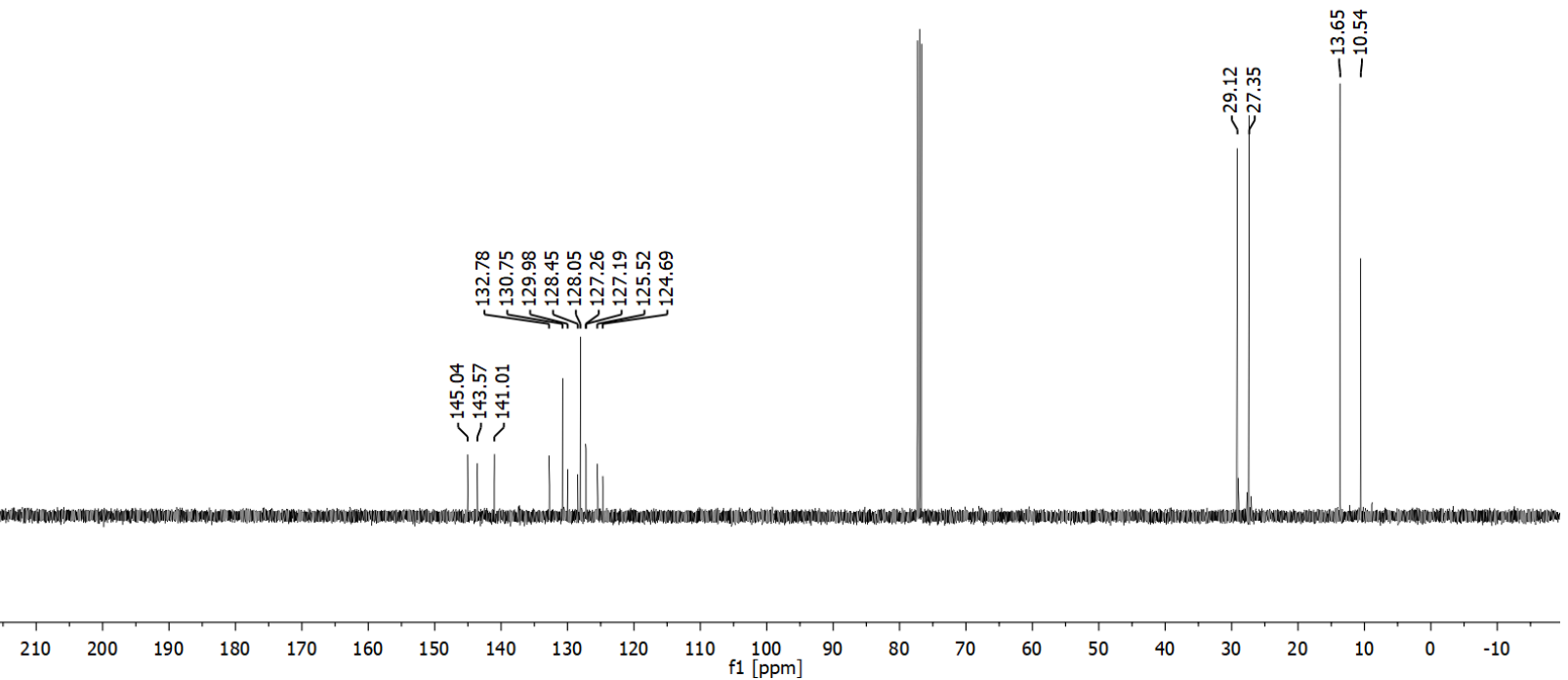
${ }^{1} \mathrm{H}$ NMR of distannylpyrene $2 \mathrm{C}$

$\mathrm{CDCl}_{3}, 25^{\circ} \mathrm{C}$

(mixture of atropisomers)

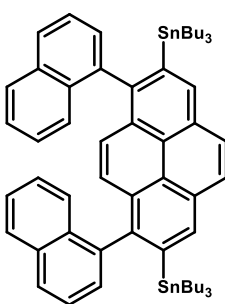

$2 c$

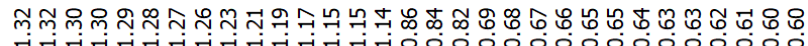

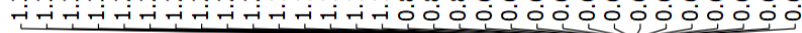

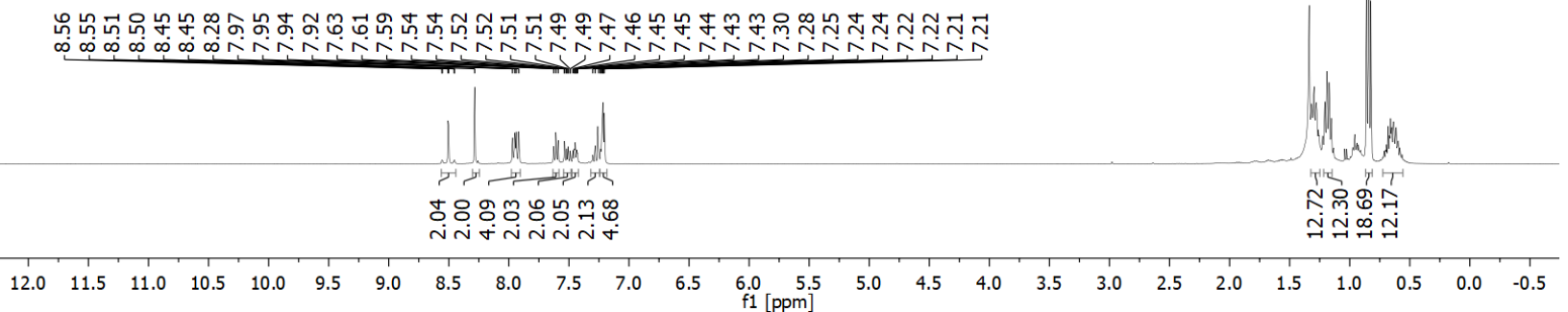

\section{${ }^{13} \mathrm{C}$ NMR of distannylpyrene $2 \mathrm{c}$}

$\mathrm{CDCl}_{3}, 25^{\circ} \mathrm{C}$

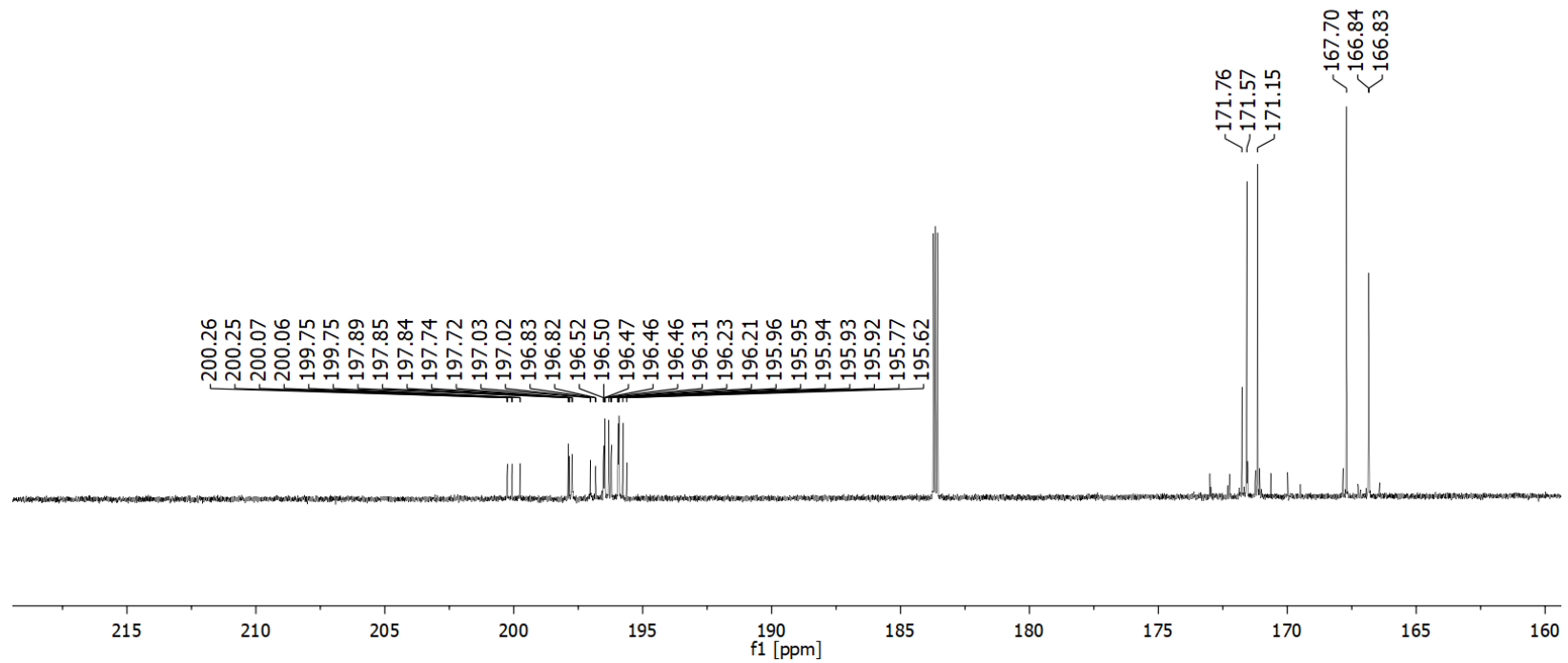


${ }^{1} \mathrm{H}$ NMR of distannylpyrene $\mathbf{2 d}$

$\mathrm{CDCl}_{3}, 25^{\circ} \mathrm{C}$

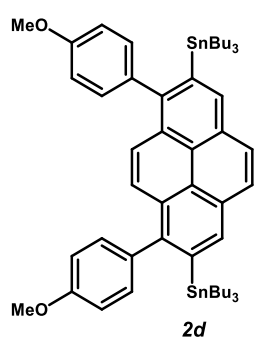

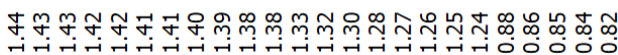

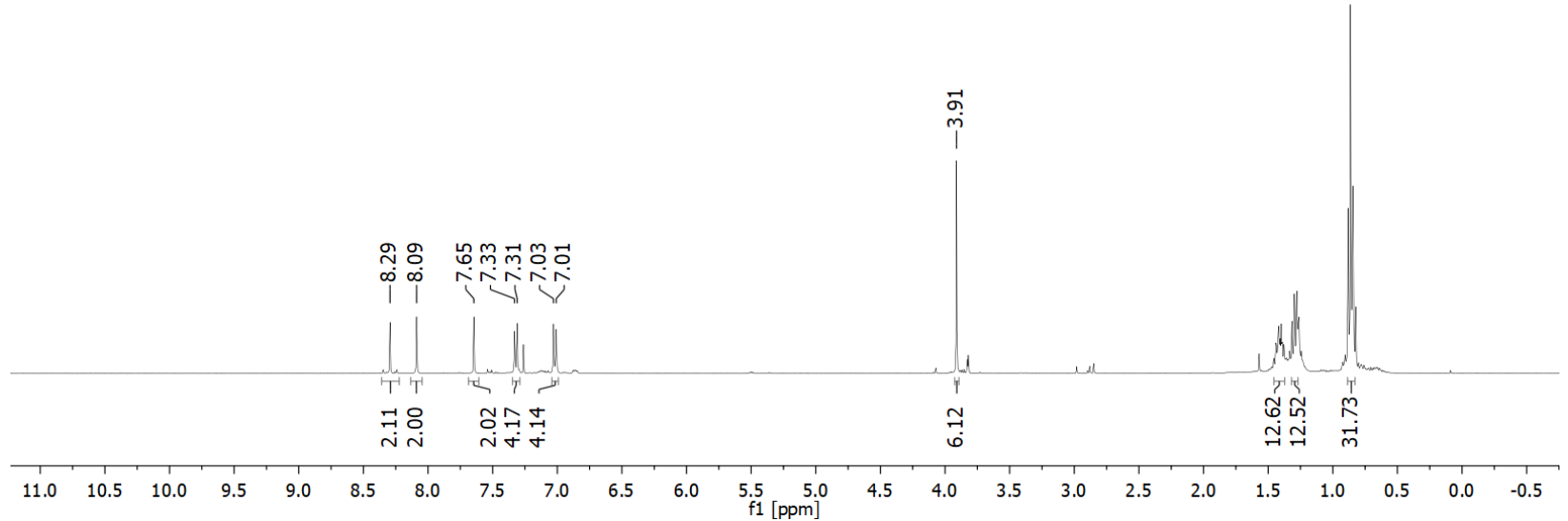

${ }^{13} \mathrm{C}$ NMR of distannylpyrene $2 \mathrm{~d}$

$\mathrm{CDCl}_{3}, 25^{\circ} \mathrm{C}$

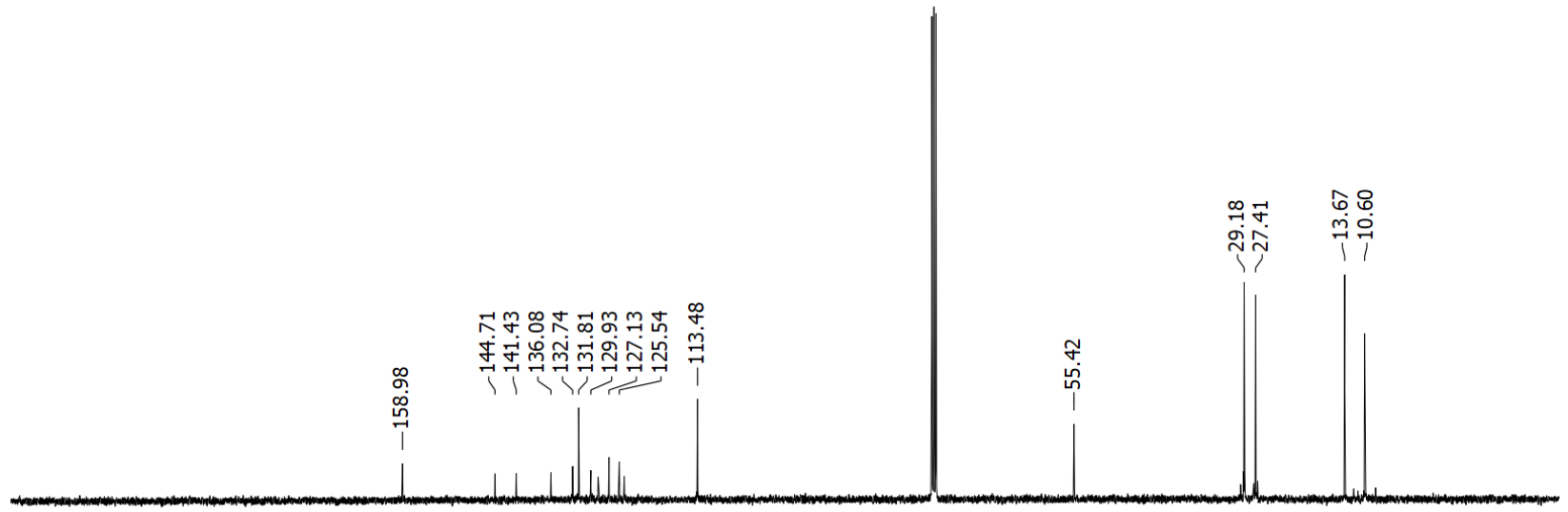

$\begin{array}{llllllllllllllllllllllllllll}210 & 200 & 190 & 180 & 170 & 160 & 150 & 140 & 130 & 120 & 110 & 100 & 90 & 80 & 70 & 60 & 50 & 40 & 30 & 20 & 10 & 0 & -10\end{array}$ 
${ }^{1} \mathrm{H}$ NMR of distannylpyrene $\mathbf{2 e}$

$\mathrm{CDCl}_{3}, 25^{\circ} \mathrm{C}$
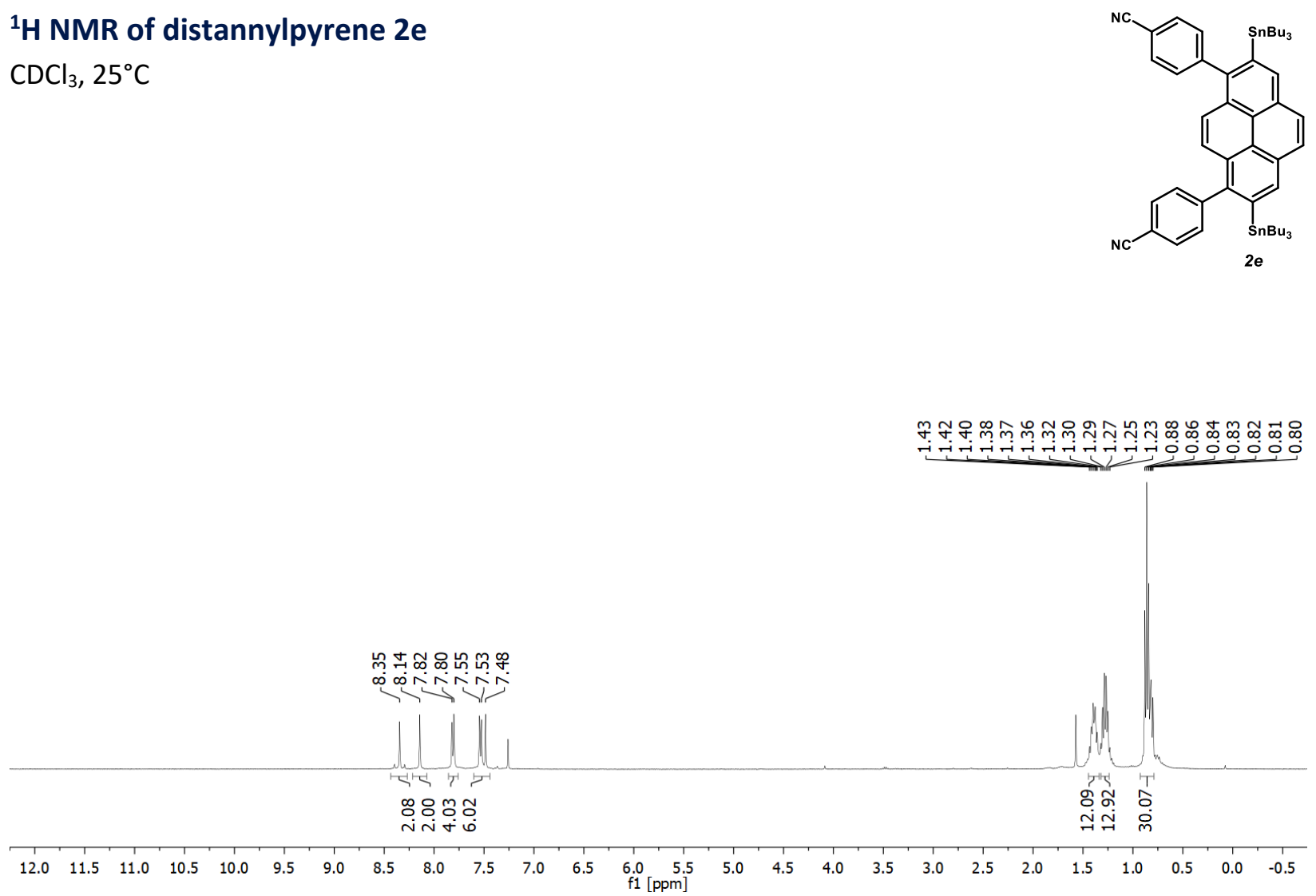

\section{${ }^{13} \mathrm{C}$ NMR of distannylpyrene $2 \mathrm{e}$}

$\mathrm{CDCl}_{3}, 25^{\circ} \mathrm{C}$

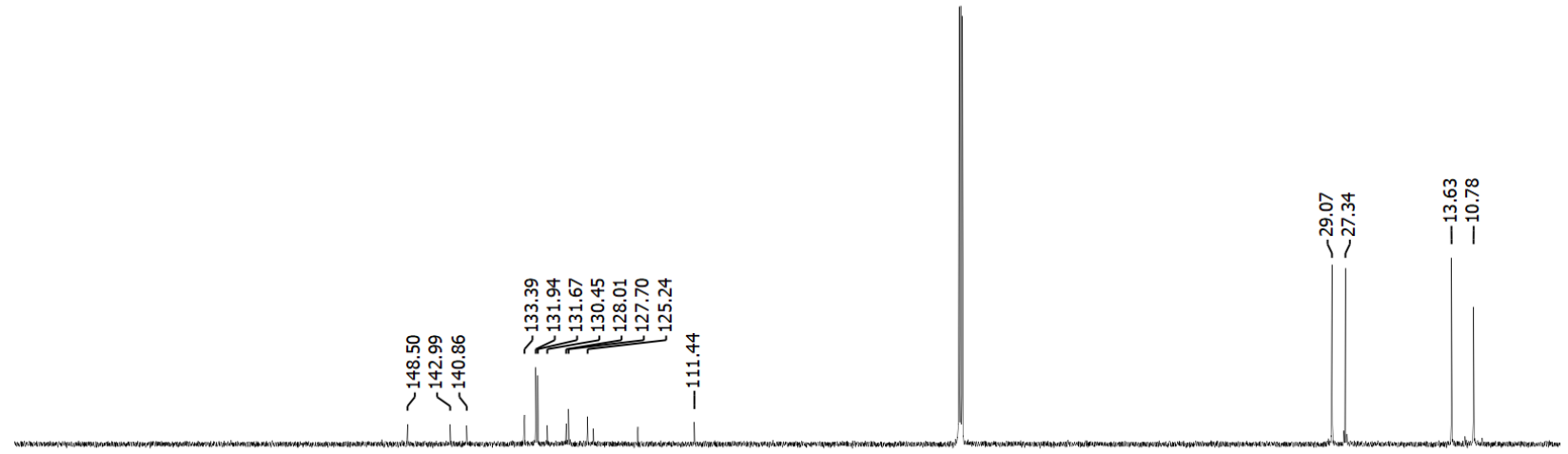

$\begin{array}{llllllllllllllllllllll}190 & 180 & 170 & 160 & 150 & 140 & 130 & 120 & 110 & 100 & 90 & 80 & 70 & 60 & 50 & 40 & 30 & 20 & 10 & 0\end{array}$ 
${ }^{1} \mathrm{H}$ NMR of distannylbenzo[e]pyrene $2 \mathrm{~g}$

$\mathrm{CDCl}_{3}, 25^{\circ} \mathrm{C}$
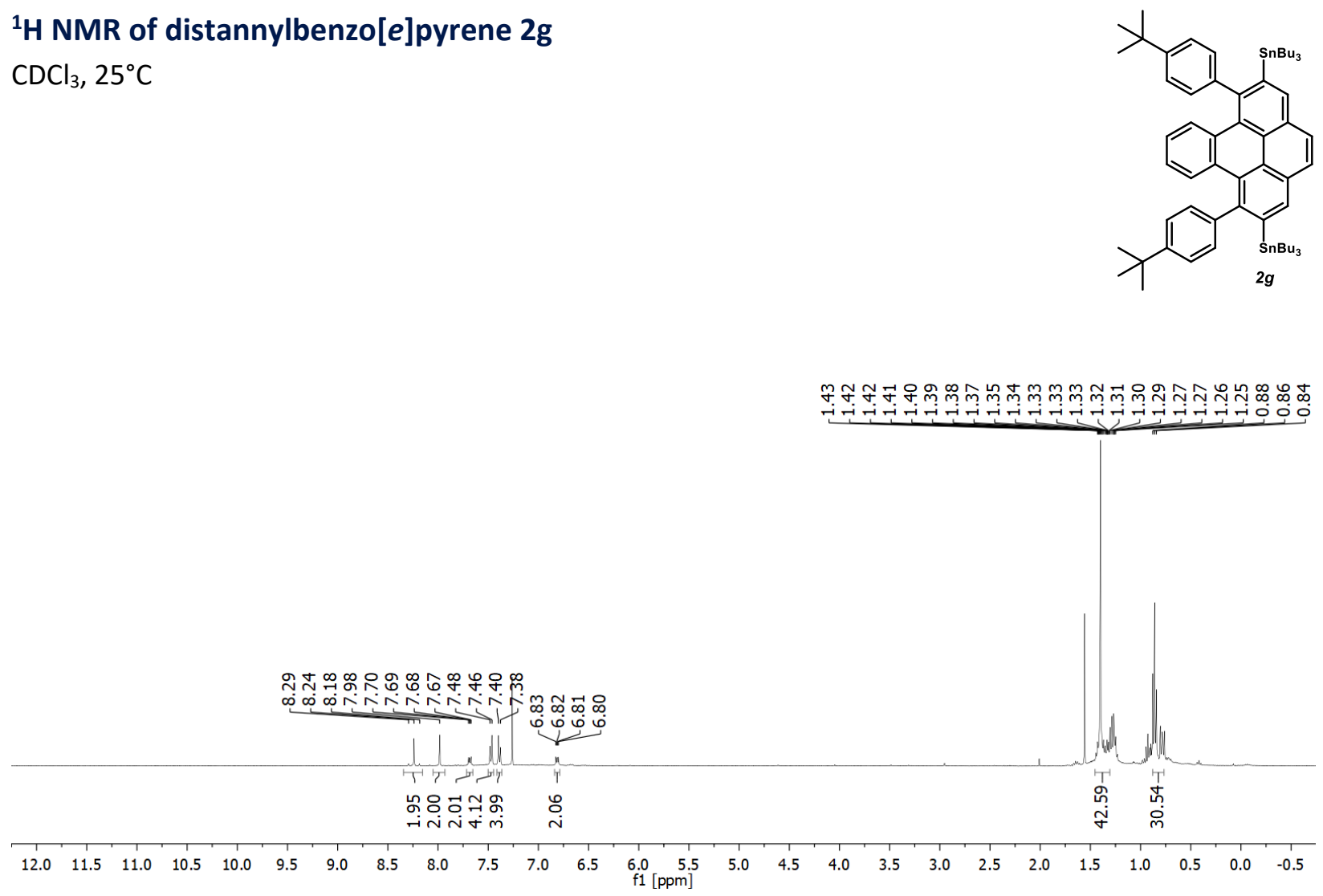

${ }^{13} \mathrm{C}$ NMR of distannylbenzopyrene $2 \mathrm{~g}$

$\mathrm{CDCl}_{3}, 25^{\circ} \mathrm{C}$

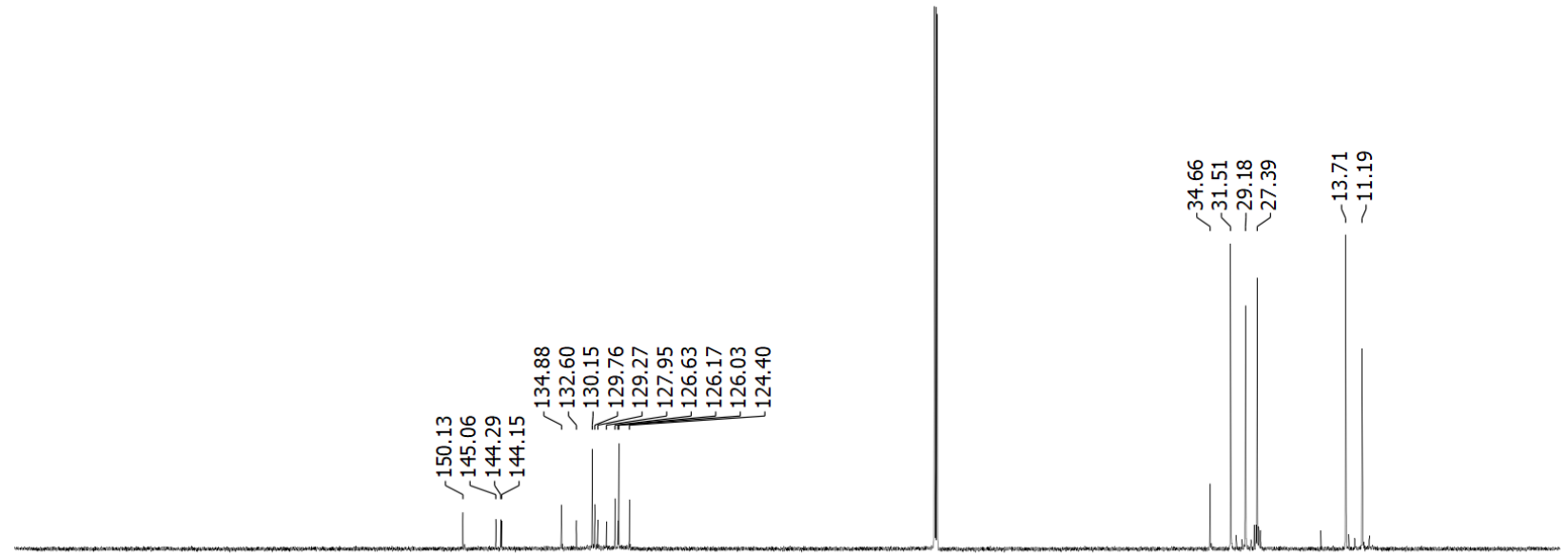

210

$190 \quad 180$

160

150

130

$110 \underset{f 1}{100} 9$ 
${ }^{1} \mathrm{H}$ NMR of distannylperylene $\mathbf{2 h}$

$\mathrm{CDCl}_{3}, 25^{\circ} \mathrm{C}$
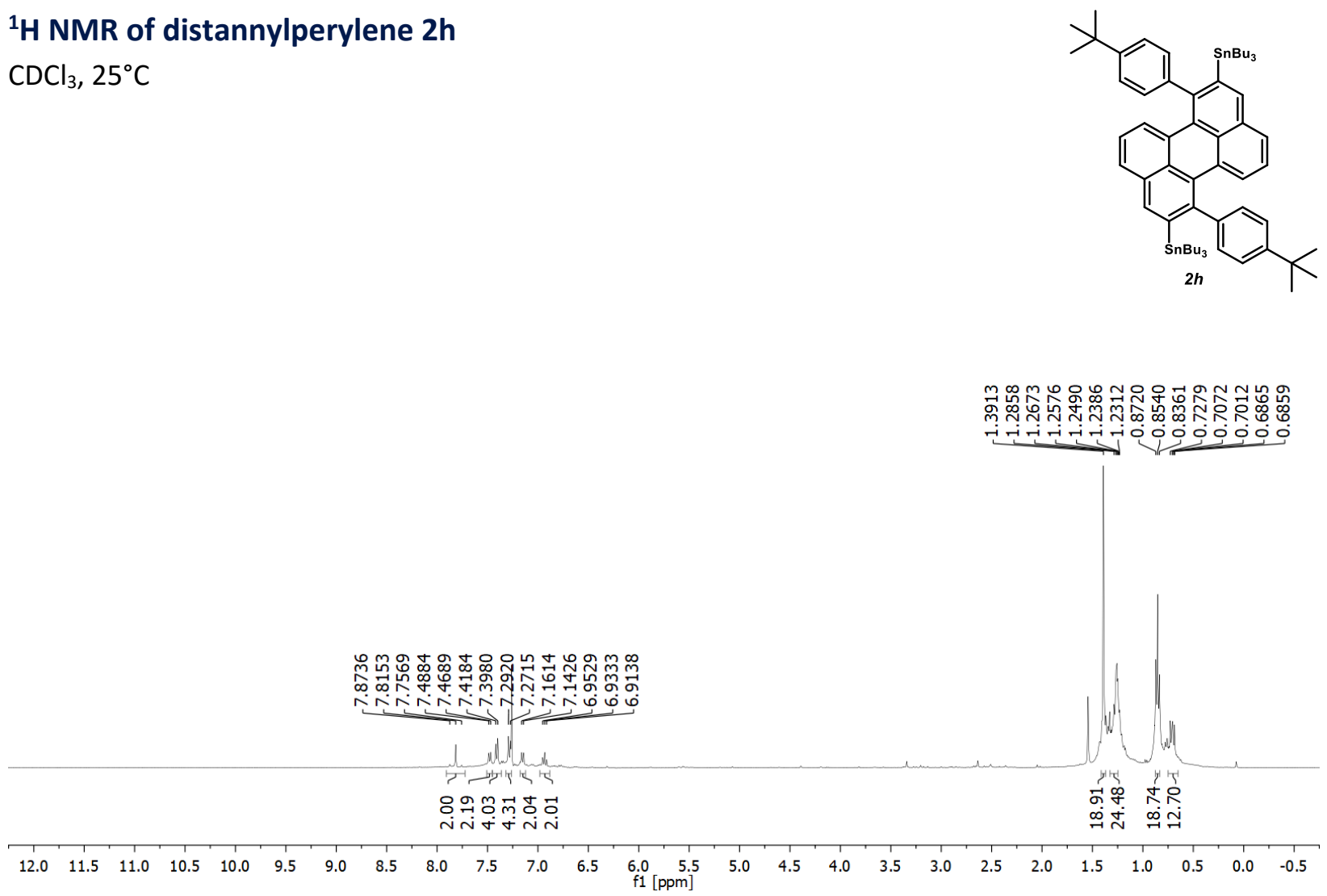

${ }^{13} \mathrm{C}$ NMR of distannylperylene $2 \mathrm{~h}$

$\mathrm{CDCl}_{3}, 25^{\circ} \mathrm{C}$
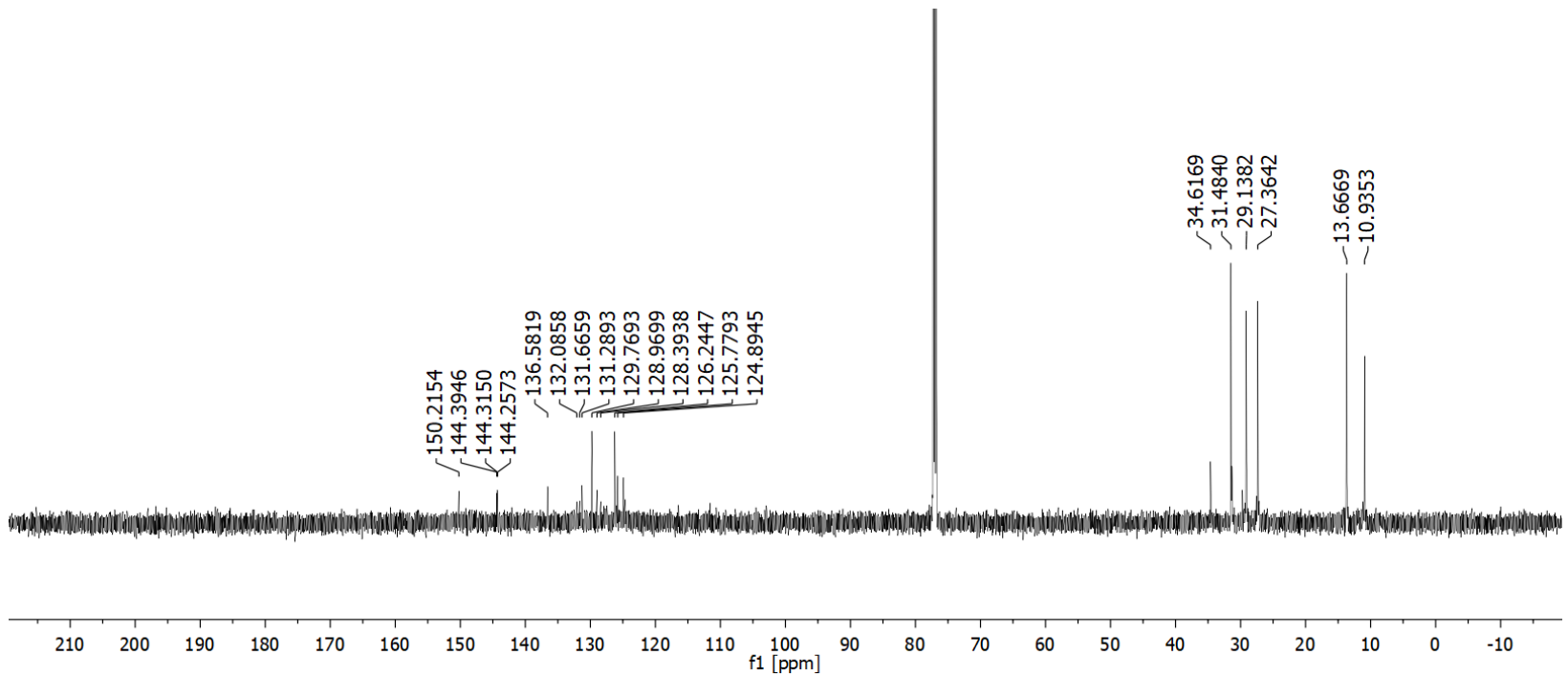


\section{${ }^{1} \mathrm{H}$ NMR of distannylperopyrene $2 \mathrm{i}$}

$\mathrm{CDCl}_{3}, 25^{\circ} \mathrm{C}$
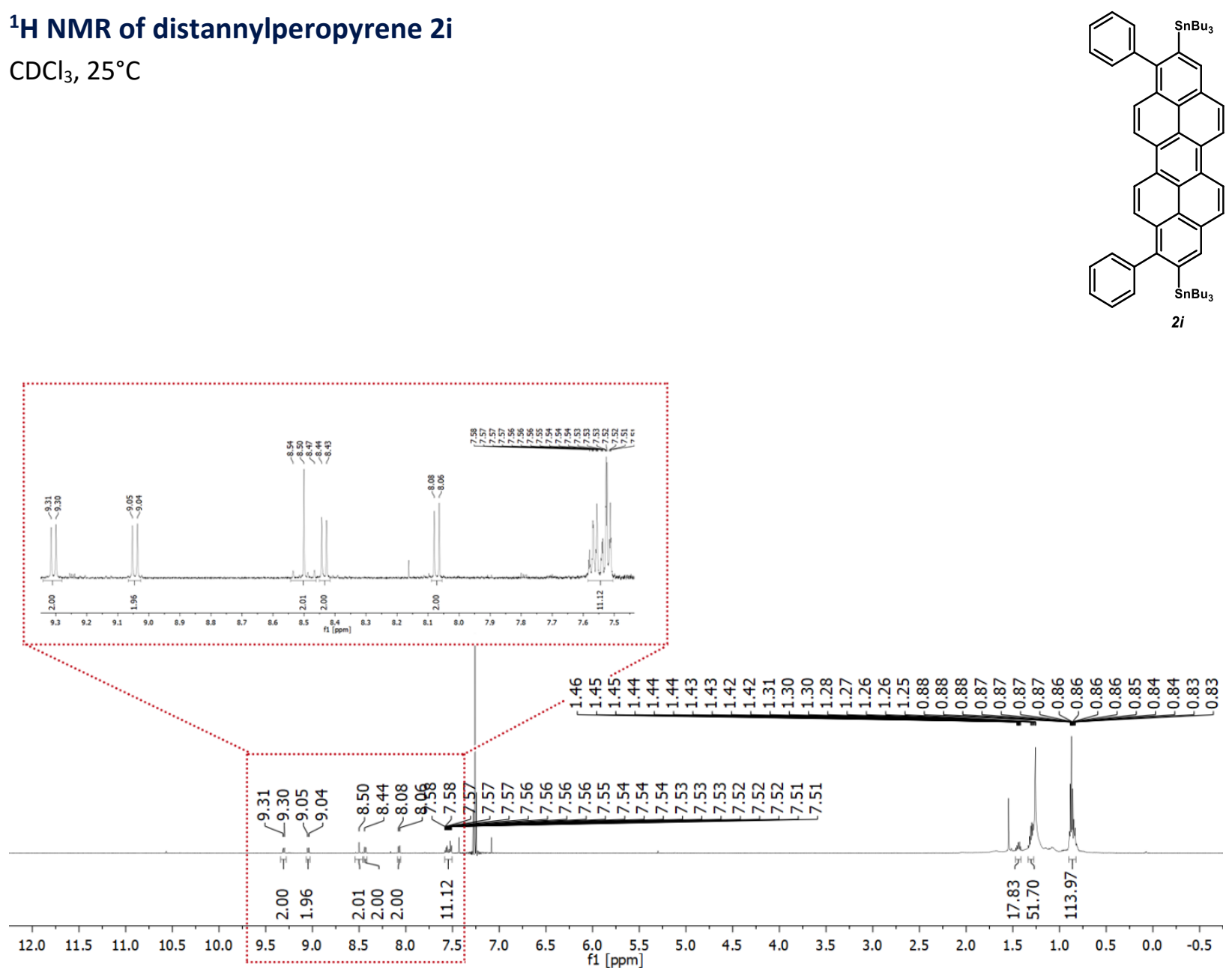

\section{${ }^{13} \mathrm{C}$ NMR of distannylperopyrene $2 \mathbf{i}$}

Not acquired due to low quantitates of pure compound isolated without considerable decomposition. Instead, utilized crude mixture for derivatization reactions (see $\mathbf{3 i}$ and $\mathbf{4 i}$ ). 
${ }^{1} \mathrm{H}$ NMR of diiodopyrene $3 \mathrm{a}$

$\mathrm{CDCl}_{3}, 25^{\circ} \mathrm{C}$
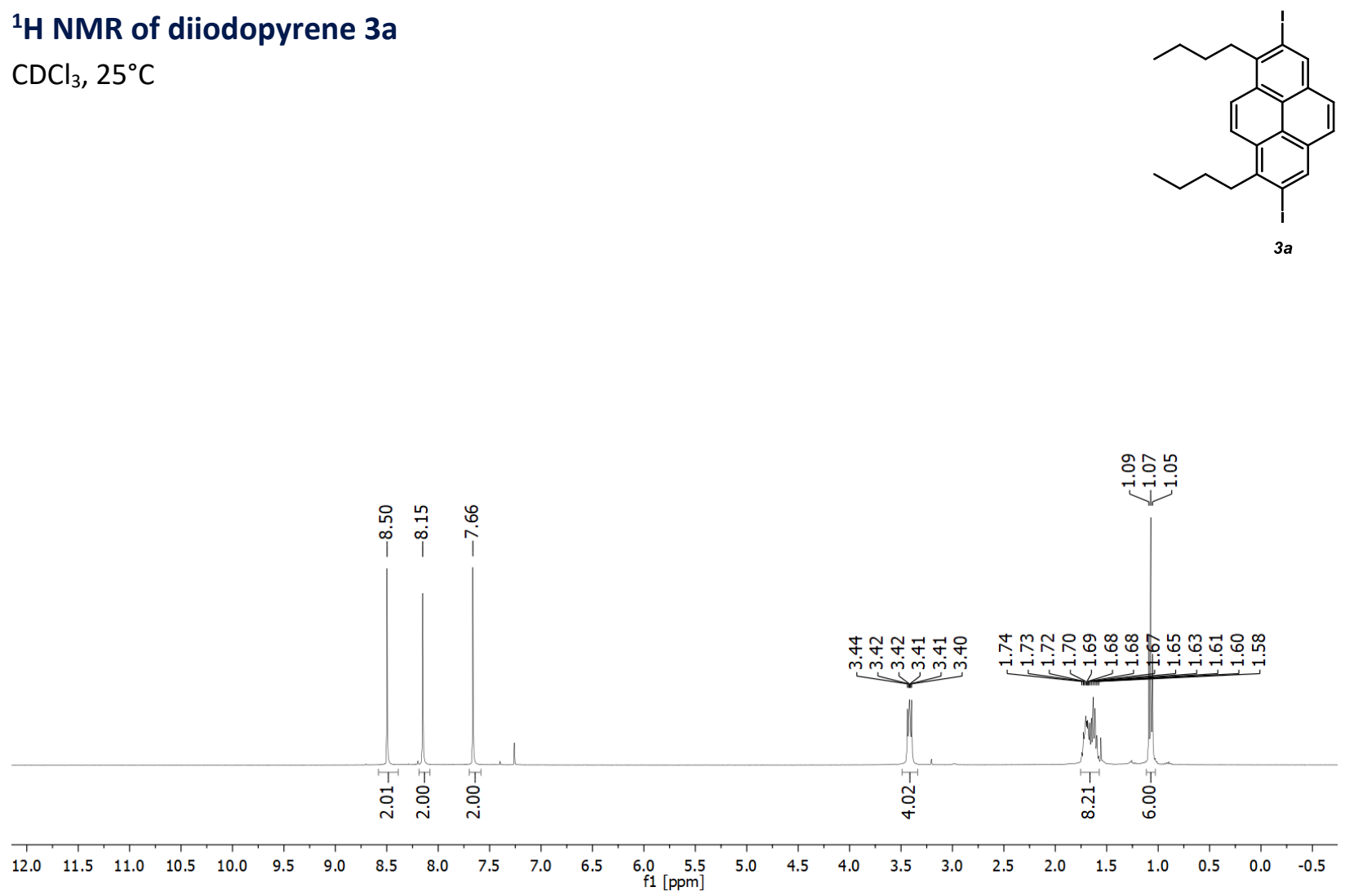

${ }^{13} \mathrm{C}$ NMR of diiodopyrene $3 \mathrm{a}$

$\mathrm{CDCl}_{3}, 25^{\circ} \mathrm{C}$

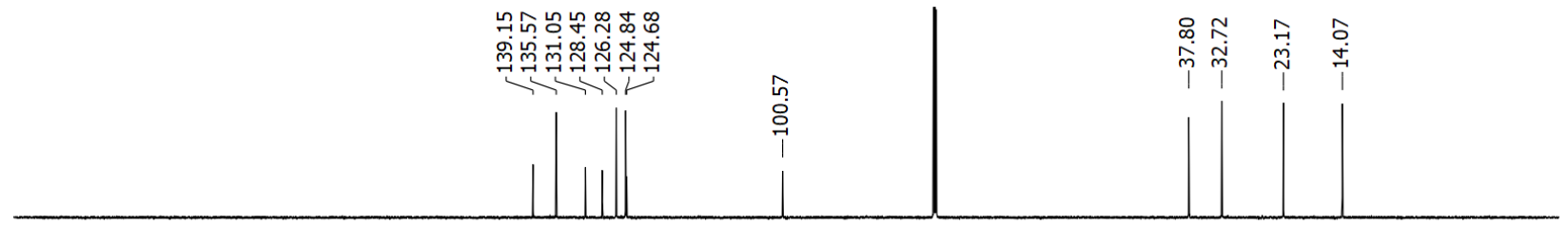

$\begin{array}{lllllllllllllllllllllll}210 & 200 & 190 & 180 & 170 & 160 & 150 & 140 & 130 & 120 & 110 & \begin{array}{l}100 \\ \mathrm{f} 1[\mathrm{ppm}]\end{array} & 90 & 80 & 70 & 60 & 50 & 40 & 30 & 20 & 10 & 0 & -10\end{array}$ 


\section{${ }^{1} \mathrm{H}$ NMR of diiodopyrene $3 \mathrm{~b}$}

$\mathrm{CDCl}_{3}, 25^{\circ} \mathrm{C}$
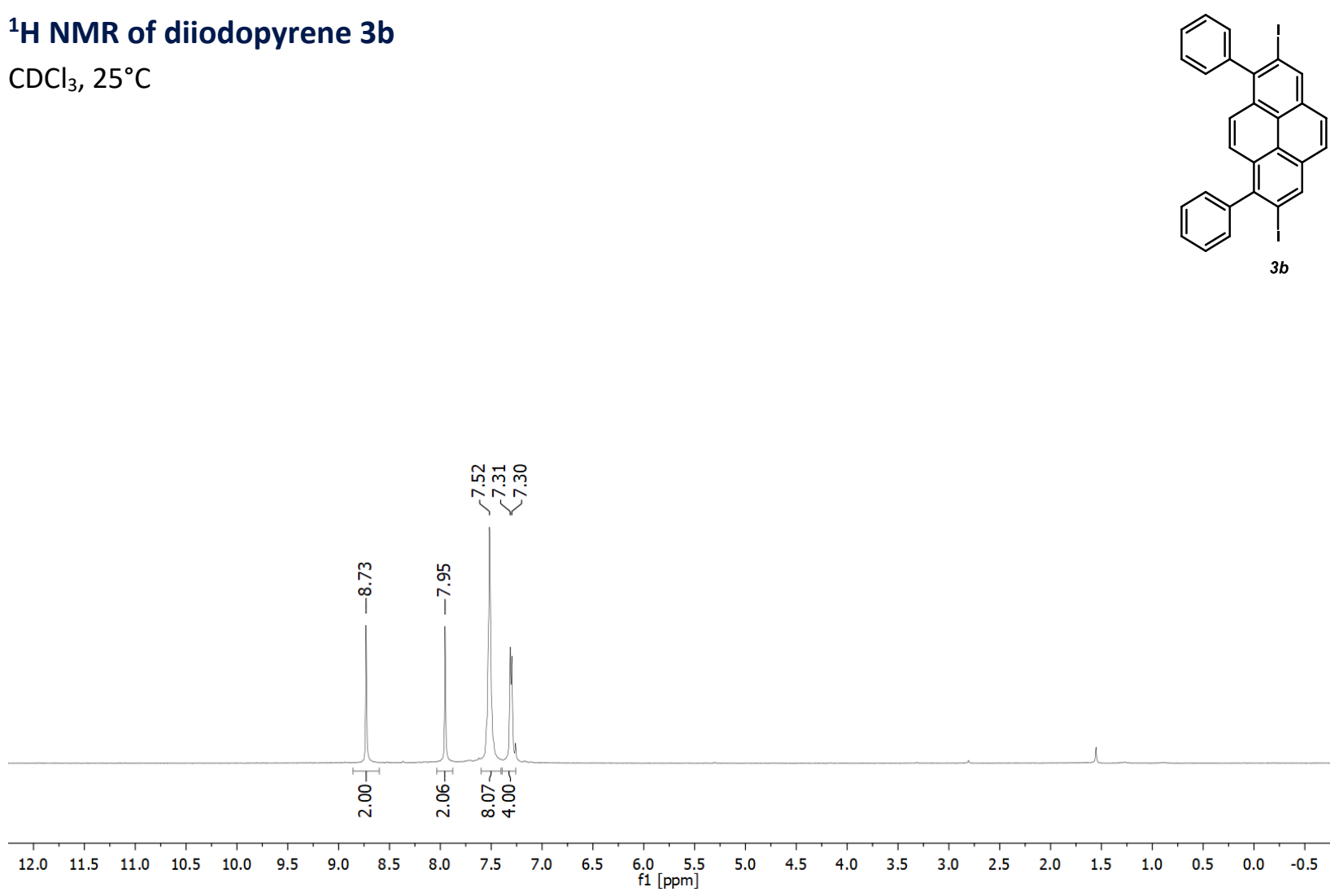

\section{${ }^{13} \mathrm{C}$ NMR of diiodopyrene $3 \mathrm{~b}$}

$\mathrm{CDCl}_{3}, 25^{\circ} \mathrm{C}$

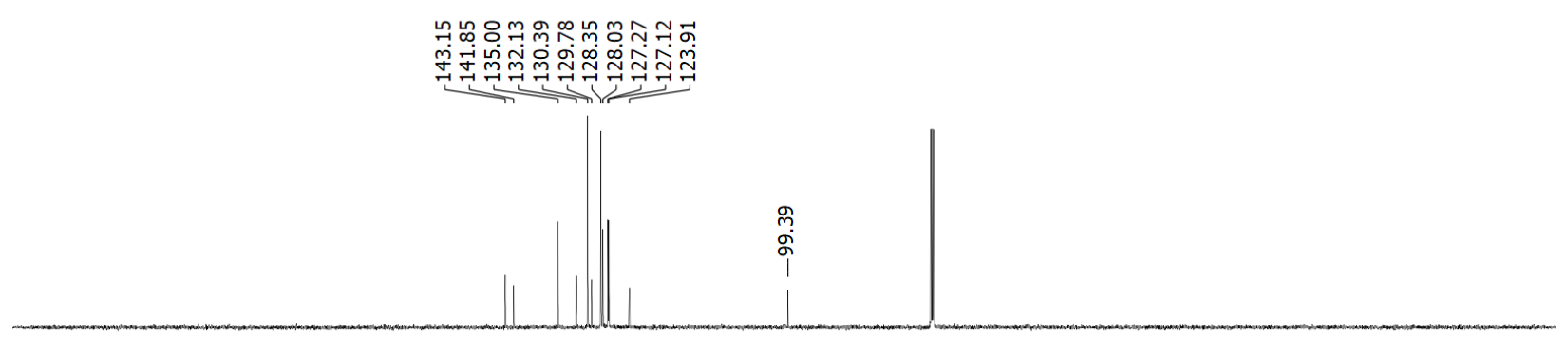

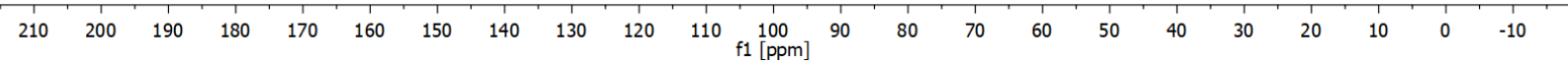




\section{${ }^{1} \mathrm{H}$ NMR of diiodopyrene $3 \mathrm{C}$}

$\mathrm{CDCl}_{3}, 25^{\circ} \mathrm{C}$

(mixture of atropisomers)
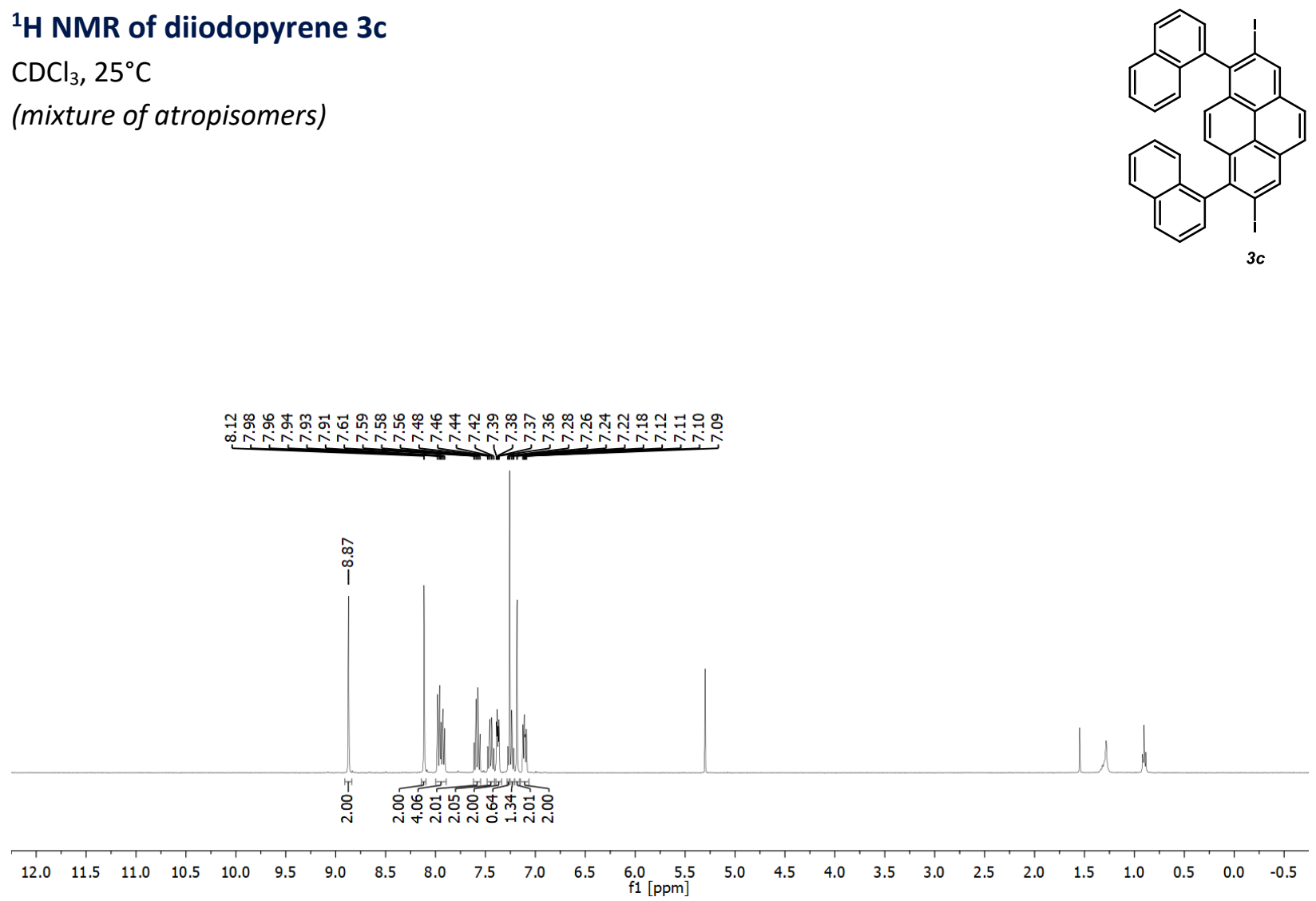

\section{${ }^{13} \mathrm{C}$ NMR of diiodopyrene $3 \mathrm{C}$}

$\mathrm{CDCl}_{3}, 25^{\circ} \mathrm{C}$
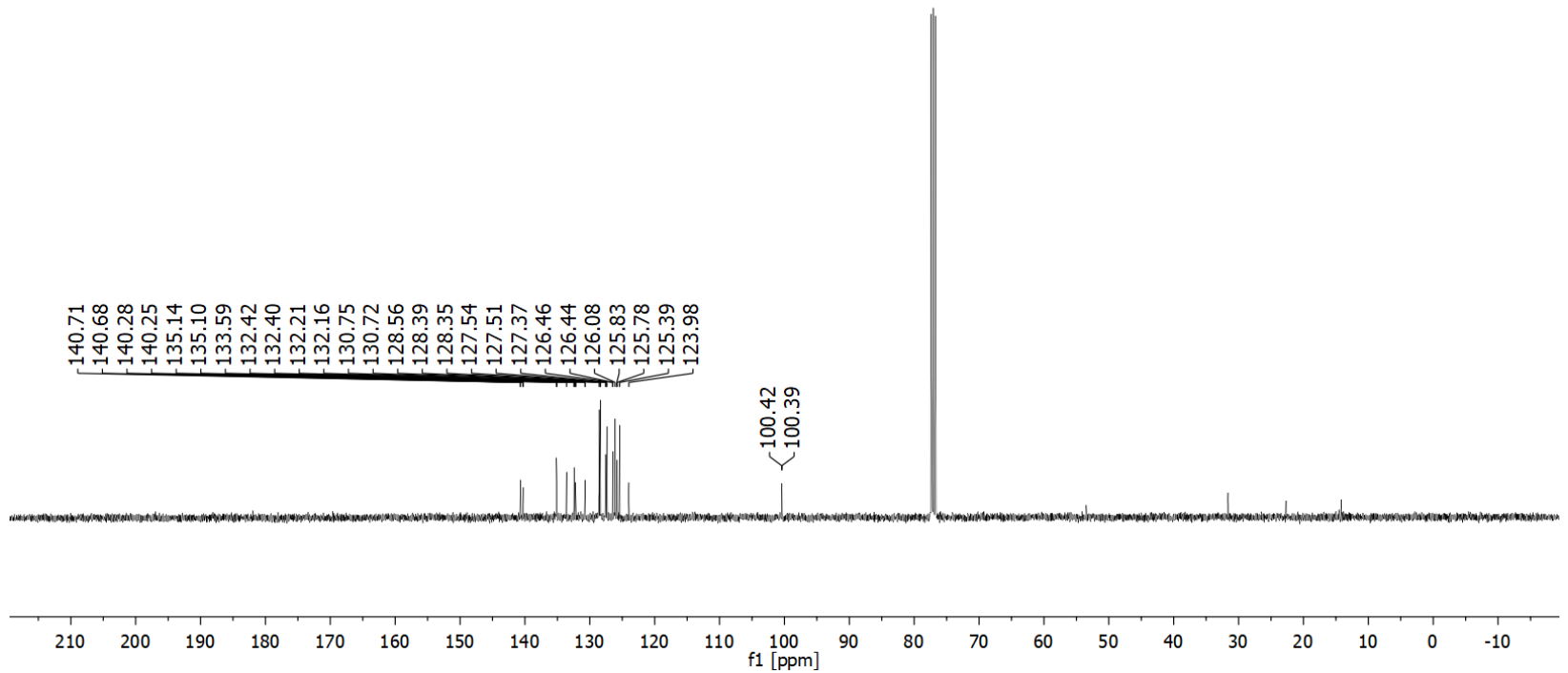


\section{${ }^{1} \mathrm{H}$ NMR of diiodopyrene $\mathbf{3 d}$}

$\mathrm{CDCl}_{3}, 25^{\circ} \mathrm{C}$
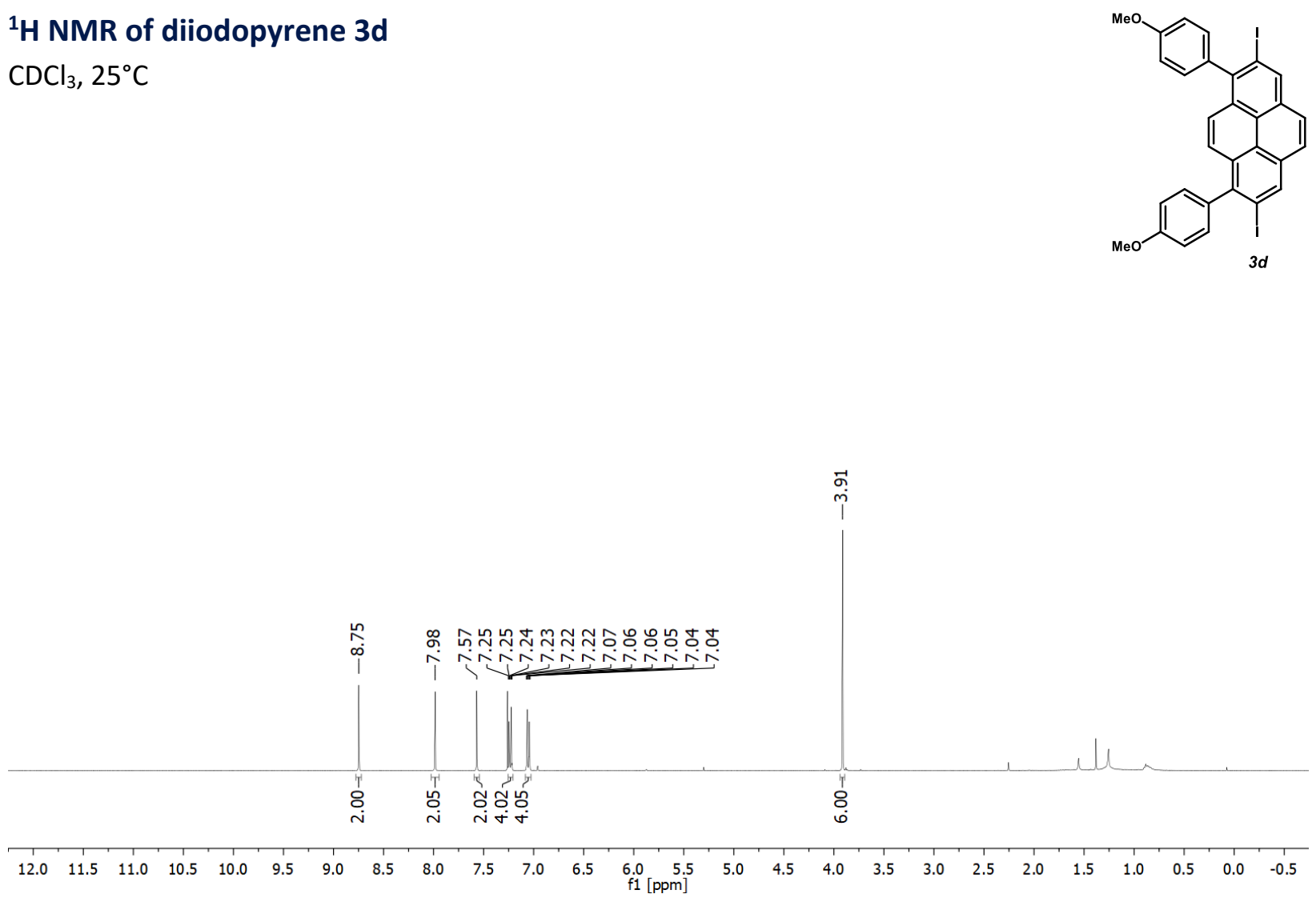

\section{${ }^{13} \mathrm{C}$ NMR of diiodopyrene $3 \mathrm{~d}$}

$\mathrm{CDCl}_{3}, 25^{\circ} \mathrm{C}$

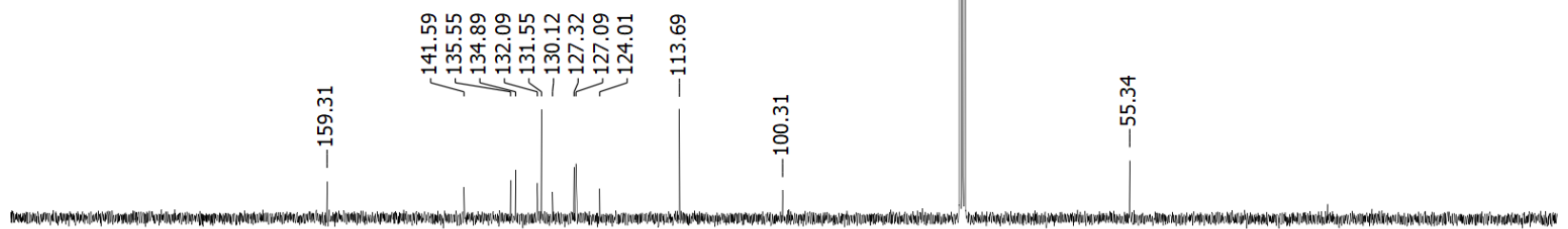

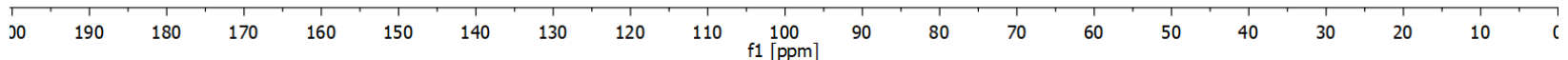




\section{${ }^{1} \mathrm{H}$ NMR of diiodopyrene $3 \mathrm{e}$}

$\mathrm{CDCl}_{3}, 25^{\circ} \mathrm{C}$
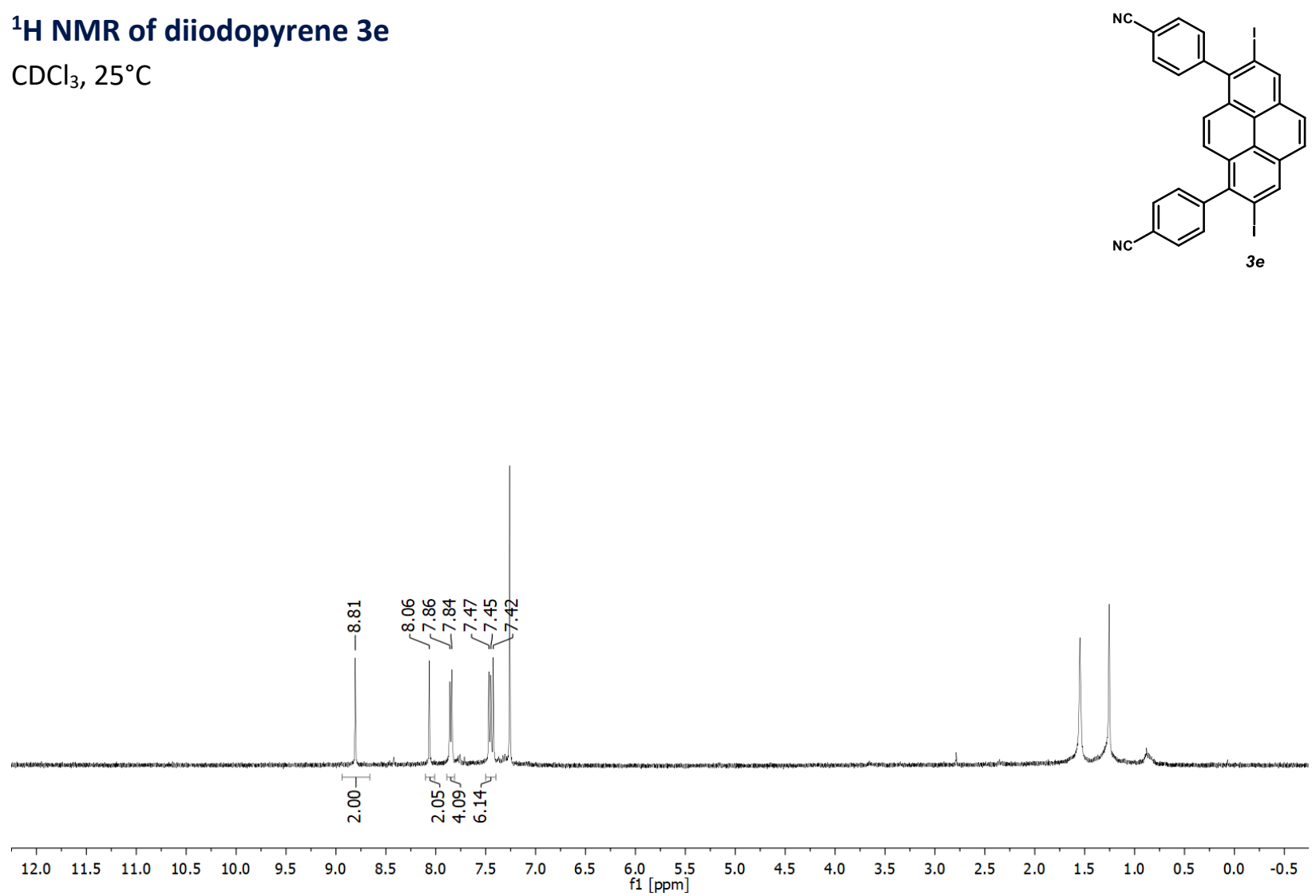

\section{${ }^{13} \mathrm{C}$ NMR of diiodopyrene $3 \mathrm{e}$}

$\mathrm{CDCl}_{3}, 25^{\circ} \mathrm{C}$
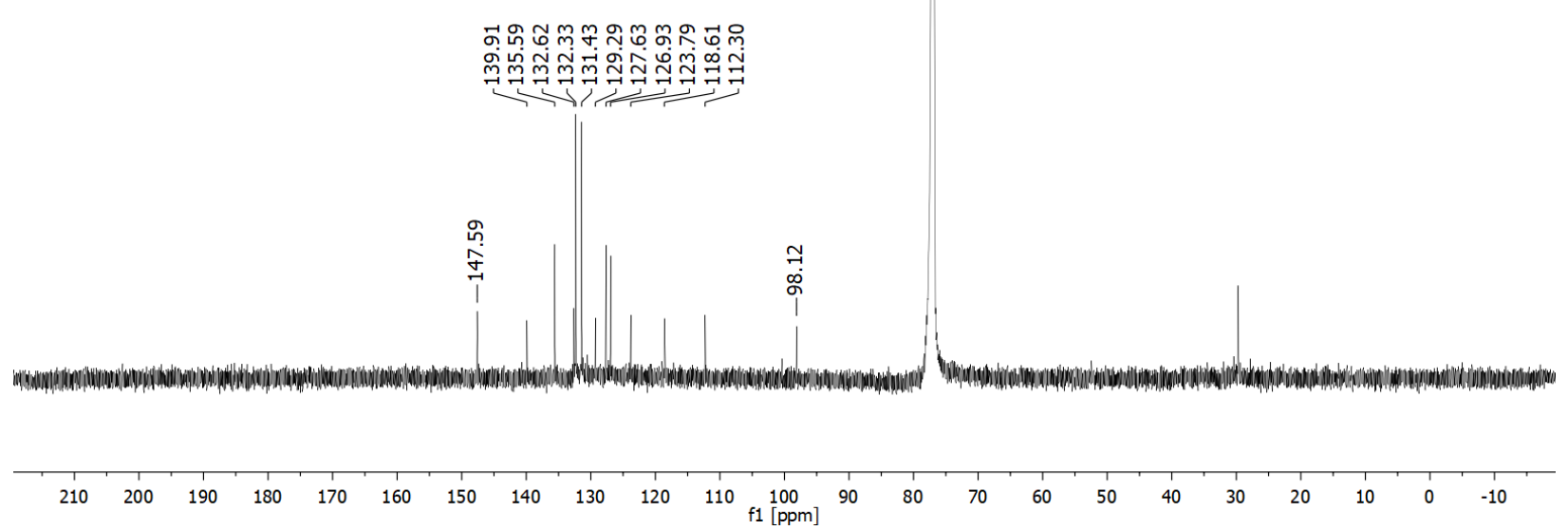


\section{${ }^{1} \mathrm{H}$ NMR of diiodobenzopyrene $\mathbf{3 g}$}

$\mathrm{CDCl}_{3}, 25^{\circ} \mathrm{C}$
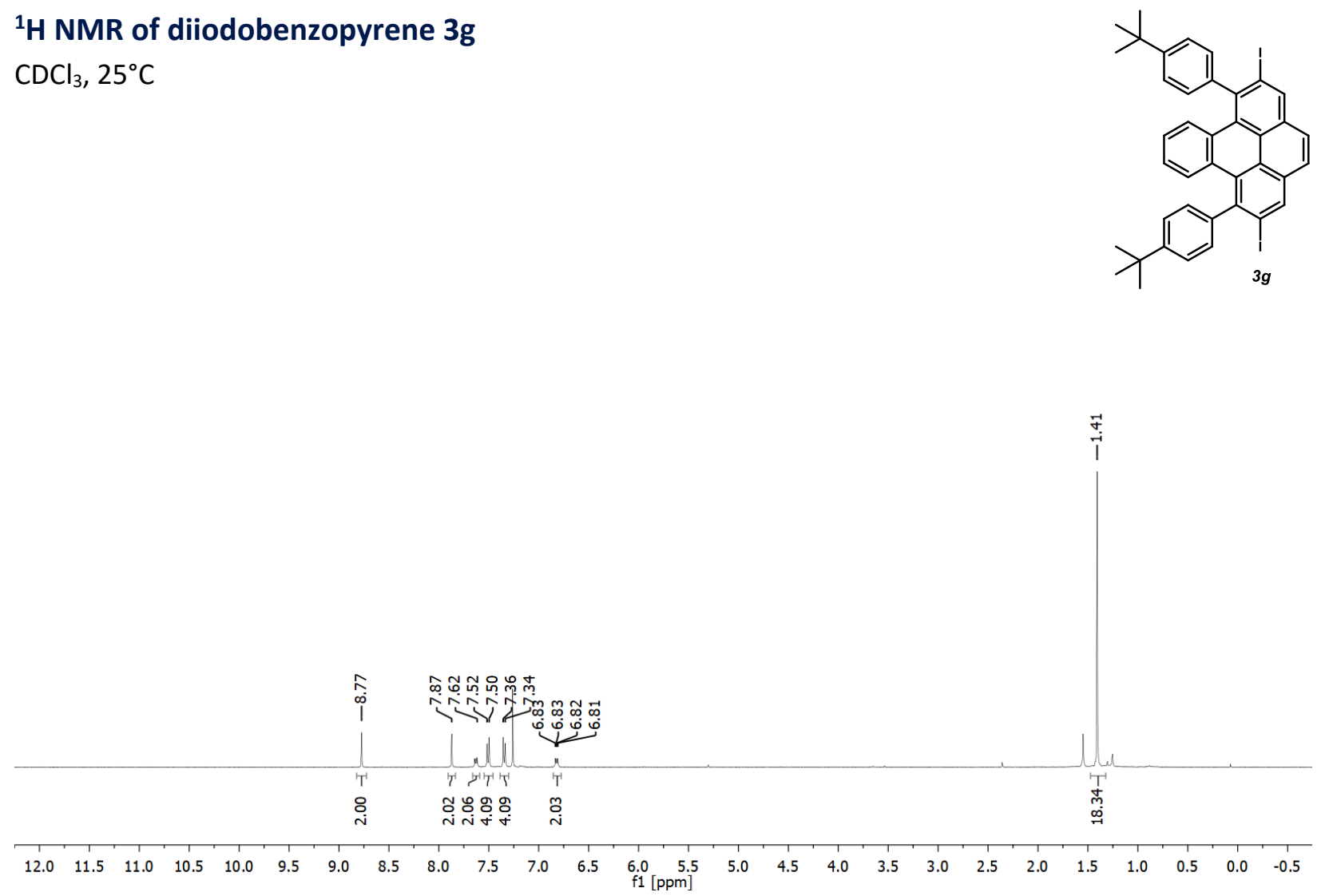

${ }^{13} \mathrm{C}$ NMR of diiodobenzopyrene $3 \mathrm{~g}$

$\mathrm{CDCl}_{3}, 25^{\circ} \mathrm{C}$

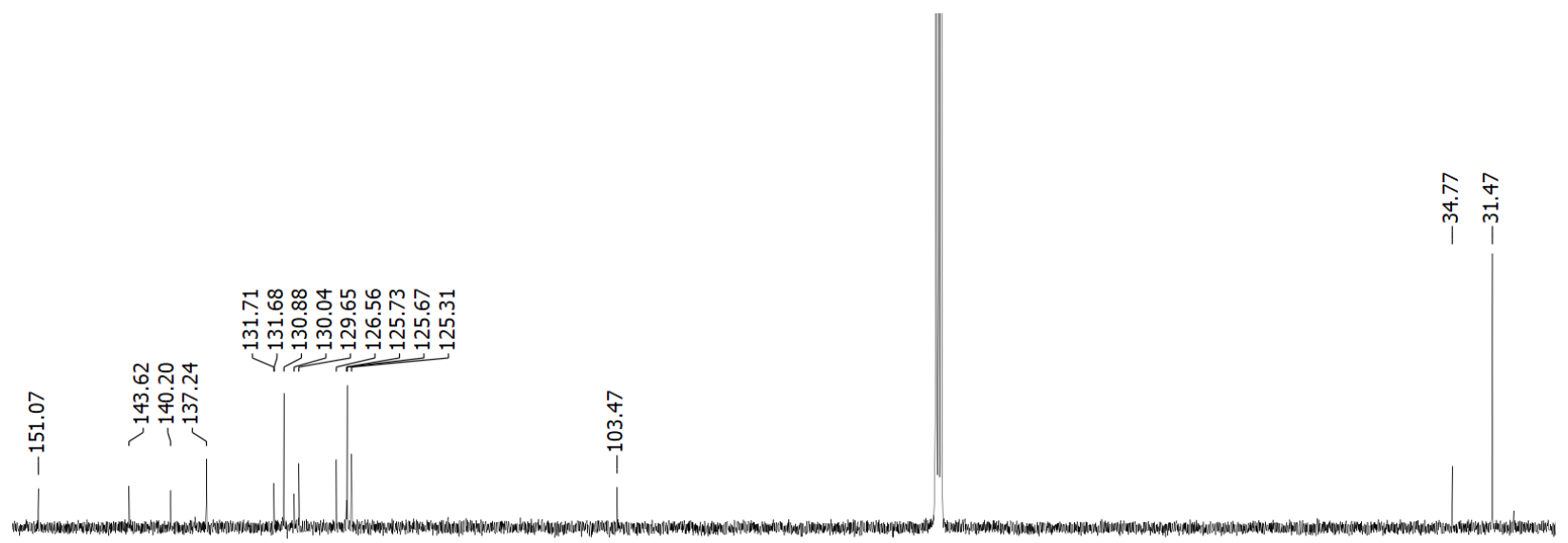

$\begin{array}{llllllllllllllllllllllllll}150 & 145 & 140 & 135 & 130 & 125 & 120 & 115 & 110 & 105 & 100 & 95 & 90 & 85 & 80 & 75 & 70 & 65 & 60 & 55 & 50 & 45 & 40 & 35 & 30 & \end{array}$ 


\section{${ }^{1} \mathbf{H}$ NMR of diiodoperopyrene $3 \mathbf{i}$}

$\mathrm{CDCl}_{3}, 25^{\circ} \mathrm{C}$
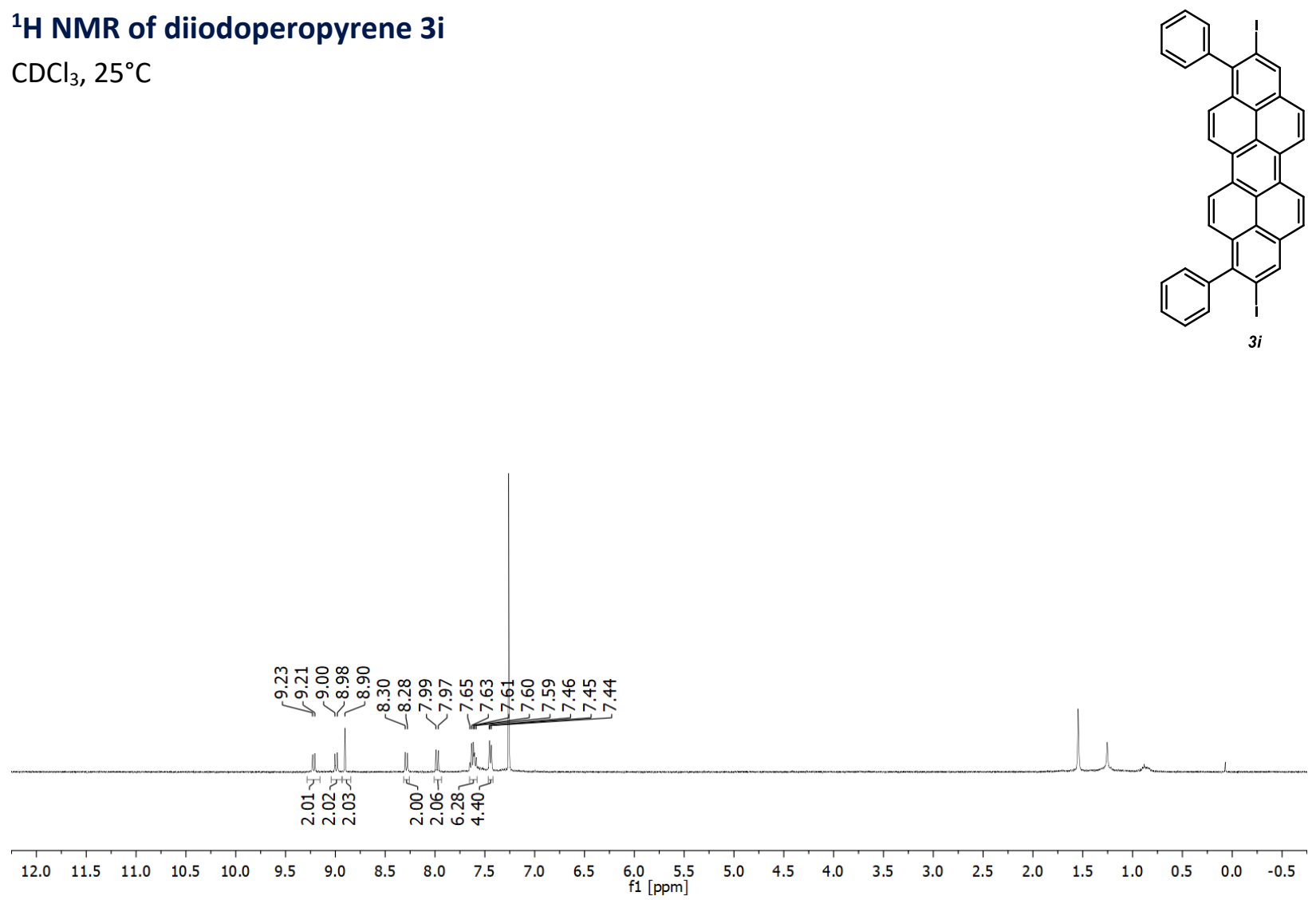

${ }^{13} \mathrm{C}$ NMR of diiodoperopyrene $3 \mathbf{i}$

$\mathrm{CDCl}_{3}, 25^{\circ} \mathrm{C}$

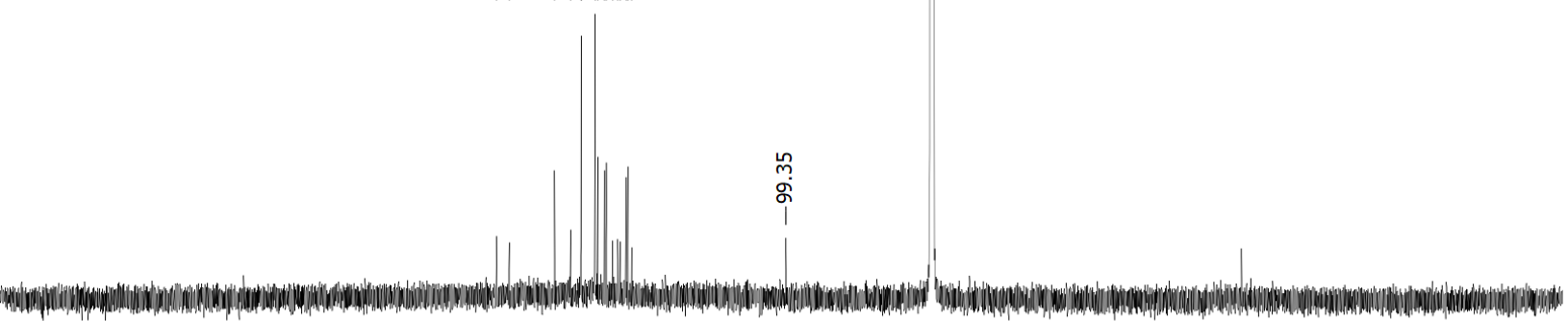

$\begin{array}{lllllllllllllllllllllll}210 & 200 & 190 & 180 & 170 & 160 & 150 & 140 & 130 & 120 & 110 & 100 & 90 & 80 & 70 & 60 & 50 & 40 & 30 & 20 & 10 & 0 & -10\end{array}$


${ }^{1} \mathbf{H}$ NMR of diiodopyrene $3 \mathbf{j}$

$\mathrm{CDCl}_{3}, 25^{\circ} \mathrm{C}$
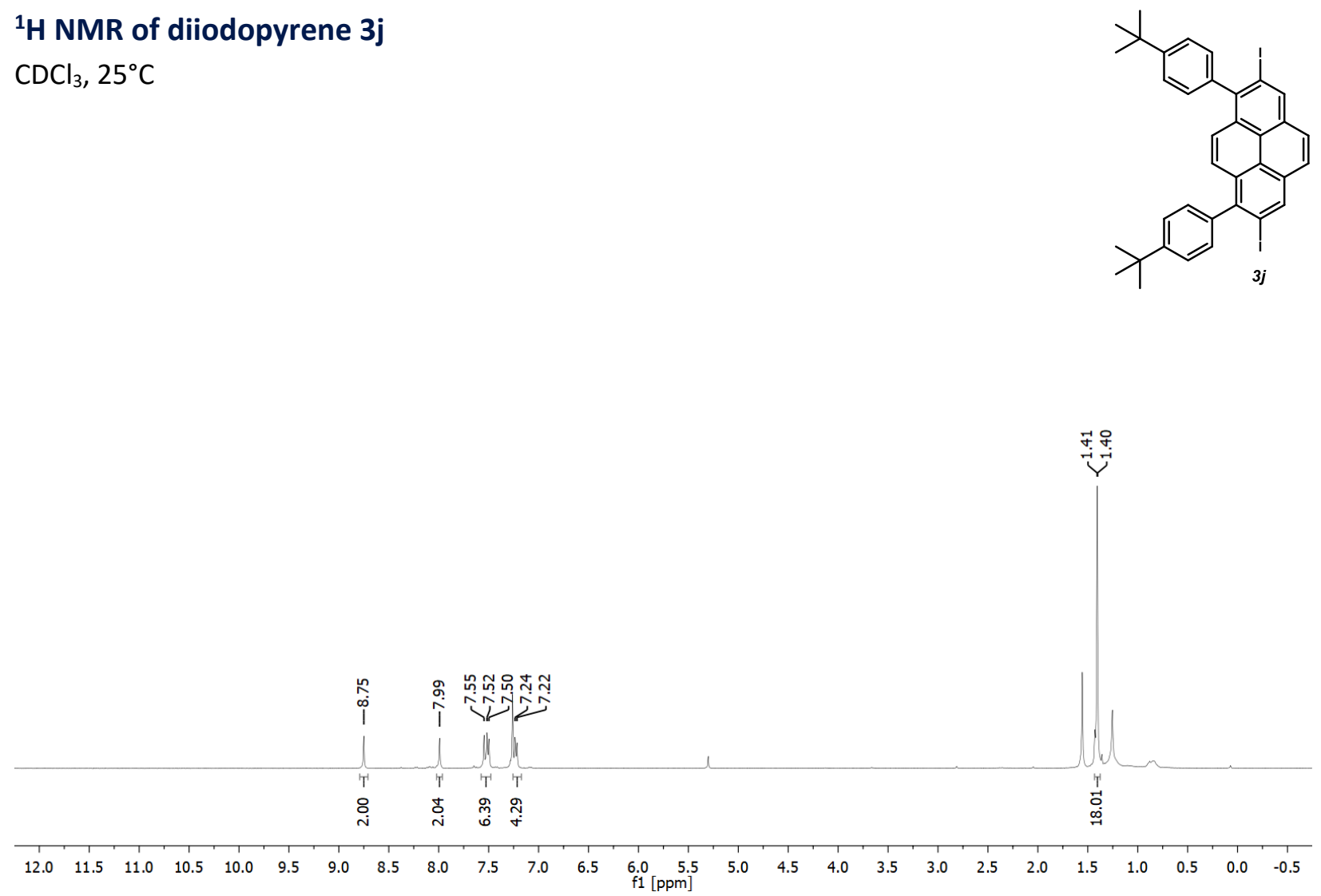

\section{${ }^{13} \mathrm{C}$ NMR of diiodopyrene $3 \mathbf{j}$}

$\mathrm{CDCl}_{3}, 25^{\circ} \mathrm{C}$

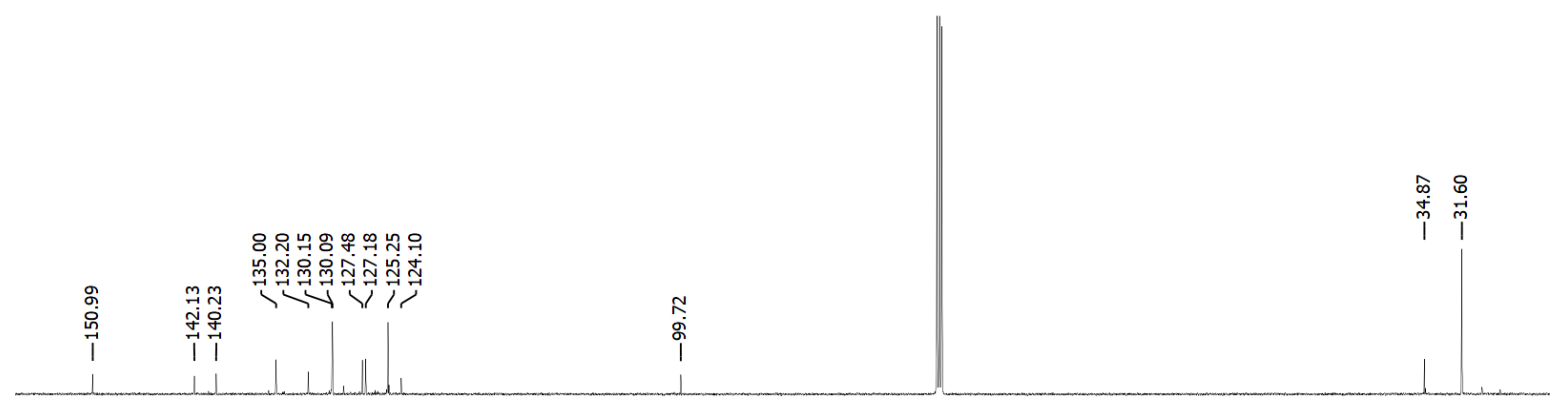

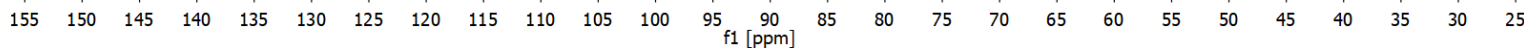


${ }^{1} \mathrm{H}$ NMR of pyrene 4a

$\mathrm{CDCl}_{3}, 25^{\circ} \mathrm{C}$

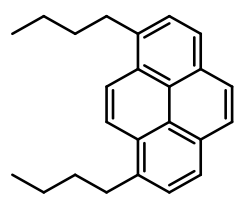

$4 a$

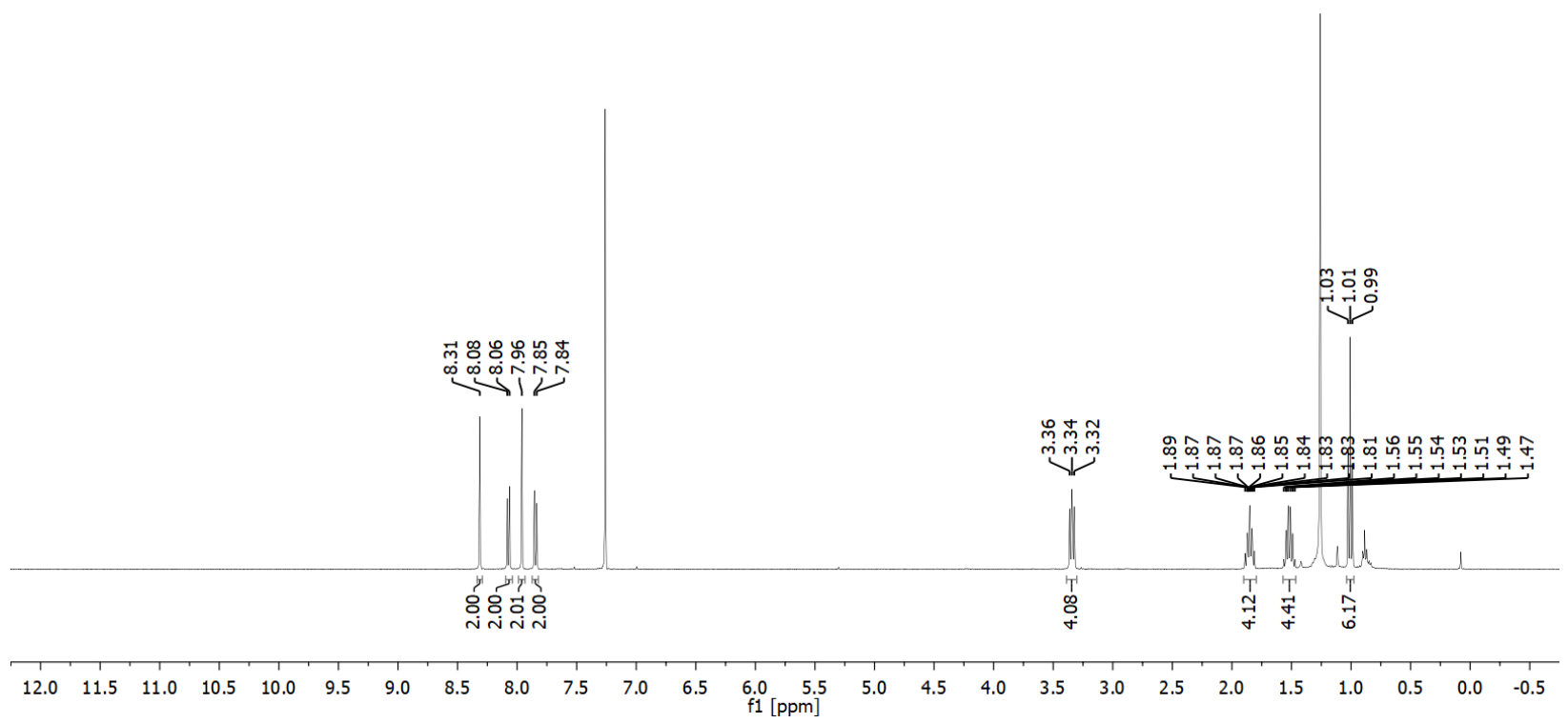

${ }^{13} \mathrm{C}$ NMR of distannylpyrene $2 \mathrm{a}$

$\mathrm{CDCl}_{3}, 25^{\circ} \mathrm{C}$

कำำำ

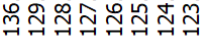

구국

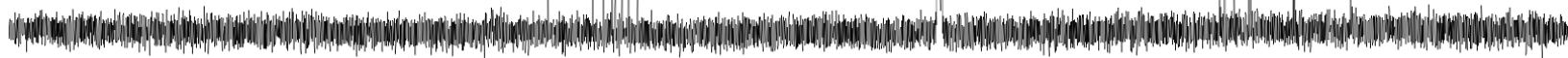

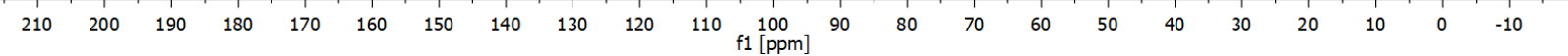


${ }^{1} \mathrm{H}$ NMR of pyrene $4 \mathrm{~b}$

$\mathrm{CDCl}_{3}, 25^{\circ} \mathrm{C}$
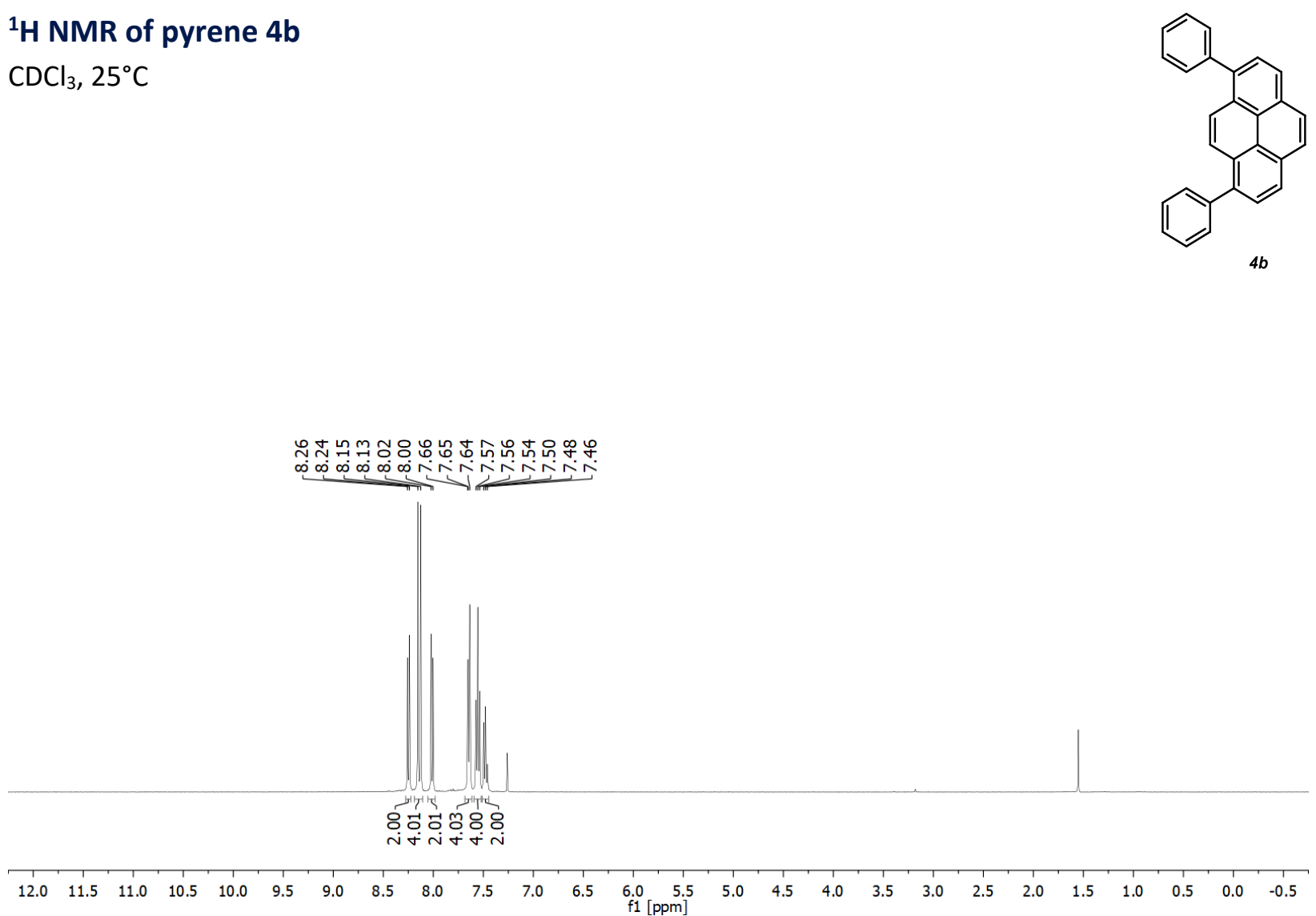

${ }^{13} \mathrm{C}$ NMR of pyrene $4 \mathrm{~b}$

$\mathrm{CDCl}_{3}, 25^{\circ} \mathrm{C}$

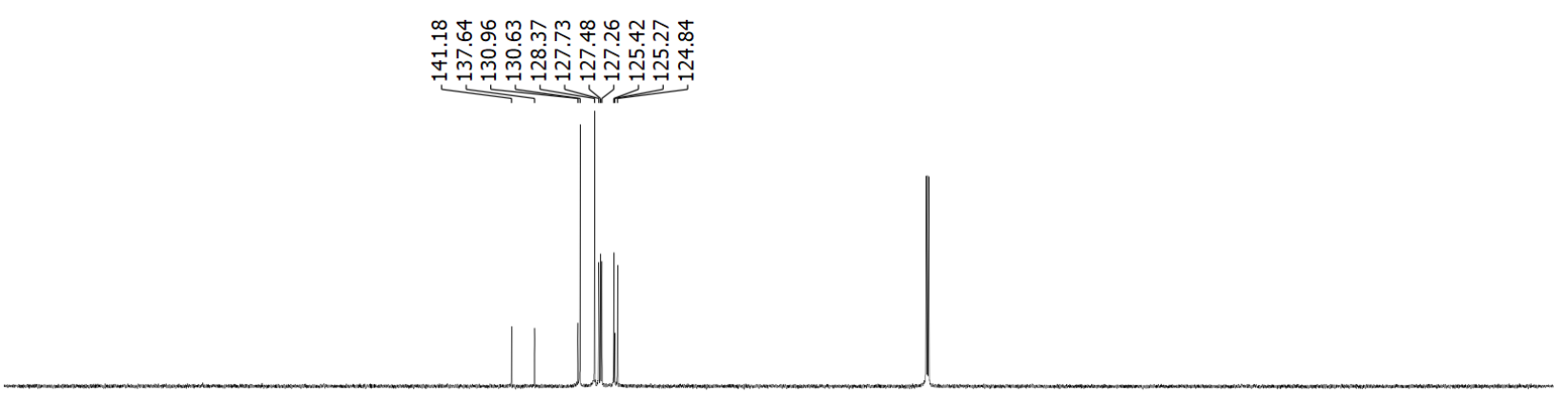

$\begin{array}{llllllllllllllllllllllllllll}210 & 200 & 190 & 180 & 170 & 160 & 150 & 140 & 130 & 120 & 110 & 100 & 90 & 80 & 70 & 60 & 50 & 40 & 30 & 20 & 10 & 0 & -10 & \end{array}$ 


\section{${ }^{1} \mathrm{H}$ NMR of pyrene $4 \mathrm{C}$}

$\mathrm{CDCl}_{3}, 25^{\circ} \mathrm{C}$

(mixture of atropisomers)
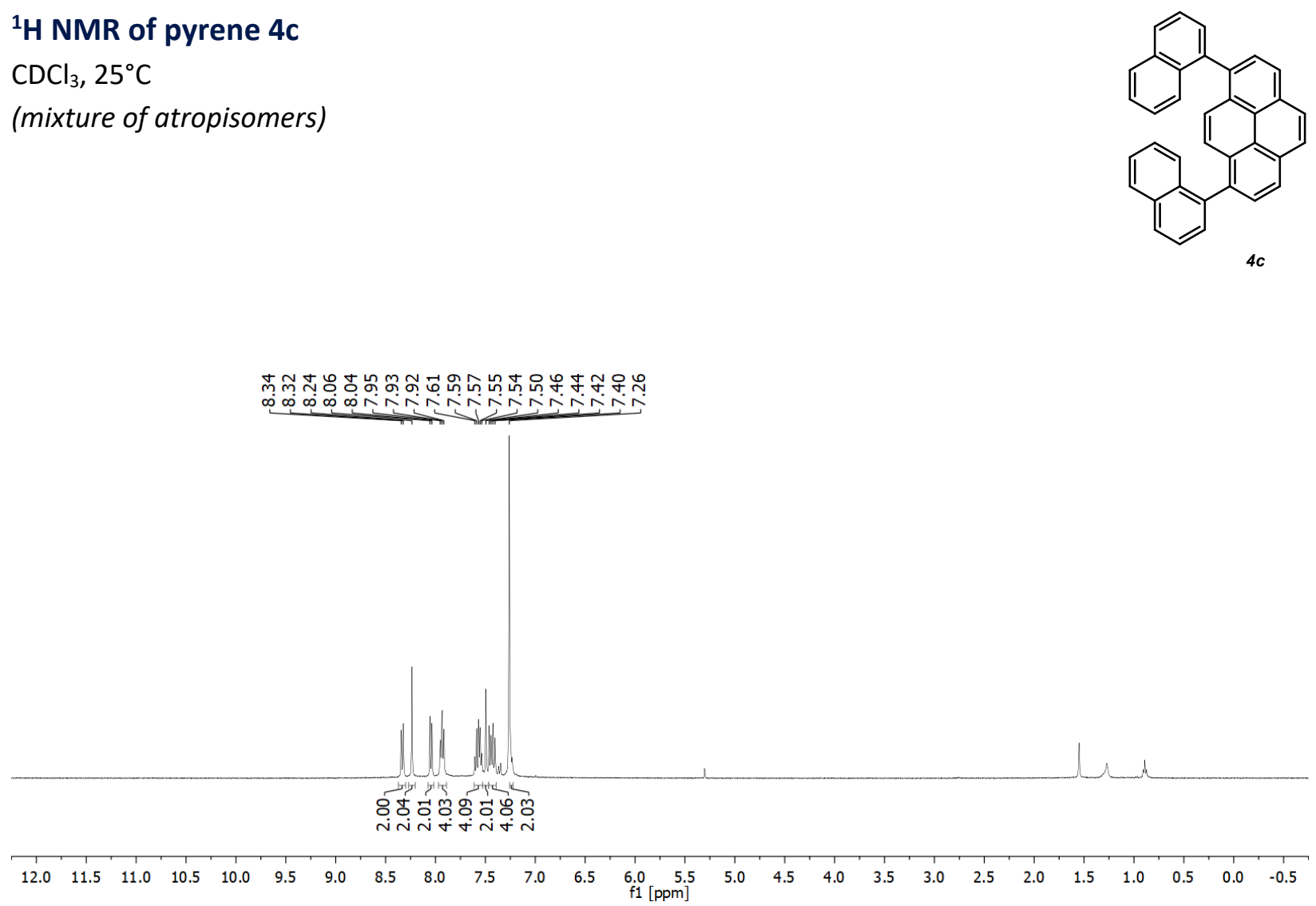

${ }^{13} \mathrm{C}$ NMR of pyrene 4c

$\mathrm{CDCl}_{3}, 25^{\circ} \mathrm{C}$
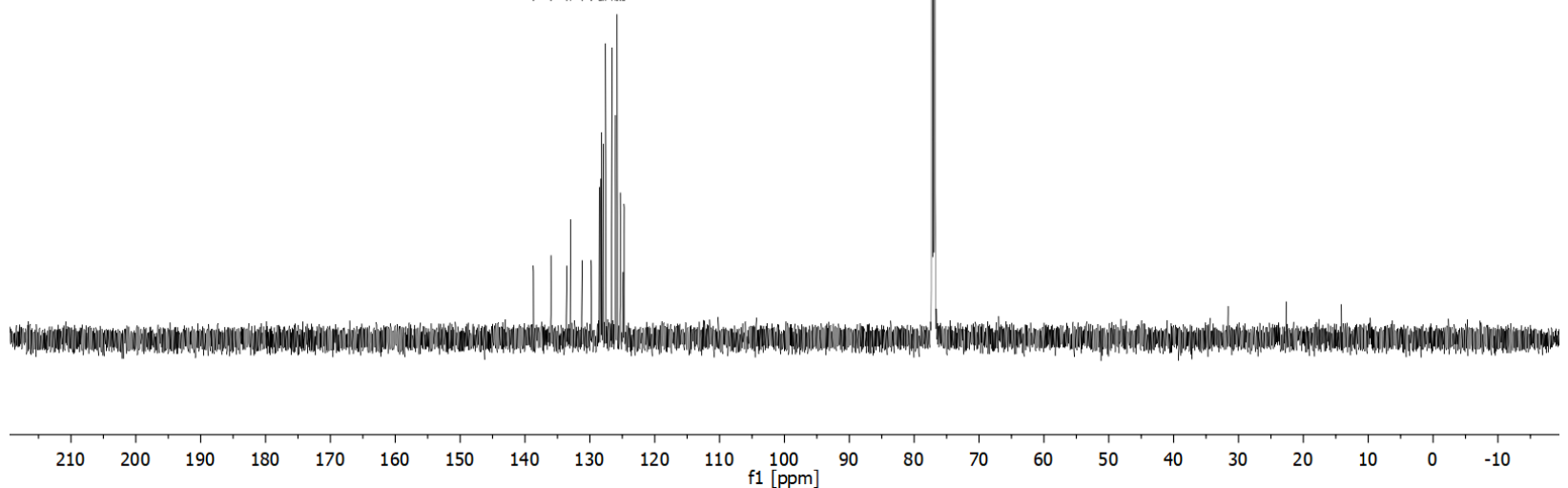


\section{${ }^{1} \mathrm{H}$ NMR of pyrene $4 d$}

$\mathrm{CDCl}_{3}, 25^{\circ} \mathrm{C}$
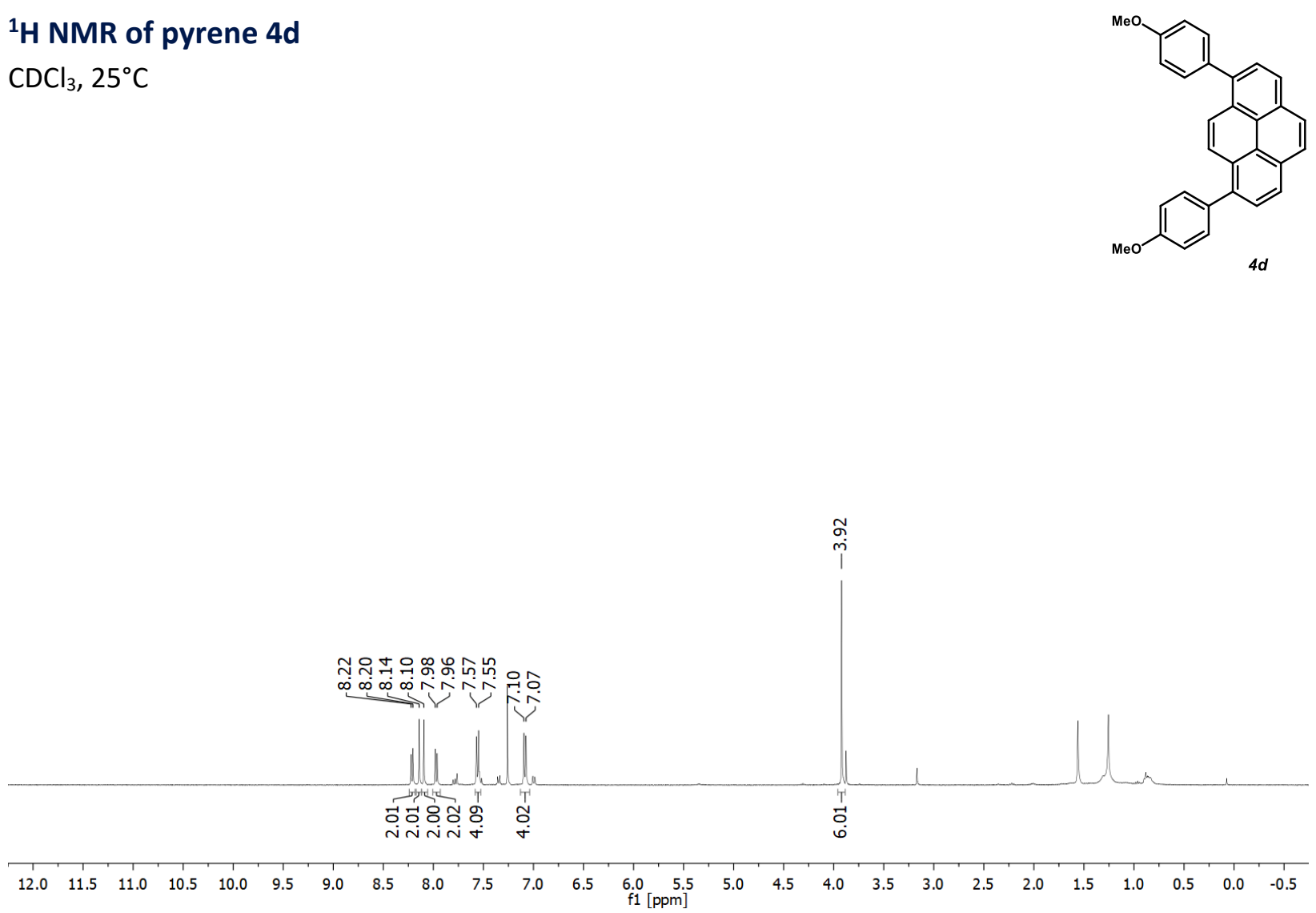

\section{${ }^{13} \mathrm{C}$ NMR of pyrene $4 \mathrm{~d}$}

$\mathrm{CDCl}_{3}, 25^{\circ} \mathrm{C}$

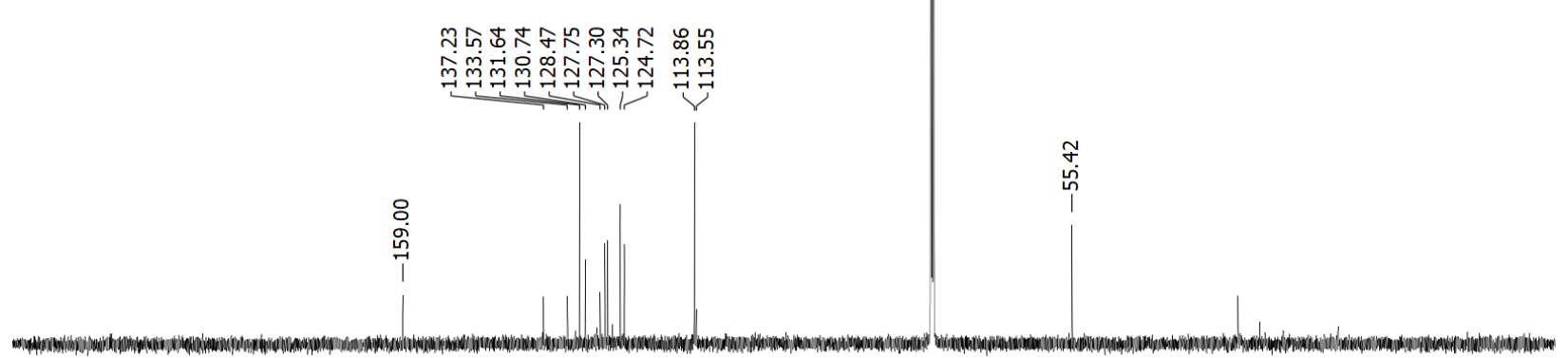




\section{${ }^{1} \mathrm{H}$ NMR of pyrene $4 \mathrm{e}$}

$\mathrm{CDCl}_{3}, 25^{\circ} \mathrm{C}$
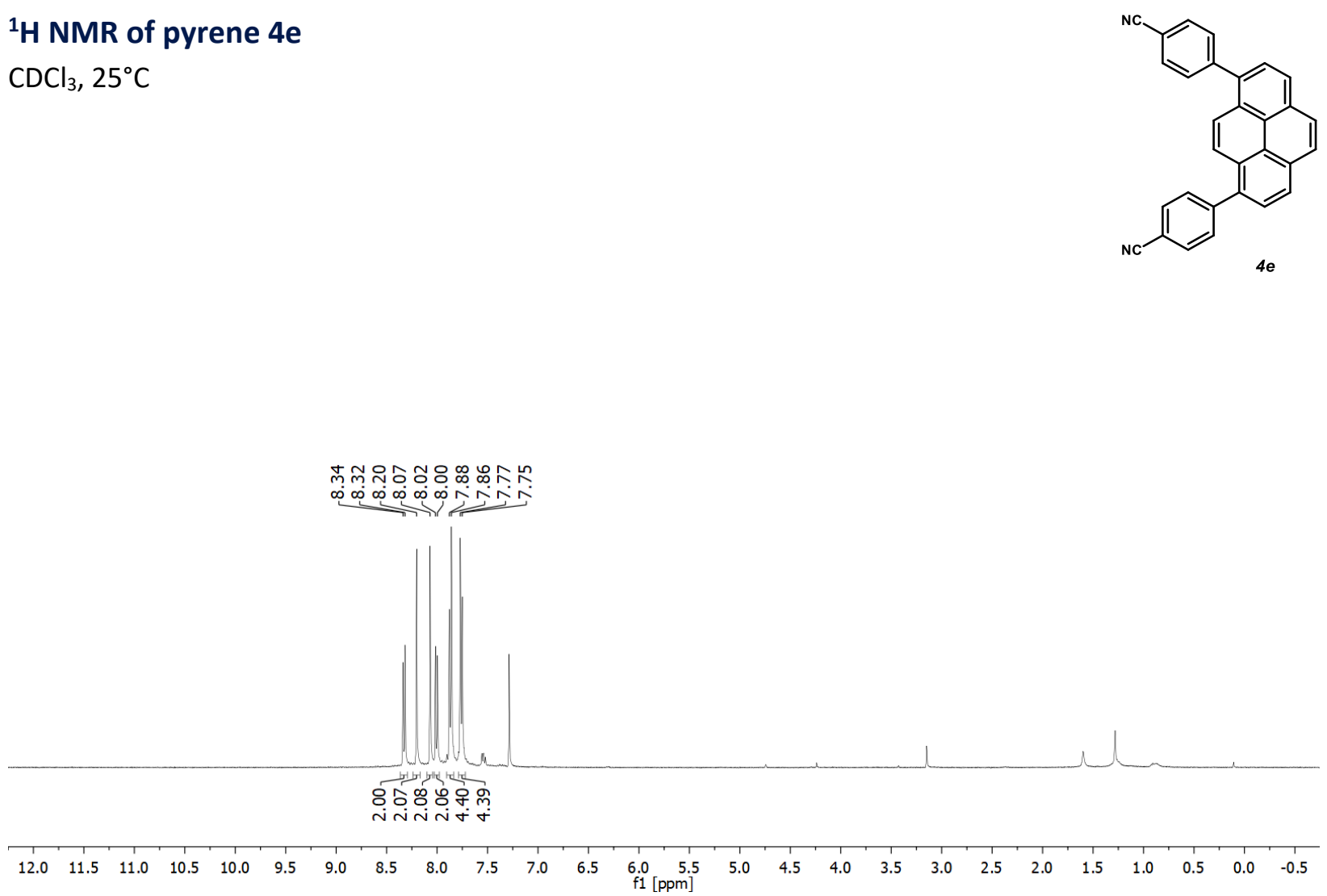

\section{${ }^{13} \mathrm{C}$ NMR of pyrene $4 \mathrm{e}$}

$\mathrm{CDCl}_{3}, 25^{\circ} \mathrm{C}$

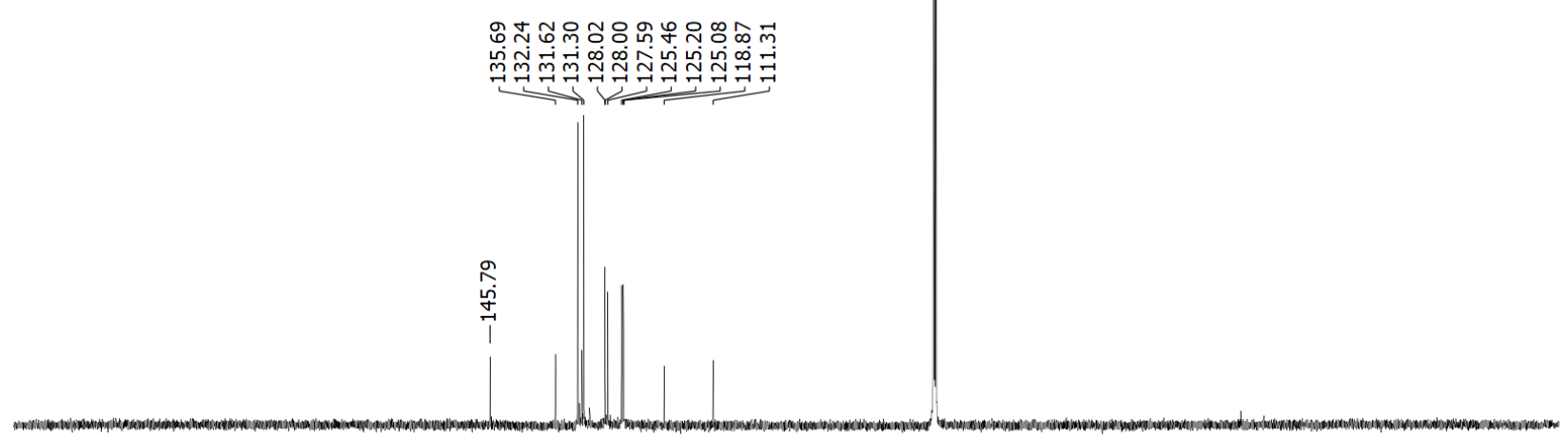

$\begin{array}{llllllll}210 & 200 & 190 & 180 & 170 & 160 & 150 & 140\end{array}$

$10 \stackrel{100}{100} 90$

80

60

$50 \quad 40 \quad 30$

$\begin{array}{llll}1 & 1 & 1 & 1\end{array}$ 


\section{${ }^{1} \mathrm{H}$ NMR of benzopyrene $4 \mathrm{~g}$}

$\mathrm{CDCl}_{3}, 25^{\circ} \mathrm{C}$
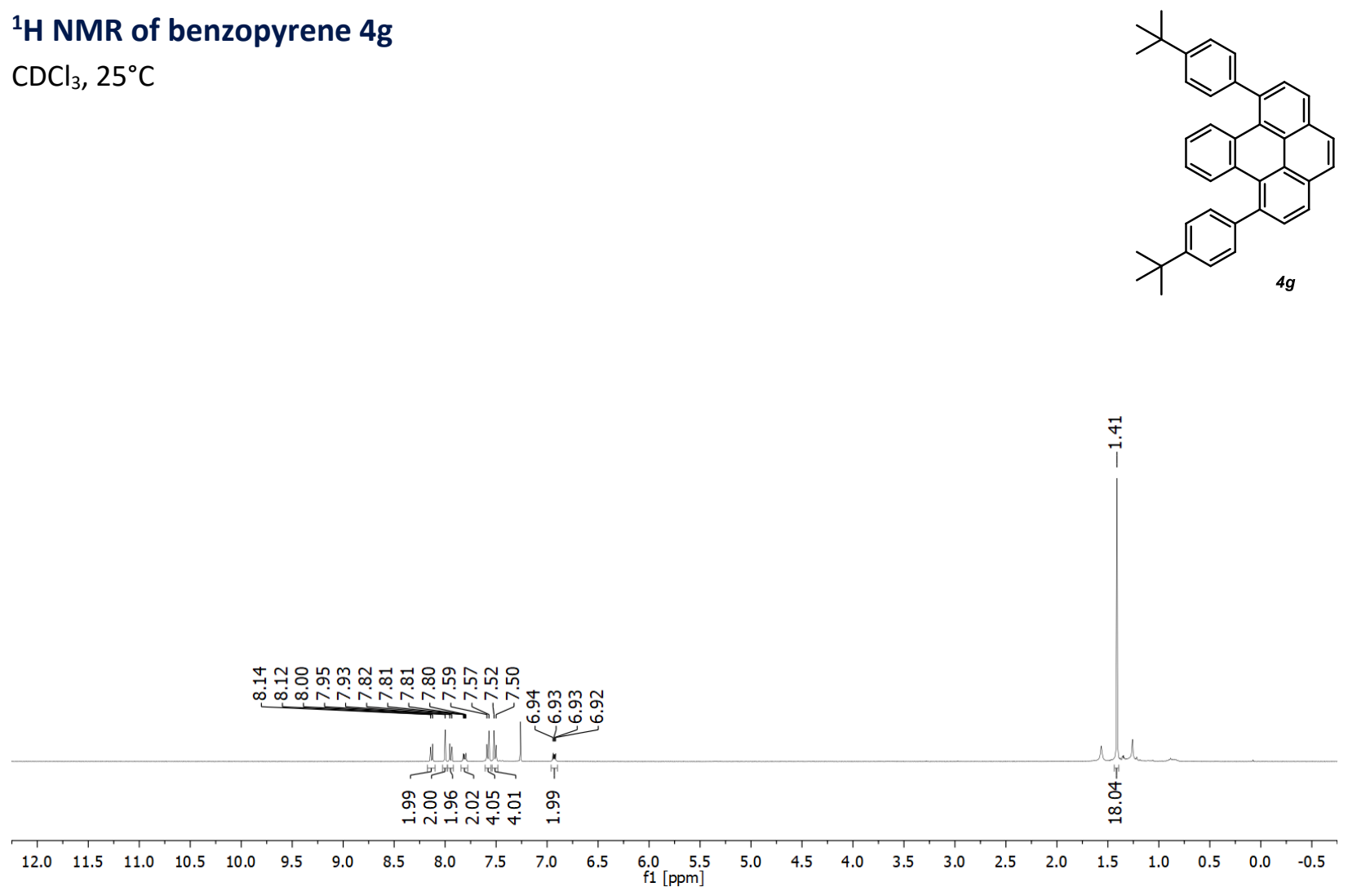

${ }^{13} \mathrm{C}$ NMR of benzopyrene $2 \mathrm{~g}$

$\mathrm{CDCl}_{3}, 25^{\circ} \mathrm{C}$

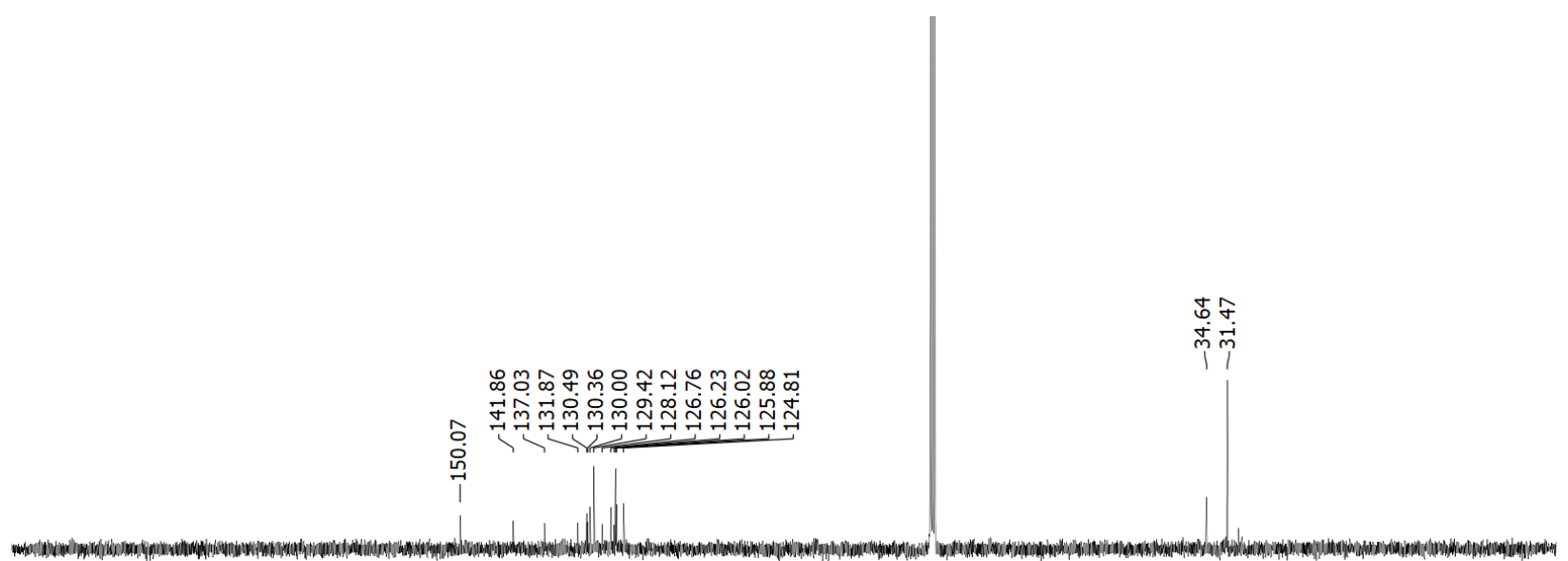

$\begin{array}{llllllllllll}210 & 200 & 190 & 180 & 170 & 160 & 150 & 140 & 130 & 120 & 110 & \begin{array}{l}100 \\ \mathrm{f} 1[\mathrm{ppm}]\end{array}\end{array}$

80

60




\section{${ }^{1} \mathrm{H}$ NMR of perylene $4 \mathrm{~h}$}

$\mathrm{CDCl}_{3}, 25^{\circ} \mathrm{C}$
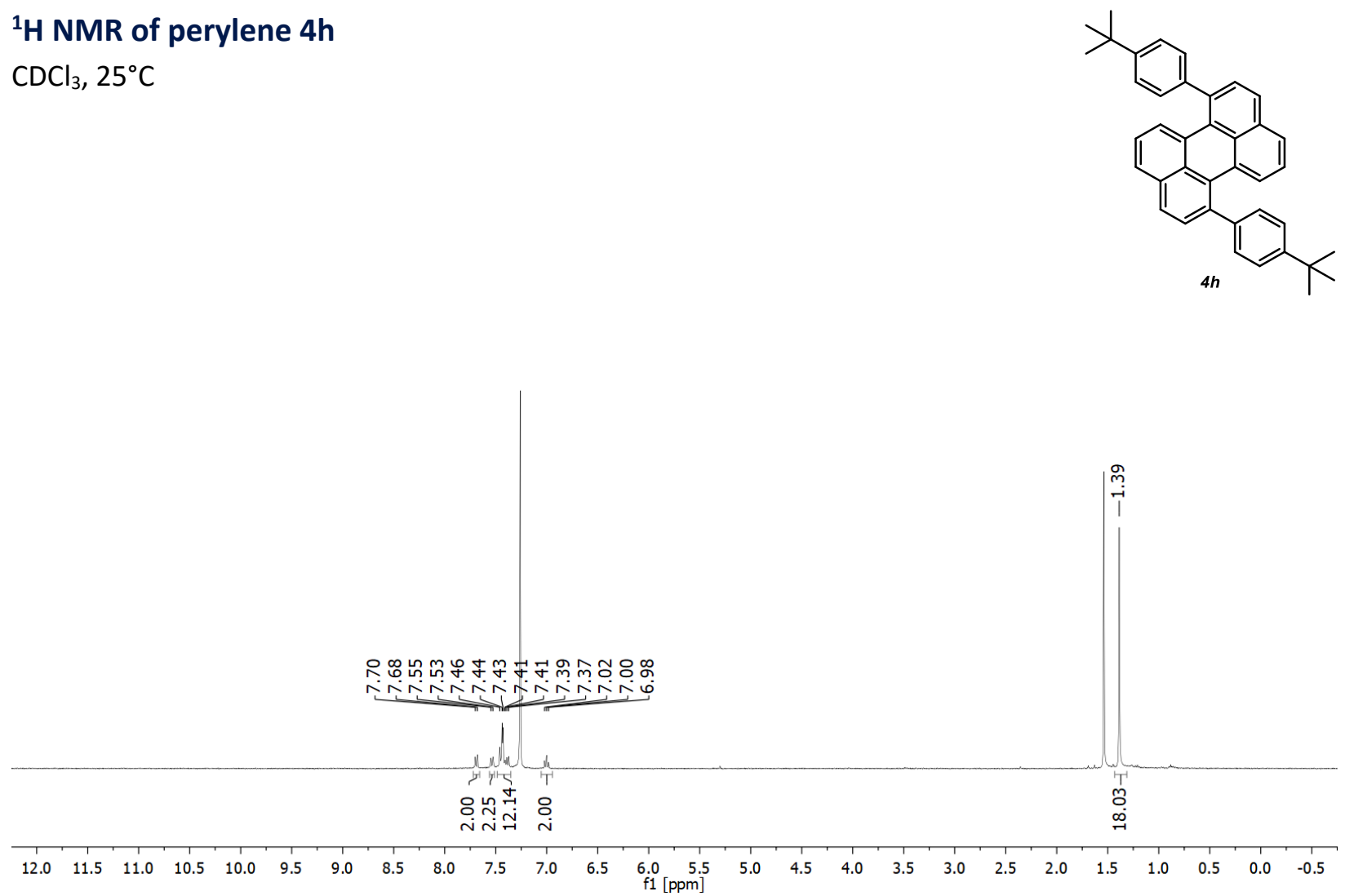

\section{${ }^{13} \mathrm{C}$ NMR of perylene $4 \mathrm{~h}$}

$\mathrm{CDCl}_{3}, 25^{\circ} \mathrm{C}$

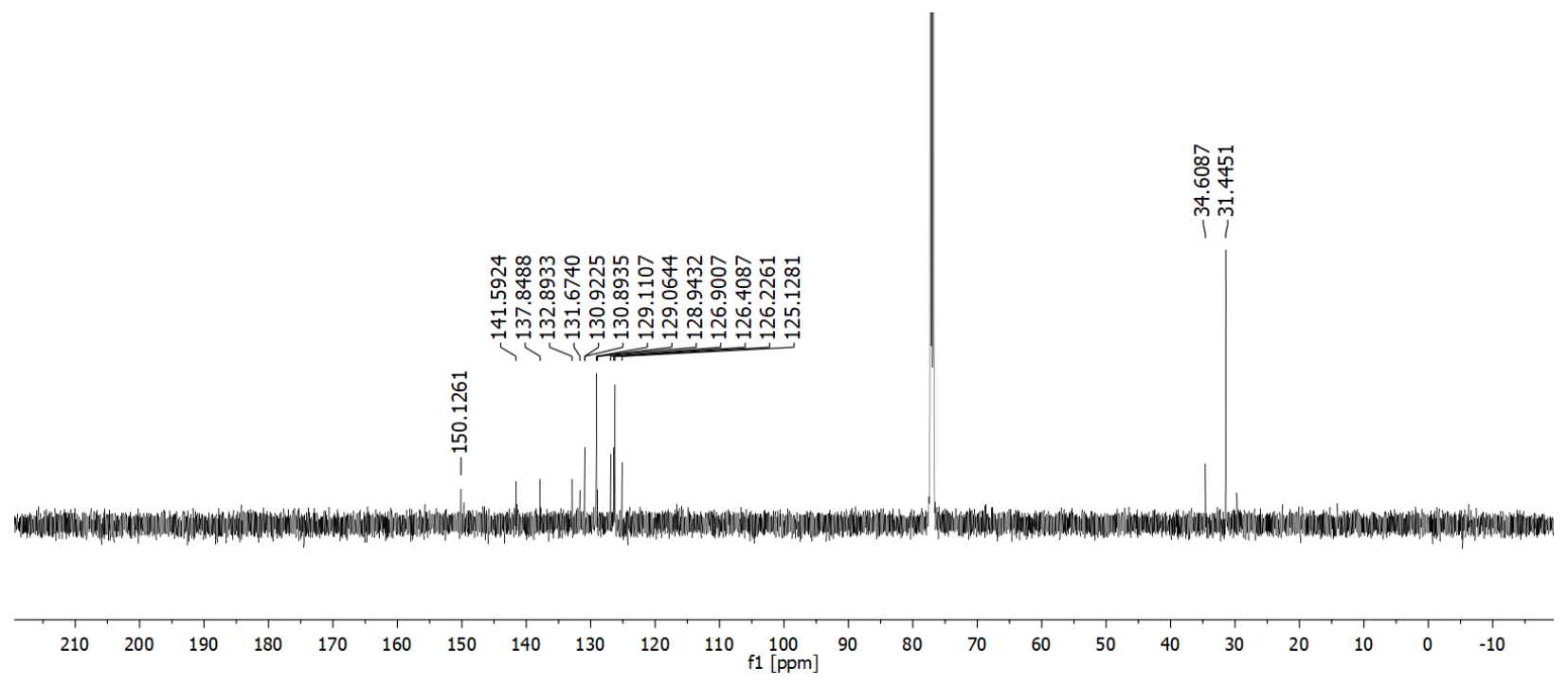




\section{${ }^{1} \mathrm{H}$ NMR of peropyrene $4 \mathbf{i}$}

$\mathrm{CDCl}_{3}, 25^{\circ} \mathrm{C}$
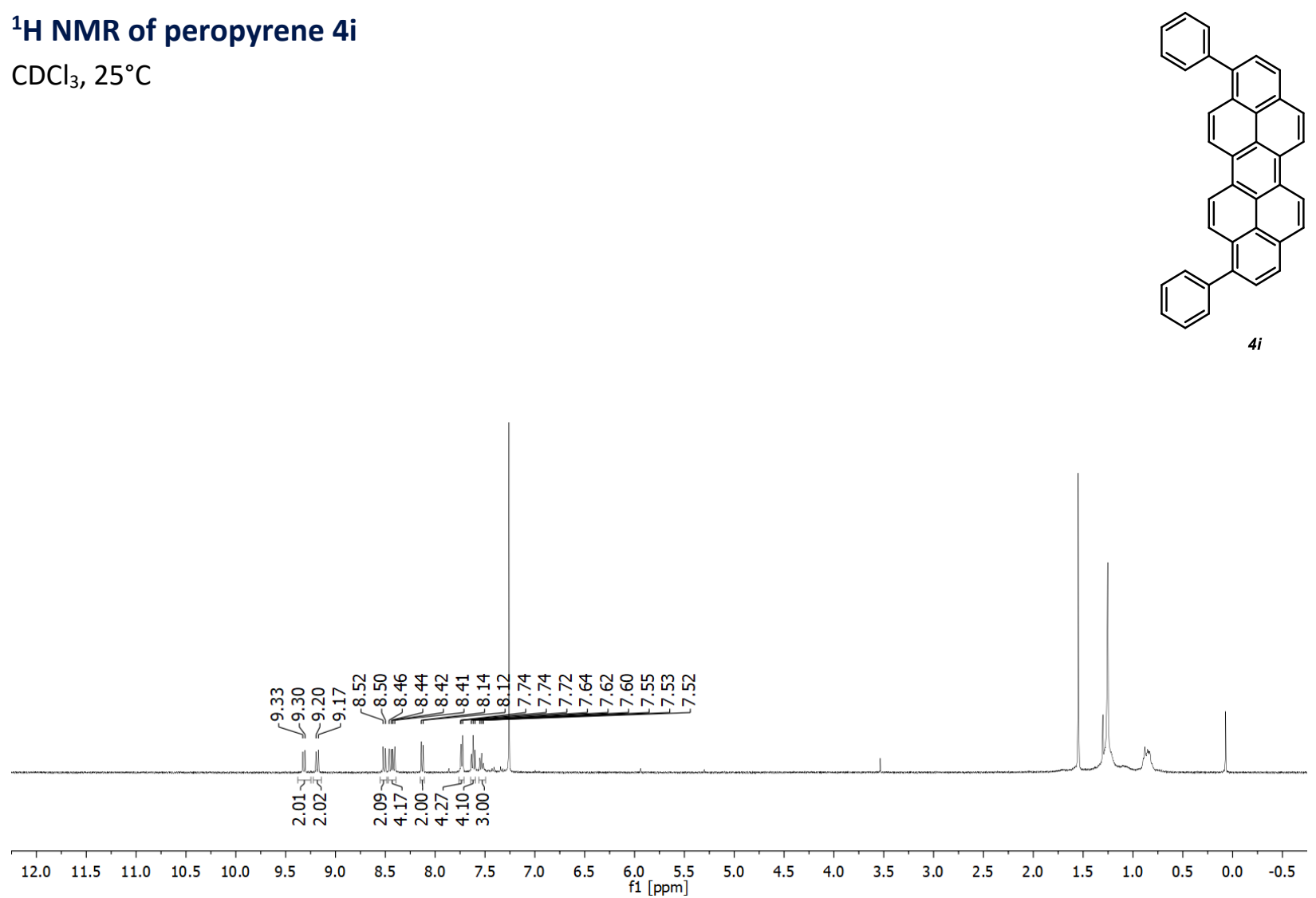

${ }^{13} \mathrm{C}$ NMR of peropyrene $4 \mathrm{i}$

$\mathrm{CDCl}_{3}, 25^{\circ} \mathrm{C}$

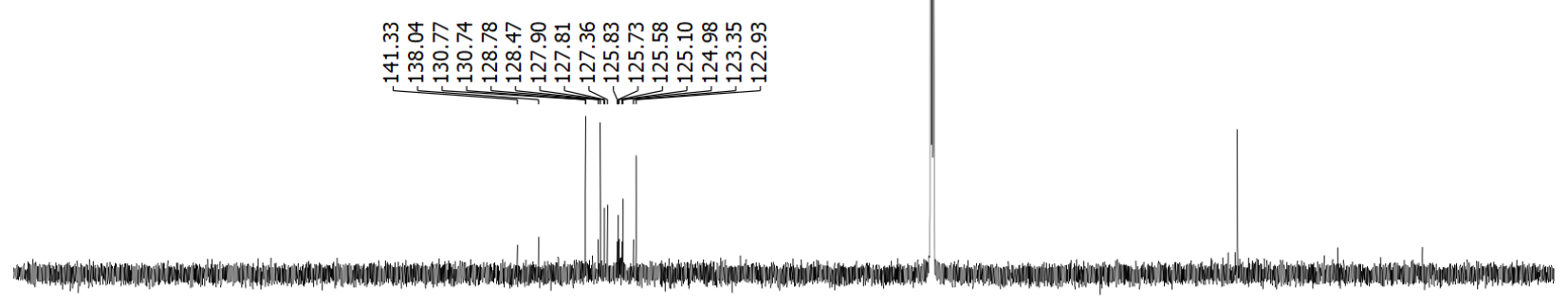

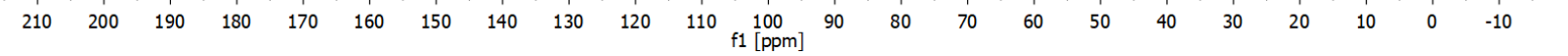


${ }^{1} \mathrm{H}$ NMR of product 9

$\mathrm{CDCl}_{3}, 25^{\circ} \mathrm{C}$
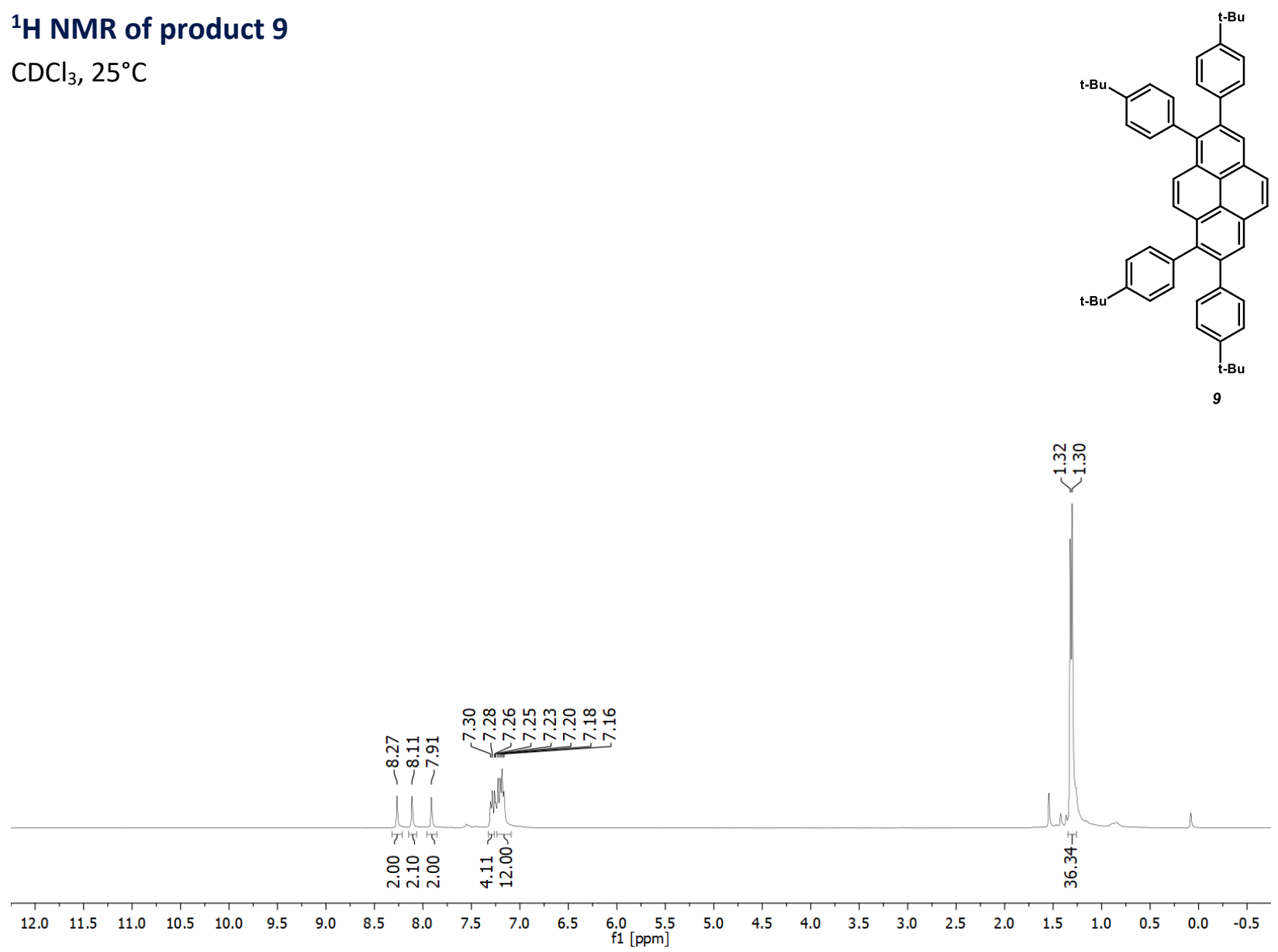

\section{${ }^{13} \mathrm{C}$ NMR of product 9}

$\mathrm{CDCl}_{3}, 25^{\circ} \mathrm{C}$

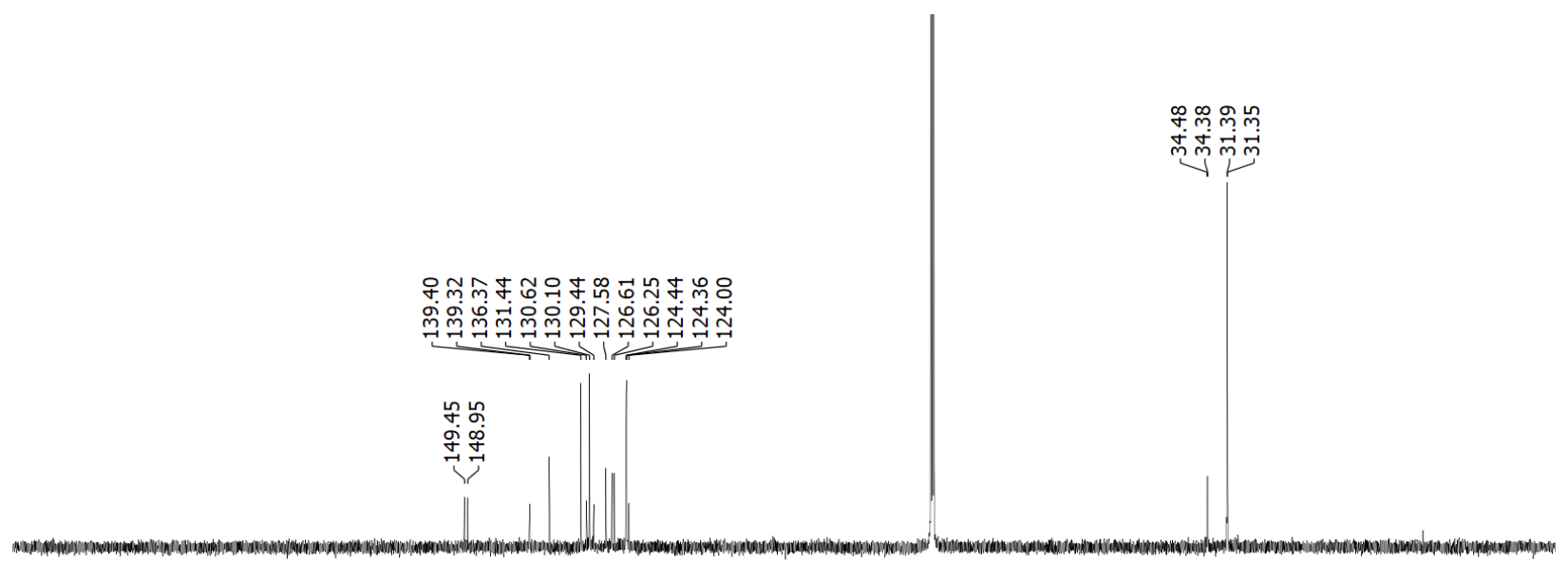

$\begin{array}{lllllllllllllllllllllll}210 & 200 & 190 & 180 & 170 & 160 & 150 & 140 & 130 & 120 & 110 & 100 & 90 & 80 & 70 & 60 & 50 & 40 & 30 & 20 & 10 & 0 & -10\end{array}$ 
${ }^{1} \mathrm{H}$ NMR of product 10

$\mathrm{CDCl}_{3}, 25^{\circ} \mathrm{C}$

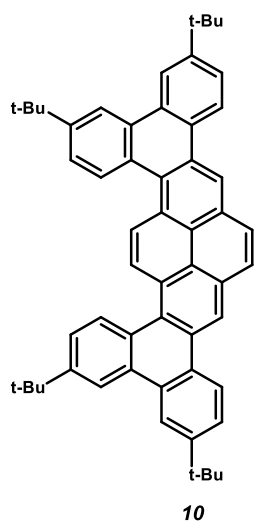

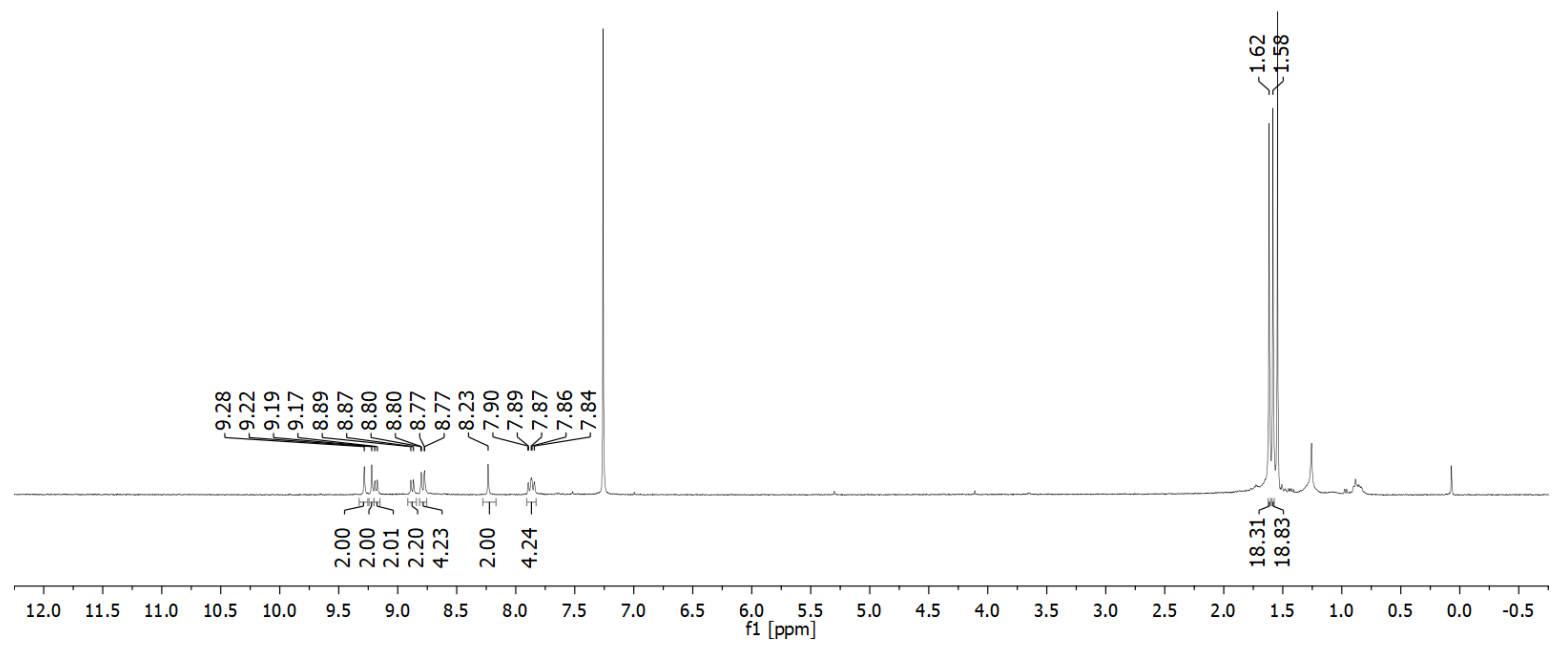

${ }^{13} \mathrm{C}$ NMR of product 10

$\mathrm{CDCl}_{3}, 25^{\circ} \mathrm{C}$

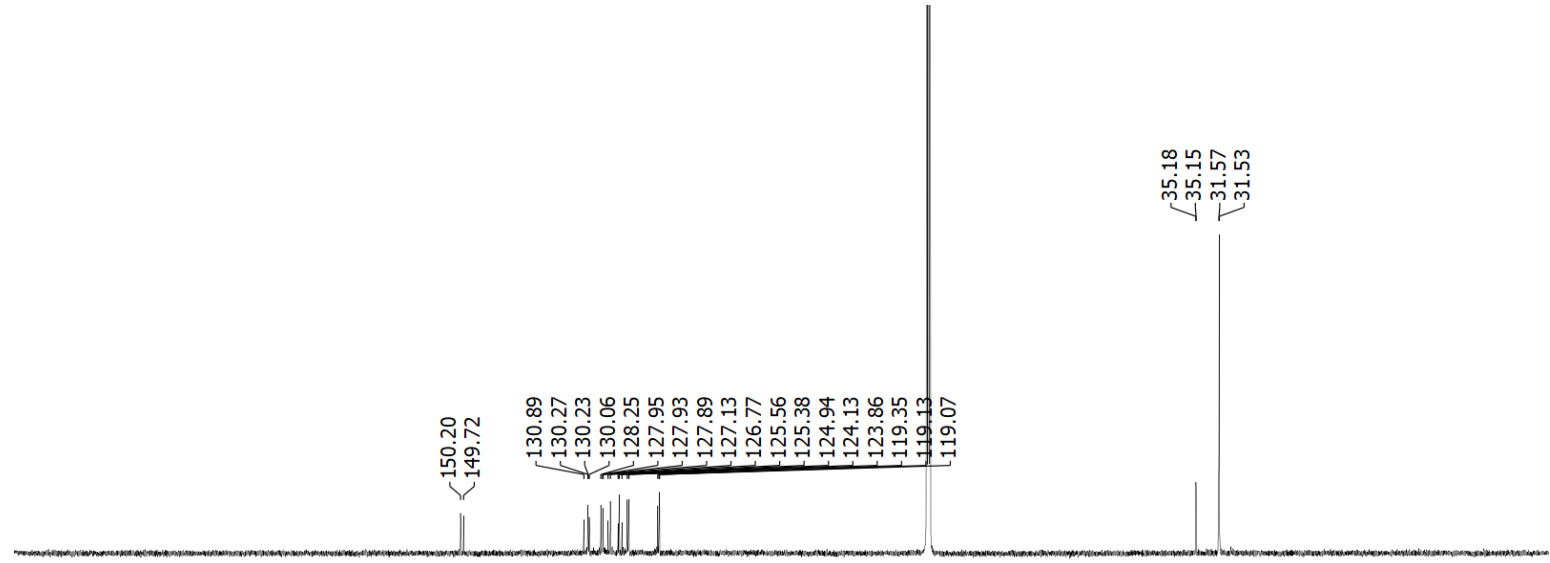

$\begin{array}{lllllllllllllllllllllllll}20 & 210 & 200 & 190 & 180 & 170 & 160 & 150 & 140 & 130 & 120 & 110 & \begin{array}{c}100 \\ \mathrm{f} 1[\mathrm{ppm}]\end{array} & 90 & 80 & 70 & 60 & 50 & 40 & 30 & 20 & 10 & 0 & -10 & -2\end{array}$ 


\section{REFERENCES}

(1) Lohr, A.; Swager, T. M. Stabilization of the Nematic Mesophase by a Homogeneously Dissolved Conjugated Polymer. J. Mater. Chem. 2010, 20 (37), 8107. https://doi.org/10.1039/c0jm00621a.

(2) Achary, B. S.; Gokulnath, S.; Ghosh, S.; Mrinalini, M.; Prasanthkumar, S.; Giribabu, L. Unprecedented Charge-Transfer Complex of Fused Diporphyrin as Near-Infrared Absorption-Induced High-Aspect-Ratio Nanorods. Chem. - An Asian J. 2016, 11 (24), 34983502. https://doi.org/10.1002/asia.201601363.

(3) de Mello, J. C.; Wittmann, H. F.; Friend, R. H. An Improved Experimental Determination of External Photoluminescence Quantum Efficiency. Adv. Mater. 1997, 9 (3), 230-232. https://doi.org/10.1002/adma.19970090308.

(4) M. J. Frisch, G.; Trucks, W.; Schlegel, H. B. .; Scuseria, G. E. .; Robb, M. A. .; Cheeseman, J. R.; Scalmani, G.; Barone, V. .; Mennucci, B. .; Petersson, G. A.; et al. Gaussian 09, Gaussian, Inc., Wallingford CT; 2009.

(5) Zhao, Y.; Truhlar, D. G. The M06 Suite of Density Functionals for Main Group Thermochemistry, Thermochemical Kinetics, Noncovalent Interactions, Excited States, and Transition Elements: Two New Functionals and Systematic Testing of Four M06-Class Functionals and 12 Other Functionals. Theor. Chem. Acc. 2008, 120 (1-3), 215-241. https://doi.org/10.1007/s00214-007-0310-x.

(6) Zhao, Y.; Truhlar, D. G. Density Functionals with Broad Applicability in Chemistry. Acc. Chem. Res. 2008, 41 (2), 157-167. https://doi.org/10.1021/ar700111a.

(7) Weinhold, F.; Landis, C. R.; Glendening, E. D. What Is NBO Analysis and How Is It Useful? International Reviews in Physical Chemistry. Taylor and Francis Ltd. July 2, 2016, pp 399440. https://doi.org/10.1080/0144235X.2016.1192262.

(8) Reed, A. E.; Weinhold, F. Natural Localized Molecular Orbitals. J. Chem. Phys. 1985, 83 (4), 1736-1740. https://doi.org/10.1063/1.449360.

(9) Reed, A. E.; Weinhold, F. Natural Bond Orbital Analysis of Internal Rotation Barriers and Related Phenomena. Isr. J. Chem. 1991, 31 (4), 277-285.

https://doi.org/10.1002/ijch.199100032.

(10) Reed, A. E.; Curtiss, L. A.; Weinhold, F. Intermolecular Interactions from a Natural Bond Orbital, Donor-Acceptor Viewpoint. Chem. Rev. 1988, 88 (6), 899-926. https://doi.org/10.1021/cr00088a005.

(11) Weinhold, F. Natural Bond Orbital Methods in: P.v.R. Schleyer. In Encyclopedia of Computational Chemistry; 1998; pp 1793-1810.

(12) Grimme, S.; Bannwarth, C.; Shushkov, P. A Robust and Accurate Tight-Binding Quantum Chemical Method for Structures, Vibrational Frequencies, and Noncovalent Interactions of Large Molecular Systems Parametrized for All Spd-Block Elements $(Z=1-86)$. J. Chem. Theory Comput. 2017, 13 (5), 1989-2009. https://doi.org/10.1021/acs.jctc.7b00118.

(13) Grimme, S. Exploration of Chemical Compound, Conformer, and Reaction Space with MetaDynamics Simulations Based on Tight-Binding Quantum Chemical Calculations. J. Chem. Theory Comput. 2019, 15 (5), 2847-2862. https://doi.org/10.1021/acs.jctc.9b00143.

(14) Szczepanik, D. W.; Andrzejak, M.; Dominikowska, J.; Pawełek, B.; Krygowski, T. M.; Szatylowicz, H.; Solà, M. The Electron Density of Delocalized Bonds (EDDB) Applied for Quantifying Aromaticity. Phys. Chem. Chem. Phys. 2017, 19 (42), 28970-28981. https://doi.org/10.1039/c7cp06114e.

(15) Legault, C. Y. CYLview, 1.0b. Univ. Sherbrooke 2009.

(16) Pettersen, E. F.; Goddard, T. D.; Huang, C. C.; Couch, G. S.; Greenblatt, D. M.; Meng, E. C.; 
Ferrin, T. E. UCSF Chimera-A Visualization System for Exploratory Research and Analysis. J. Comput. Chem. 2004, 25 (13), 1605-1612. https://doi.org/10.1002/jcc.20084.

(17) ChemCraft 1.7, Build Number 405; Http://Www.Chemcraftprog. Com (Accessed February 2015).

(18) Rodríguez-Guerra Pedregal, J.; Gómez-Orellana, P.; Maréchal, J. D. ESIgen: Electronic Supporting Information Generator for Computational Chemistry Publications. J. Chem. Inf. Model. 2018. https://doi.org/10.1021/acs.jcim.7b00714. 